\title{
Land-use change and rural development in Indonesia: Economic, institutional and demographic aspects of deforestation and oil palm expansion
}

\author{
Dissertation \\ To obtain the Ph.D. degree \\ in the International Ph.D. Program for Agricultural Sciences in Göttingen \\ (IPAG) \\ at the Faculty of Agricultural Sciences, \\ Georg-August-University Göttingen, Germany
}

Presented by

Christoph Alexander Kubitza

born in Sandton (South Africa)

Göttingen, March 2018 
Name of supervisor: Prof. Dr. Matin Qaim

Name of co-supervisor: Prof. Dr. Bernhard Brümmer

Member of Examination Committee: Prof. Dr. Krisztina Kis-Katos

Date of dissertation: 18.05 .2018 


\section{Summary}

Changes in land use, such as deforestation, expansion of sedentary agriculture and intensification of agricultural systems, significantly altered economic and ecological conditions in many regions of the world. In recent decades, one of the most rapid changes in land use was the expansion of plantation crops and the associated loss of tropical rainforests. In particular, oil palm expanded rapidly due to the increasing global demand for vegetable oil and the high land productivity of oil palm compared to other oil crops. One of the countries where the expansion of oil palm has been particularly strong during the last 25 years is Indonesia. The oil palm acreage in Indonesia increased from about 1 million hectares in 1990 to 12 million hectares in 2016. A number of studies have shown that the oil palm expansion led to increasing welfare of smallholder farmers. However, oil palm expansion was also found to aggravate ecological hazards such as greenhouse gas emission and biodiversity loss, not only by replacing the natural ecosystem functions of forests but also by replacing less-intensive agricultural production systems (e.g., rubber agro-forestry). The positive economic effects in the smallholder sector and the negative ecological effects depict a strong trade-off for policy-makers. In order to mitigate such trade-offs and ensure economic and ecological sustainability of agrarian systems, detailed studies of the impacts and determinants of land-use change are indispensable. The dissertation contains three essays on the impacts and determinants of land-use changes in Indonesia. The first essay explores the pathways through which secure property rights curb deforestation via land-sparing intensification. The second essay discusses the effect of oil palm cultivation on smallholder farmers' welfare. The last essay investigates the impact of oil palm expansion on human population growth assessed in terms of women's fertility rates (number of live birth per woman) channeled through income gains, rising returns to education, and other mechanisms.

The expansion of agricultural land remains one of the main drivers of deforestation in tropical regions, with severe negative environmental consequences. The first essay hypothesizes that stronger land property rights could enable farmers to increase input-use intensity and productivity on the already cultivated land, thus reducing incentives to expand their farms by deforesting additional land. The current literature on the land property rights and deforestation analyzed primarily the effects of secure forest property 
rights on protecting forest from encroachment. For agricultural land, studies have focused on the effects of secure property rights on input intensity and crop productivity. Examining the potential effects of secure property rights for agricultural land on deforestation via agricultural intensification was rarely explored in the literature. To test our hypothesis, we compiled a data set using various kinds of data, including a panel survey of farm households in Jambi, Sumatra, satellite imageries from LANDSAT to account for spatial patterns, such as historical forest locations and data on topsoil characteristics of farmers' plots. Results show that plots for which farmers hold formal land titles are cultivated more intensively and are more productive than untitled plots, even after controlling for other relevant factors such as soil characteristics. However, our results also show that, due to land policy restrictions, farmers located at the historic forest margins are less likely to hold formal titles for the land they cultivate. We assume that without land titles, these farmers are less able to intensify and more likely to expand into the surrounding forest land to increase agricultural output. Indeed, historic forest closeness and past deforestation activities by households are found to be positively associated with current farm size. The findings suggest that unregulated deforestation activities of farmers in combination with insecure property rights for the appropriated land are not conducive for forest conservation. Farmers with insecure property rights face incentives for extensive rather than intensive production systems, which could lead to even further deforestation if land and forest governance is weak.

While the negative ecological effects of the rapid expansion of oil palm in Southeast Asia are far-reaching and relatively widely studied, the socioeconomic consequences have received much less attention in the literature. The second essay examines the welfare effects of oil palm cultivation for smallholder farm households. Unlike other related studies that have used cross-section data, our analysis builds on panel data. Farm household data were collected from 683 farm households in Jambi, Sumatra, in two survey rounds, 2012 and 2015. The results show that oil palm cultivation has significant positive effects on farmers' consumption expenditure, our proxy of household living standards. Lower labor requirements allow oil palm farmers to further expand their farmland or reallocate the saved labor to non-farm economic activities, thus contributing to additional secondary income gains. We further test if oil palm cultivation leads to spillover effects on neighboring farm households. We find no such spillover effects, suggesting that the overall 
effect for the farming community is positive. The results further show that the positive welfare effect depends on the relative price of palm oil compared to rubber, the main competing crop in the region. Our results suggest that policies aimed at regulating further oil palm expansion will have to account for the economic benefits that this crop offers to the local population.

While we provided evidence of positive income (consumption expenditure) effects of oil palm for cultivating farmers, we expect that the ramifications of the rapid expansion of oil palm may be more far-reaching, potentially also affecting some of the underlying determinants of economic development, such as population growth. The proliferation of new production technologies is often regarded as one of the key drivers of the historical fertility transition in the US and Western Europe. In contrast, empirical evidence on the relationship between technologies, including crop choice, and fertility in developing countries such as Indonesia is largely inexistent. The third essay of this dissertation addresses this research gap, exploring the effect of oil palm expansion on fertility in Indonesia using a range of different data sources at the regency level. Oil palm is less labor-intensive than some of the alternative crops such as rubber or rice. Hence, in a landscarce setting, the substitution of oil palm for other crops induces labor savings similar to mechanization. We use Becker's quantity-quality model to identify different causal mechanism through which the expansion of oil palm could affect the number of children born to a woman (fertility). Our identification strategy relies on an instrumental variable approach with regency-fixed effects, in which the expansion of area under oil palm at regency level is instrumented by regency-level attainable yield of oil palm interacted with the national oil palm expansion. While a labor-saving technology could theoretically increase fertility rates by decreasing maternal opportunity costs of time, we find consistently negative effects of the oil palm expansion on fertility. The results suggest that income gains among agricultural households coupled with broader local economic development explain this effect. Specifically, local economic development seems to have raised returns to education and triggered investments into women's and children's education, which together with direct income effects explain the bulk of the negative effect of the oil palm expansion on fertility. 
Overall, our findings are in line with previous studies, suggesting that smallholderdriven oil palm expansion has on average positive socioeconomic effects. However, the negative ecological effects are also widely documented. Our research underlines that having secure and clear property rights for agricultural land and forest as well as access to the non-agricultural sector might be important steps towards more sustainable land-use systems in Indonesia. 


\section{Acknowledgements}

I thank my supervisor, Prof. Dr. Matin Qaim, for his guidance and helpful feedback while writing my thesis and for encouraging me to explore a diverse set of research questions during my $\mathrm{PhD}$. I also thank Prof. Dr. Bernhard Brümmer and Prof. Krisztina Kis-Katos for being members of my thesis committee.

I also want to thank Vijesh Krishna for his continuing support and advice during the countless coffees in Göttingen and our field research in Indonesia. I am sure café z will miss us. I am also thankful for the thorough advice and feedback of Esther Gehrke during my PhD.

Our survey in Indonesia would not have been possible without our field work team, including Datitta, Edo, Fauzan, Juwita, Khotimah, Niko, Rico, Tito, Tuti, Wila, Winda and Jean. Makasih! I would also like to extend my gratitude to our CRC (Collaborative Research Center) C07 counterparts Prof. Hermanto Siregar from Bogor Agricultural University and Prof. Zulkifli Alamsyah from Jambi University for supporting our research in Indonesia.

I am grateful for the support of my CRC colleagues in Bogor, Jambi and Göttingen, also for sharing their data with me - special thanks to the B05 and C01 team. And I want to thank my Chair colleagues Kibrom, Jonida, Zewdu, Daniel, Rosina, Kenneth and Theda for sharing coffees and ideas.

Vielen Dank auch an meine Familie für ihre Unterstützung in den letzten drei Jahren. Auch meinen Freunden hier in und um Göttingen danke ich - ohne euch wäre es eine andere Zeit gewesen. Ein besonderer Dank geht natürlich an meine Freundin Svenja für ihre Ausdauer und Unterstützung, die uns sicher bis ans Ende getragen hat.

This research was financially supported by Deutsche Forschungsgemeinschaft (DFG) as part of the Collaborative Research Center (CRC 990): Ecological and Socioeconomic Functions of Tropical Lowland Rainforest Transformation Systems (Sumatra, Indonesia). 


\section{Table of contents}

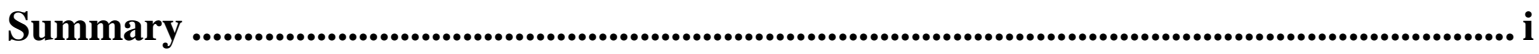

Acknowledgements ...................................................................................................................... $\mathrm{v}$

List of tables ............................................................................................................................. ix

List of figures .............................................................................................................................. $\mathrm{xi}$

1 General introduction ................................................................................................ 1

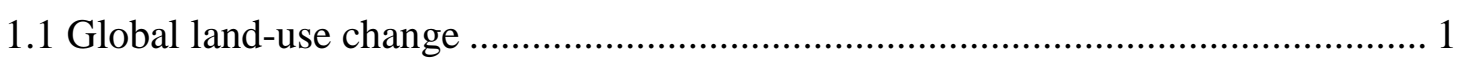

1.2 Land-use change in Indonesia .......................................................................... 3

1.3 Research objectives .................................................................................... 5

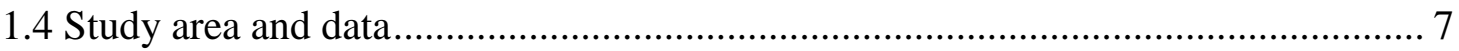

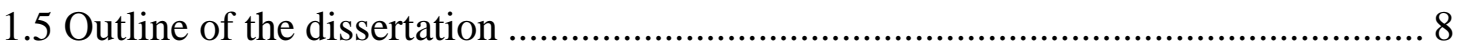

2 Land property rights, agricultural intensification, and deforestation in Indonesia 10

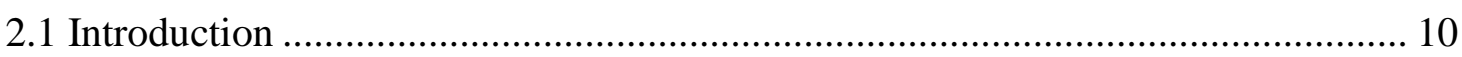

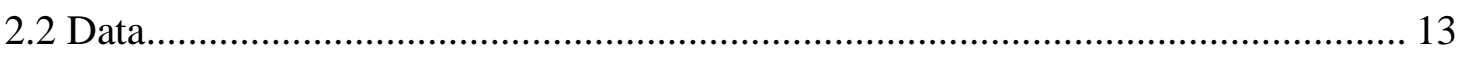

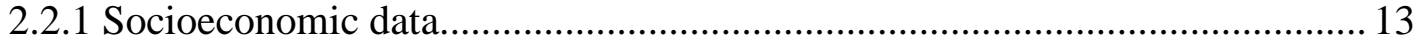

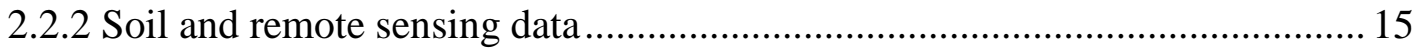

2.3 Econometric methods ................................................................................. 15

2.3.1 Models to analyze agricultural productivity.................................................... 16

2.3.2 Models to analyze agricultural intensity ....................................................... 18

2.3.3 Spatial regression models......................................................................... 18

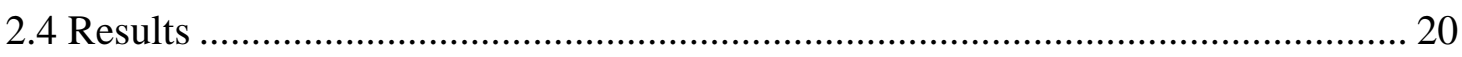

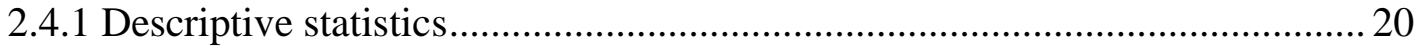

2.4.2 Land titles and agricultural productivity ..................................................... 22

2.4.3 Land titles and agricultural intensity .......................................................... 24

2.4.4 Spatial patterns of land titling ...................................................................... 24

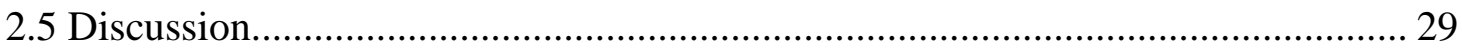

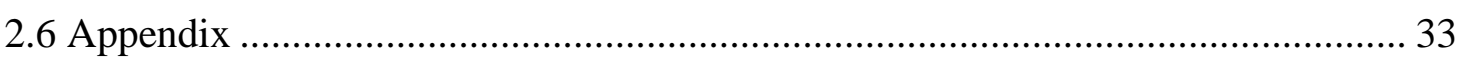

3 The economics behind an ecological crisis: Livelihood effects of oil palm expansion in Sumatra, Indonesia ......................................................................................................... 41

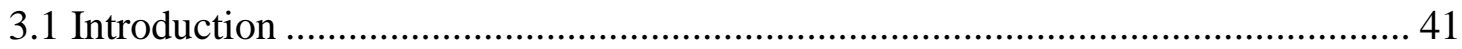

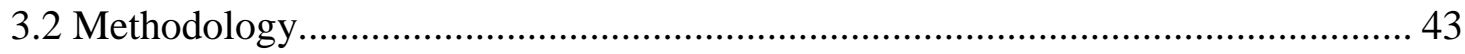

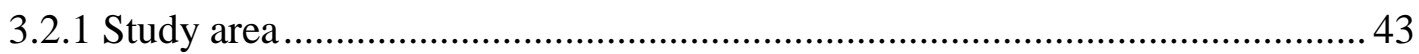


3.2.3 Measuring livelihoods

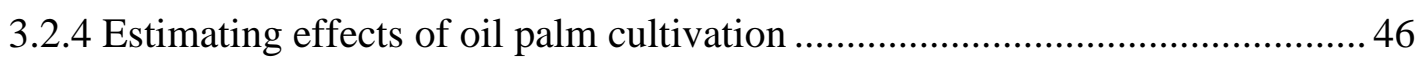

3.2.5 Estimating temporal differences in oil palm effects................................... 47

3.2.6 Estimating spatial patterns and spillovers ............................................... 47

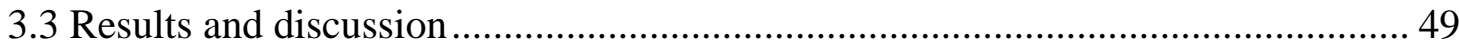

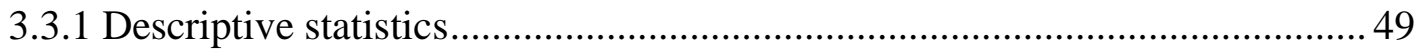

3.3.2 Average livelihood effects of oil palm cultivation ...................................... 52

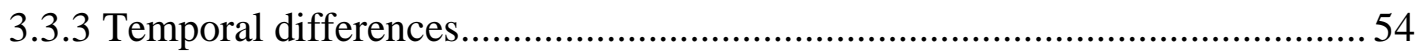

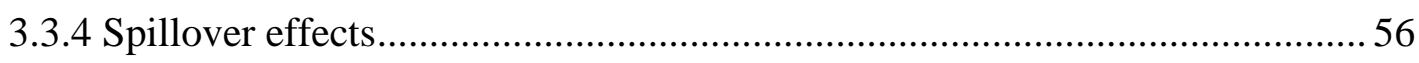

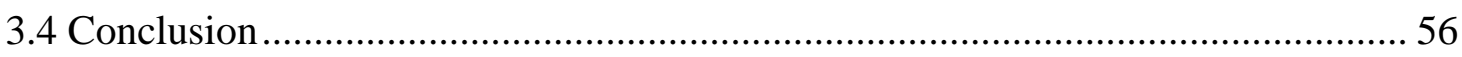

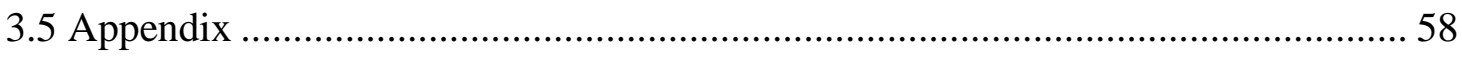

4 Why does a labor-saving technology decrease fertility rates? Evidence from the oil palm boom in Indonesia .................................................................................................................. 62

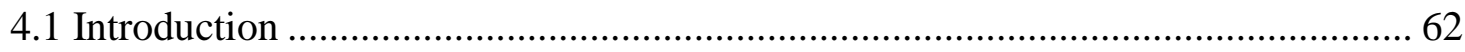

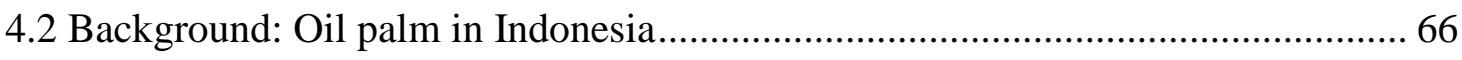

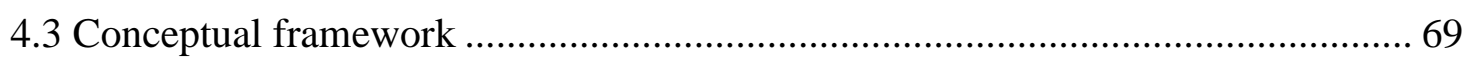

4.3.1 A simple model on oil palm expansion and demand for children.................. 69

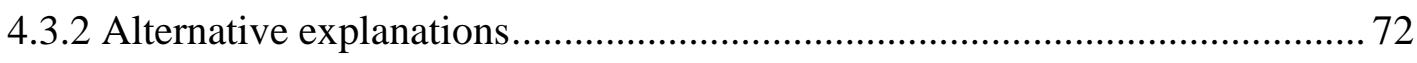

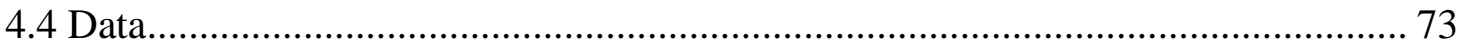

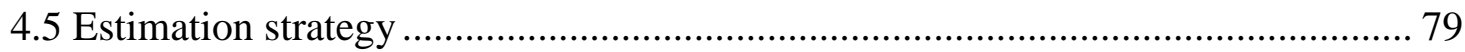

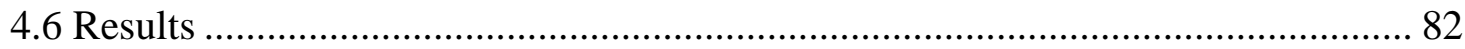

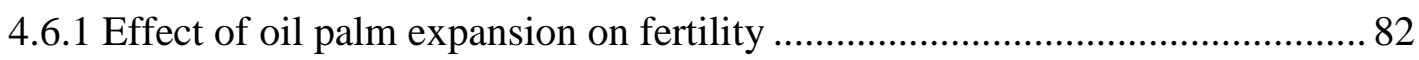

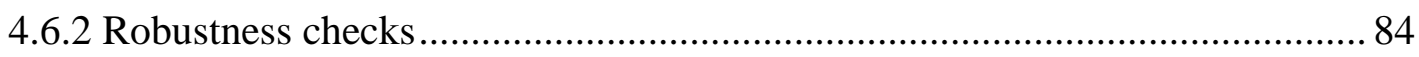

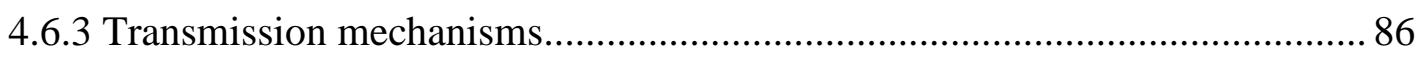

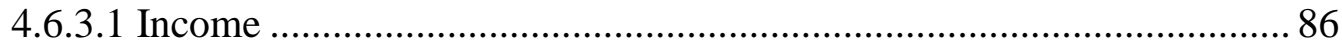

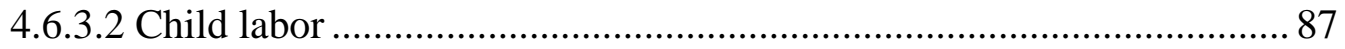

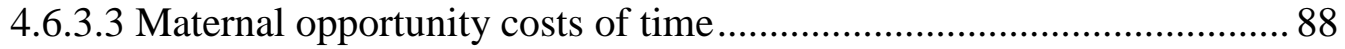

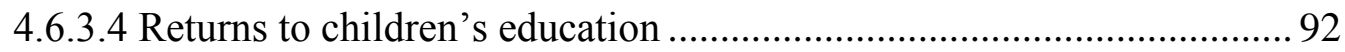

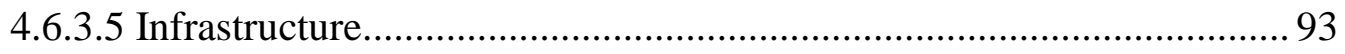

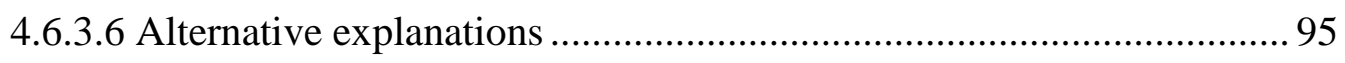

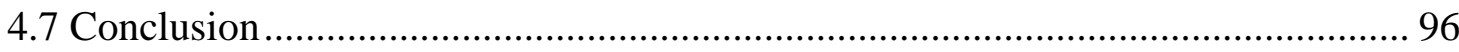




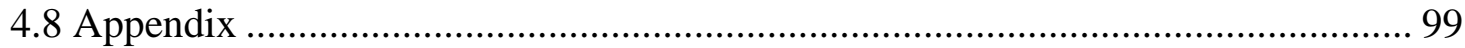

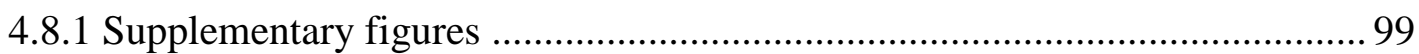

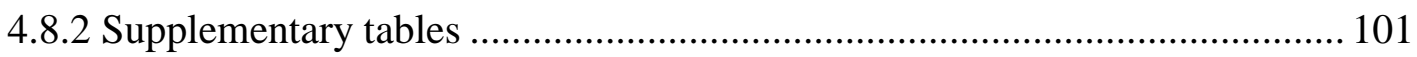

5 General conclusion .......................................................................................... 110

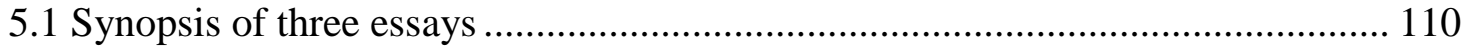

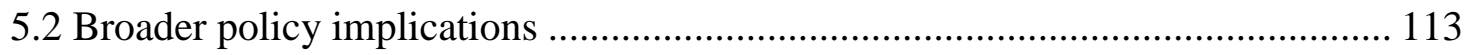

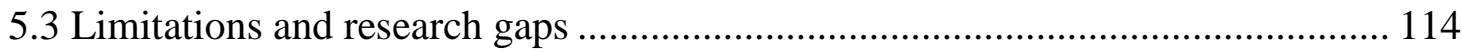

References........................................................................Fehler! Textmarke nicht definiert.

General appendix.................................................................................................................................. 127

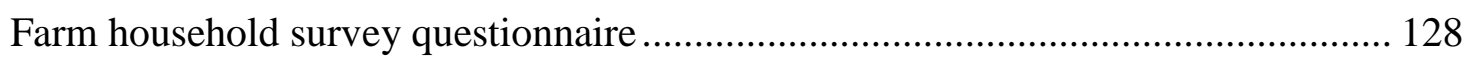




\section{List of tables}

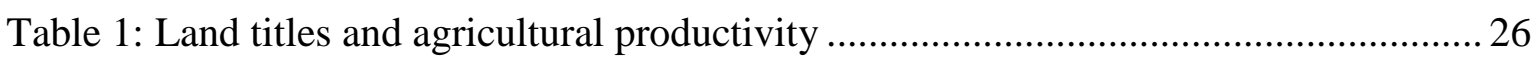

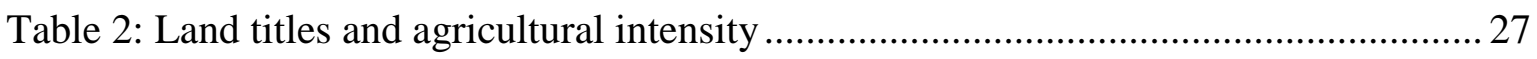

Table 3: Historical forest coverage, land titles, and farm size ........................................ 28

Table 4: Differences between oil palm cultivators and non-cultivators ............................. 50

Table 5: Returns to labor and land for oil palm and rubber ............................................ 51

Table 6: Labor and land productivity of oil palm and rubber ........................................... 67

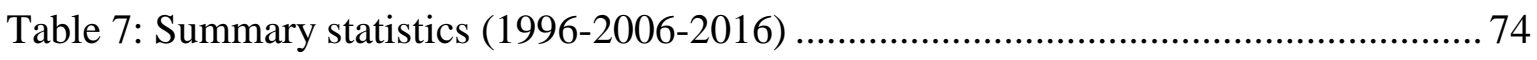

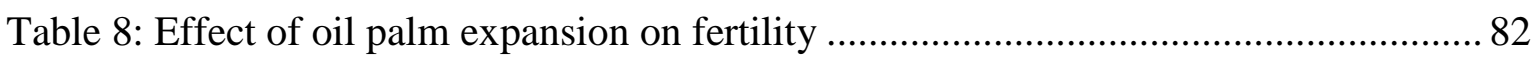

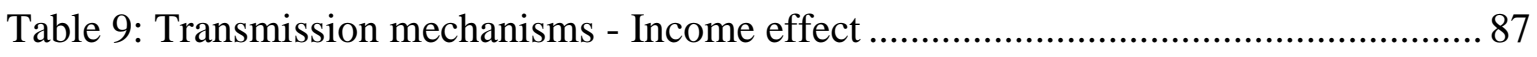

Table 10: Transmission mechanisms - Opportunity costs of time …................................... 89

Table 11: Transmission mechanisms - Opportunity costs of time II....................................90

Table 12: Effect of oil palm on wages - Returns to education ......................................... 91

Table 13: Transmission mechanisms - Returns to children's education .............................93

Table 14: Transmission mechanisms - Infrastructure......................................................... 94

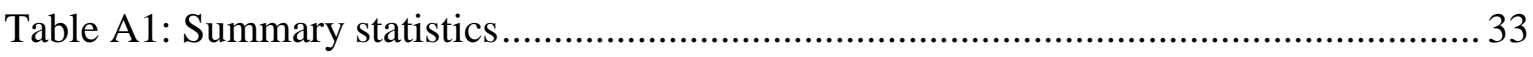

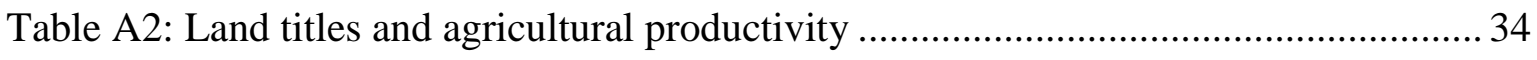

Table A3: Historical forest coverage and land titles (spatial error and spatial lag models) 36

Table A4: Historical forest coverage and farm size (spatial error and OLS models) ......... 37

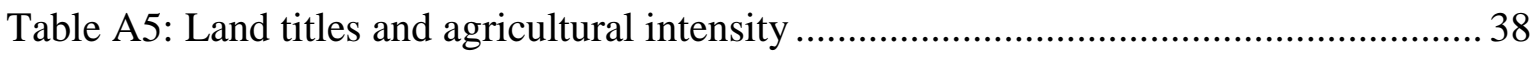

Table A6: Historical forest coverage, land titles, and farm size........................................ 39

Table A7: Agricultural productivity and farm size ....................................................... 40

Table A8: Differences in input use between rubber and oil palm cultivation in 2012 and

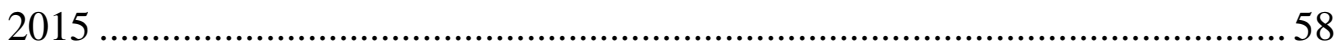

Table A9: Impact of oil palm cultivation on total consumption expenditures ....................59

Table A10: Impact of oil palm cultivation on non-food consumption expenditures........... 60

Table A11: Impact of oil palm cultivation on food consumption expenditures ................... 61

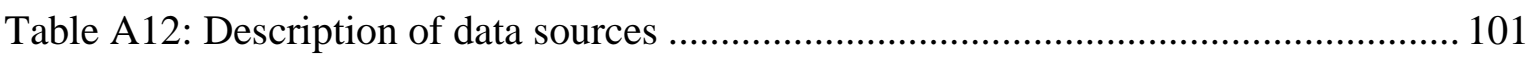

Table A13: Effect of oil palm expansion on fertility - 1993-2003 ................................. 102

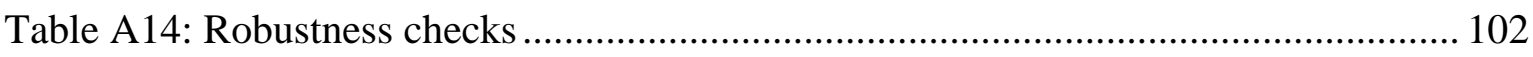


Table A15: Effect of oil palm expansion on fertility - Five year differences 102

Table A16: Transmission mechanisms - Child labor....................................................... 103

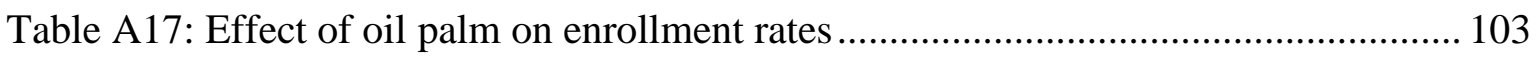

Table A18: Effect of oil palm on women's non-agricultural wages ................................. 104

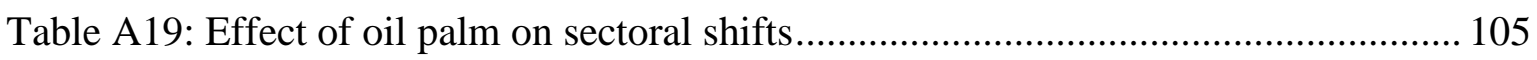

Table A20: Effect of oil palm on wages - Returns to education ...................................... 106

Table A21: Transmission mechanisms - Returns to children's education II..................... 106

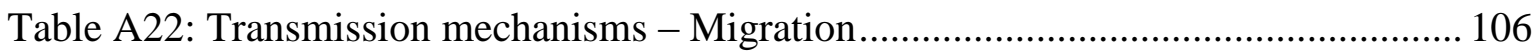

Table A23: Transmission mechanisms - Child mortality …........................................... 107

Table A24: Transmission mechanisms - Female empowerment .................................... 107

Table A25: Transmission mechanisms - Female empowerment II ................................. 108

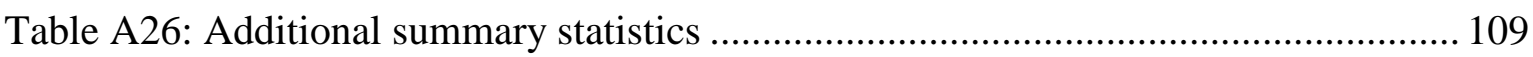




\section{List of figures}

Figure 1: Maps of land uses in Jambi Province (Sumatra) in 1990 and 2013 .................... 21

Figure 2: Land titling and deforestation activities by farmers (1965-2015)....................... 22

Figure 3: Map of Jambi Province showing sample villages ............................................. 45

Figure 4: Price movement of rubber and palm between 2012 and 2016 ............................ 52

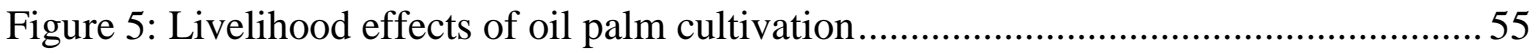

Figure 6: Regional oil palm expansion of smallholders in Indonesia in 1996 and 2015 .... 75

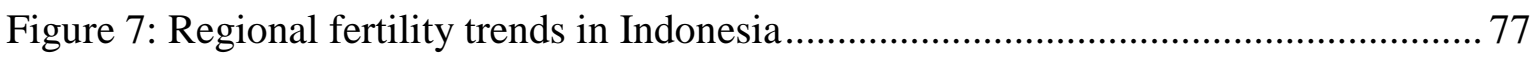

Figure 8: Regency-wise attainable yield for oil palm in Indonesia .................................. 79

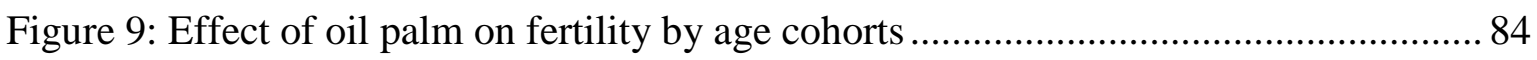

Figure A1: Expansion of oil palm in Indonesia by producer type ................................. 99

Figure A2: Expansion of plantation crops in Indonesia ................................................... 99

Figure A3: Effect of oil palm expansion on fertility using lags ...................................... 100

Figure A4: Effect of oil palm expansion on fertility using DHS data .............................. 100 


\section{General introduction}

\subsection{Global land-use change}

For millennia, human activities have changed land use throughout the world driven primarily by an ever increasing demand for agricultural products (Foley et al. 2005; Lambin \& Meyfroidt 2011; van Vliet et al. 2015). Population growth, rising incomes and globally changing preferences are the main drivers of agricultural demand, and projections of future trends do not yet indicate any turning point (Alexandratos \& Bruinsma 2012; Schmitz et al. 2014). Global population is expected to grow to over nine billion by 2050 (UNDP 2015). The expansion of urban settlements may appropriate an additional 30 million hectares of fertile cropland by 2030. This lost cropland will have to be replaced by converting natural habitat into agricultural land unless sufficient intensification and productivity growth occurs to make up for declining areas (Bren d'Amour et al. 2016). Forecasts on economic growth suggest that per-capita income will rise throughout the world, particularly in low-income countries (OECD-FAO 2016). Such income growth will help to decrease food insecurity, but will also increase the demand for agricultural products per capita. Income growth and urbanization are also expected to change dietary habits. In particular the shift from plant-based staple foods to meat and dairy products will require more land per calorie (Pingali 2007; Qaim 2017).

More sustainable consumption patterns - with less waste and lower consumption of animal-source foods - can influence future demand to some extent, but will not suffice to completely reverse the trend. Rising demand for food and other agricultural products is hence likely to require cropland expansion, or strong productivity growth ${ }^{1}$ on the already cultivated land, or a combination of both. In the past, cropland expansion mainly occurred through the conversion of natural habitats such as forests. While deforestation rates stabilized or even reversed in temperate and subtropical regions, this is not the case in tropical zones. More than $83 \%$ of new agricultural land was appropriated from forests between 1980 and 2000 in these zones (Gibbs et al. 2010). In total between 1990 and 2015, six to ten million hectares of forest were lost annually (FAO 2016). However, although

\footnotetext{
${ }^{1}$ In principle, growth in land productivity can occur either through agricultural intensification (more inputs per unit of land) or through technological change (growth in total factor productivity).
} 
agricultural production rose by more than $200 \%$ between 1955 and 2005, total global cropland increased by only $15 \%$ (Schmitz et al. 2014). These numbers show that productivity growth on the already cultivated land played a significant role in terms of reducing the pressure on land, using the toolbox of modern agricultural technologies.

While catering for the needs of a growing population, both productivity growth and agricultural expansion involve certain trade-offs concerning the ecosystem functions of land. Ecosystem functions are here understood as 'the capacity of natural processes and components to provide goods and services that satisfy human needs, directly or indirectly' (Groot 1992). Converting forest to agricultural land adversely affects regulation functions such as carbon storage but also soil fertility and water regulation and supply. Moreover, habitat functions such as species diversity and density and information functions such as recreational and cultural components can be affected (Fearnside 2005; Foley et al. 2005; Butler \& Laurance 2009; Wilcove et al. 2013; Barnes et al. 2014; Dislich et al. 2017). Increasing productivity and intensity within agricultural systems can also affect ecosystem functions such as on-farm biodiversity, soil fertility and water regulation (Tscharntke et al. 2012). On the other hand, maintaining and increasing production functions such as the supply of agricultural goods are indispensable. Due to the existence of numerous such trade-offs, analyzing the impacts of different land uses on ecosystem functions has become a major focus of many research projects (Tilman et al. 2002; Steffan-Dewenter et al. 2007; Clough et al. 2016).

This dissertation is written as part of such a research project which addresses these issue in the context of Indonesia - the Collaborative Research Center (CRC) 990 "Ecological and Socioeconomic Functions of Tropical Lowland Rainforest Transformation Systems" financed by the "Deutsche Forschungsgemeinschaft" (DFG). The CRC 990 aims to provide evidence on how to protect ecosystem functions of forest and agricultural landuse systems, while enhancing economic welfare. This includes reconciling agricultural production with ecosystem functions such as biodiversity, developing new ways of sustainable agricultural land use. The CRC research aims to enable policy-makers to select the most efficient allocation and types of land uses based on the preferred set of ecosystem functions within their society. Besides identifying the impact of different land uses on ecosystem functions, another strand of research examines the institutional and socioeconomic factors that determine land-use change (Euler et al. 2016b; Krishna et al. 
2017b), giving policy-makers a toolset how to effectively influence land use. This dissertation contributes in these directions, including three essays that examine land-use change in Indonesia. The first essay focuses on the institutional determinants of land-use change, while the second and third essays focus on the socioeconomic impact of land-use change.

\subsection{Land-use change in Indonesia}

Indonesia depicts a hotspot of global land-use change. In particular, forest loss and the expansion of oil palm (Elaeis guineensis) occurred at rates rarely observed in the recent history of agriculture. The global production of palm oil rose by around $600 \%$ between 1990 and 2016 (Byerlee et al. 2017; US Department of Agriculture 2017), and this production growth was highly concentrated in only two countries - Indonesia and Malaysia. In Indonesia, the main producer since about 2009, oil palm covered 12 million hectares of land in 2016, rising from 1 million hectares in 1990 (Ministry of Agriculture 2017). Several factors led to this rapid increase: First, consumption of vegetable oils increased by approximately 5\% annually between 1993 and 2012, and also non-food consumption of palm oil derivatives for cosmetics and soaps increased rapidly (Byerlee et al. 2017). Second, since the early 2000s, new policies in different parts of the world incentivized the use and production of biodiesel, increasing output from only less than 1 million tons in 2003 to 31 million tons in 2015 (OECD-FAO 2015). Oil palm growth was in particular strong compared to other oil-yielding crops such as soybean, rapeseed, and sunflower due to the high land productivity of oil palm. Well-managed oil palm plantations can produce three times more oil than rapeseed fields and even six times more than soybeans. The high market demand contributed to the high financial profitability of palm oil production (Clough et al. 2016; Drescher et al. 2016; Byerlee et al. 2017). Palm oil production thus became an essential part of Indonesia's past and current economic development strategy. In the past, oil palm expansion joint with resettlement programs (the so-called transmigrant program) was used to balance the divergent population density of Java and the outer islands and to foster economic development in remote regions. With financial support from the World Bank and other international institutions 3.5 million 
people were resettled by 1990 to the outer islands (Adhiati \& Bobsien 2001). Transmigrants became hence an essential part of Indonesia's palm oil sector.

The increase in palm oil production was mainly driven by expansion in planation area and not by any technological innovation. There is some evidence from Indonesia which showed that oil palm expansion led to the conversion of other agricultural land uses such as agroforestry or rubber plantations (Gatto et al. 2015). However, the increase in production volume also caused the loss of tropical rainforest and conversion of peat swamps, contributing significantly to heavy deforestation and greenhouse gas emission in Indonesia (Wilcove et al. 2013; Vijay et al. 2016). With 0.84 million hectares of forest lost in 2012, Indonesia's deforestation rate was the highest worldwide surpassing even Brazil (Margono et al. 2014). The adverse effects of deforestation on various ecosystem functions are well known. Java, Sumatra, Kalimantan and Malaysian peninsula inhabit the highest number of endemic plants worldwide compared to other biodiversity hotspots (Myers et al. 2000). Moreover, since Indonesia's tropical forests are major stocks for carbon dioxide, the deforestation is threatening global climate stability (Carlson et al. 2012). Detailed studies further document the detrimental effects on other ecosystem functions if forest is converted into monocultures (Fitzherbert et al. 2008; Barnes et al. 2014; Clough et al. 2016; Dislich et al. 2017).

The academic literature emphasizes oil palms' negative environmental effects, however, oil palm cultivation in Indonesia sustains millions of livelihoods. Smallholder farm households cultivate more than 40 percent of the oil palm area in Indonesia (Gatto et al. 2015; Euler et al. 2016b), and non-farm household in rural areas also derive substantial income from working in oil palm farms and plantations (Bou Dib et al. 2018). While the literature is rather critical on the potential welfare effects of large-scale plantations (Li 2015; Cramb \& McCarthy 2016b), recent research shows that the welfare effects of oil palm cultivation are substantial for smallholder farmers (Euler et al. 2017; Krishna et al. 2017a). Smallholder farmers are often among the poorest segments of society. Hence, measures to secure and improve smallholder livelihoods are important components of poverty reduction (Sibhatu et al. 2015). However, farmers' livelihoods are also vulnerable to changes in their environment such as global warming, soil erosion, and water depletion. Trade-offs and interactions between ecosystem functions and economic gains such as 
poverty reduction are therefore in particular strong in the context of smallholder farming. This motivates the focus of this dissertation on smallholder farming in Indonesia.

\subsection{Research objectives}

In the following, we will outline the research objectives of the three essays related to institutional and socioeconomic aspects of oil palm expansion and deforestation with a particular focus on smallholder farming.

As mentioned, smallholder farming is an important part of Indonesia's palm oil sector. To access the necessary land for plantation agriculture, smallholder farmers are often found to deforest land; thus contributing to the high deforestation rates (Krishna et al. 2017b). The policies at hand to regulate deforestation of smallholders often include costly control mechanism such as fines and embargos. Yet, in Indonesia, as well as in many other tropical countries, fines and embargoes are associated with several complications, including high costs of monitoring, low acceptance rates among smallholders, and thus low effective reduction rates in deforestation (Meyfroidt et al. 2013; Godar et al. 2014). Incentive-based approaches could potentially be more promising and also more in line with poverty reduction objectives. We propose that land property rights are fundamental to improve both local livelihoods and forest protection. The current literature on land property rights and deforestation analyzed primarily the effects of secure forest property rights on protecting forest from encroachment (Damnyag et al. 2012; Liscow 2013; Robinson et al. 2014). For agricultural land, studies have focused on the effects of secure property rights on input intensity and agricultural productivity (Goldstein \& Udry 2008; Holden et al. 2009; Deininger et al. 2011). Yet, most of these studies did not link the potential effect of more secure property rights for agricultural land to deforestation outcomes. In the first essay, we hypothesize that more secure property rights at the forest margin could increase farmers' incentive to intensify production, thus reducing the incentive to increase agricultural output through deforesting additional land. We test this hypothesis by using panel data collected from smallholder farmers at household level and plot level. Moreover, we combine these survey data with data from satellite imageries and soil samples. 
The second essay analyzes the welfare effect of oil palm cultivation for smallholder farmers. Environmental studies found that biodiversity, carbon stocks and other ecosystem functions are decreasing when moving from forest or less intensive agricultural systems to oil palm monocultures (Clough et al. 2016; Dislich et al. 2017). However, economic studies provide broad evidence of a positive welfare effect of oil palm adoption and cultivation among smallholders (Rist et al. 2010; Obidzinski et al. 2012; Krishna et al. 2017a; Euler et al. 2017). Several studies underline in particular the high labor productivity of oil palm compared to other competing crops such as rubber. These studies further suggest that the associated welfare gains are mostly generated through reallocation of labor to additional land or off-farm work (Euler et al. 2017; Krishna et al. 2017a). These findings deliver important evidence to understand the rapid oil palm adoption patterns among smallholder farmers. Furthermore, the findings hold important lessons for policy-making, because the economic gains for farmers should not be ignored. Yet, the positive welfare effect found in the literature depends on several factors such as the relative price of oil palm compared to competing crops and the assumption of zero spillover effect to other farmers. Previous studies relied on cross-sectional data, so that the role of possible price changes and other dynamic effects could not be analyzed. We address these shortcomings in the previous literature by using panel data from farm household collected in 2012 and 2015, a time period where relevant output prices fluctuated considerably. We also use spatial econometric models to control for spatial dependence and test explicitly for spillover effects.

We assume that oil palm expansion is not only affecting income growth but also some of the underlying determinants of development, such as population growth. We focus on fertility (i. e., the number of child births per women), which is one of the main drivers of population growth and a central issue for policy planning. While the proliferation of new production technologies is often regarded as one of the key drivers of the historical fertility transition in the US and Western Europe, empirical evidence on the relationship between technology and fertility in developing countries such as Indonesia is largely inexistent. In the last essay, we address this gap in a detailed analysis of the effects of oil palm expansion on fertility. We show that oil palm can be characterized as a labor saving technology, releasing large amounts of labor from agriculture in the case of land scarcity. Building upon this, we develop a theoretical model for the effect of oil palm expansion on fertility 
based on Becker's Quantity-Quality model. We highlight five main mechanisms. The first mechanism is an income effect, assuming that with rising income fertility decreases. The second and third mechanisms show that reduction in the value of child labor and increasing maternal opportunity costs of time can reduce fertility. The fourth and fifth mechanisms relate to returns to education and infrastructure development which could reduce the costs of investing in child quality such as education, leading to substitution away from child quantity to quality. We also discuss three alternative mechanisms: female empowerment, migration patterns and child mortality. To test our predictions we use a wide set of nationally representative data. Land-use change is commonly associated with significant shifts in farm income levels, consumption patterns and ecosystem functions (Lambin \& Meyfroidt 2011; Clough et al. 2016; Edwards 2017), but rarely with demographic variables.

\subsection{Study area and data}

This dissertation builds on a set of different data sources from Indonesia. Primary data were collected in Jambi Province, Sumatra. The research is focused on the lowlands of Jambi Province, which present an archetype of land-use change in Indonesia with rapid expansion of oil palm and shrinking forest areas.

To obtain data on smallholder farms we conducted an extensive survey of local farm household. The household survey data were collected in two waves, 2012 and 2015. Data in 2012 were collected by another research team (Euler et al. 2016b), while data collection in 2015 was led by the author of this dissertation himself. Sampling was based on a multistage framework. All five regencies in Jambi Province located in the tropical lowland areas - Sarolangun, Batanghari, Muaro Jambi, Tebo, and Bungo - were selected purposively. From these regencies, 40 villages in 20 districts were randomly selected. Five additional villages were selected purposively to allow for interdisciplinary overlaps with other subprojects of the CRC 990 (Drescher et al. 2016). After village selection, a complete list of all farm households was compiled in each village together with the village leaders. From these lists, 6 to 24 farm households were randomly selected. The sample size was adjusted to village population size to mitigate possible sampling bias. All households that owned any agricultural land in the last 5 years were included. In total 700 farm households were 
interviewed $^{2}$. Data were collected in 2012 and 2015 for the same farm households. Between these two rounds, the attrition rate was at $6 \%$. In total 41 households from 2012 could not be re-interviewed in 2015. Outmigration was the major reason for attrition $(56 \%$ of attrition). Other reasons included refusal to be interviewed again (24\%), death or old age $(10 \%)$.

Data were collected in the dry season in 2012 and 2015 using personal interviews with structured questionnaires (the 2015 questionnaire is included in the General Appendix of this dissertation). The survey instrument included questions at the household level such as current farm income, off-farm activities, socioeconomic characteristics of the household, household composition and food and non-food consumption expenditures. Additionally, data at the plot level were collected such as land use, material and labor input, yield, acquisition pathways and tenure status. In addition, a village survey was conducted in 2012. The survey elicited land-use changes and related institutional aspects at the village level (Gatto et al. 2015). In 2015, a smaller village survey was conducted including in particular questions on village demographics, land tenure and conflicts within the village. The village questionnaires were administered to knowledgeable persons (e.g., village head, secretary etc.) in the village.

The farm household survey data and the village survey datasets were used in all the three dissertation papers. In the first essay (Chapter 2), we combine the household survey data with remote sensing data (Melati et al. 2014) as well as measurements of topsoil characteristics (Guillaume et al. 2015). The further description of the data can be found in the respective essays themselves. The third essay (Chapter 4) builds on a large set of national data. The data sources are described in the data section and the Appendix of Chapter 4.

\subsection{Outline of the dissertation}

The remainder of this dissertation is organized as follows: Chapter 2 presents the first essay, exploring the potential effect of more secure property rights on agricultural productivity, intensity and deforestation. The second essay is presented in Chapter 3,

\footnotetext{
${ }^{2}$ Out of these 700 farmers, 17 farmers were purposively selected due to interdisciplinary overlaps. These farmers were not included in the statistical analysis.
} 
analyzing the welfare effect of oil palm cultivation for smallholder farmers. The third and final essay, examining the effect of the oil palm expansion on fertility rates using nationally representative data is presented in Chapter 4 . Chapter 5 summarizes the broader findings and policy implications. It further discusses limitations of the research and proposes some directions for future research. The questionnaire for the farm household survey in 2015 is attached in the General Appendix. 


\section{Land property rights, agricultural intensification, and deforestation in Indonesia ${ }^{3}$}

\subsection{Introduction}

Deforestation remains a widespread problem, especially in tropical regions. Between 2010 and 2015, about 6 million hectares of tropical forest were lost annually (FAO 2016), entailing severe negative consequences for biodiversity, ecological systems, and climate stability (Fearnside 2005; Butler \& Laurance 2009; Wilcove et al. 2013; Barnes et al. 2014). Agricultural area expansion is one of the main drivers of deforestation (Gibbs et al. 2010), and demand for agricultural output will further increase due to population and income growth. In addition to food, global demand for feed, fuel, and other biomassderived renewable resources will grow substantially over the coming decades (Alexandratos \& Bruinsma 2012; Valin et al. 2014). These developments threaten the conservation of the remaining tropical forest (Laurance et al. 2014). Increasing agricultural yields on the land already cultivated, through higher input intensity and use of better technology, could be one important way to meet the rising demand and reduce further deforestation (Green et al. 2005; Ewers et al. 2009; Phalan et al. 2011a; Stevenson et al. 2013). To be sure, agricultural intensification is not a magic bullet to conserve tropical forest and related ecosystem functions (Steffan-Dewenter et al. 2007; Perfecto \& Vandermeer 2010; Tscharntke et al. 2012). Effects will vary with the type of intensification and also with the institutional and policy context in a particular setting. Better knowledge is required about how land-sparing agricultural intensification can be implemented locally, and why past efforts have often failed. Empirical research in this direction is scant.

Here, we propose that land property rights are fundamental for agricultural production and deforestation outcomes. Land is the main source of farmers' livelihoods and also a

\footnotetext{
${ }^{3}$ This essay was published as: Kubitza, C.; Krishna, V. V.; Urban, K.; Alamsyah, Z. \& Qaim, M. (2018). Land Property Rights, Agricultural Intensification, and Deforestation in Indonesia. Ecological Economics 146, 312-321. https://doi.org/10.1016/j.ecolecon.2018.01.021 CK developed the research idea, compiled the data, conducted the regression analyses, and wrote the first draft of the paper. KU compiled the remote sensing data. KU, VVK, and MQ contributed to designing the study and preparing the data sets. All authors commented on data analysis, result interpretation, and revising the paper.
} 
major mean for accumulating and inheriting wealth. The institutions shaping access, use, and transfer of land are hence central for farmers' decision-making (Deininger \& Feder 2001). Ownership regulations for forest land and for agricultural land often differ. The available literature on the links between land property rights and deforestation focuses primarily on the effects of secure tenure for forest land (Araujo et al. 2009; Damnyag et al. 2012; Liscow 2013; Robinson et al. 2014). For agricultural land, studies have analyzed effects of tenure security on input intensity and crop productivity (Deininger et al. 2011; Fenske 2011; Bellemare 2013), yet without linking this to potential deforestation outcomes. To address this gap, we use comprehensive data from Sumatra, Indonesia, one of the hotspots of recent rainforest loss due to agricultural area expansion (Margono et al. 2014; Gatto et al. 2015; Clough et al. 2016). Data from a farm household survey, a village survey, soil samples and satellite imageries are combined to examine relationships between land ownership rights, agricultural production intensity, and farm size expansion into forest areas.

In Indonesia, small farms as well as large logging and agribusiness companies contribute to deforestation (Rudel et al. 2009b; Cacho et al. 2014). Overall, the share of land deforested by companies is larger than the share of land deforested by smallholder farmers. While precise data are not available, smallholders may have contributed less than $20 \%$ to overall deforestation in Indonesia in recent decades (Lee et al. 2014). However, there are at least two reasons why a focus on small farms - as taken in this study - is relevant nevertheless from a policy perspective. First, in Indonesia the role of smallholders in cultivating plantation crops, such as oil palm and rubber, continues to grow (Euler et al. 2017). Second, deforestation by smallholder farms is more difficult to monitor and control (Krishna et al. 2017b). Whereas large companies usually operate based on government concessions, smallholder decisions to clear forest land are individual responses to various incentives and constraints. Such behavioral responses need to be better understood, in order to design and implement effective policies.

For private farms, land titles can increase agricultural intensity and productivity through three effects (Feder \& Feeny 1991; Besley 1995; Deininger et al. 2011). First, the assurance effect, incentivizing higher investment because farmers are more secure to also reap the benefits from long-term measures to improve land quality and yield potential. Second, the collateralization effect, allowing better access to investment capital because 
land titles can be used as collateral in formal credit markets. Third, the realizability effect, resulting from more efficient land allocation given that titled land facilitates land market transactions. The empirical literature largely confirms these effects (Banerjee et al. 2002; Goldstein \& Udry 2008; Holden et al. 2009; Deininger et al. 2011; Fenske 2011; Grimm \& Klasen 2015; Lawry et al. 2016), although in some cases the influence of land titling was found to be insignificant (Quisumbing \& Otsuka; Brasselle et al. 2002; Jacoby \& Minten 2007; Bellemare 2013).

An increase in farm productivity induced through land titles could reduce deforestation (Angelsen \& Kaimowitz 2001). Higher output from the already cultivated land reduces the pressure to convert additional forest land. Also, a more productive agricultural sector could spur broader economic development, reducing population growth, enhancing nonagricultural income opportunities for rural households, and improving land-governance capacities and institutions. Empirical evidence for these types of effects is scarce, although a few studies show indeed that higher farm productivity can help spare natural habitat from agricultural conversion (Barbier \& Burgess 1997; Ewers et al. 2009; Phalan et al. 2011b). On the other hand, agricultural productivity growth could also be associated with higher rates of deforestation, for instance, by increasing the cost of forest conservation programs or by stimulating in-migration and road infrastructure investments in rural areas (Maertens et al. 2006; Phelps et al. 2013). Better understanding the complexities in concrete situations can help design appropriate policies aimed at promoting more sustainable development.

In Indonesia, much of the land that farmers use is not formally titled (Krishna et al. 2017b). Privately owned land can be titled, but the costs for farmers are relatively high. Additionally, farmers located close to the forest suffer from ambiguous ownership structures. Most of the forest land is formally owned by the state and not eligible for private titling (Agrawal et al. 2008). But the boundaries are not always clear-cut. Some of the land that farmers have cultivated for long officially counts as forest land. Moreover, local communities have customary claims and deforest land even when the newly obtained plots cannot be titled (Resosudarmo et al. 2014). The motivation to deforest will likely increase when farmers have no titles for their already cultivated land and therefore limited ability and incentives to intensify production.

To answer the question whether providing secure titles for agricultural land could help to reduce deforestation, two sub-questions will have to be addressed. First, do land titles 
increase agricultural intensity and productivity? Second, does higher productivity on the already cultivated land reduce farmers' incentives to clear additional forest land? The first sub-question will be addressed by comparing input use and crop productivity on farms with and without land titles and controlling for other relevant factors. The second subquestion is less straightforward to answer, because this would require farm-level data on crop productivity in the past, which we do not have. However, we address this subquestion indirectly by analyzing the relationship between the possession of land titles, historical forest coverage, deforestation activities of farm households, and farm size in a spatially explicit way. In addition, we look at the association between current crop productivity and farm size, which - together with the other results - may allow some cautious conclusions on the role of land titles for deforestation and the underlying mechanisms.

\subsection{Data}

\subsubsection{Socioeconomic data}

This research builds on data collected in Jambi Province on the island of Sumatra, Indonesia. Jambi has been one of the regions with rapid loss of tropical rainforest over the last few decades. Forest cover in Jambi declined from 48\% in 1990 to 30\% in 2013 (Drescher et al. 2016). Nevertheless, 43\% of Jambi's total area was officially categorized as state forest in 2000 (Komarudin et al. 2008). Agricultural production in Jambi is dominated by plantation crops, especially rubber (Hevea brasiliensis) and oil palm (Elaeis guineensis). Rubber is primarily grown by local farmers with only some involvement of large-scale companies. Companies are more involved in oil palm, but even in oil palm more than $40 \%$ of the area is cultivated by smallholder farmers (Euler et al. 2017). That smallholders contribute to deforestation in Jambi in a significant way was underlined in a recent study (Krishna et al. 2017b), which showed that $18 \%$ of the rubber and oil palm plots cultivated by smallholders were acquired through direct forest appropriation.

A survey of farm households was conducted in Jambi in two rounds, 2012 and 2015, as part of a larger interdisciplinary research project (Drescher et al. 2016). A multi-stage sampling framework was used to obtain a representative sample of local farm households. 
At the first stage, five regencies of Jambi located in tropical lowland rainforest areas were selected. At the second stage, a total of 40 villages were randomly selected in these five regencies. In addition, five villages, where more intensive measurements by other teams of the same research project were ongoing (Drescher et al. 2016), were purposively selected, resulting in a total of 45 villages. In these villages, 700 households were randomly selected proportional to village size. There are two types of villages in Jambi, autochthonous and transmigrant villages. Transmigrant villages were established as part of the government's transmigration program (Gatto et al. 2017). Most households in transmigrant villages were allocated titled land by the state and started producing plantation crops under contract with one of the large public or private companies. Hence, the institutional and agricultural production conditions are quite different. In this research, we only consider the 34 autochthonous villages in the sample, with 473 farm household observations in 2015 (and 471 household observations in 2012). Out of these, around 25\% are migrants (Table A1 in the Online Appendix), but these migrants in autochthonous villages did not come as part of the government's transmigration program (Gatto et al. 2015). Most of the households in the two survey rounds are identical. The attrition rate between 2012 and 2015 was $6 \%$. Households that could not be surveyed again in 2015 (mostly due to out-migration) were replaced with other randomly selected households in the same villages.

In both survey rounds, household heads were interviewed with a structured questionnaire, capturing a wide range of variables related to the households' socioeconomic situation and the institutional context (Euler et al. 2017; Krishna et al. 2017a). Details about the different plots owned and cultivated by the farm households were also collected. In 2015, the 473 households cultivated a total of 902 plots with plantation crops; out of these 690 were cultivated with rubber, the rest with oil palm. For all these plots, data on general plot characteristics, such as size, location, and status of land titling, were elicited. In addition, detailed input-output data were captured for all plots in 2012 and for a random sub-sample of plots in 2015. For the analysis of agricultural productivity and intensity, we concentrate on productive rubber plots (those where the trees are old enough such that rubber is already being harvested). Input-output relationships in rubber and oil palm are quite different, so combining both crops in the same models would not be useful. Besides the interviews with household heads, village representatives were interviewed in 
all sample villages to capture data on village size, ethnic composition, and other villagelevel characteristics.

\subsubsection{Soil and remote sensing data}

In the farm household survey, respondents were asked to classify the soil fertility on each of their plots as low, medium, or high. In addition to these data on perceived soil quality, soil samples were taken in 2012 for a randomly selected sub-sample of 92 rubber plots. These soil samples were taken and analyzed by a different team of researchers (Guillaume et al. 2016). We use topsoil properties, such as bulk density, carbon content, and carbon/nitrogen ratio as additional explanatory variables in the rubber production models.

Land cover maps of Jambi Province from the years 1990 and 2013 were obtained using multi-temporal Landsat TM and OLI satellite imageries with a spatial resolution of 30x30 $\mathrm{m}$. Land cover classification is based on automatic classification and additional qualitative, visual interpretation to reduce miss-classifications (Melati et al. 2014). In this research, we are particularly interested in the share of forest in the vicinity of the sample households, which we determined by evaluating land cover classifications in circles with specific radius around the households' residence. We use different alternatives with $2 \mathrm{~km}, 5 \mathrm{~km}$, and 10 $\mathrm{km}$ radius. Households with a high share of forest in their vicinity are considered as being located at the forest margins.

\subsection{Econometric methods}

The analysis is done in three steps. First, we present models that analyze the effect of land titles on agricultural productivity. Second, we use similar models to analyze effects of land titles on agricultural intensity (input use). Third, we examine spatial patterns by developing and estimating models to analyze the relationships between historic forest margin, possession of land titles, deforestation activities, and farm size. 


\subsubsection{Models to analyze agricultural productivity}

To analyze the effect of land titles on productivity in rubber, we estimate householdlevel panel regression models of the following type:

Eq. (1): $\ln \left(P R_{i t}\right)=\beta_{0}+\beta_{1} S L T_{i t}+\beta_{2} X_{i t}+\mu_{i}+\varepsilon_{i t} \quad$ (household level)

where $P R_{i t}$ is total annual rubber yield per hectare of household $i$ at time $t . S L T_{i t}$ is the share of household $i$ 's land cultivated with plantation crops that had a systematic land title at time $t$. The share can vary between 0 and $1 . X_{i t}$ is a vector of other farm and household characteristics that may also influence rubber yields, such as farm size, age, gender, and education of the household head, and a wealth index. The wealth index was constructed based on ownership of the following assets: television, different types of vehicles, refrigerator, and washing machine. A principal component analysis was used to determine the weight of each asset in the wealth index (Filmer \& Pritchett 2001). $\mu_{i}$ is the unobserved time-invariant heterogeneity of the model, while $\varepsilon_{i t}$ is the iid error term.

We also estimate similar models at the plot level:

Eq. (2): $\ln \left(P R_{\text {pit }}\right)=\beta_{0}+\beta_{1} L T_{\text {pit }}+\beta_{2} X_{i t}+\beta_{3} S_{p i t}+\mu_{p i}+\varepsilon_{p i t} \quad$ (plot level)

where $P R_{\text {pit }}$ is the annual rubber yield per hectare on plot $p$ of household $i$ at time $t$. $L T_{\text {pit }}$ is a dummy variable taking the value 1 if the plot was systematically titled at time $t$. $S_{\text {pit }}$ includes additional plot characteristics such as age of the rubber trees and variables related to plot location.

Due to the sampling framework used, households and plots are clustered at the village level. We use hence cluster-corrected standard errors (Pepper 2002; Cameron et al. 2011). For interpretation of the estimation coefficients, functional form has to be considered. $S L T_{i t}$ in Eq. (1) is a continuous variable, so that $\beta_{1}$ is interpreted as the percentage effect on rubber yield. $L T_{\text {pit }}$ in Eq. (2) is a dummy variable, so that the percentage effects is calculated as $\left\{\mathrm{e}^{\widehat{\beta_{1}}-0.5 \widehat{V}\left(\widehat{\beta}_{1}\right)}-1\right\}$ (van Garderen \& Shah 2002).

The models in Eqs. (1) and (2) are estimated with random effects (RE) panel estimators. Studies with micro-level data to assess the effects of land titling often struggle 
with endogeneity issues (Brasselle et al. 2002). Endogeneity bias occurs when unobserved characteristics are jointly correlated with land titling and crop productivity. In particular reverse causality might be a concern, since high productivity might influence farmers' decision to title their plots. For instance, if investments in plot productivity can be made which also increase tenure security, tenure security would be endogenous. However, we use land titles as a proxy for tenure security, so investments are not likely to be confounded. Clearly, having a land title may be affected by other factors related to, for example, the characteristics of the land, which may in turn affect our estimates. Valid instruments for land titles, which are exogenous and fulfill the exclusion restrictions, are usually hard to find (Fenske 2011; Bellemare 2013; Grimm \& Klasen 2015). We use different strategies to address endogeneity and reduce related bias to the extent possible. First, we include a wide range of plot- and household-level control variables to reduce the likelihood of unobserved heterogeneity. In robustness checks, we also include various measures of soil quality, which has rarely been done in previous research (Bellemare 2013). Second, in addition to using random effects, we also estimate the productivity models with fixed effects (FE) estimators and balanced plot- and household-level panel data. The variation in land titling within plots and households between 2012 and 2015 is small, but sufficient to obtain FE estimates. We use the Hausman test (Wooldridge 2002) to compare between the RE and FE models (Table A2). Test results fail to reject the hypothesis that the RE models produce consistent estimates. Third, in addition to model estimates with all observations, we split the sample into migrants and non-migrants and estimate separate models for these two groups. We expect heterogeneous impacts of land titling, because customary land claims that apply to autochthonous people do not apply to migrants from outside the region. 


\subsubsection{Models to analyze agricultural intensity}

To analyze the effect of land titles on intensity of rubber production, we estimate plotlevel panel regression models of the following type:

$$
\begin{array}{ll}
\text { Eq. (3): } I N V_{p i t}=\beta_{0}+\beta_{1} L T_{p i t}+\beta_{2} X_{i t}+\beta_{3} S_{p i t}+\mu_{p i}+\varepsilon_{p i t} & \text { (plot level) } \\
\text { Eq. (4): } \ln (L S)_{\text {pit }}=\beta_{0}+\beta_{1} L T_{p i t}+\beta_{2} X_{i t}+\beta_{3} S_{p i t}+\mu_{p i}+\varepsilon_{p i t} & \text { (plot level) }
\end{array}
$$

where $I N V_{\text {pit }}$ is total annual expenditures on material inputs applied per hectare (ha) on plot $p$ by household $i$ at time $t$. Material inputs include chemical fertilizers and pesticides (incl. herbicides). $L S_{\text {pit }}$ is annual labor input (incl. family and hired labor) measured in hours per ha. The other variables are defined as above. Since more than $50 \%$ of the sample farmers did not use any material inputs during the survey years, we do not take logs of $I N V_{\text {pit }}$. Given censoring of the dependent variable at 0 , we use a Tobit specification for the model in Eq. (3). To test the effect of $I N V_{\text {pit }}$ and $L S_{\text {pit }}$ on crop productivity, we also estimate additional specifications of Eq. (2) with these inputs included as explanatory variables.

\subsubsection{Spatial regression models}

To estimate the effect of historical forest closeness on the probability of holding a land title, we estimate the following plot-level probit model:

Eq. (5): $P\left(L T_{p i v}\right)=\beta_{0}+\beta_{1} F_{i v}+\beta_{2} Z_{p i v}+\beta_{3} Z_{i v}+\beta_{4} Z_{v}+\varepsilon_{p i v} \quad$ (plot level)

where $L T_{\text {piv }}$ is a dummy indicating whether or not plot $p$ of household $i$ in village $v$ was systematically titled in 2015, and $F_{i v}$ is the share of forest land in 1990 in a circle with specific radius around the household residence. $F_{i v}$ can take values between 0 (no forest in 1990) to 1 (completely forested in 1990). The reference year 1990 was chosen because most of the formal land classifications in Indonesia took place in the 1980s (Indrarto et al. 2012). We estimate separate models, using radii of $2 \mathrm{~km}, 5 \mathrm{~km}$, and $10 \mathrm{~km}$ to construct $F_{i v}$. 
In each of these models, plots that are located outside the specific radius are excluded from estimation. A further robustness check is performed, replacing $F_{i v}$ with a binary variable indicating if the plot was acquired by the household through deforestation. $Z_{p i v}, Z_{i v}$, and $Z_{v}$ are further plot-, household-, and village-level controls. Eq. (5) includes both rubber and oil palm plots.

It is likely that land titling is also affected by spatial factors such as local policies, distances to roads and markets, or environmental conditions. This can possibly lead to spatial dependence in the models in Eq. (5). All models were tested for spatial autocorrelation using Moran's I, Anselin's, and Florax's Lagrange Multiplier tests (Baltagi 2003). These tests failed to reject the hypothesis of zero spatial autocorrelation. For completeness, spatial lag and spatial error models are reported in Table A3.

We hypothesize that households close to the forest are less likely to hold land titles and therefore have stronger incentives to expand their farms into the forest. After controlling for other factors, this should lead to larger farm sizes at the forest margins. To test this hypothesis, we regress farm size in 2015 on forest closeness in 1990 and a set of control variables. Again, we used Moran's I, Anselin's, and Florax's Lagrange Multiplier tests (Baltagi 2003) to test for spatial autocorrelation. These tests reject the hypothesis of zero spatial autocorrelation. Based on the test results, we estimate spatial lag models of the following type:

Eq.(6): $\ln \left(F S_{i v}\right)=\rho W \ln \left(F S_{i v}\right)+\beta_{0}+\beta_{1} F_{i v}+\beta_{2} V_{i v}+\beta_{3} V_{v}+\varepsilon_{i v}$ (household level)

where $F S_{i v}$ is total farm size of household $i$ in village $v$ measured in hectares, $F_{i v}$ is the share of forest land in 1990 (as defined above). $V_{i v}$ and $V_{v}$ are household- and villagelevel controls. $W$ is an $\mathrm{N} \times \mathrm{N}$ spatial weights matrix ( $\mathrm{N}=$ Number of households) based on the inverse Euclidian distance between the households' residence. The parameter $\rho$ measures the degree of spatial correlation. $W$ is row standardized, such that for each $i$, $\sum_{j} w_{i j}=1$ (Baltagi 2003). The spatial lag $\rho W \ln \left(F S_{i v}\right)$ can be interpreted as a weighted average of the farm sizes of neighboring households. For comparison, spatial error and ordinary least squares models are reported in Table A4. 


\subsection{Results}

\subsubsection{Descriptive statistics}

The average size of farms in our sample in 2015 was around 4 ha. This refers to the land cultivated, regardless of whether or not the farmer formally owns the different plots. Locations of the farm households are depicted in Figure 1 (Maps 1 and 2). Responses during the survey interviews suggest that households are actively engaged in deforestation. This is also confirmed by land cover maps. In 1990, about $17 \%$ of the area within a $5 \mathrm{~km}$ radius around farmers' residence was covered with forest; by 2013, this forest share was reduced to $3 \%$. Much of the previous forest land is now grown with rubber and oil palm. Even though the area cultivated with oil palm grew faster during the last two decades (Gatto et al. 2015), rubber remains the dominant crop in the study region. About $30 \%$ of the sample farms grow oil palm, whereas $86 \%$ grow rubber (Table A1). This is also the reason why we focus on rubber for the analysis of crop productivity and production intensity.

Concerning supply chains in the local rubber sector, famers mostly sell their harvest to traders in the village, who then transport the rubber to the processing factories (Kopp \& Brümmer 2017). Fertilizer, pesticides, and other material inputs can often be bought from the village traders. Alternatively, inputs can be purchased in the next market, which is 5-6 $\mathrm{km}$ away from the households' residence on average (Table A1). Given relatively bad infrastructure conditions in large parts of Jambi, transportation and transaction costs can be substantial, meaning that farm-gate input and output prices are influenced significantly by distance to roads and markets. 
Figure 1: Maps of land uses in Jambi Province (Sumatra) in 1990 and 2013
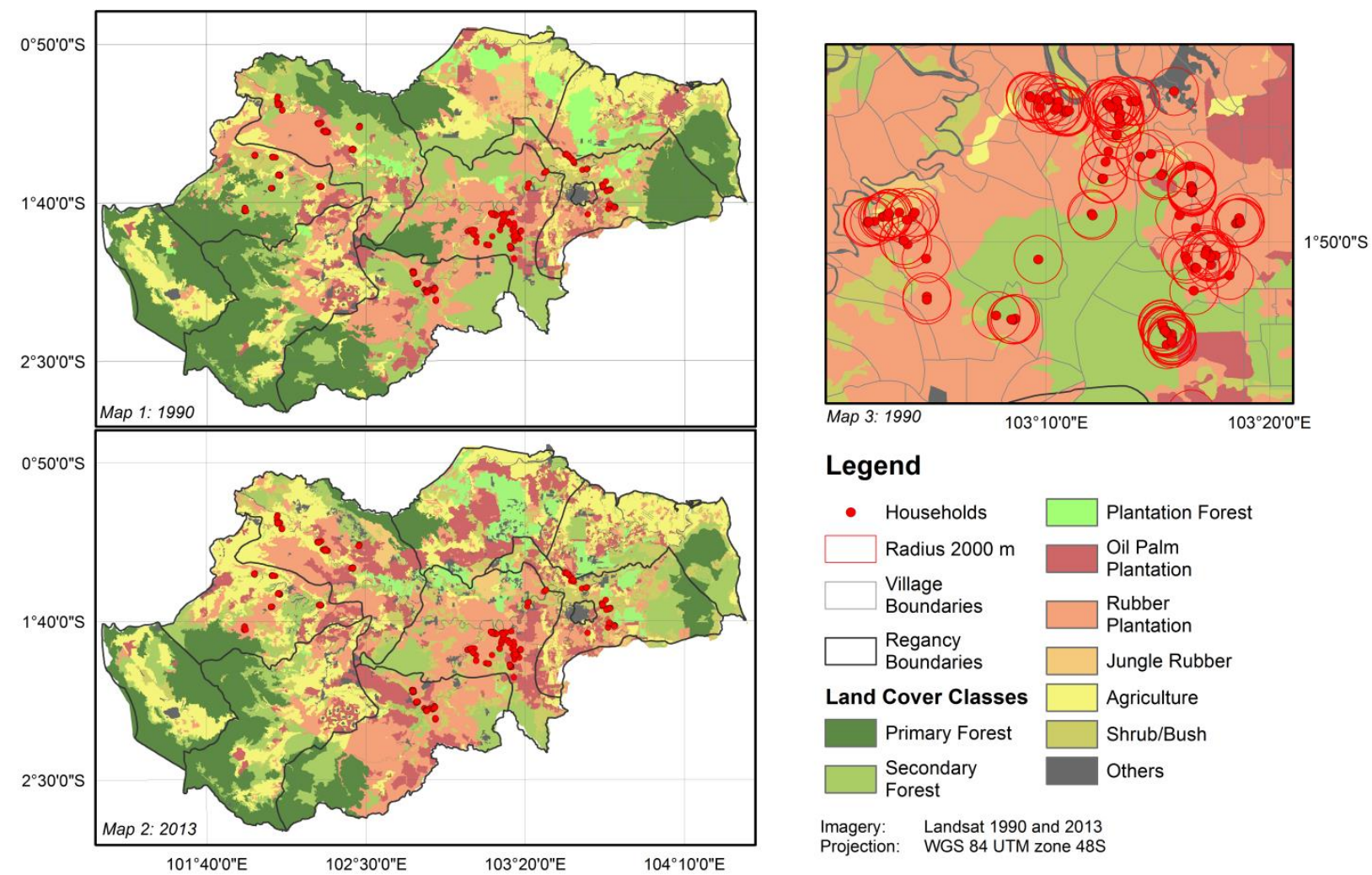

Notes: Maps 1 and 2 depict Jambi Province in 1990 and 2013. Map 3 is one example from a sub-region (Harapan Rainforest) with eight sample villages in 1990. The red circles indicate a $2 \mathrm{~km}$ radius around the sample households' residence. Circles with different radius $(2,5,10 \mathrm{~km})$ were used to calculate the share of forest land around households.

Most of the plots that sample farmers cultivate are not formally titled, but held under customary tenure. In 2015 , only $10 \%$ of the rubber plots had a systematic land title, which is a document that all formal authorities recognize. Figure 2 shows that systematic land titling is a rather recent phenomenon in the study area, largely due to changes in formal land policies. Since the late-1990s, the Indonesian government has intensified its efforts to facilitate the systematic titling of land in agricultural use (Krishna et al. 2017b). Note that the titling of land in a particular year is not an indication of new land acquisition in that same year. In most cases, plots were systematically titled many years after their acquisition. In addition to systematic land titles, so-called sporadic land titles exist, which are cheaper for farmers to obtain but only recognized by local authorities and thus of limited value as collateral in formal credit markets (Kunz et al. 2016; Krishna et al. 2017b). About $22 \%$ of the rubber plots have a sporadic land title (Table A1). 
Figure 2: Land titling and deforestation activities by farmers (1965-2015)

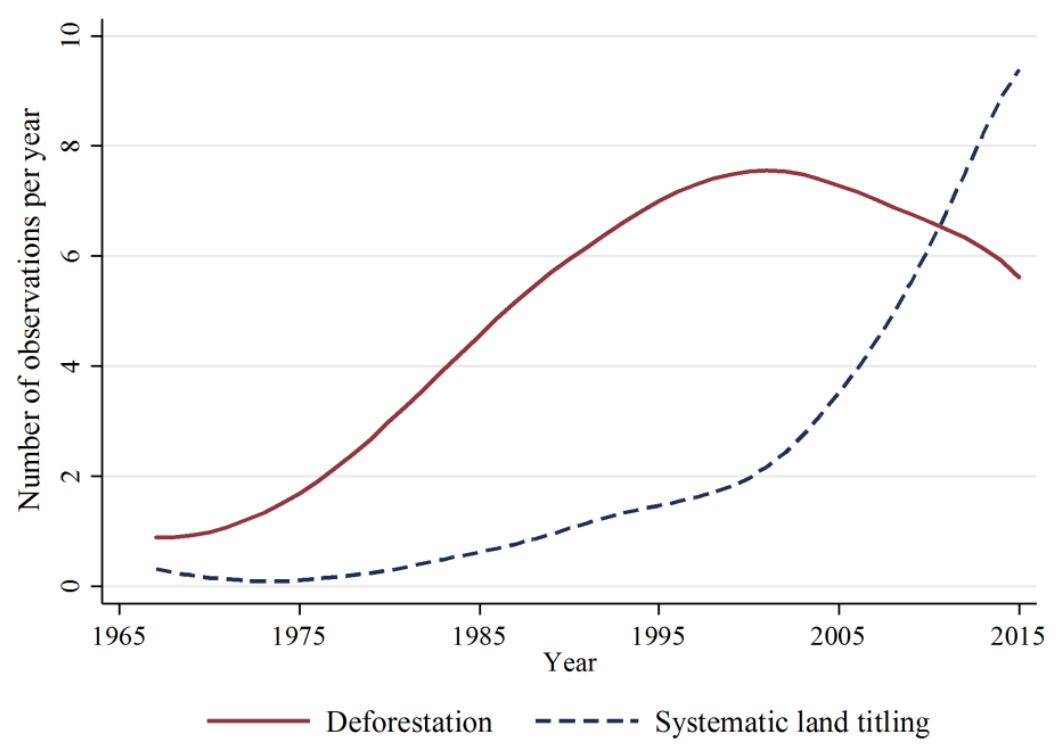

Notes: Based on farmer recall data from 902 plots. The graph shows the number of plots that were obtained through deforestation and the number of plots for which farmers obtained a systematic land title in a particular year. The curves were constructed using locally-weighted time series smoothing.

Figure 2 also shows deforestation activities by sample farmers in terms of the number of plots acquired through forest clearing in a particular year. The frequency of deforestation has declined since the late-1990s, which may be due to forest land becoming scarcer in Jambi. The rising provision of systematic titles for the land already cultivated may also have played a role. This is analyzed more formally below. The peak in deforestation around the year 2000 is possibly also related to the political turbulences during the so-called Reformasi era and the subsequent decentralization process.

\subsubsection{Land titles and agricultural productivity}

To analyze whether land property rights have an effect on agricultural productivity, we estimated regression models with rubber yield as dependent variable and land titles as explanatory variables, as explained in Eqs. (1) and (2). The main results are shown in Table 1 (full model results with all explanatory variables are shown in Table A2 in the Online Appendix). In all model specifications, systematic land titles have positive and significant coefficients, while sporadic land titles have insignificant effects. In the household-level models, the different rubber plots of a household are combined. Compared 
to a situation with no land titles, systematic titling of all plots (share of land with systematic title equal to 1) leads to an increase in crop productivity by $35 \%$ (column 1 ). In column (2), we only include households that migrated to the villages from outside the region. For these households, the productivity effect of systematic land titles is even larger. It is not unexpected that migrants benefit more from land titles. First, migrants often belong to a different ethnicity than autochthonous households. Given smaller family networks in the local context, migrants depend more on formal credit markets to access financial capital (collateralization effect). Second, for migrants, customary land claims do not hold, so that formal property rights play a more important role for tenure security (assurance effect).

In the plot-level models in Table 1, each of the rubber plots is considered separately. Plots with a systematic land title have $15 \%$ higher yields than plots without title (column 3). The effect is smaller than in the household-level models. This is plausible, because the same household can have titled and untitled plots, so that spillovers may occur. For instance, a title for one plot will usually suffice as collateral to obtain a credit to pay for farm inputs that can be used to increase productivity on all of the household' plots. Also in the plot-level specifications, the effect for migrants (column 4) is larger than the effect for the total sample of farmers.

While we control for plot- and household-level characteristics, including road distance that influences farmers' access to input and output markets (see Table A2), it is still possible that there are unobserved factors that influence land titling and productivity simultaneously. Such unobserved heterogeneity could lead to bias in the coefficient estimates. For instance, land with better soil quality will result in higher yields and may also have a higher likelihood to be titled. The measures of perceived soil quality ${ }^{4}$ are included in the model in column (5) of Table 1. In addition, column (6) shows precise soil quality measurements as explanatory variables for the random sub-sample for which these measurements are available. In both these models, the coefficient for systematic land titles remains positive and significant. As soil quality may also be correlated with other relevant unobserved factors, this suggests that the finding of a positive effect of land titles on crop productivity is rather robust to unobserved heterogeneity.

\footnotetext{
${ }^{4}$ Number of observations are lower than in the full model (Table 1 col. (3)) due to missing data.
} 


\subsubsection{Land titles and agricultural intensity}

Estimation results with indicators of input intensity as dependent variables, as explained in Eqs. (3) and (4), are summarized in Table 2 (full results are shown in Table A5). Possession of systematic land titles significantly increases the use of material inputs (chemical fertilizers and pesticides). The marginal effect is 114 thousand IDR/ha in column (1). Among migrant farmers, the effect is even larger (column 2). For labor input (column 3), we also find a positive effect of systematic land titles, which is somewhat smaller $(13 \%)$ than that for material inputs. For migrant farmers, the effect of systematic land titles on labor is insignificant (column 4). On the other hand, sporadic land titles seem to increase labor input among migrants. As mentioned, sporadic titles are of limited value in formal credit markets, but - unlike material inputs - farmers rarely take a credit to pay for hired labor.

We expect that the effect of land titles on agricultural productivity is partly channeled through higher input intensity. Indeed, when including input use in the productivity model (columns 5 and 6 in Table 2), material and labor inputs both have significantly positive effects, whereas the effect of systematic land titles on productivity declines (compare with column 3 in Table 1$)^{5}$. However, the land title effect remains positive and significant, suggesting that other transmission channels also play an important role.

\subsubsection{Spatial patterns of land titling}

Now we take a spatial perspective and analyze the likelihood of plots being titled as a function of forest closeness (see Eq. 5). As mentioned, plots located in areas designated as state forest are not eligible for titling, even though the boundaries are not clear-cut. Table 3 shows plot-level probit regression estimates with a dummy for systematic land titles in 2015 as dependent variable and the share of forest in 1990 as explanatory variable (column 1-3). Controlling for other factors (see full results in Table A6), location at forest margins (areas that were more forested in the past) decreases the likelihood of systematic land titling by 13-18 percentage points. Column (4) in Table 3 shows a model with a somewhat

\footnotetext{
${ }^{5}$ Note that the number of observations is declining slightly due to missing data.
} 
different specification, confirming that plots that were deforested by households themselves are less likely to be titled ${ }^{6}$.

Concerning the effects of control variables, we find that plots located further away from roads are significantly less likely to be titled. On the other hand, plots that were already used by the farmer for a longer period of time are more likely to be titled. In some of the models, we also find a significantly positive effect of household wealth on land titling (Table A6).

Without land titles, farmers at the forest margins are less able and willing to increase productivity, so they may have stronger incentives to increase their farm size by further expanding into forest land. To test this hypothesis, we regress farm size in 2015 on the share of forest in 1990 (see Eq. 6). The estimation results are shown in columns (5) to (7) of Table 3. As expected, farms at the forest margins are significantly larger than farms further away from the forest. The model in column (8) of Table 3 also confirms that households' deforestation activities have directly contributed to larger farm sizes. ${ }^{7}$

We emphasize that lack of land titles is not the only factor that could explain larger farms at forest margins. Closeness to the forest is likely correlated with the private costs of deforestation: for farmers close to the forest it may be cheaper to deforest due to lower transportation costs. This means that the provision of land titles may not completely eliminate deforestation, but it would still influence incentive structures towards more intensive forms of production. For farms close to the forest, additional incentives for more intensive forms of production seem to be particularly important to reduce deforestation activities. Spatial dependencies are accounted for in our analysis through the use of spatial error models and spatial lag models, as explained above.

While we have no plot- or farm-level data on cropping patterns before 2012, which could help to elucidate historical relationships between intensity, productivity, and farm size expansion, in Table A7 we regress current farm size (in 2015) on current rubber yields. Farm size is negatively associated with yield, even after controlling for other factors, providing further evidence that higher productivity may indeed reduce the incentive to expand the area cultivated.

\footnotetext{
${ }^{6}$ The binary variable indicates if a plot was directly encroached from forest versus being purchased for a sub-sample of plots with available information.

${ }^{7}$ Since we are not interested here in direct or indirect effects of explanatory variables but rather to assess if a significant association between farm size and being located at former forest margins exists, we report parameter estimates of $\beta$.
} 
Table 1: Land titles and agricultural productivity

\begin{tabular}{|c|c|c|c|c|c|c|}
\hline & \multicolumn{2}{|c|}{ Household-level models } & \multicolumn{2}{|c|}{ Plot-level models } & \multicolumn{2}{|c|}{$\begin{array}{l}\text { Plot-level models with soil quality } \\
\text { controls }\end{array}$} \\
\hline & (1) & (2) & (3) & (4) & $(5)$ & $(6)$ \\
\hline & Full sample & Migrants & Full sample & Migrants & Full sample & $\begin{array}{c}\text { Sub-sample } \\
\text { with soil quality } \\
\text { measures }\end{array}$ \\
\hline \multirow[t]{2}{*}{ Share of land with systematic title } & $0.351^{* * * *}$ & $0.586^{* * * *}$ & $0.152^{* * *}$ & $0.370^{* * * *}$ & $0.183^{* *}$ & $0.697^{* * * *}$ \\
\hline & $(0.085)$ & $(0.107)$ & $(0.063)$ & $(0.098)$ & $(0.071)$ & $(0.265)$ \\
\hline \multirow[t]{2}{*}{ Share of land with sporadic title } & 0.019 & 0.111 & -0.017 & 0.039 & -0.036 & -0.131 \\
\hline & $(0.071)$ & $(0.090)$ & $(0.071)$ & $(0.073)$ & $(0.079)$ & $(0.254)$ \\
\hline \multirow[t]{2}{*}{ Size of rubber area (ha) } & $-0.030^{*}$ & -0.006 & $-0.086^{* * * *}$ & $-0.132^{* * * *}$ & $-0.088^{* * * *}$ & $-0.097^{*}$ \\
\hline & $(0.016)$ & $(0.028)$ & $(0.017)$ & $(0.029)$ & $(0.021)$ & $(0.049)$ \\
\hline \multirow[t]{2}{*}{ Wealth index (quintiles) } & 0.011 & -0.023 & $0.031^{* *}$ & 0.021 & $0.035^{*}$ & $0.134^{* * * *}$ \\
\hline & $(0.017)$ & $(0.031)$ & $(0.015)$ & $(0.021)$ & $(0.019)$ & $(0.049)$ \\
\hline Perceived soil quality included & No & No & No & No & Yes & No \\
\hline Soil quality measurements included & No & No & No & No & No & Yes \\
\hline Chi2 / F- statistic & $297.453^{* * *}$ & $232.371^{* * * *}$ & $312.312^{* * * * *}$ & $2332.550^{* * * *}$ & $485.131^{* * *}$ & $3.63^{* * * *}$ \\
\hline Number of observations & 665 & 174 & 851 & 231 & 741 & 92 \\
\hline
\end{tabular}


Table 2: Land titles and agricultural intensity

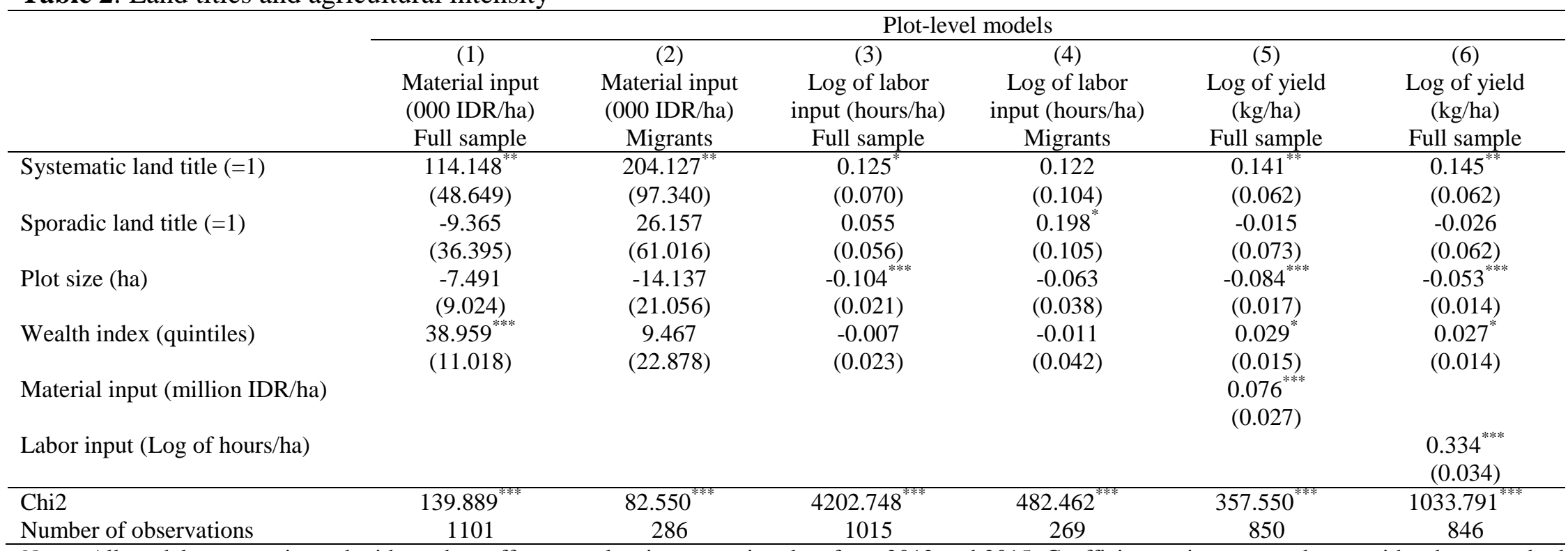

Notes: All models were estimated with random effects panel estimators using data from 2012 and 2015. Coefficient estimates are shown with robust standard errors clustered at village level in parentheses. Due to left-censoring of the dependent variable, a Tobit specification was used in columns (1) and (2). The coefficients themselves measure how the latent variable changes with respect to changes in the regressors. IDR, Indonesian rupiah. Additional covariates that were included in estimation are shown in Table A5. ${ }^{*} p \leq 0.10,{ }^{* *} p \leq 0.05,{ }^{* * *} p \leq 0.01$. 
Table 3: Historical forest coverage, land titles, and farm size

\begin{tabular}{|c|c|c|c|c|c|c|c|c|}
\hline & \multicolumn{4}{|c|}{ Plot-level models (systematic land title $=1$ ) } & \multicolumn{4}{|c|}{ Household-level models (log of farm size in ha) } \\
\hline & (1) & $(2)$ & $(3)$ & (4) & $(5)$ & $(6)$ & $(7)$ & $(8)$ \\
\hline & $\begin{array}{l}2 \mathrm{~km} \\
\text { radius }\end{array}$ & $\begin{array}{l}5 \mathrm{~km} \\
\text { radius }\end{array}$ & $\begin{array}{l}10 \mathrm{~km} \\
\text { radius }\end{array}$ & All plots & $\begin{array}{l}2 \mathrm{~km} \\
\text { radius }\end{array}$ & $\begin{array}{l}5 \mathrm{~km} \\
\text { radius }\end{array}$ & $\begin{array}{l}10 \mathrm{~km} \\
\text { radius }\end{array}$ & All plots \\
\hline Share of forested area in 1990 & $\begin{array}{c}-0.180^{\text {**** }} \\
(0.057)\end{array}$ & $\begin{array}{l}-0.128^{* *} \\
(0.050)\end{array}$ & $\begin{array}{c}-0.180^{* * * *} \\
(0.065)\end{array}$ & & $\begin{array}{c}0.268^{*} \\
(0.146)\end{array}$ & $\begin{array}{l}0.337^{\text {** }} \\
(0.155)\end{array}$ & $\begin{array}{l}0.453^{* *} \\
(0.198)\end{array}$ & \\
\hline Deforestation $(=1)$ & & & & $\begin{array}{c}-0.060^{* *} \\
(0.028)\end{array}$ & & & & $\begin{array}{c}0.258^{* * * *} \\
(0.086)\end{array}$ \\
\hline Wealth index (initial, quintiles) & 0.003 & 0.009 & $0.018^{*}$ & $0.029^{* *}$ & $0.142^{* * *}$ & $0.144^{* * *}$ & $0.146^{* * *}$ & $0.140^{* * * *}$ \\
\hline & $(0.015)$ & $(0.012)$ & $(0.010)$ & $(0.011)$ & $(0.034)$ & $(0.034)$ & $(0.034)$ & $(0.034)$ \\
\hline Migrant (=1) & -0.042 & -0.020 & 0.009 & -0.014 & 0.137 & 0.135 & 0.132 & 0.148 \\
\hline & $(0.035)$ & $(0.030)$ & $(0.025)$ & $(0.029)$ & $(0.101)$ & $(0.101)$ & $(0.101)$ & $(0.101)$ \\
\hline Wald chi2 / squared correlation & $74.830^{* * * *}$ & $95.021^{* * * *}$ & $77.205^{* * * *}$ & $75.126^{* * *}$ & 0.208 & 0.210 & 0.211 & 0.217 \\
\hline Number of observations & 433 & 660 & 750 & 594 & 462 & 462 & 462 & 462 \\
\hline
\end{tabular}




\subsection{Discussion}

Using data from farm households in Jambi Province, Sumatra, we have shown that secure land property rights contribute to higher agricultural intensity and productivity. Higher productivity on the land already cultivated can lower the need to convert additional forest land and thus reduce deforestation. Yet, the effectiveness of this mechanism depends on the spatial patterns of land titling and intensification. While it is particularly important that farmers at the forest margins have secure land property rights, our data have revealed that farmers close to historically forested areas are unlikely to hold formal land titles. Like many other developing countries (Agrawal et al. 2008), Indonesia considers forest land as state property. However, forest governance is constrained by unclear boundaries, limited capacity to monitor, and overlaps of state and customary land claims (Indrarto et al. 2012). While land that was deforested in violation of state law is not eligible for titling, farmers are rarely prosecuted and punished for deforestation activities. Without land titles, farmers at the forest margins have little incentive to intensify and rather expand their farms by deforesting additional land. Indeed, farms at the historic forest margins were found to be larger in size.

The results suggest that the provision of land titles can contribute to agricultural intensification and reduced deforestation, even though this potential is not fully realized in this particular setting. Addressing the existing inconsistencies between state and customary land institutions at the forest margins would be important to encourage land-sparing agricultural intensification. This does not mean that farmers encroaching forest land should easily be granted land titles for the newly deforested plots. But a regime that does not effectively impede deforestation and at the same time excludes farmers at the forest margins from the legal property system is probably the worst recipe for forest protection and agricultural development. Besides improving farmer's access to land titles for nonforest land, better recognition of customary land rights and more effective protection of forest land without recognized claims could be useful policy responses.

Our research coincides with major efforts of the Indonesian government to reform its land governance system. After a court ruling in 2013, the government is now negotiating major releases of land to local communities through social forestry schemes. Moreover, the government aims to legalize and reallocate land ownership to local families through the so- 
called TORA initiative (Land Object of Agrarian Reform) (LANDac 2016). Our research delivers timely support for the TORA initiative in particular, because the land covered in our survey is mostly cultivated by individual farming families. However, privately-owned agricultural land with secure land titles and community-owned forest land is not a contradiction. Our results suggest that this combination can contribute to efficient agricultural production on the already cultivated land, while effectively preserving the remaining forest resources.

We acknowledge that the relationships are complex and that we were not able to establish all relevant effects unambiguously. Further research is required to confirm some of the mechanisms. First, our study concentrated on deforestation activities by smallholder farmers. Large logging and agribusiness companies also contribute to deforestation in a significant way, and the incentive structures in the company sector are likely different. Second, we did not show that farm location at the forest margins affects agricultural productivity and intensity directly. The reason is that forest closeness is correlated with many unobserved factors that could also influence yield. Beyond soil characteristics, microclimate and the abundance of various types of organisms may play important roles (Guillaume et al. 2016).

A third aspect that we did not analyze explicitly is that higher agricultural productivity may lead to higher land rents, which could make further forest conversion more attractive for outside agents and thus induce in-migration. However, another recent study with data from Jambi showed that autochthonous farm households are much more involved in deforestation than migrant households (Krishna et al. 2017b). In any case, to avoid that the increasing agricultural productivity threatens forest resources, it will be useful to invest some of the accruing economic benefits into effective forest protection. Fourth, higher use of material inputs and technologies may possibly lead to a substitution of capital for manual labor, with the freed labor becoming available to deforest and cultivate additional land. However, higher fertilizer use tends to increases labor demand. Indeed, our data show that land titles have an increasing effect on labor input on the already cultivated plots. Another material input that is used more widely by farmers with land titles is herbicides, which could be labor-saving in general. Yet, the labor input for manual weeding in this setting is small, so that increasing herbicide use leads to better weed control and higher yields rather than significant reduction in the use of manual labor. We acknowledge that if 
farm households had substantial labor surplus, it would still be economically rational for them to use the surplus labor for deforestation. However, farming households in Jambi are typically rather labor-constrained, which is also why many of them are currently switching from rubber to oil palm, a crop with significantly lower labor requirements (Euler et al. 2017; Krishna et al. 2017a).

Two seemingly contrasting agricultural options for environmental conservation are widely discussed: extensive farming with higher levels of ecological functions but also higher land demand, and intensive farming with lower levels of ecological functions and lower land demand (Green et al. 2005; Rudel et al. 2009a; Tscharntke et al. 2012). Which of these options is preferable is highly context-specific. Different settings and different valuations of ecosystem functions can produce a wide range of optimal land allocations and degrees of intensity (Steffan-Dewenter et al. 2007). In tropical rainforest areas, as analyzed here, highly-productive farming with lower land demand and effective forest protection could possibly be the best option to promote sustainable development. The reason is that no agricultural system is able to sustain the same level of biodiversity and other ecosystem functions as provided by tropical rainforest (Burney et al. 2010; Clough et al. 2016).

Sumatra had experienced significant deforestation even before land titling started. Hence, from today's perspective the question whether land titles could have reduced deforestation is partly hypothetical. Our findings could help to improve the conservation of the remaining forest land in Sumatra. More importantly, however, our results can provide important insights for other regions as well. Many countries with tropical rainforests face similar complications with smallholder farmers encroaching forest land to expand the agricultural area (Meyfroidt et al. 2013; Godar et al. 2014). In such situations, partly switching from costly control and sanction mechanisms to more incentive-based policies seems to be promising. This study has made an attempt to contribute in this direction. 
Data Availability: The data used in this study are archived with openly accessible, keyword-searchable metadata and data holder contact details for data requests (EFForTsInformation System: University of Goettingen 2017). Datasets used in this study have the following identification numbers: 12620, 13500, 13501, 13520, 13660, 13642, 13643, 13644, 13647, 13648, 13649, 13650, 13651 (household-level data); 13521, 13600, 13601, 13620 (plot-level data); 11422, 11423, 13680 (village-level data); 11987 (soil data); 12026, 12027, 12030 (land cover maps). 


\subsection{Appendix}

Table A1: Summary statistics

\begin{tabular}{|c|c|c|c|c|c|c|}
\hline & \multicolumn{3}{|c|}{2012} & \multicolumn{3}{|c|}{2015} \\
\hline & $\begin{array}{l}\text { Number } \\
\text { of obs. }\end{array}$ & Mean & $\begin{array}{c}\text { Std. } \\
\text { deviation }\end{array}$ & $\begin{array}{c}\text { Number } \\
\text { of obs. }\end{array}$ & Mean & $\begin{array}{c}\text { Std. } \\
\text { deviation }\end{array}$ \\
\hline \multicolumn{7}{|l|}{ Plot-level variables (rubber plots) } \\
\hline Yield per year (kg/ha) & 643 & 1144.683 & 1083.612 & 466 & 1489.196 & 1086.691 \\
\hline Material input per year (000 IDR/ha) & 643 & 322.521 & 672.179 & 466 & 141.655 & 419.497 \\
\hline Labor input per year (hours/ha) & 643 & 692.655 & 630.828 & 466 & 1057.674 & 1122.030 \\
\hline Systematic land title $(=1)$ & 645 & 0.074 & & 690 & 0.100 & \\
\hline Sporadic land title $(=1)$ & 645 & 0.180 & & 690 & 0.222 & \\
\hline Plot size (ha) & 645 & 2.242 & 1.948 & 690 & 2.084 & 1.809 \\
\hline Age of rubber trees (years) & 645 & 14.763 & 10.647 & 689 & 17.026 & 10.479 \\
\hline Employing sharecropping tenants $(=1)$ & 645 & 0.143 & & 690 & 0.223 & \\
\hline Distance from household residence $(\mathrm{km})$ & 645 & 4.802 & 8.226 & 689 & 4.902 & 12.791 \\
\hline Distance from road $(\mathrm{km})$ & 645 & 1.170 & 1.757 & 689 & 0.885 & 1.410 \\
\hline \multicolumn{7}{|l|}{ Household-level variables } \\
\hline Age of household head (years) & 471 & 44.996 & 12.213 & 473 & 47.072 & 11.408 \\
\hline Female-headed household $(=1)$ & 471 & 0.059 & & 473 & 0.080 & \\
\hline Education of household head (years in school) & 471 & 7.476 & 3.620 & 473 & 7.150 & 3.742 \\
\hline Migrated to village (=1) & 471 & 0.255 & & 473 & 0.256 & \\
\hline Number of adults in household & 471 & 2.975 & 1.243 & 473 & 2.987 & 1.190 \\
\hline Total farm size (ha) & 471 & 4.200 & 4.642 & 473 & 4.134 & 4.615 \\
\hline Size of productive rubber area (ha) & 406 & 2.684 & 3.037 & 406 & 2.968 & 3.167 \\
\hline Oil palm farmer $(=1)$ & 471 & 0.285 & & 473 & 0.309 & \\
\hline Rubber farmer $(=1)$ & 471 & 0.866 & & 473 & 0.860 & \\
\hline Rubber farmer using fertilizer/pesticide $(=1)$ & 408 & 0.519 & & 406 & 0.345 & \\
\hline Oil palm farmer using fertilizer/pesticide $(=1)$ & 134 & 0.819 & & 146 & 0.818 & \\
\hline Formal credit taken $(=1)$ & 471 & 0.166 & & 473 & 0.288 & \\
\hline Informal credit taken $(=1)$ & 471 & 0.193 & & 473 & 0.173 & \\
\hline Own business $(=1)$ & 471 & 0.200 & & 473 & 0.277 & \\
\hline Share of land with systematic land title & 471 & 0.060 & & 473 & 0.089 & \\
\hline Share of land with sporadic land title & 471 & 0.167 & & 473 & 0.189 & \\
\hline Distance to market (km) & 471 & 6.940 & 7.899 & 472 & 5.616 & 5.880 \\
\hline \multicolumn{7}{|l|}{ Village-level variables } \\
\hline Number of households per village & 34 & 674.485 & 617.922 & 34 & 735.118 & 973.537 \\
\hline Share of households of Melayu ethnicity & 34 & 0.651 & 0.312 & 34 & 0.696 & 0.308 \\
\hline
\end{tabular}

Notes: In 2015, plot level input and output data were not collected for all, but only for a random sub-sample of plots. 
Table A2: Land titles and agricultural productivity

\begin{tabular}{|c|c|c|c|c|c|c|c|c|c|c|}
\hline & \multicolumn{4}{|c|}{ Household-level models } & \multicolumn{4}{|c|}{ Plot-level models } & \multicolumn{2}{|c|}{$\begin{array}{l}\text { Plot-level models with soil quality } \\
\text { controls }\end{array}$} \\
\hline & $\begin{array}{c}\text { (1) } \\
\text { Full sample } \\
\text { (RE) }\end{array}$ & $\begin{array}{c}\text { (1a) } \\
\text { Balanced } \\
\text { panel (FE) }\end{array}$ & $\begin{array}{c}\text { (2) } \\
\text { Migrants } \\
\text { (RE) }\end{array}$ & $\begin{array}{c}(2 \mathrm{a}) \\
\text { Non- } \\
\text { migrants } \\
(\mathrm{RE}) \\
\end{array}$ & $\begin{array}{c}\text { (3) } \\
\text { Full sample } \\
\text { (RE) }\end{array}$ & $\begin{array}{c}\text { (3a) } \\
\text { Balanced } \\
\text { panel (FE) }\end{array}$ & $\begin{array}{c}(4) \\
\text { Migrants } \\
\text { (RE) }\end{array}$ & $\begin{array}{c}(4 \mathrm{a}) \\
\text { Non- } \\
\text { migrants } \\
(\mathrm{RE}) \\
\end{array}$ & $\begin{array}{c}\text { (5) } \\
\text { Full sample } \\
\text { (RE) }\end{array}$ & $\begin{array}{c}\text { (6) } \\
\text { Sub-sample } \\
\text { with soil quality } \\
\text { measures (OLS) }\end{array}$ \\
\hline $\begin{array}{l}\text { Share of land with } \\
\text { systematic. title }\end{array}$ & $\begin{array}{l}0.351^{* * * *} \\
(0.085)\end{array}$ & $\begin{array}{c}0.352 \\
(0.298)\end{array}$ & $\begin{array}{l}0.586^{* * *} \\
(0.107)\end{array}$ & $\begin{array}{l}0.328^{2 * 2 *} \\
(0.098)\end{array}$ & $\begin{array}{l}0.152^{* *} \\
(0.063)\end{array}$ & $\begin{array}{c}0.025 \\
(0.269)\end{array}$ & $\begin{array}{l}0.370^{* * * *} \\
(0.098)\end{array}$ & $\begin{array}{c}0.095 \\
(0.070)\end{array}$ & $\begin{array}{l}0.182^{* * * *} \\
(0.071)\end{array}$ & $\begin{array}{l}0.697^{* *} \\
(0.265)\end{array}$ \\
\hline $\begin{array}{l}\text { Share of land with } \\
\text { sporadic title }\end{array}$ & $\begin{array}{c}0.019 \\
(0.071)\end{array}$ & $\begin{array}{l}-0.098 \\
(0.199)\end{array}$ & $\begin{array}{c}0.111 \\
(0.090)\end{array}$ & $\begin{array}{l}-0.038 \\
(0.123)\end{array}$ & $\begin{array}{l}-0.017 \\
(0.071)\end{array}$ & $\begin{array}{l}0.070 \\
(0.203)\end{array}$ & $\begin{array}{r}0.039 \\
(0.073)\end{array}$ & $\begin{array}{l}-0.070 \\
(0.106)\end{array}$ & $\begin{array}{l}-0.036 \\
(0.079)\end{array}$ & $\begin{array}{l}-0.131 \\
(0.254)\end{array}$ \\
\hline Total farm size (ha) & $\begin{array}{l}-0.025 \\
(0.022)\end{array}$ & $\begin{array}{c}0.049 \\
(0.033)\end{array}$ & $\begin{array}{l}-0.007 \\
(0.038)\end{array}$ & $\begin{array}{l}-0.020 \\
(0.026)\end{array}$ & $\begin{array}{l}-0.020^{*} \\
(0.012)\end{array}$ & $\begin{array}{l}0.014 \\
(0.029)\end{array}$ & $\begin{array}{c}0.021 \\
(0.021)\end{array}$ & $\begin{array}{l}-0.023^{*} \\
(0.013)\end{array}$ & $\begin{array}{l}-0.018 \\
(0.016)\end{array}$ & $\begin{array}{l}-0.123^{* * *} \\
(0.054)\end{array}$ \\
\hline Size of rubber area (ha) & $\begin{array}{l}-0.030^{*} \\
(0.016)\end{array}$ & $\begin{array}{l}-0.064^{* * * *} \\
(0.023)\end{array}$ & $\begin{array}{l}-0.006 \\
(0.028)\end{array}$ & $\begin{array}{l}-0.040^{* * *} \\
(0.018)\end{array}$ & $\begin{array}{l}-0.086^{* * * *} \\
(0.017)\end{array}$ & $\begin{array}{l}-0.138^{* * * *} \\
(0.049)\end{array}$ & $\begin{array}{c}-0.132^{* * *} \\
(0.029)\end{array}$ & $\begin{array}{l}-0.080^{* * * *} \\
(0.018)\end{array}$ & $\begin{array}{l}-0.088^{* * * *} \\
(0.022)\end{array}$ & $\begin{array}{l}-0.097^{*} \\
(0.049)\end{array}$ \\
\hline Wealth index (quintiles) & $\begin{array}{c}0.011 \\
(0.017)\end{array}$ & $\begin{array}{l}-0.006 \\
(0.040)\end{array}$ & $\begin{array}{l}-0.023 \\
(0.031)\end{array}$ & $\begin{array}{c}0.021 \\
(0.022)\end{array}$ & $\begin{array}{l}0.031^{* * *} \\
(0.015)\end{array}$ & $\begin{array}{c}0.015 \\
(0.038)\end{array}$ & $\begin{array}{c}0.021 \\
(0.021)\end{array}$ & $\begin{array}{l}0.042^{*} \\
(0.022)\end{array}$ & $\begin{array}{l}0.034^{*} \\
(0.019)\end{array}$ & $\begin{array}{l}0.134^{* * * *} \\
(0.049)\end{array}$ \\
\hline Number of adults & $\begin{array}{l}-0.007 \\
(0.065)\end{array}$ & $\begin{array}{c}0.036 \\
(0.044)\end{array}$ & $\begin{array}{c}0.128 \\
(0.095)\end{array}$ & $\begin{array}{l}-0.062 \\
(0.081)\end{array}$ & $\begin{array}{c}0.019 \\
(0.021)\end{array}$ & $\begin{array}{l}-0.003 \\
(0.046)\end{array}$ & $\begin{array}{l}-0.014 \\
(0.029)\end{array}$ & $\begin{array}{c}0.021 \\
(0.024)\end{array}$ & $\begin{array}{c}0.005 \\
(0.022)\end{array}$ & $\begin{array}{c}0.011 \\
(0.059)\end{array}$ \\
\hline Own business (=1) & $\begin{array}{c}0.019 \\
(0.024)\end{array}$ & $\begin{array}{c}0.033 \\
(0.099)\end{array}$ & $\begin{array}{c}0.001 \\
(0.041)\end{array}$ & $\begin{array}{c}0.024 \\
(0.029)\end{array}$ & $\begin{array}{l}-0.045 \\
(0.056)\end{array}$ & $\begin{array}{c}0.026 \\
(0.096)\end{array}$ & $\begin{array}{c}0.126 \\
(0.085)\end{array}$ & $\begin{array}{l}-0.118^{*} \\
(0.070)\end{array}$ & $\begin{array}{l}-0.026 \\
(0.065)\end{array}$ & $\begin{array}{l}-0.351^{*} \\
(0.195)\end{array}$ \\
\hline $2012(=1)$ & $\begin{array}{l}-0.075 \\
(0.049)\end{array}$ & $\begin{array}{l}-0.107^{* *} \\
(0.051)\end{array}$ & $\begin{array}{l}-0.126 \\
(0.136)\end{array}$ & $\begin{array}{l}-0.062 \\
(0.050)\end{array}$ & $\begin{array}{l}-0.114^{* *} \\
(0.046)\end{array}$ & $\begin{array}{c}-0.138^{* * * *} \\
(0.050)\end{array}$ & $\begin{array}{l}-0.080 \\
(0.100)\end{array}$ & $\begin{array}{l}-0.132^{* * * * *} \\
(0.049)\end{array}$ & $\begin{array}{l}-0.117^{* * *} \\
(0.048)\end{array}$ & \\
\hline $\begin{array}{l}\text { Age of household head } \\
\text { (years) }\end{array}$ & $\begin{array}{l}-0.001 \\
(0.003)\end{array}$ & & $\begin{array}{c}0.002 \\
(0.004)\end{array}$ & $\begin{array}{l}-0.002 \\
(0.003)\end{array}$ & $\begin{array}{l}-2 . \mathrm{E}-4 \\
(0.003)\end{array}$ & & $\begin{array}{c}0.004 \\
(0.005)\end{array}$ & $\begin{array}{l}-0.003 \\
(0.003)\end{array}$ & $\begin{array}{l}-0.001 \\
(0.003)\end{array}$ & $\begin{array}{c}-0.024^{* * *} \\
(0.009)\end{array}$ \\
\hline Female-headed household & $-0.227^{* *}$ & & $\begin{array}{l}-0.480^{* * * *} \\
(0.140)\end{array}$ & $\begin{array}{l}-0.141 \\
(0.130)\end{array}$ & $-0.196^{*}$ & & $\begin{array}{c}-0.472^{* * *} \\
(0178)\end{array}$ & $\begin{array}{l}-0.056 \\
(0.098)\end{array}$ & -0.192 & $\begin{array}{l}0.055 \\
(0.362)\end{array}$ \\
\hline $\begin{array}{l}\text { Education (years of } \\
\text { schooling) }\end{array}$ & $\begin{array}{c}0.010 \\
(0.010)\end{array}$ & & $\begin{array}{c}0.004 \\
(0.012)\end{array}$ & $\begin{array}{c}0.012 \\
(0.011)\end{array}$ & $\begin{array}{l}0.017^{*} \\
(0.009)\end{array}$ & & $\begin{array}{l}0.008 \\
(0.011)\end{array}$ & $\begin{array}{l}0.021^{* * *} \\
(0.010)\end{array}$ & $\begin{array}{l}0.016^{*} \\
(0.010)\end{array}$ & $\begin{array}{l}0.015 \\
(0.020)\end{array}$ \\
\hline Farm size squared (ha) & $\begin{array}{l}0.001^{* * *} \\
(0.001)\end{array}$ & & $\begin{array}{l}\text { 4.E-4 } \\
(0.001)\end{array}$ & $\begin{array}{l}0.001^{* *} \\
(0.001)\end{array}$ & $\begin{array}{l}0.001^{* * *} \\
(4 . E-4)\end{array}$ & & $\begin{array}{c}\text { 7.E-5 } \\
(0.001)\end{array}$ & $\begin{array}{l}0.001^{* * *} \\
(5 . E-4)\end{array}$ & $\begin{array}{l}0.001^{* *} \\
(0.001)\end{array}$ & $\begin{array}{l}0.008^{* * *} \\
(0.003)\end{array}$ \\
\hline Non-random village $(=1)$ & $\begin{array}{l}-0.165^{* *} \\
(0.068)\end{array}$ & & $\begin{array}{c}-0.215^{* * * *} \\
(0.072)\end{array}$ & $\begin{array}{l}-0.126 \\
(0.078)\end{array}$ & $\begin{array}{l}-0.191^{* * *} \\
(0.067)\end{array}$ & & $\begin{array}{l}-0.227^{* *} \\
(0.099)\end{array}$ & $\begin{array}{l}-0.137^{* * *} \\
(0.056)\end{array}$ & $\begin{array}{l}-0.167^{* *} \\
(0.066)\end{array}$ & \\
\hline Migrant (=1) & $\begin{array}{c}0.056 \\
(0.065)\end{array}$ & & & & $\begin{array}{c}0.040 \\
(0.066)\end{array}$ & & & & $\begin{array}{c}0.019 \\
(0.067)\end{array}$ & $\begin{array}{l}-0.115 \\
(0.200)\end{array}$ \\
\hline $\begin{array}{l}\text { Age of rubber trees } \\
\text { (years) }\end{array}$ & & & & & $\begin{array}{l}0.017^{*} \\
(0.009)\end{array}$ & & $\begin{array}{c}0.028 \\
(0.017)\end{array}$ & $\begin{array}{c}0.013 \\
(0.010)\end{array}$ & $\begin{array}{l}0.023^{* *} \\
(0.009)\end{array}$ & $\begin{array}{l}-0.004 \\
(0.038)\end{array}$ \\
\hline $\begin{array}{l}\text { Age of trees (years } \\
\text { squared) }\end{array}$ & & & & & $\begin{array}{l}-4 . E-4^{*} \\
(2 . E-4)\end{array}$ & & $\begin{array}{l}-0.001 \\
(4 . E-4)\end{array}$ & $\begin{array}{l}-3 . \mathrm{E}-4^{*} \\
(2 . \mathrm{E}-4)\end{array}$ & $\begin{array}{l}-0.001^{* * *} \\
(2 . \mathrm{E}-4)\end{array}$ & $\begin{array}{l}1 . \mathrm{E}-06 \\
(0.001)\end{array}$ \\
\hline Employing sharecroppers & & & & & $\begin{array}{l}0.118^{*} \\
(0.064)\end{array}$ & & $\begin{array}{c}0.171 \\
(0.110)\end{array}$ & $\begin{array}{c}0.098 \\
(0.069)\end{array}$ & $\begin{array}{c}0.077 \\
(0.062)\end{array}$ & $\begin{array}{l}0.360^{* *} \\
(0.178)\end{array}$ \\
\hline $\begin{array}{l}\text { Distance from residence } \\
(\mathrm{km})\end{array}$ & & & & & $\begin{array}{l}-0.002 \\
(0.003)\end{array}$ & & $\begin{array}{c}-0.025^{* * *} \\
(0.008)\end{array}$ & $\begin{array}{c}0.003 \\
(0.004)\end{array}$ & $\begin{array}{l}-0.003 \\
(0.006)\end{array}$ & $\begin{array}{l}-0.012 \\
(0.013)\end{array}$ \\
\hline Distance from road $(\mathrm{km})$ & & & & & $\begin{array}{l}0.005 \\
(0.016)\end{array}$ & & $\begin{array}{l}0.055^{* *} \\
(0.026)\end{array}$ & $\begin{array}{l}-0.007 \\
(0.019)\end{array}$ & $\begin{array}{c}0.009 \\
(0.017)\end{array}$ & $\begin{array}{c}0.013 \\
(0.066)\end{array}$ \\
\hline
\end{tabular}




\begin{tabular}{|c|c|c|c|c|c|c|c|c|c|c|}
\hline & \multicolumn{4}{|c|}{ Household-level models } & \multicolumn{4}{|c|}{ Plot-level models } & \multicolumn{2}{|c|}{$\begin{array}{c}\text { Plot-level models with soil quality } \\
\text { controls }\end{array}$} \\
\hline & $\begin{array}{c}(1) \\
\text { Full sample } \\
\text { (RE) }\end{array}$ & $\begin{array}{c}\text { (1a) } \\
\text { Balanced } \\
\text { panel (FE) }\end{array}$ & $\begin{array}{c}(2) \\
\text { Migrants } \\
\text { (RE) }\end{array}$ & $\begin{array}{l}(2 \mathrm{a}) \\
\text { Non- } \\
\text { migrants } \\
(\mathrm{RE})\end{array}$ & $\begin{array}{c}(3) \\
\text { Full sample } \\
\text { (RE) }\end{array}$ & $\begin{array}{c}\text { (3a) } \\
\text { Balanced } \\
\text { panel (FE) }\end{array}$ & $\begin{array}{c}\text { (4) } \\
\text { Migrants } \\
\text { (RE) }\end{array}$ & $\begin{array}{l}(4 \mathrm{a}) \\
\text { Non- } \\
\text { migrants } \\
(\mathrm{RE})\end{array}$ & $\begin{array}{c}(5) \\
\text { Full sample } \\
\text { (RE) }\end{array}$ & $\begin{array}{c}(6) \\
\text { Sub-sample with } \\
\text { soil quality } \\
\text { measures (OLS) }\end{array}$ \\
\hline Altitude of residence (m) & & & & & & & & & $\begin{array}{l}-2 . \mathrm{E}-4 \\
(0.001)\end{array}$ & \\
\hline Medium soil fertility (=1) & & & & & & & & & 0.012 & \\
\hline High soil fertility $(=1)$ & & & & & & & & & -0.042 & \\
\hline (Ref. =low fertility) & & & & & & & & & $(0.112)$ & \\
\hline Soil bulk density & & & & & & & & & & $\begin{array}{l}-0.600 \\
(0.456)\end{array}$ \\
\hline Soil carbon content & & & & & & & & & & $\begin{array}{l}-0.088 \\
(0.099)\end{array}$ \\
\hline Carbon content (squared) & & & & & & & & & & $\begin{array}{c}0.003 \\
(0.003)\end{array}$ \\
\hline Carbon/nitrogen ratio & & & & & & & & & & $\begin{array}{c}0.032 \\
(0.037)\end{array}$ \\
\hline Constant & $\begin{array}{l}7.233^{* * * *} \\
(0.150)\end{array}$ & $\begin{array}{l}7.105^{* * *} \\
(0.211)\end{array}$ & $\begin{array}{l}7.188^{* * * *} \\
(0.244)\end{array}$ & $\begin{array}{l}7.279^{* * * *} \\
(0.171)\end{array}$ & $\begin{array}{l}7.126^{* * *} \\
(0.199)\end{array}$ & $\begin{array}{l}7.494^{* * * *} \\
(0.234)\end{array}$ & $\begin{array}{l}6.967^{* * *} \\
(0.347)\end{array}$ & $\begin{array}{l}7.220^{* * *} \\
(0.237)\end{array}$ & $\begin{array}{l}7.163^{* * * *} \\
(0.256)\end{array}$ & $\begin{array}{l}8.760^{* * * *} \\
(0.852)\end{array}$ \\
\hline $\begin{array}{l}\text { Chi2 / F-statistic } \\
\text { Hausman test (chi2) }\end{array}$ & $297.453^{* * *}$ & $\begin{array}{l}1.986^{* * *} \\
2.24\end{array}$ & $232.371^{* * * *}$ & $123.891^{* * *}$ & $312.312^{* * * *}$ & $\begin{array}{l}1.89^{* *} \\
4.67\end{array}$ & $2332.550^{* * * *}$ & $550.142^{* * *}$ & $482.379^{* * *}$ & $3.634^{* * * *}$ \\
\hline Number of observations & 665 & 564 & 174 & 491 & 851 & 516 & 231 & 620 & 741 & 92 \\
\hline
\end{tabular}


Table A3: Historical forest coverage and land titles (spatial error and spatial lag models)

\begin{tabular}{|c|c|c|c|c|c|c|c|c|}
\hline & \multicolumn{4}{|c|}{ Spatial error models } & \multicolumn{4}{|c|}{ Spatial lag models } \\
\hline & (1) & (2) & (3) & (4) & (5) & (6) & (7) & (8) \\
\hline & $\begin{array}{l}2 \mathrm{~km} \\
\text { radius }\end{array}$ & $\begin{array}{l}5 \mathrm{~km} \\
\text { radius }\end{array}$ & $\begin{array}{l}10 \mathrm{~km} \\
\text { radius }\end{array}$ & All plots & $\begin{array}{c}2 \mathrm{~km} \\
\text { radius }\end{array}$ & $\begin{array}{l}5 \mathrm{~km} \\
\text { radius }\end{array}$ & $\begin{array}{l}10 \mathrm{~km} \\
\text { radius }\end{array}$ & All plots \\
\hline $\begin{array}{l}\text { Share of forested } \\
\text { area in } 1990\end{array}$ & $\begin{array}{c}-0.160^{* * *} \\
(0.050)\end{array}$ & $\begin{array}{l}-0.113^{* *} \\
(0.048)\end{array}$ & $\begin{array}{c}-0.150^{* * *} \\
(0.057)\end{array}$ & & $\begin{array}{c}-0.164^{* * *} \\
(0.053)\end{array}$ & $\begin{array}{c}-0.107^{* * *} \\
(0.046)\end{array}$ & $\begin{array}{c}-0.146^{* * *} \\
(0.056)\end{array}$ & \\
\hline $\begin{array}{l}\text { Deforestation } \\
(=1)\end{array}$ & & & & $\begin{array}{l}-0.068^{* *} \\
(0.027)\end{array}$ & & & & $\begin{array}{c}-0.068^{* *} \\
(0.027)\end{array}$ \\
\hline Rubber plot $(=1)$ & $\begin{array}{l}-0.056 \\
(0.042)\end{array}$ & $\begin{array}{c}-0.096^{* * * *} \\
(0.034)\end{array}$ & $\begin{array}{c}-0.078^{* * *} \\
(0.031)\end{array}$ & $\begin{array}{c}-0.105^{* * * *} \\
(0.032)\end{array}$ & $\begin{array}{l}-0.057 \\
(0.042)\end{array}$ & $\begin{array}{c}-0.095^{* * *} \\
(0.033)\end{array}$ & $\begin{array}{c}-0.077^{* * *} \\
(0.031)\end{array}$ & $\begin{array}{c}-0.105^{* * * *} \\
(0.031)\end{array}$ \\
\hline $\begin{array}{l}\text { Duration of plot } \\
\text { ownership (years) }\end{array}$ & $\begin{array}{c}0.007^{* * * *} \\
(0.002)\end{array}$ & $\begin{array}{c}0.006^{* * * *} \\
(0.002)\end{array}$ & $\begin{array}{c}0.005^{* * * *} \\
(0.001)\end{array}$ & $\begin{array}{c}0.006^{* * *} \\
(0.001)\end{array}$ & $\begin{array}{c}0.007^{* * * *} \\
(0.002)\end{array}$ & $\begin{array}{c}0.006^{* * * *} \\
(0.002)\end{array}$ & $\begin{array}{c}0.005^{* * *} \\
(0.001)\end{array}$ & $\begin{array}{c}0.006^{* * * *} \\
(0.001)\end{array}$ \\
\hline $\begin{array}{l}\text { Distance from } \\
\text { road }(\mathrm{km})\end{array}$ & $\begin{array}{c}-0.086^{* * * *} \\
(0.030)\end{array}$ & $\begin{array}{c}-0.041^{* * * *} \\
(0.013)\end{array}$ & $\begin{array}{c}-0.025^{* *} \\
(0.010)\end{array}$ & $\begin{array}{l}-0.020^{* * *} \\
(0.008)\end{array}$ & $\begin{array}{c}-0.085^{* * *} \\
(0.030)\end{array}$ & $\begin{array}{c}-0.042^{* * * *} \\
(0.013)\end{array}$ & $\begin{array}{c}-0.025^{* * *} \\
(0.010)\end{array}$ & $\begin{array}{c}-0.021^{* * *} \\
(0.008)\end{array}$ \\
\hline $\begin{array}{l}\text { Age of household } \\
\text { head (years) }\end{array}$ & $\begin{array}{l}-0.002 \\
(0.002)\end{array}$ & $\begin{array}{l}-0.001 \\
(0.001)\end{array}$ & $\begin{array}{l}-0.001 \\
(0.001)\end{array}$ & $\begin{array}{l}-3 . \mathrm{E}-4 \\
(0.001)\end{array}$ & $\begin{array}{l}-0.002 \\
(0.002)\end{array}$ & $\begin{array}{l}-0.001 \\
(0.001)\end{array}$ & $\begin{array}{l}-0.001 \\
(0.001)\end{array}$ & $\begin{array}{l}-3 . \mathrm{E}-4 \\
(0.001)\end{array}$ \\
\hline $\begin{array}{l}\text { Education (years } \\
\text { of schooling) }\end{array}$ & $\begin{array}{l}0.005 \\
(0.005)\end{array}$ & $\begin{array}{l}4 . \mathrm{C}-4 \\
(0.004)\end{array}$ & $\begin{array}{c}0.001 \\
(0.003)\end{array}$ & $\begin{array}{l}4 . \mathrm{E}-4 \\
(0.004)\end{array}$ & $\begin{array}{c}0.004 \\
(0.005)\end{array}$ & $\begin{array}{l}4 . \mathrm{E}-4 \\
(0.004)\end{array}$ & $\begin{array}{c}0.001 \\
(0.003)\end{array}$ & $\begin{array}{l}0.001 \\
(0.004)\end{array}$ \\
\hline Migrant (=1) & $\begin{array}{l}-0.028 \\
(0.042)\end{array}$ & $\begin{array}{l}-0.013 \\
(0.034)\end{array}$ & $\begin{array}{c}0.012 \\
(0.031)\end{array}$ & $\begin{array}{l}-0.014 \\
(0.030)\end{array}$ & $\begin{array}{l}-0.030 \\
(0.042)\end{array}$ & $\begin{array}{l}-0.013 \\
(0.034)\end{array}$ & $\begin{array}{c}0.012 \\
(0.031)\end{array}$ & $\begin{array}{l}-0.013 \\
(0.030)\end{array}$ \\
\hline $\begin{array}{l}\text { Wealth index } \\
\text { (initial, quintiles) }\end{array}$ & $\begin{array}{l}0.008 \\
(0.015)\end{array}$ & $\begin{array}{c}0.012 \\
(0.012)\end{array}$ & $\begin{array}{c}0.017 \\
(0.011)\end{array}$ & $\begin{array}{c}0.028^{* * * *} \\
(0.011)\end{array}$ & $\begin{array}{c}0.007 \\
(0.015)\end{array}$ & $\begin{array}{c}0.012 \\
(0.012)\end{array}$ & $\begin{array}{l}0.018^{*} \\
(0.011)\end{array}$ & $\begin{array}{c}0.029^{* * * *} \\
(0.011)\end{array}$ \\
\hline $\begin{array}{l}\text { Total farm size } \\
\text { (ha) }\end{array}$ & $\begin{array}{l}-0.001 \\
(0.003)\end{array}$ & $\begin{array}{l}-0.003 \\
(0.002)\end{array}$ & $\begin{array}{l}-0.002 \\
(0.002)\end{array}$ & $\begin{array}{l}-0.004^{*} \\
(0.002)\end{array}$ & $\begin{array}{l}-0.001 \\
(0.003)\end{array}$ & $\begin{array}{l}-0.003 \\
(0.002)\end{array}$ & $\begin{array}{l}-0.002 \\
(0.002)\end{array}$ & $\begin{array}{l}-0.004^{*} \\
(0.002)\end{array}$ \\
\hline $\begin{array}{l}\text { Non-random } \\
\text { village }(=1)\end{array}$ & $\begin{array}{l}-0.066 \\
(0.052)\end{array}$ & $\begin{array}{l}-0.056 \\
(0.043)\end{array}$ & $\begin{array}{l}-0.007 \\
(0.040)\end{array}$ & $\begin{array}{l}-0.013 \\
(0.042)\end{array}$ & $\begin{array}{l}-0.066 \\
(0.055)\end{array}$ & $\begin{array}{l}-0.055 \\
(0.042)\end{array}$ & $\begin{array}{l}-0.007 \\
(0.039)\end{array}$ & $\begin{array}{l}-0.015 \\
(0.039)\end{array}$ \\
\hline $\begin{array}{l}\text { village }(=1) \\
\text { Share of migrants }\end{array}$ & $0.195^{* *}$ & $0.190^{* * * *}$ & $0.126^{* * *}$ & $0.128^{* * *}$ & $0.202^{* *}$ & $0.183^{* * *}$ & $0.123^{* * *}$ & $\begin{array}{l}(0.039) \\
0.117^{*}\end{array}$ \\
\hline in village & $(0.078)$ & $(0.070)$ & $(0.060)$ & $(0.065)$ & $(0.083)$ & $(0.068)$ & $(0.059)$ & (0.061) \\
\hline $\begin{array}{l}\text { Village wealth } \\
\text { index (initial, } \\
\text { quintiles) }\end{array}$ & $\begin{array}{l}-0.025^{*} \\
(0.014)\end{array}$ & $\begin{array}{l}-0.011 \\
(0.012)\end{array}$ & $\begin{array}{l}-0.005 \\
(0.010)\end{array}$ & $\begin{array}{l}-0.003 \\
(0.011)\end{array}$ & $\begin{array}{l}-0.026 \\
(0.015)\end{array}$ & $\begin{array}{l}-0.011 \\
(0.011)\end{array}$ & $\begin{array}{l}-0.005 \\
(0.010)\end{array}$ & $\begin{array}{l}-0.004 \\
(0.011)\end{array}$ \\
\hline Constant & $\begin{array}{l}0.276^{* *} \\
(0.132)\end{array}$ & $\begin{array}{c}0.297^{* * * *} \\
(0.110)\end{array}$ & $\begin{array}{l}0.198^{*} \\
(0.102)\end{array}$ & $\begin{array}{c}0.141 \\
(0.110)\end{array}$ & $\begin{array}{l}0.287^{* *} \\
(0.134)\end{array}$ & $\begin{array}{c}0.287^{* * * *} \\
(0.109)\end{array}$ & $\begin{array}{l}0.191^{*} \\
(0.101)\end{array}$ & $\begin{array}{c}0.135 \\
(0.107)\end{array}$ \\
\hline $\begin{array}{l}\text { Regency } \\
\text { dummies } \\
\text { included }\end{array}$ & Yes & Yes & Yes & Yes & Yes & Yes & Yes & Yes \\
\hline $\begin{array}{l}\text { Lambda and rho } \\
\text { Constant }\end{array}$ & $\begin{array}{l}-0.079 \\
(0.104)\end{array}$ & $\begin{array}{c}0.062 \\
(0.095)\end{array}$ & $\begin{array}{c}0.039 \\
(0.091)\end{array}$ & $\begin{array}{c}0.122 \\
(0.103)\end{array}$ & $\begin{array}{l}-0.050 \\
(0.099)\end{array}$ & $\begin{array}{c}0.068 \\
(0.092)\end{array}$ & $\begin{array}{c}0.032 \\
(0.087)\end{array}$ & $\begin{array}{c}0.148 \\
(0.096)\end{array}$ \\
\hline $\begin{array}{l}\text { Squared } \\
\text { correlation }\end{array}$ & 0.088 & 0.074 & 0.057 & 0.099 & 0.089 & 0.075 & 0.058 & 0.104 \\
\hline Number of obs. & 405 & 620 & 734 & 573 & 405 & 620 & 734 & 573 \\
\hline
\end{tabular}


Table A4: Historical forest coverage and farm size (spatial error and OLS models)

\begin{tabular}{|c|c|c|c|c|c|c|c|c|}
\hline & \multicolumn{4}{|c|}{ Spatial error model } & \multicolumn{4}{|c|}{ Ordinary least squares } \\
\hline & $\begin{array}{c}(1) \\
2 \mathrm{~km} \\
\text { radius }\end{array}$ & $\begin{array}{c}(2) \\
5 \mathrm{~km} \\
\text { radius }\end{array}$ & $\begin{array}{c}(3) \\
10 \mathrm{~km} \\
\text { radius }\end{array}$ & $\begin{array}{c}\text { (4) } \\
\text { All plots }\end{array}$ & $\begin{array}{c}(5) \\
2 \mathrm{~km} \\
\text { radius }\end{array}$ & $\begin{array}{c}(6) \\
5 \mathrm{~km} \\
\text { radius }\end{array}$ & $\begin{array}{c}(7) \\
10 \mathrm{~km} \\
\text { radius }\end{array}$ & $\begin{array}{c}\text { (8) } \\
\text { All plots }\end{array}$ \\
\hline $\begin{array}{l}\text { Share of forested } \\
\text { area in } 1990\end{array}$ & $\begin{array}{l}0.325^{*} \\
(0.178)\end{array}$ & $\begin{array}{l}0.406^{* *} \\
(0.187)\end{array}$ & $\begin{array}{l}0.535^{* *} \\
(0.240)\end{array}$ & & $\begin{array}{l}0.321^{*} \\
(0.164)\end{array}$ & $\begin{array}{l}0.412^{* *} \\
(0.177)\end{array}$ & $\begin{array}{l}0.551^{* *} \\
(0.233)\end{array}$ & \\
\hline Deforestation $(=1)$ & & & & $\begin{array}{l}0.236^{* * *} \\
(0.089)\end{array}$ & & & & $\begin{array}{l}0.283^{* * * *} \\
(0.086)\end{array}$ \\
\hline $\begin{array}{l}\text { Age of household } \\
\text { head (years) }\end{array}$ & $\begin{array}{l}0.012^{* * *} \\
(0.005)\end{array}$ & $\begin{array}{l}0.012^{* *} \\
(0.005)\end{array}$ & $\begin{array}{l}0.012^{* *} \\
(0.005)\end{array}$ & $\begin{array}{l}0.009^{*} \\
(0.005)\end{array}$ & $\begin{array}{l}0.011^{* * *} \\
(0.004)\end{array}$ & $\begin{array}{l}0.011^{* * * *} \\
(0.004)\end{array}$ & $\begin{array}{l}0.011^{* * *} \\
(0.004)\end{array}$ & $\begin{array}{l}0.008^{* *} \\
(0.004)\end{array}$ \\
\hline $\begin{array}{l}\text { Education (years of } \\
\text { schooling) }\end{array}$ & $\begin{array}{l}0.038^{* * * *} \\
(0.012)\end{array}$ & $\begin{array}{l}0.038^{* * *} \\
(0.012)\end{array}$ & $\begin{array}{l}0.038^{* * *} \\
(0.012)\end{array}$ & $\begin{array}{l}0.038^{* * *} \\
(0.012)\end{array}$ & $\begin{array}{l}0.038^{* *} \\
(0.014)\end{array}$ & $\begin{array}{l}0.037^{* *} \\
(0.014)\end{array}$ & $\begin{array}{l}0.037^{* *} \\
(0.014)\end{array}$ & $\begin{array}{l}0.039^{* * *} \\
(0.013)\end{array}$ \\
\hline Migrant (=1) & $\begin{array}{c}0.112 \\
(0.106)\end{array}$ & $\begin{array}{c}0.112 \\
(0.106)\end{array}$ & $\begin{array}{c}0.110 \\
(0.106)\end{array}$ & $\begin{array}{c}0.128 \\
(0.105)\end{array}$ & $\begin{array}{c}0.155 \\
(0.133)\end{array}$ & $\begin{array}{c}0.152 \\
(0.131)\end{array}$ & $\begin{array}{c}0.149 \\
(0.130)\end{array}$ & $\begin{array}{c}0.169 \\
(0.131)\end{array}$ \\
\hline $\begin{array}{l}\text { Age of household } \\
\text { (years) }\end{array}$ & $\begin{array}{l}0.009^{*} \\
(0.005)\end{array}$ & $\begin{array}{l}0.009^{*} \\
(0.005)\end{array}$ & $\begin{array}{l}0.008^{*} \\
(0.005)\end{array}$ & $\begin{array}{l}0.008^{*} \\
(0.005)\end{array}$ & $\begin{array}{l}0.009^{* *} \\
(0.004)\end{array}$ & $\begin{array}{l}0.009^{* *} \\
(0.004)\end{array}$ & $\begin{array}{l}0.009^{* *} \\
(0.004)\end{array}$ & $\begin{array}{l}0.008^{* *} \\
(0.004)\end{array}$ \\
\hline $\begin{array}{l}\text { Wealth index (initial, } \\
\text { quintiles) }\end{array}$ & $\begin{array}{l}0.142^{* * *} \\
(0.035)\end{array}$ & $\begin{array}{l}0.144^{* * * *} \\
(0.035)\end{array}$ & $\begin{array}{l}0.146^{* * * *} \\
(0.035)\end{array}$ & $\begin{array}{l}0.141^{* * * *} \\
(0.035)\end{array}$ & $\begin{array}{l}0.144^{* * * *} \\
(0.036)\end{array}$ & $\begin{array}{l}0.147^{* * * *} \\
(0.036)\end{array}$ & $\begin{array}{c}0.149^{* * *} \\
(0.037)\end{array}$ & $\begin{array}{l}0.142^{* * * *} \\
(0.036)\end{array}$ \\
\hline $\begin{array}{l}\text { Share of migrants in } \\
\text { village }\end{array}$ & $\begin{array}{l}0.757^{* * * *} \\
(0.227)\end{array}$ & $\begin{array}{l}0.763^{* * *} \\
(0.225)\end{array}$ & $\begin{array}{l}0.751^{* * *} \\
(0.224)\end{array}$ & $\begin{array}{l}0.733^{* * *} \\
(0.221)\end{array}$ & $\begin{array}{l}0.701^{* *} \\
(0.299)\end{array}$ & $\begin{array}{l}0.710^{* *} \\
(0.295)\end{array}$ & $\begin{array}{l}0.702^{* *} \\
(0.294)\end{array}$ & $\begin{array}{l}0.691^{* *} \\
(0.278)\end{array}$ \\
\hline $\begin{array}{l}\text { Village wealth index } \\
\text { (initial, quintiles) }\end{array}$ & $\begin{array}{c}-0.122^{* * *} \\
(0.039)\end{array}$ & $\begin{array}{c}-0.124^{* * * *} \\
(0.039)\end{array}$ & $\begin{array}{c}-0.129^{* * * *} \\
(0.039)\end{array}$ & $\begin{array}{c}-0.121^{* * * *} \\
(0.038)\end{array}$ & $\begin{array}{c}-0.127^{* * * *} \\
(0.038)\end{array}$ & $\begin{array}{c}-0.129^{* * *} \\
(0.038)\end{array}$ & $\begin{array}{c}-0.134^{* * * *} \\
(0.038)\end{array}$ & $\begin{array}{c}-0.126^{* * * *} \\
(0.037)\end{array}$ \\
\hline $\begin{array}{l}\text { Non-random village } \\
(=1)\end{array}$ & $\begin{array}{c}0.214 \\
(0.163)\end{array}$ & $\begin{array}{c}0.183 \\
(0.161)\end{array}$ & $\begin{array}{c}0.137 \\
(0.162)\end{array}$ & $\begin{array}{c}0.212 \\
(0.158)\end{array}$ & $\begin{array}{l}0.218^{*} \\
(0.128)\end{array}$ & $\begin{array}{c}0.186 \\
(0.130)\end{array}$ & $\begin{array}{c}0.140 \\
(0.139)\end{array}$ & $\begin{array}{l}0.224^{*} \\
(0.119)\end{array}$ \\
\hline Constant & $\begin{array}{l}-0.307 \\
(0.312)\end{array}$ & $\begin{array}{l}-0.325 \\
(0.310)\end{array}$ & $\begin{array}{l}-0.354 \\
(0.311)\end{array}$ & $\begin{array}{l}-0.246 \\
(0.304)\end{array}$ & $\begin{array}{l}-0.237 \\
(0.317)\end{array}$ & $\begin{array}{l}-0.262 \\
(0.319)\end{array}$ & $\begin{array}{l}-0.298 \\
(0.326)\end{array}$ & $\begin{array}{l}-0.204 \\
(0.291)\end{array}$ \\
\hline $\begin{array}{l}\text { Regency dummies } \\
\text { included }\end{array}$ & Yes & Yes & Yes & Yes & Yes & Yes & Yes & Yes \\
\hline $\begin{array}{l}\text { Lambda } \\
\text { Constant }\end{array}$ & $\begin{array}{l}0.253^{* * *} \\
(0.084)\end{array}$ & $\begin{array}{l}0.243^{* * * *} \\
(0.084)\end{array}$ & $\begin{array}{l}0.240^{* * *} \\
(0.084)\end{array}$ & $\begin{array}{l}0.226^{* * *} \\
(0.086)\end{array}$ & & & & \\
\hline $\begin{array}{l}\text { F-stat./squared corr. } \\
\text { Number of obs. }\end{array}$ & $\begin{array}{c}0.186 \\
462 \\
\end{array}$ & $\begin{array}{c}0.190 \\
462 \\
\end{array}$ & $\begin{array}{c}0.191 \\
462 \\
\end{array}$ & $\begin{array}{c}0.196 \\
462 \\
\end{array}$ & $\begin{array}{c}8.354 \\
462 \\
\end{array}$ & $\begin{array}{c}8.589 \\
462 \\
\end{array}$ & $\begin{array}{c}8.621 \\
462 \\
\end{array}$ & $\begin{array}{c}8.152 \\
462 \\
\end{array}$ \\
\hline
\end{tabular}

Notes: The dependent variable in all models is log of farm size in 2015 measured in hectares. Coefficient estimates are shown with standard errors in parentheses (clustered at village level in columns 5-8). Goodness of fit measure for the spatial error models is the squared correlation. Lambda is the coefficient of spatially autocorrelated errors. ${ }^{*} p \leq 0.10,{ }^{* * *} p$ $\leq 0.05,{ }^{* * * *} p \leq 0.01$. 
Table A5: Land titles and agricultural intensity

\begin{tabular}{|c|c|c|c|c|c|c|}
\hline & \multicolumn{6}{|c|}{ Plot-level models } \\
\hline & \multirow{5}{*}{$\begin{array}{c}(1) \\
\text { Material input } \\
\text { (000 IDR/ha) } \\
\text { Full sample }\end{array}$} & \multirow{5}{*}{$\begin{array}{c}(2) \\
\text { Material input } \\
\text { (000 IDR/ha) } \\
\text { Migrants }\end{array}$} & \multicolumn{2}{|r|}{ (4) } & \multirow{5}{*}{$\begin{array}{c}\text { (5) } \\
\text { Log of yield } \\
(\mathrm{kg} / \mathrm{ha}) \\
\text { Full sample }\end{array}$} & \multirow{5}{*}{$\begin{array}{c}(6) \\
\begin{array}{c}\text { Log of yield } \\
(\mathrm{kg} / \mathrm{ha})\end{array} \\
\text { Full sample }\end{array}$} \\
\hline & & & Log of labor & Log of labor & & \\
\hline & & & input & input & & \\
\hline & & & (hours/ha) & (hours/ha) & & \\
\hline & & & Full sample & Migrants & & \\
\hline \multirow{4}{*}{$\begin{array}{l}\text { Systematic land title } \\
(=1) \\
\text { Sporadic land title }(=1)\end{array}$} & $114.148^{* * *}$ & $204.127^{* * 3}$ & $0.125^{*}$ & 0.122 & $0.141^{* * *}$ & $0.145^{* *}$ \\
\hline & $(48.649)$ & $(97.340)$ & $(0.070)$ & $(0.104)$ & $(0.062)$ & $(0.062)$ \\
\hline & -9.365 & 26.157 & 0.055 & $0.198^{*}$ & -0.015 & -0.026 \\
\hline & $(36.395)$ & $(61.016)$ & $(0.056)$ & $(0.105)$ & $(0.073)$ & $(0.062)$ \\
\hline \multirow[t]{2}{*}{ Total farm size (ha) } & $14.887^{* *}$ & 23.495 & $-0.035^{*}$ & 0.006 & $-0.022^{* *}$ & -0.002 \\
\hline & $(7.195)$ & $(17.456)$ & $(0.018)$ & $(0.035)$ & $(0.011)$ & $(0.010)$ \\
\hline \multirow[t]{2}{*}{ Farm size squared (ha) } & -0.241 & -0.285 & $0.001^{*}$ & -0.001 & $0.001^{* * *}$ & $0.001^{* *}$ \\
\hline & $(0.231)$ & $(0.659)$ & $(0.001)$ & $(0.001)$ & (4.E-4) & (3.E-4) \\
\hline \multirow{2}{*}{$\begin{array}{l}\text { Wealth index } \\
\text { (quintiles) }\end{array}$} & $38.959^{* * * *}$ & 9.467 & -0.007 & -0.011 & $0.029^{*}$ & $0.027^{*}$ \\
\hline & (11.018) & (22.878) & $(0.023)$ & $(0.042)$ & $(0.015)$ & $(0.014)$ \\
\hline \multirow[t]{2}{*}{ Own business $(=1)$} & -11.332 & 102.631 & 0.023 & 0.073 & -0.047 & -0.068 \\
\hline & $(33.826)$ & $(65.527)$ & $(0.056)$ & $(0.117)$ & $(0.054)$ & $(0.057)$ \\
\hline \multirow[t]{2}{*}{ Number of adults } & -4.377 & -0.566 & 0.031 & 0.045 & 0.019 & 0.007 \\
\hline & $(12.091)$ & $(27.882)$ & $(0.028)$ & $(0.053)$ & $(0.020)$ & $(0.018)$ \\
\hline \multirow{2}{*}{$\begin{array}{l}\text { Age of household head } \\
\text { (years) }\end{array}$} & -0.414 & 1.233 & 0.003 & 0.004 & $-2 . \mathrm{E}-4$ & -0.002 \\
\hline & $(1.451)$ & (3.207) & $(0.004)$ & $(0.007)$ & $(0.003)$ & $(0.002)$ \\
\hline \multirow{2}{*}{$\begin{array}{l}\text { Female-headed } \\
\text { household }(=1)\end{array}$} & $-181.132^{* *}$ & -324.205 & -0.246 & $-0.707^{*}$ & $-0.186^{*}$ & -0.068 \\
\hline & $(76.972)$ & $(225.738)$ & $(0.197)$ & $(0.406)$ & $(0.103)$ & $(0.092)$ \\
\hline \multirow{2}{*}{$\begin{array}{l}\text { Education (years of } \\
\text { schooling) }\end{array}$} & 2.652 & $26.751^{* * *}$ & -0.011 & -0.022 & $0.017^{*}$ & $0.020^{* * *}$ \\
\hline & $(4.375)$ & $(9.153)$ & $(0.010)$ & $(0.021)$ & $(0.009)$ & $(0.009)$ \\
\hline \multirow[t]{2}{*}{ Migrant (=1) } & $113.687^{* * * *}$ & & -0.061 & & 0.027 & 0.060 \\
\hline & (32.706) & & $(0.056)$ & & $(0.067)$ & $(0.064)$ \\
\hline \multirow[t]{2}{*}{ Plot size (ha) } & -7.491 & -14.137 & $-0.104^{* * *}$ & -0.063 & $-0.084^{* * *}$ & $-0.053^{* * *}$ \\
\hline & $(9.024)$ & (21.056) & $(0.021)$ & $(0.038)$ & $(0.017)$ & $(0.014)$ \\
\hline \multirow{2}{*}{$\begin{array}{l}\text { Age of rubber trees } \\
\text { (years) }\end{array}$} & $-32.052^{* * * *}$ & $-33.473^{* * *}$ & -0.014 & -0.028 & $0.020^{* * *}$ & 0.011 \\
\hline & $(5.586)$ & $(10.745)$ & (0.009) & $(0.018)$ & $(0.010)$ & (0.009) \\
\hline Age of trees (years & $0.542^{* * * *}$ & $0.560^{* *}$ & 3.E-4 & $6 . \mathrm{E}-4$ & $-4 . \mathrm{E}-4$ & $-2 . \mathrm{E}-4$ \\
\hline squared) & $(0.123)$ & $(0.235)$ & (2.E-4) & (4.E-4) & (2.E-4) & $(2 . E-4)$ \\
\hline Plot productive $(=1)$ & $191.446^{* * * *}$ & $392.696^{* * * *}$ & $3.393^{* * * *}$ & $3.417^{* * * *}$ & & \\
\hline & $(45.729)$ & $(91.030)$ & $(0.127)$ & $(0.211)$ & & \\
\hline Employing & -62.861 & -45.360 & -0.077 & -0.202 & $0.121^{* * *}$ & $0.136^{* *}$ \\
\hline sharecroppers $(=1)$ & $(42.254)$ & $(82.972)$ & $(0.087)$ & $(0.209)$ & $(0.062)$ & $(0.062)$ \\
\hline Distance from & -0.083 & -0.308 & 0.002 & -0.002 & -0.001 & -0.001 \\
\hline residence $(\mathrm{km})$ & $(1.071)$ & $(42.252)$ & $(0.001)$ & $(0.086)$ & $(0.061)$ & $(0.061)$ \\
\hline Distance from road & $-20.854^{* *}$ & -2.569 & 0.029 & 0.074 & 0.005 & 0.002 \\
\hline$(\mathrm{km})$ & $(9.577)$ & $(21.924)$ & $(0.024)$ & $(0.054)$ & $(0.016)$ & $(0.014)$ \\
\hline $2012(=1)$ & $121.727^{* * * *}$ & $178.450^{* * * *}$ & $-0.170^{* * * *}$ & $-0.222^{* * *}$ & $-0.124^{* * *}$ & -0.071 \\
\hline & $(27.185)$ & $(51.524)$ & $(0.060)$ & $(0.102)$ & $(0.046)$ & $(0.047)$ \\
\hline Non-random village & $-67.208^{*}$ & $-261.588^{* * *}$ & 0.067 & $0.236^{\text {*** }}$ & $-0.185^{* * *}$ & $-0.198^{* * * *}$ \\
\hline$(=1)$ & $(36.386)$ & $(67.676)$ & $(0.080)$ & $(0.114)$ & $(0.065)$ & $(0.058)$ \\
\hline Material input (000 & & & & & $8 . \mathrm{E}-5^{* * *}$ & \\
\hline IDR/ha) & & & & & (3.E-5) & \\
\hline $\begin{array}{l}\text { Log of labor input } \\
\text { (hours/ha) }\end{array}$ & & & & & & $\begin{array}{l}0.334^{* * *} \\
(0.034)\end{array}$ \\
\hline Constant & & & $3.784^{* * *}$ & $3.487^{* * *}$ & $7.096^{* * *}$ & $4.873^{* * * *}$ \\
\hline & & & $(0.291)$ & $(0.539)$ & $(0.199)$ & $(0.287)$ \\
\hline Chi2 & $139.889^{* * * *}$ & $82.550^{* * * *}$ & $4202.748^{* * * *}$ & $482.462^{\text {***: }}$ & $357.550^{\text {****** }}$ & $1033.791^{\text {*** }}$ \\
\hline Number of obs. & 1101 & 286 & 1015 & 269 & 850 & 846 \\
\hline $\begin{array}{l}\text { Notes: All models w } \\
\text { estimates are shown } \\
\text { dependent variable, a } \\
\text { latent variable change } \\
0.01\end{array}$ & mated $\mathrm{w}$ & lom ef & el estin & ing $\mathrm{da}$ & $\begin{array}{l}2012 \text { anc } \\
\text { Due to le } \\
\text { themselv } \\
p \leq 0.10 \text {, }\end{array}$ & $\begin{array}{l}\text { 15. Coeffi } \\
\text { ensoring o } \\
\text { easure hoy } \\
\leq 0.05 \text {, }\end{array}$ \\
\hline
\end{tabular}


Table A6: Historical forest coverage, land titles, and farm size

\begin{tabular}{|c|c|c|c|c|c|c|c|c|}
\hline & \multicolumn{4}{|c|}{ Plot-level models (systematic land title $=1$ ) } & \multicolumn{4}{|c|}{ Household-level models (log of farm size in ha) } \\
\hline & (1) & (2) & (3) & (4) & (5) & (6) & $(7)$ & $(8)$ \\
\hline & $2 \mathrm{~km}$ radius & $5 \mathrm{~km}$ radius & $10 \mathrm{~km}$ radius & All plots & $2 \mathrm{~km}$ radius & $5 \mathrm{~km}$ radius & $10 \mathrm{~km}$ radius & All plots \\
\hline Share of forested area in 1990 & $\begin{array}{c}-0.180^{* * * *} \\
(0.057)\end{array}$ & $\begin{array}{l}-0.128^{* * *} \\
(0.050)\end{array}$ & $\begin{array}{c}-0.180^{* * * *} \\
(0.065)\end{array}$ & & $\begin{array}{l}0.268^{*} \\
(0.146)\end{array}$ & $\begin{array}{l}0.337^{* *} \\
(0.155)\end{array}$ & $\begin{array}{l}0.453^{* *} \\
(0.198)\end{array}$ & \\
\hline Deforestation $(=1)$ & & & & $\begin{array}{c}-0.060^{* * *} \\
(0.028)\end{array}$ & & & & $\begin{array}{c}0.258^{* * * *} \\
(0.086)\end{array}$ \\
\hline Age of household head (years) & $\begin{array}{c}-0.002 \\
(0.002)\end{array}$ & $\begin{array}{l}-0.001 \\
(0.002)\end{array}$ & $\begin{array}{l}-0.001 \\
(0.001)\end{array}$ & $\begin{array}{l}-0.001 \\
(0.001)\end{array}$ & $\begin{array}{l}0.011^{* *} \\
(0.005)\end{array}$ & $\begin{array}{l}0.011^{\text {** }} \\
(0.005)\end{array}$ & $\begin{array}{l}0.011^{* *} \\
(0.005)\end{array}$ & $\begin{array}{c}0.009^{*} \\
(0.005)\end{array}$ \\
\hline Education (years of schooling) & $\begin{array}{c}0.004 \\
(0.005)\end{array}$ & $\begin{array}{c}1 . \mathrm{E}-4 \\
(0.004)\end{array}$ & $\begin{array}{l}-0.001 \\
(0.004)\end{array}$ & $\begin{array}{l}-0.003 \\
(0.004)\end{array}$ & $\begin{array}{c}0.038^{\text {******* }} \\
(0.012)\end{array}$ & $\begin{array}{c}0.037^{\text {****** }} \\
(0.012)\end{array}$ & $\begin{array}{c}0.037^{* * * * *} \\
(0.012)\end{array}$ & $\begin{array}{c}0.039^{* * * * *} \\
(0.012)\end{array}$ \\
\hline Migrant $(=1)$ & $\begin{array}{l}-0.042 \\
(0.035)\end{array}$ & $\begin{array}{l}-0.020 \\
(0.030)\end{array}$ & $\begin{array}{c}0.009 \\
(0.025)\end{array}$ & $\begin{array}{l}-0.014 \\
(0.029)\end{array}$ & $\begin{array}{c}0.137 \\
(0.101)\end{array}$ & $\begin{array}{c}0.135 \\
(0.101)\end{array}$ & $\begin{array}{c}0.132 \\
(0.101)\end{array}$ & $\begin{array}{c}0.148 \\
(0.101)\end{array}$ \\
\hline Wealth index (initial, quintiles) & $\begin{array}{c}0.003 \\
(0.015)\end{array}$ & $\begin{array}{c}0.009 \\
(0.012)\end{array}$ & $\begin{array}{l}0.018^{*} \\
(0.010)\end{array}$ & $\begin{array}{l}0.029^{* *} \\
(0.011)\end{array}$ & $\begin{array}{c}0.142^{* * * *} \\
(0.034)\end{array}$ & $\begin{array}{l}0.144^{* * * *} \\
(0.034)\end{array}$ & $\begin{array}{c}0.146^{* * * *} \\
(0.034)\end{array}$ & $\begin{array}{c}0.140^{* * * *} \\
(0.034)\end{array}$ \\
\hline Non-random village $(=1)$ & $\begin{array}{l}-0.030 \\
(0.054)\end{array}$ & $\begin{array}{l}-0.041 \\
(0.048)\end{array}$ & $\begin{array}{c}0.005 \\
(0.044)\end{array}$ & $\begin{array}{l}-0.008 \\
(0.051)\end{array}$ & $\begin{array}{c}0.169 \\
(0.137)\end{array}$ & $\begin{array}{c}0.144 \\
(0.136)\end{array}$ & $\begin{array}{c}0.105 \\
(0.137)\end{array}$ & $\begin{array}{c}0.177 \\
(0.136)\end{array}$ \\
\hline Share of migrants in village & $\begin{array}{c}0.212^{* * * * *} \\
(0.074)\end{array}$ & $\begin{array}{c}0.197^{* * * *} \\
(0.069)\end{array}$ & $\begin{array}{l}0.149^{* * *} \\
(0.061)\end{array}$ & $\begin{array}{l}0.184^{* * *} \\
(0.075)\end{array}$ & $\begin{array}{c}0.585^{* * * *} \\
(0.192)\end{array}$ & $\begin{array}{c}0.596^{* * * *} \\
(0.192)\end{array}$ & $\begin{array}{c}0.590^{* * * *} \\
(0.192)\end{array}$ & $\begin{array}{c}0.578^{* * * *} \\
(0.191)\end{array}$ \\
\hline Village wealth index (initial, quintiles) & $\begin{array}{l}-0.017 \\
(0.013)\end{array}$ & $\begin{array}{c}-0.004 \\
(0.013)\end{array}$ & $\begin{array}{l}-3 . \mathrm{E}-5 \\
(0.013)\end{array}$ & $\begin{array}{c}0.001 \\
(0.012)\end{array}$ & $\begin{array}{c}-0.105^{* * * *} \\
(0.033)\end{array}$ & $\begin{array}{c}-0.107^{* * * *} \\
(0.033)\end{array}$ & $\begin{array}{c}-0.111^{* * * *} \\
(0.033)\end{array}$ & $\begin{array}{c}-0.104^{* * * *} \\
(0.033)\end{array}$ \\
\hline Rubber plot (=1) & $\begin{array}{l}-0.059 \\
(0.038)\end{array}$ & $\begin{array}{c}-0.101^{* * * *} \\
(0.025)\end{array}$ & $\begin{array}{c}-0.081^{* * * *} \\
(0.025)\end{array}$ & $\begin{array}{c}-0.097^{* * * *} \\
(0.033)\end{array}$ & & & & \\
\hline Duration of plot ownership (years) & $\begin{array}{c}0.005^{* * * *} \\
(0.002)\end{array}$ & $\begin{array}{c}0.005^{* * *} \\
(0.002)\end{array}$ & $\begin{array}{c}0.004^{* * * *} \\
(0.001)\end{array}$ & $\begin{array}{c}0.006^{* * * *} \\
(0.001)\end{array}$ & & & & \\
\hline Distance from road $(\mathrm{km})$ & $\begin{array}{c}-0.095^{* * *} \\
(0.041)\end{array}$ & $\begin{array}{c}-0.042^{* *} \\
(0.017)\end{array}$ & $\begin{array}{c}-0.025^{* * *} \\
(0.011)\end{array}$ & $\begin{array}{c}-0.023^{* *} \\
(0.011)\end{array}$ & & & & \\
\hline Age of household (years) & & & & & $\begin{array}{c}0.009^{*} \\
(0.005)\end{array}$ & $\begin{array}{l}0.009^{*} \\
(0.005)\end{array}$ & $\begin{array}{l}0.009^{*} \\
(0.005)\end{array}$ & $\begin{array}{l}0.008^{*} \\
(0.005)\end{array}$ \\
\hline Constant & & & & & $\begin{array}{l}-0.544^{*} \\
(0.301)\end{array}$ & $\begin{array}{l}-0.554^{*} \\
(0.300)\end{array}$ & $\begin{array}{l}-0.585^{*} \\
(0.301)\end{array}$ & $\begin{array}{l}-0.514^{*} \\
(0.298)\end{array}$ \\
\hline Regency dummies included & Yes & Yes & Yes & Yes & Yes & Yes & Yes & Yes \\
\hline Chi2 / squared correlation & $74.830^{* * * * *}$ & $95.021^{* * * *}$ & $77.205^{* * * *}$ & $75.126^{* * * *}$ & 0.208 & 0.210 & 0.211 & 0.217 \\
\hline Number of observations & 433 & 660 & 750 & 594 & 462 & 462 & 462 & 462 \\
\hline
\end{tabular}


Table A7: Agricultural productivity and farm size

\begin{tabular}{lcc}
\hline & $(1)$ & $(2)$ \\
\hline Rubber yield (log/ha) & $-0.192^{* * *}$ & $-0.189^{* * *}$ \\
& $(0.059)$ & $(0.060)$ \\
Age of household head (years) & & $0.014^{* * *}$ \\
& & $(0.004)$ \\
Education (years of schooling) & & $0.043^{* * *}$ \\
& & $(0.015)$ \\
Spontaneous migrant (=1) & & 0.039 \\
& & $(0.115)$ \\
Wealth index (initial, quintiles) & & $0.086^{* *}$ \\
& & $(0.038)$ \\
Share of migrants in village & & 0.193 \\
& & $(0.246)$ \\
Village wealth index (initial, & & -0.056 \\
quintiles) & & $(0.036)$ \\
Non-random village (=1) & & \\
Constant & & 0.143 \\
Regency dummies included & 0.088 & $(0.117)$ \\
\hline Observations & $(0.129)$ & $1.644^{* * *}$ \\
\hline Notes: The deps & $(0.528)$ \\
& $2.894^{* * *}$ & Yes \\
\hline & $(0.423)$ & 0.155 \\
& & 349 \\
\hline
\end{tabular}

Notes: The dependent variable in all models is the log of farm size in 2015 measured in hectares. Only farms with productive rubber are included. Coefficient estimates from ordinary least squares models are shown with robust standard errors clustered at village level. ${ }^{*} p \leq 0.10,{ }^{* *} p \leq 0.05,{ }^{* * *} p \leq 0.01$. 


\section{The economics behind an ecological crisis: Livelihood effects of oil palm expansion in Sumatra, Indonesia ${ }^{8}$}

\subsection{Introduction}

The negative environmental effects associated with oil palm (Elaeis guineensis) monocultures in Southeast Asia have received much attention over the last two decades. Studies have shown that oil palm expansion contributed to deforestation and conversion of peat swamps (Koh et al. 2011; Carlson et al. 2012; Wilcove et al. 2013; Stibig et al. 2014; Abood et al. 2015), causing biodiversity loss and disruptions in other ecosystem services (Fitzherbert et al. 2008; Nantha \& Tisdell 2009; Gibbs et al. 2010; Azhar et al. 2011; Lucey \& Hill 2012; Barnes et al. 2014; Guillaume et al. 2015; Konopik et al. 2015; Ganser et al. 2017). In spite of mounting evidence about the negative environmental effects, oil palm expansion has continued in the tropics during the last two decades at a rate unparalleled for any crop in the more recent history of agriculture (Wicke et al. 2011; FAOSTAT 2014; Cramb \& McCarthy 2016a; Vijay et al. 2016). The main reason for this unprecedented expansion is the increase in global demand for vegetable oil due to population and income growth. Increasing demand results in high economic profitability in the palm oil sector (Wilcove \& Koh 2010; Clough et al. 2016; Drescher et al. 2016).

Much of oil palm cultivation is carried out by large companies (Euler et al. 2016a). However, smallholder farmers are also increasingly involved (Rist et al. 2010). Recent studies have estimated that smallholder farmers account for about $40 \%$ of total global palm oil supply with a further rising trend (Bourke \& Harwood 2009; Byerlee et al. 2017; Euler et al. 2017). Unlike large company plantations, smallholder-driven land-use changes are more difficult to monitor and regulate. Hence, policies aimed at more sustainable land-use systems have to build on a thorough understanding of the economic incentives for smallholder farmers.

\footnotetext{
${ }^{8}$ This essay was published as: Kubitza, C.; Krishna, V. V.; Alamsyah, Z. \& Qaim, M. (2018). The Economics Behind an Ecological Crisis: Livelihood Effects of Oil Palm Expansion in Sumatra, Indonesia. Human Ecology, https://doi.org/10.1007/s10745-017-9965-7. CK and VVK developed the research idea, collected the survey data, conducted the regression analyses, and wrote the paper. MQ and ZA commented on data analysis, result interpretation, and revising the paper.
} 
Our study was conducted in Indonesia, the world's leading palm oil producer. Similar to the global pattern, around $40 \%$ of the oil palm area is cultivated by smallholder farmers (Susanti \& Budidarsono 2014; Gatto et al. 2015; Euler et al. 2016b). Several studies have shown that oil palm has a higher labor productivity than alternative cash crops, such as rubber (Bourke \& Harwood 2009; Budidarsono et al. 2012; Clough et al. 2016). However, very few have explicitly analyzed the economic and social effects of oil palm cultivation among smallholder farmers. Extrapolating productivity data from large company plantations is of limited value because the average yields achieved by smallholders are significantly lower than those on large oil palm plantations (Cramb \& McCarthy 2016a; Euler et al. 2016a; Hoffmann et al. 2017; Woittiez et al. 2017). We are aware of only two recent studies that analyzed the livelihood effects of oil palm cultivation in the small-farm sector (Euler et al. 2017; Krishna et al. 2017a). Using cross-sectional data, both studies suggested that cultivating oil palm has a positive effect on household living standards on average. Both also showed that the magnitude of the effect varies depending on the farms' endowment of land, labor, and capital.

While these recent studies are an important step towards better understanding the economics of smallholder oil palm cultivation, we identify two major limitations that are addressed in this article. First, existing studies used cross-sectional data so that the results only provide a snapshot of effects under static conditions. The benefits of cash crop cultivation for farmers critically depend on fluctuating market prices and can hence change over time. Here, we use data from 2012 and 2015 to analyze possible dynamics. In 2012, the international price of palm oil was relatively low compared to the main competing crop - natural rubber (Hevea brasiliensis Muell-Arg.). In subsequent years, the international rubber price declined (Agris 2017), thus adding to the relative profitability of oil palm as an alternative cash crop, such that we expect to observe an increase in the benefits of oil palm cultivation between 2012 and 2015 .

The second limitation of existing studies is that they did not look at possible spillover effects that the cultivation of oil palm by some farmers may have on others in the local context. Spillovers could occur through various mechanisms. For instance, oil palm is less labor-intensive than rubber (Gatto et al. 2015; Euler et al. 2017), which could reduce the local demand for labor and increase the market price of cultivable land and thus may affect employment and other economic opportunities for households in the community. On the 
other hand, the expansion of oil palm is often associated with new public or private sector infrastructure investments that may also be beneficial for local households not directly involved in the palm oil sector. The analysis of spillover effects is possible through the use of spatially explicit regression models, a relatively recent trend in the empirical economics literature (Lewis et al. 2011; Wollni \& Andersson 2014). We are not aware of previous studies that have analyzed economic spillover effects of oil palm cultivation or related land-use changes.

\subsection{Methodology}

\subsubsection{Study area}

This study was conducted in the lowlands of Jambi Province on the island of Sumatra, Indonesia. The region has a tropical humid climate with average temperature of $26.7 \pm 0.2^{\circ} \mathrm{C}$ and annual precipitation of $2235 \pm 381 \mathrm{~mm}$ during the $1991-2011$ period (Drescher et al. 2016). Lowland rainforests largely disappeared and agroforestry systems were significantly downsized in Jambi over the last 30-40 years. The land thus gained was used primarily for rubber and oil palm monocultures (Luskin et al. 2014; Clough et al. 2016; Krishna et al. 2017b).

Oil palm was introduced in Jambi on large state plantations. The diffusion among smallholder farmers started during the late-1980s and early-1990s. During the early stages, smallholder participation was promoted by the Indonesian government through subsidized contract schemes (Rist et al. 2010; Gatto et al. 2017). While subsidized government interventions declined after 1999, smallholder farmers continued to adopt and cultivate oil palm, often independently without any company contracts (Susanti \& Budidarsono 2014). Yet, independent adoption is more often observed in villages where company contracts existed in the past, which is likely due to better access to technical information and to output markets in these settings (Euler et al. 2016b). Official statistics show that around 200,000 households are involved in oil palm cultivation in Jambi, which ranks sixth among Indonesian provinces in terms of crude palm oil production with an estimated oil palm area of over 700,000 ha (Badan Pusat Statistik 2012). At the same time, the province is also 
known for its forest and biodiversity resources; the national parks of Jambi support a number of threatened wildlife species (Luskin et al. 2014).

\subsubsection{Data}

The main unit of observation in our study is the farm household. Survey data were collected from 683 farm households ${ }^{9}$ in two rounds, 2012 and 2015, as part of a larger interdisciplinary research project (for details see Drescher et al 2016). The households were selected for interview following a multi-stage random sampling procedure. Five regencies of Jambi that comprise most of the lowland, non-peat smallholder systems were chosen for the study (Sarolangun, Bungo, Tebo, Batanghari, Muaro Jambi). The survey was carried out in 45 rural villages from these five regencies (40 randomly selected villages and 5 purposively selected ones to facilitate interdisciplinary overlaps). A map of Jambi showing the sample villages is provided in Figure 3.

From each of the selected villages, depending on size, between 6 and 24 farm households were randomly sampled using complete lists of all farm households in 2012. The same households were revisited in 2015. Some sample attrition occurred due to outmigration or deceased household heads, among other reasons, but the attrition rate of $6 \%$ is relatively low. Further randomly selected households in the same villages replaced households that were unavailable in 2015. Using a structured questionnaire, details of all cropping and livestock activities of households during the past 12 months were elicited in both survey rounds. Most farm households in the sample grow either rubber or oil palm or both. Socio-demographic characteristics, details of off-farm income activities, asset endowment, and consumption expenditures on food and non-food goods and services were additionally recorded.

\footnotetext{
${ }^{9}$ In total 700 were interviewed, but 17 farmers were purposively selected due to interdisciplinary overlaps. In the statistical analysis we thus only included 683 farmers.
} 
Figure 3: Map of Jambi Province showing sample villages

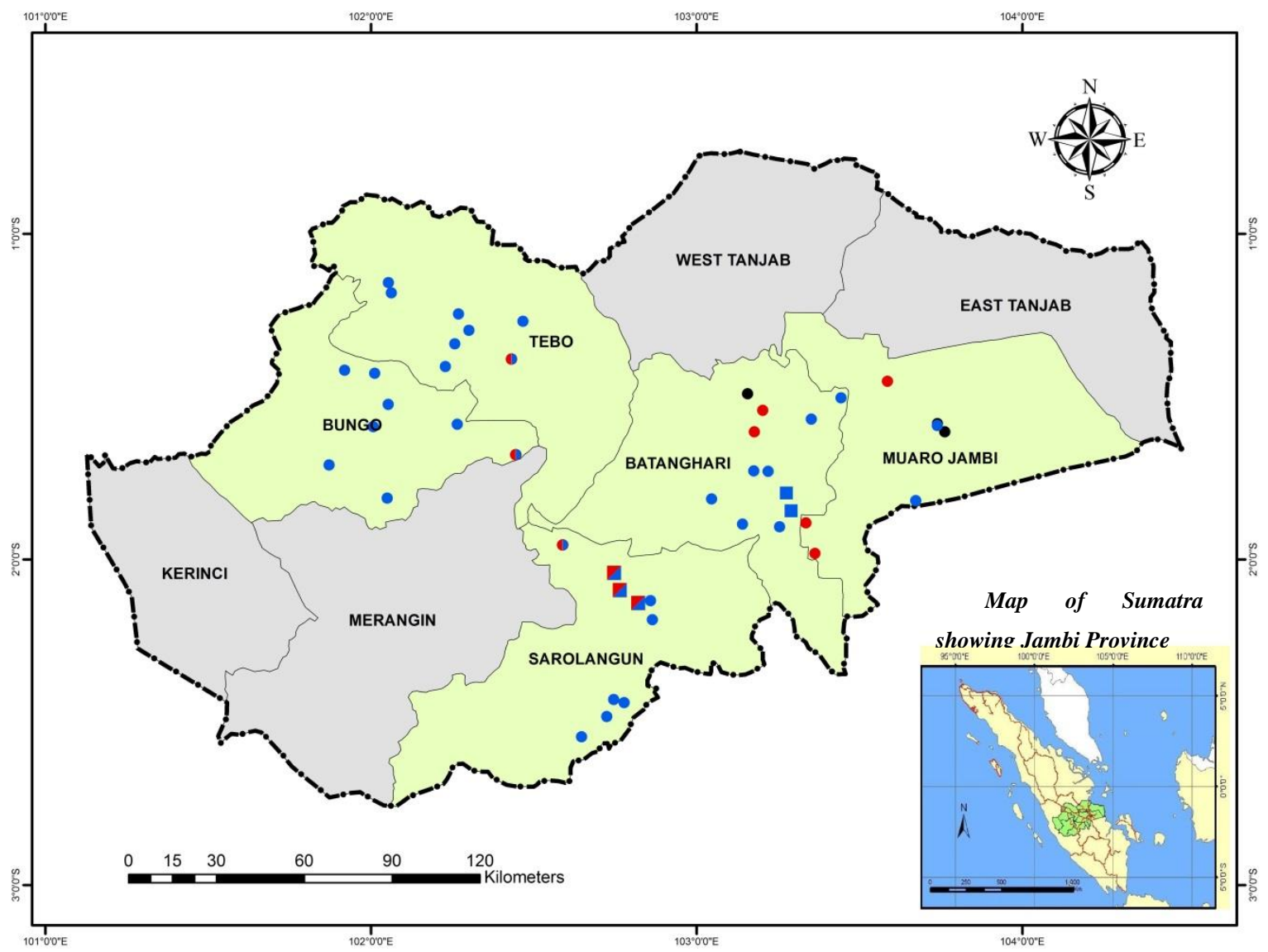

Notes: Dots and squares indicate randomly and purposively selected villages, respectively. Blue dots/squares represent villages where rubber is the main crop (rubber area is larger than oil palm area). Red dots/squares represent villages where oil palm is the main crop.

\subsubsection{Measuring livelihoods}

The value of household consumption (i.e., consumption expenditure) is used as an indicator of household livelihoods (living standards). Being less volatile and less influenced by measurement errors, consumption is considered a better indicator of household living standards than income (Blundell \& Preston 1998). Household consumption expenditure was calculated by summing up the value of all food items and nonfood goods and services consumed by all household members. The expenses on food items were elicited through a seven-day recall, non-food expenditures were captured through monthly or annual recall, depending on the particular goods and services. To make consumption values comparable across households of different size, we calculated annual 
household expenditures per adult equivalent. The 2012 data were adjusted for inflation to enable comparison with the 2015 data.

In addition to total consumption expenditures, we also look at food and non-food consumption separately. The cultivation of oil palm (and other non-food cash crops) can affect food consumption in farm households through the subsistence pathway (possibly less food) and through the income pathway (possibly more food) (Sibhatu et al. 2015). A breakdown by type of expenditure can help better understand the various facets of household livelihoods.

\subsubsection{Estimating effects of oil palm cultivation}

We estimate the effects of oil palm cultivation on household livelihoods by using a standard random-effects model. To account for possible non-linearity, consumption expenditures in household $i$ at time $t\left(c_{i t}\right)$ are expressed in natural logs. $c_{i t}$ is regressed on oil palm cultivation $\left(O P_{i t}\right)$ and a set of $k$ household characteristics $\left(x_{i t k}\right)$ :

$$
c_{i t}=\beta_{0}+\beta_{1} O P_{i t}+\beta_{2 k} x_{i t k}+\mu_{i}+\varepsilon_{i t}
$$

where $\mu_{i}$ is the unobserved time-invariant heterogeneity of the model, and $\varepsilon_{i t}$ is the independent identically distributed error term. We estimate separate models for total consumption expenditures, food expenditures, and non-food expenditures. In all models, we are particularly interested in the estimates for $\beta_{1}$. As oil palm cultivation $\left(O P_{i t}\right)$ is defined as a dummy variable, and the dependent variables are expressed in log terms, the percentage effect of cultivation on consumption is calculated as $\left\{\mathrm{e}^{\widehat{\beta_{1}}-0.5 \widehat{V}\left(\widehat{\beta}_{1}\right)}-1\right\}$, where $\widehat{V}\left(\widehat{\beta_{1}}\right)$ is the estimated variance of $\widehat{\beta_{1}}$ (van Garderen \& Shah 2002). The variance of the percentage change is calculated as $\mathrm{e}^{2 \widehat{\beta_{1}}}\left\{\mathrm{e}^{-\widehat{V}\left(\widehat{\beta_{1}}\right)}-\mathrm{e}^{-2 \widehat{V}\left(\widehat{\beta_{1}}\right)}\right\}$.

One potential problem with the random-effects models in equation (6) is that oil palm cultivators might differ systematically from non-cultivators in terms of certain unobserved characteristics, for example managerial skills or risk attitudes. Such unobserved characteristics may also influence household consumption, which could lead to biased estimates of $\beta_{1}$. Such potential bias due to unobserved heterogeneity is tested by additionally estimating fixed-effects models. Fixed-effects models use differencing 
techniques, thus canceling out any time-invariant unobserved heterogeneity. A Hausman specification test (Hausman 1978) is used to compare the fixed-effects and random-effects estimates. A significant Hausman test statistic would suggest that the random-effects estimates are biased, whereas an insignificant test would mean that the null hypothesis of no bias through time-invariant unobserved heterogeneity cannot be rejected.

\subsubsection{Estimating temporal differences in oil palm effects}

To analyze whether the benefits of oil palm cultivation vary over time, we run separate regression models with cross-sectional data for 2012 and 2015. These cross-sectional models are estimated with ordinary least squares (OLS) and the same set of explanatory variables as in the panel random-effects specifications. We are particularly interested in comparing the effects of oil palm cultivation between 2012 and 2015. Given that the noncultivating households primarily rely on rubber farming and the price of rubber declined between 2012 and 2015, we expect larger benefits of oil palm cultivation in 2015 .

\subsubsection{Estimating spatial patterns and spillovers}

In a separate step of the analysis, we investigate spatial patterns in the estimates. We can differentiate between spatial correlation in the error term and spatial spillover effects of the dependent variables and independent variables. There may be spatial correlation of unobserved factors (e.g., soil fertility). Such spatial dependence would lead to patterns in the error terms and may underestimate the standard errors. We cluster standard errors at the village level to address this problem. Spatial spillover effects can have more severe consequences, as they can lead to biased estimates (Wollni \& Andersson 2014). Standard models of impact assessment, such as those in equation (6), are based on the assumption that outcomes for oil palm cultivators and non-cultivators depend solely on own cultivation, not on the cultivation of oil palm by others. This assumption is violated when spatial spillover effects occur. As discussed above, the cultivation of oil palm by some farmers could also affect others, for instance through changes in factors markets or broader infrastructure developments. To address the potential issue of spatial dependence and empirically assess the existence and magnitude of spillover effects of oil palm cultivation, 
we use a spatial Durbin model with random effects as follows (LeSage \& Pace 2009; Elhorst 2010):

$$
c_{i t}=\beta_{0}+\beta_{1} O P_{i t}+\rho w . O P_{i t}+\beta_{2} x_{i t}+\lambda w \cdot c_{i t}+\mu_{i}+\varepsilon_{i t}
$$

where $w$ is an $n \times n$ spatial weights matrix, based on the inverse distance between the households' residence, and $n$ is the total number of households in the sample. The coefficient $\rho$ measures the lagged effect of cultivation, while $\lambda$, the spatial autoregressive coefficient, measures the lagged effect of consumption expenditures. Since the individual weight of a household decreases with an increasing number of neighbors, the weights matrix is row-standardized, such that for each $i, \sum_{j} w_{i j}=1$.

Taking the partial derivative of the model in equation (7) with respect to oil palm cultivation, the estimate not only shows the livelihood effects oil palm cultivation on cultivating farm households but also the effect on neighboring households. ${ }^{10}$ The first effect is called direct effect and the latter is called indirect effect or spillover effect. We set the threshold of neighborhood distance for spatial effects estimation at $10 \mathrm{~km}$, since spillovers beyond that distance are rather unlikely. The use of a spatial Durbin model and taking the partial derivative is often the preferred method to calculate spillovers (Elhorst 2010). However, critics also underline certain shortcomings with this method, such as the $a$ priori specification of the spatial weights matrix, difficulties in justifying global spillovers, and possible bias due to omitted spatially dependent variables (Corrado \& Fingleton 2012; Halleck Vega \& Elhorst 2015).

${ }^{10}$ Different methods exist to estimate models that include spatial effects. Our estimation is based on maximum likelihood. The regression command used does only allow a balanced panel, we restrict hence our analysis to farmers which were surveyed both in 2012 and 2015. 


\subsection{Results and discussion}

\subsubsection{Descriptive statistics}

Summary statistics are shown in Table 4. The main crop grown by the majority of farmers in the study area is rubber. Oil palm was cultivated by $36 \%$ of the households in 2015. Around $60 \%$ of the oil palm cultivators also produce rubber whereas sample farmers rarely cultivated food crops (Table 4). One of the notable differences between oil palm cultivators and non-cultivators is that the former have significantly larger farms on average. Farmers with large areas of rubber were found to start oil palm cultivation significantly earlier than farmers operating on a smaller scale (Euler et al. 2016b). Farm size in this context refers to all land that farmers reported as owning, including land under formal and informal tenure. Farmers acquired their land mostly through inheritance or inter-vivo transfers, land market purchases, and forest land appropriation. While about half of the plots owned by sample farmers were acquired through market purchases, $18 \%$ were acquired through forest-land appropriation. Other research in Jambi has shown that the role of land acquisition through forest land appropriation has declined over the last 15 years while the role of market purchases has increased (Krishna et al. 2017b).

Table 4 shows that oil palm-cultivating households are more likely to own small nonfarm businesses (e.g., trading, small shops). Because oil palm requires less labor than rubber, oil palm farmers can save family labor. These labor savings allow oil palm cultivators to increase their farm size (if additional land can be acquired) and/or to spend more time in non-farm economic activities. The income generated from these alternative uses of the saved time can be seen as secondary effects of oil palm cultivation. There is no difference in the availability of family labor (number of adults in the household) between oil palm cultivators and non-cultivators. Human capital endowments, which we capture through age and education of the household head, are also similar between the two groups. Oil palm farmers are residing closer to markets, take more credits from formal sources, and are more likely to hold formal titles for the land cultivated. 
Table 4: Differences between oil palm cultivators and non-cultivators

\begin{tabular}{|c|c|c|c|c|}
\hline & \multicolumn{2}{|c|}{ Survey round 2012} & \multicolumn{2}{|c|}{ Survey round 2015} \\
\hline & $\begin{array}{c}\text { Cultivators } \\
{[\mathrm{n}=238]}\end{array}$ & $\begin{array}{c}\text { Non- } \\
\text { cultivators } \\
{[\mathrm{n}=441]}\end{array}$ & $\begin{array}{l}\text { Cultivators } \\
{[\mathrm{n}=248]}\end{array}$ & $\begin{array}{l}\text { Non-cultivators } \\
\quad[\mathrm{n}=435]\end{array}$ \\
\hline \multicolumn{5}{|l|}{$\begin{array}{l}\text { Livelihood indicators: } \\
\text { consumption expenditure } \\
\text { (million IDR/vear/AE) }\end{array}$} \\
\hline $\begin{array}{l}\text { Total consumption } \\
\text { expenditure }\end{array}$ & $\begin{array}{l}24.527^{* *} \\
(15.210)\end{array}$ & $\begin{array}{c}20.090 \\
(31.311)\end{array}$ & $\begin{array}{l}23.956^{* * *} \\
(16.757)\end{array}$ & $\begin{array}{c}17.841 \\
(13.394)\end{array}$ \\
\hline Non-food expenditure & $\begin{array}{l}11.675 \\
(12.710)\end{array}$ & $\begin{array}{c}9.160 \\
(27.643)\end{array}$ & $\begin{array}{l}12.348^{* * * *} \\
(13.982)\end{array}$ & $\begin{array}{c}8.439 \\
(10.738)\end{array}$ \\
\hline Food expenditure & $\begin{array}{c}12.853^{* * *} \\
(6.574)\end{array}$ & $\begin{array}{l}10.931 \\
(6.086)\end{array}$ & $\begin{array}{c}11.608^{* * * *} \\
(6.229)\end{array}$ & $\begin{array}{c}9.402 \\
(4.761)\end{array}$ \\
\hline \multicolumn{5}{|l|}{ Household characteristics } \\
\hline Cultivates rubber $(=1)$ & $0.613^{* * *}$ & 0.946 & $0.601^{* * *}$ & 0.947 \\
\hline Farm size owned (ha) & $\begin{array}{l}5.414^{* * *} \\
(5.289)\end{array}$ & $\begin{array}{c}3.337 \\
(3.955)\end{array}$ & $\begin{array}{l}5.474^{* * *} \\
(5.224)\end{array}$ & $\begin{array}{l}3.208 \\
(4.213)\end{array}$ \\
\hline $\begin{array}{l}\text { Number of adults in the } \\
\text { household }\end{array}$ & $\begin{array}{c}2.849 \\
(1.064)\end{array}$ & $\begin{array}{c}2.980 \\
(1.227)\end{array}$ & $\begin{array}{c}2.964 \\
(1.125)\end{array}$ & $\begin{array}{c}2.922 \\
(1.141)\end{array}$ \\
\hline $\begin{array}{l}\text { Female-headed household } \\
(=1)\end{array}$ & $0.021^{* * * *}$ & 0.068 & $0.028^{* * * *}$ & 0.103 \\
\hline $\begin{array}{l}\text { Age of the household head } \\
\text { (years) }\end{array}$ & $\begin{array}{c}45.508 \\
(12.183)\end{array}$ & $\begin{array}{l}45.773 \\
(12.277)\end{array}$ & $\begin{array}{c}47.661 \\
(10.978)\end{array}$ & $\begin{array}{c}47.485 \\
(11.793)\end{array}$ \\
\hline $\begin{array}{l}\text { Education } \\
\text { schooling) }\end{array}$ & $\begin{array}{c}7.752 \\
(3.604)\end{array}$ & $\begin{array}{c}7.302 \\
(3.680)\end{array}$ & $\begin{array}{l}7.335 \\
(3.526)\end{array}$ & $\begin{array}{l}7.115 \\
(3.780)\end{array}$ \\
\hline Own business (=1) & 0.231 & 0.186 & $0.335^{* * * *}$ & 0.221 \\
\hline Employed (=1) & 0.412 & 0.476 & 0.556 & 0.570 \\
\hline Migrant (=1) & $0.576^{* * *}$ & 0.374 & $0.548^{* * *}$ & 0.379 \\
\hline Transmigrant village $(=1)$ & $0.437^{* * *}$ & 0.236 & $0.411^{* * *}$ & 0.248 \\
\hline $\begin{array}{l}\text { Distance to the nearest } \\
\text { market }(\mathrm{km})\end{array}$ & $\begin{array}{l}5.720^{* * *} \\
(7.482)\end{array}$ & $\begin{array}{c}7.154 \\
(7.359)\end{array}$ & $\begin{array}{l}4.594^{* * *} \\
(5.216)\end{array}$ & $\begin{array}{c}6.036 \\
(6.027)\end{array}$ \\
\hline Formal credit $(=1)$ & $0.353^{* * * *}$ & 0.184 & $0.480^{* * * * *}$ & 0.274 \\
\hline Share of titled land (0-1) & $\begin{array}{l}0.303^{* * *} \\
(0.408)\end{array}$ & $\begin{array}{c}0.172 \\
(0.350)\end{array}$ & $\begin{array}{l}0.339^{* * * *} \\
(0.419)\end{array}$ & $\begin{array}{c}0.224 \\
(0.390)\end{array}$ \\
\hline
\end{tabular}

Notes: Mean values are shown with standard deviations in parentheses. ${ }^{* * *},{ }^{* *}:$ Difference with noncultivators in the same survey round are statistically significant at 0.01 and 0.05 levels, respectively. 
Table 5: Returns to labor and land for oil palm and rubber

\begin{tabular}{|c|c|c|c|c|c|c|c|c|}
\hline & \multicolumn{4}{|c|}{ Survey round 2012} & \multicolumn{4}{|c|}{ Survey round 2015} \\
\hline & \multicolumn{2}{|c|}{ Oil palm } & \multicolumn{2}{|c|}{ Rubber } & \multicolumn{2}{|c|}{ Oil palm } & \multicolumn{2}{|c|}{ Rubber } \\
\hline & $\mathrm{n}$ & $\begin{array}{c}\text { Mean } \\
\text { (Std. dev.) }\end{array}$ & $\mathrm{n}$ & $\begin{array}{c}\text { Mean } \\
(\text { Std. dev.) }\end{array}$ & $\mathrm{n}$ & $\begin{array}{c}\text { Mean } \\
(\text { Std. dev.) }\end{array}$ & $\mathrm{n}$ & $\begin{array}{c}\text { Mean } \\
(\text { Std. dev.) }\end{array}$ \\
\hline $\begin{array}{l}\text { Returns to labor } \\
\text { [000 IDR/hour] }\end{array}$ & 132 & $\begin{array}{l}78.477^{* * *} \\
(73.429)\end{array}$ & 313 & $\begin{array}{c}23.756 \\
(19.105)\end{array}$ & 168 & $\begin{array}{l}46.588^{* * *} \\
(114.399)\end{array}$ & 330 & $\begin{array}{c}10.698 \\
(12.907)\end{array}$ \\
\hline $\begin{array}{l}\text { Returns to land } \\
\text { [Mil.IDR/ha/year] }\end{array}$ & 134 & $\begin{array}{l}13.459^{* * *} \\
(10.085)\end{array}$ & 314 & $\begin{array}{c}19.206 \\
(12.376)\end{array}$ & 168 & $\begin{array}{l}8.221^{* * *} \\
(8.271)\end{array}$ & 330 & $\begin{array}{l}10.260 \\
(7.471)\end{array}$ \\
\hline
\end{tabular}

Notes: Statistical significance was estimated using Kruskal-Wallis equality of populations rank test comparing the variables across the two crops. Only plots surveyed in both rounds were included in the analysis. All unproductive plots were excluded and tree age restricted from 5 to 25 years. Monetary values from 2012 were inflation-adjusted. ${ }^{* * *}$ : Difference between mean values for oil palm and rubber within the same survey round is statistically significant at 0.01 level.

Table 5 shows a comparison of returns to labor and land for oil palm and rubber plots. On average, oil palm cultivation is less profitable than rubber per unit of land but more profitable per unit of labor. These differences between the two crops were less distinct in 2015 than in 2012. The profitability of rubber cultivation declined drastically between the two survey rounds, due to the low market prices for natural rubber prevailing in 2015 (Figure 4). The profitability of oil palm also declined during the same period, albeit less steeply. In 2015, rubber continued to be more profitable per unit of land. Hence rubber is more attractive for households facing land constraints and relatively low opportunity costs of labor time.

The cultivation of oil palm and rubber also differs in terms of inputs other than labor. The literature suggests that the use of chemical inputs, such as fertilizer, is lower in rubber than in oil palm (Budidarsono et al. 2012; Clough et al. 2016). Our data show that that input expenditures vary significantly, not only between crops but also over time (Table A8). The temporal variability is possibly a response to output price changes, which are depicted in Figure 4. Rubber prices decreased from about 30.000 Indonesian Rupiah (IDR) in 2012 to about 15.000 IDR in 2015, resulting in severe reductions in income from rubber cultivation [1 US\$ = 9370 IDR in 2012 and 13390 IDR in 2015 (World Bank 2016)]. Palm oil prices were also lower in 2015 than in 2012, but the difference is less pronounced than in rubber. The higher involvement of all farmers in own non-agricultural business activities and off-farm employment in 2015 (Table 4) can be interpreted as an economic strategy to cope with declining prices in the markets for agricultural cash crops. 
Figure 4: Price movement of rubber and palm between 2012 and 2016

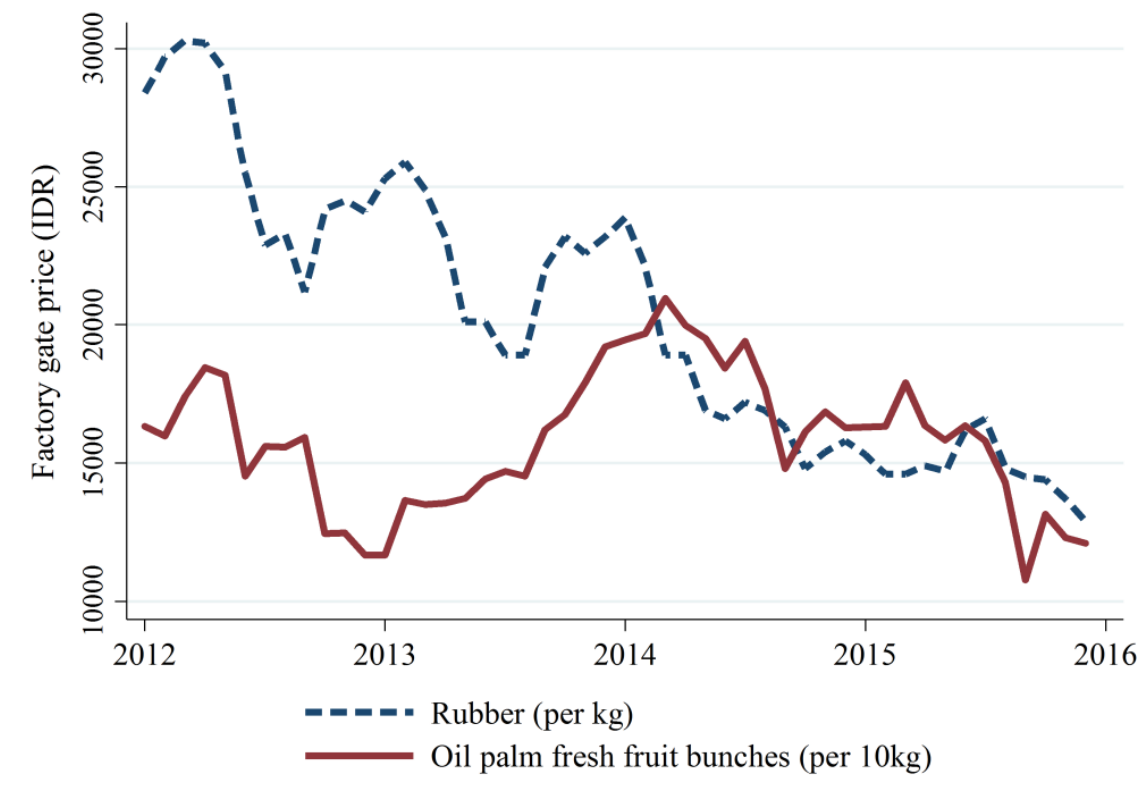

Notes: Rubber price data from Gabungan Perusahaan Karet Indonesia (GAPKINDO), Jambi. Oil palm fresh fruit bunch price data from Dinas Perkebunan, Jambi.

Possibly as a result of the higher involvement in off-farm economic activities, mean household consumption expenditures showed only a moderate decrease between 2012 and 2015, in spite of lower output prices (Table 4). That is, farm households were mostly able to maintain their living standard. The decrease was more pronounced for non-cultivators of oil palm $(-11 \%)$ than for oil palm cultivators $(-2 \%)$, as one would expect given the higher dependence of non-cultivators on rubber prices. Among the non-cultivators of oil palm, the decrease was stronger for non-food expenditures $(-14 \%)$ than for food expenditures $(-8 \%)$. This is not surprising because food is a basic need, so that households try to maintain consumption levels to the extent possible even when their income decreases.

\subsubsection{Average livelihood effects of oil palm cultivation}

The effects of oil palm cultivation on household livelihoods are summarized in Figure 5. The underlying regression models with total consumption expenditures, food expenditures, and non-food expenditures as dependent variables are shown in Tables A9A10. In all three cases, the Hausman test rejects the null hypothesis of no bias through time-invariant unobserved heterogeneity in the random-effects specification. In two of the 
models (total expenditure and non-food expenditure), the effect size of oil palm cultivation is larger in the fixed-effects than in the random-effects specification. However, the data contains only limited variation over time and the random-effects are also more comparable with the cross-section OLS models. Hence, we proceed with interpreting the randomeffects estimates, cautioning that these effects may possibly underestimate the true impacts of oil palm cultivation. We further underline that even by using fixed effects other sources of endogeneity such as reverse causality are not fully addressed.

The random-effects model shows a positive and significant effect of oil palm cultivation on total household consumption. The point estimate in the full model suggests a $14 \%$ improvement in household livelihoods through oil palm cultivation (Figure 5a). The full model controls for farm size and the existence of non-farm businesses in the household, hence the $14 \%$ is the primary effect of cultivation on livelihoods without including possible secondary effects that result from the reallocation of saved labor time. In an alternative model, we exclude the existence of own non-farm businesses from the set of explanatory variables. The point estimate of oil palm cultivation does not change much, suggesting that secondary effects from reallocating household labor to non-farm businesses are small. In yet another model we exclude farm size as explanatory variable. In this model, the point estimate of oil palm cultivation increases to $22 \%$ (Figure 5a), suggesting that there are positive secondary effects on household livelihoods resulting from farm size increases. As mentioned above, the lower labor requirement in oil palm allows households to cultivate additional land, thus further increasing the income from farming.

The breakdown of household expenditure types shows that the effect of oil palm cultivation is positive and significant on both food consumption (Figure 5c) and non-food consumption (Figure 5b). Yet, the effect on non-food consumption (19\%) is larger than the effect on food consumption (10\%). This difference is expected. Most farm households in the sample are above the poverty line (Badan Pusat Statistik 2014) and not chronically food-insecure. Hence, a larger share of the additional income is spent on non-food goods and services. 


\subsubsection{Temporal differences}

The results of the cross-sectional OLS models for 2012 and 2015 are also summarized in Figure 5. The models include farm size and own non-farm businesses as control variables, so that only the primary effects of oil palm cultivation are considered. In both years, oil palm cultivation had positive and significant effects on household livelihoods, but the effects were larger in 2015 than in 2012. In 2012, the average effect on total household consumption was 11\%, whereas in 2015 it was 17\% (Figure 5a). This increase in the percentage effect is not because the income from oil palm increased over time in absolute terms. In fact, Table 5 showed that the absolute profitability of oil palm was lower in 2015 than in 2012 due to declining output prices. However, the relative profitability of oil palm increased because the decline in rubber prices was stronger than the decline in palm oil prices.

The breakdown by food and non-food consumption expenditures in these OLS models shows another interesting result. The effect of oil palm cultivation on both types of expenditures was larger in 2015 than in 2012 (Figures 5b and 5c), but the relative increase in the mean effect was stronger for food consumption (63\%) than for non-food consumption $(31 \%)$. These patterns suggest that oil palm cultivation has helped farm households reduce the risk of food insecurity during the rubber price crisis. 
Figure 5: Livelihood effects of oil palm cultivation

(a) Total household consumption

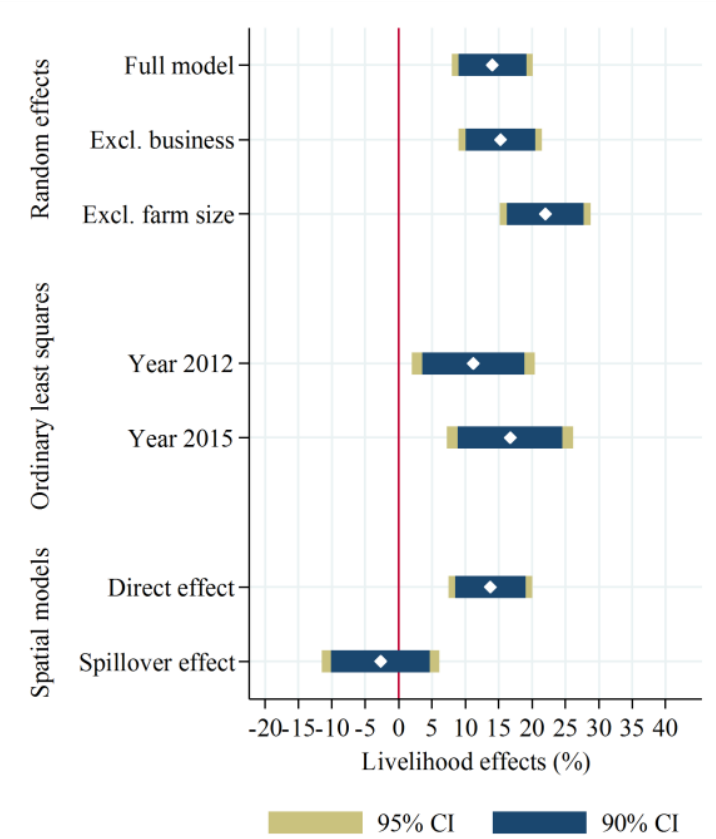

(b) Non-food consumption

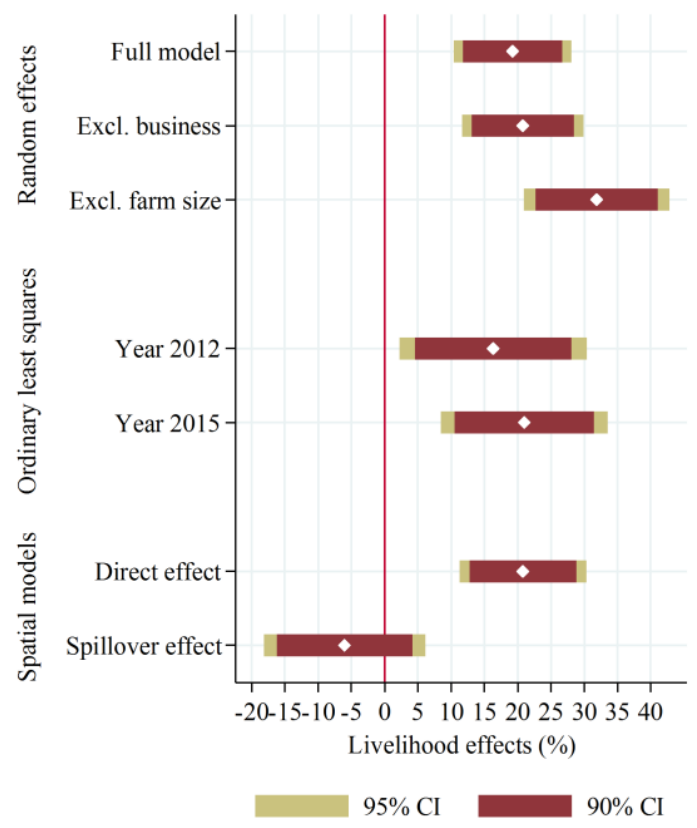

(c) Food consumption

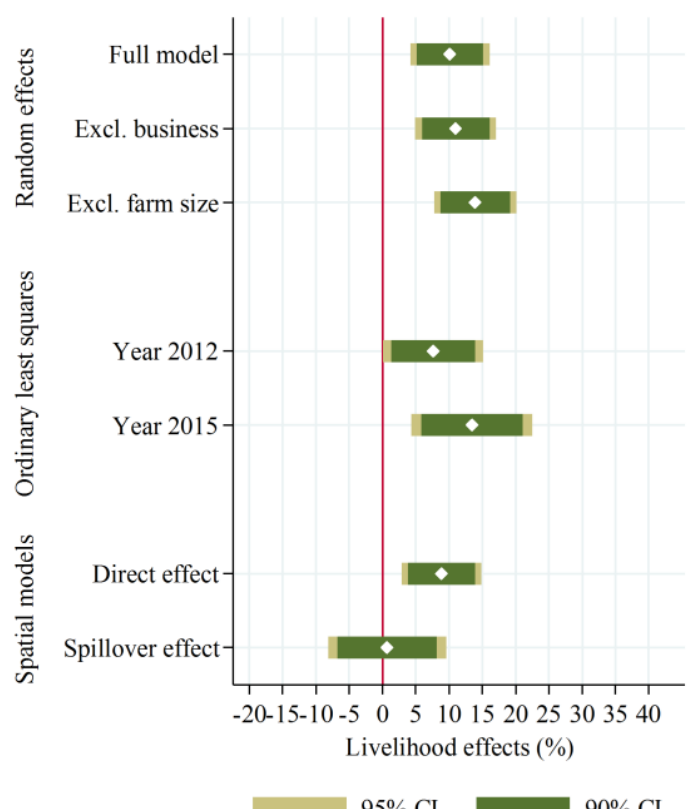

Notes: Consumption is measured in terms of annual household expenditures for food and non-food goods and services per adult equivalent. Average marginal effects are shown. The underlying regression models are presented in Tables A9, A10, and A11. 


\subsubsection{Spillover effects}

Estimates from the spatial models are summarized in the lower parts of Figure 5. The direct effects are those that occur through own cultivation of oil palm, but now controlling for spatial dependence. The estimates are positive, statistically significant, and similar in magnitude to the ones from the random-effects specifications (full models). Hence, controlling for spatial dependence does not alter the findings. The estimated spillover effects are small and statistically insignificant, i.e., during the study period the livelihoods of neighboring households were not significantly influenced through the cultivation of oil palm by others. The insignificant estimates do not necessarily imply that the expansion of oil palm would leave, for example, non-cultivating households completely unaffected. It is possible that negative spillovers through some mechanisms were compensated by positive spillovers through other mechanisms. But our findings suggest that the total spillovers did not affect the livelihoods of other farm households in a significantly positive or negative way.

\subsection{Conclusion}

Our empirical study with farm household data from Jambi Province, Sumatra, shows that the expansion of oil palm has affected the livelihoods of cultivating farm households in a positive way. This result is consistent with previous research in Sumatra (Euler et al. 2017; Krishna et al. 2017a), although previous studies had not used panel data for the analysis of impacts, as we have done here.

Oil palm contributes to higher household consumption, including food and non-food expenditures. On average, oil palm does not generate higher profits per unit of land than rubber, the main alternative crop. However, oil palm requires less labor, so that oil palmcultivating households are able to manage larger areas of land with the same labor input. When holding farm size constant, the average improvement in household livelihoods through oil palm cultivation was $14 \%$. When letting farm size vary, the effect increased to $22 \%$. These results suggest that parts of the total economic benefits are indeed the result of oil palm cultivators expanding their farm size. We also showed that the effects of oil palm cultivation vary over time. Due to the sharp price decline in international rubber markets, 
the relative improvements in household livelihoods through cultivating oil palm increased between 2012 and 2015. We did not find significant spillover effects on the livelihoods of neighboring farm households.

Our results refute the commonly held perception that oil palm diffusion would only benefit large plantation companies and would affect the livelihoods of the local population in a negative way. Our data are not representative for the entire population of Jambi, because we only looked at farm households. But most of the farm households benefit significantly. Unquestionably, oil palm monocultures are also associated with environmental problems. And the fact that the labor savings through oil palm cultivation allow smallholders to expand their farm size may contribute to additional forest clearing when effective rules to curb deforestation are not in place. Policies aimed at more sustainable land use are required. But such policies cannot ignore the economic benefits of oil palm cultivation for local farmers. Only when the incentive structures of local smallholders are properly understood, can socioeconomic and environmental goals be reconciled through appropriate policy interventions.

Data Availability: The data used in this study are archived with openly accessible, keyword-searchable metadata and data holder contact details for data requests (https://efforts-is.uni-goettingen.de). Datasets used in this study have the following identification numbers: 12620, 13500, 13501, 13520, 13660, 13642, 13643, 13644, 13647, 13648, 13649, 13650, 13651 (household-level data); 13521, 13600, 13601, 13620 (plotlevel data); 13680 (village-level data). 


\subsection{Appendix}

Table A8: Differences in input use between rubber and oil palm cultivation in 2012 and 2015

\begin{tabular}{|c|c|c|c|c|}
\hline \multirow{2}{*}{$\begin{array}{l}\text { Expenses } \\
\text { ('000 IDR/year) }\end{array}$} & \multicolumn{2}{|c|}{ Survey round 2012} & \multicolumn{2}{|c|}{ Survey round 2015} \\
\hline & $\begin{array}{l}\text { Rubber } \\
\text { farmer } \\
{[n=560]}\end{array}$ & $\begin{array}{c}\text { Oil palm } \\
\text { farmer } \\
{[n=226]}\end{array}$ & $\begin{array}{l}\text { Rubber } \\
\text { Farmer } \\
{[\mathrm{n}=561]}\end{array}$ & $\begin{array}{l}\text { Oil palm } \\
\text { farmer } \\
{[n=248]}\end{array}$ \\
\hline On chemical & $980.667^{* * * *}$ & 4842.611 & $505.859^{* * * *}$ & 4264.176 \\
\hline fertilizers & (6938.109) & (9739.918) & $(4017.621)$ & $(5909.125)$ \\
\hline On herbicides & $639.284^{\# \# \#}$ & $800.360^{\# \# \#}$ & $273.322^{* * *}$ & 524.459 \\
\hline & $(1762.845)$ & $(1326.163)$ & $(773.078)$ & $(940.860)$ \\
\hline
\end{tabular}

Notes: Mean values are shown with standard deviations in parentheses. ${ }^{* * *}:$ Difference between rubber and oil palm in the same year is statistically significant at 0.01 level. \#\#\#: Difference with corresponding value for the same crop in 2015 is statistically significant at 0.01 level. 
Table A9: Impact of oil palm cultivation on total consumption expenditures

\begin{tabular}{|c|c|c|c|c|c|c|c|c|}
\hline & $\begin{array}{c}(1) \\
\text { Fixed effects }\end{array}$ & $\begin{array}{c}(2) \\
\text { Random effects: } \\
\text { Full model }\end{array}$ & $\begin{array}{c}(3) \\
\text { Random Effects: } \\
\text { Without own } \\
\text { business } \\
\end{array}$ & $\begin{array}{c}(4) \\
\text { Random Effects: } \\
\text { Without farm } \\
\text { size } \\
\end{array}$ & $\begin{array}{c}\text { (5) } \\
\text { Ordinary least } \\
\text { squares } 2012\end{array}$ & $\begin{array}{c}6) \\
\text { Ordinary least } \\
\text { squares } 2015\end{array}$ & $\begin{array}{c}(7) \\
\text { Spatial Durbin } \\
\text { model: } \\
\text { Direct effect }\end{array}$ & $\begin{array}{c}\text { (8) } \\
\text { Spatial Durbin } \\
\text { model: Indirect } \\
\text { effect }\end{array}$ \\
\hline Oil palm cultivation (dummy) & $\begin{array}{l}0.229^{*} \\
(0.132)\end{array}$ & $\begin{array}{l}0.141^{* * *} \\
(0.031)\end{array}$ & $\begin{array}{l}0.153^{* * *} \\
(0.032)\end{array}$ & $\begin{array}{l}0.220^{* * * *} \\
(0.035)\end{array}$ & $\begin{array}{l}0.112^{* *} \\
(0.046)\end{array}$ & $\begin{array}{l}0.167^{* * *} \\
(0.047)\end{array}$ & $\begin{array}{l}0.138^{* * *} \\
(0.032)\end{array}$ & $\begin{array}{l}-0.027 \\
(0.044)\end{array}$ \\
\hline Total farm size (ha) & $\begin{array}{c}0.006 \\
(0.012)\end{array}$ & $\begin{array}{l}0.032^{* * * *} \\
(0.004)\end{array}$ & $\begin{array}{l}0.033^{* * *} \\
(0.004)\end{array}$ & & $\begin{array}{l}0.039^{* * *} \\
(0.007)\end{array}$ & $\begin{array}{l}0.026^{* * *} \\
(0.004)\end{array}$ & $\begin{array}{l}0.031^{* * *} \\
(0.004)\end{array}$ & $\begin{array}{c}0.003 \\
(0.002)\end{array}$ \\
\hline Number of adults in household & $\begin{array}{l}-0.078^{* * * *} \\
(0.020)\end{array}$ & $\begin{array}{l}-0.053^{* * *} \\
(0.011)\end{array}$ & $\begin{array}{c}-0.046^{* * *} \\
(0.011)\end{array}$ & $\begin{array}{c}-0.046^{* * *} \\
(0.011)\end{array}$ & $\begin{array}{l}-0.031^{*} \\
(0.016)\end{array}$ & $\begin{array}{c}-0.067^{* * *} \\
(0.016)\end{array}$ & $\begin{array}{l}-0.053^{* * * *} \\
(0.011)\end{array}$ & $\begin{array}{l}-0.005 \\
(0.003)\end{array}$ \\
\hline Own business $(=1)$ & $\begin{array}{l}0.136^{* * *} \\
(0.053)\end{array}$ & $\begin{array}{l}0.205^{* * *} \\
(0.038)\end{array}$ & & $\begin{array}{l}0.219^{* * * *} \\
(0.039)\end{array}$ & $\begin{array}{l}0.242^{* * *} \\
(0.059)\end{array}$ & $\begin{array}{l}0.180^{* * *} \\
(0.050)\end{array}$ & $\begin{array}{l}0.196^{* * *} \\
(0.038)\end{array}$ & $\begin{array}{c}0.016 \\
(0.011)\end{array}$ \\
\hline Employment (=1) & $\begin{array}{l}0.074^{*} \\
(0.042)\end{array}$ & $\begin{array}{c}0.006 \\
(0.023)\end{array}$ & $\begin{array}{l}-0.018 \\
(0.023)\end{array}$ & $\begin{array}{l}-0.039 \\
(0.026)\end{array}$ & $\begin{array}{c}0.030 \\
(0.037)\end{array}$ & $\begin{array}{l}-0.053 \\
(0.039)\end{array}$ & $\begin{array}{c}0.006 \\
(0.023)\end{array}$ & $\begin{array}{l}1.9 \mathrm{e}-04 \\
(0.002)\end{array}$ \\
\hline $\begin{array}{l}\text { Wave (dummy; } 0=2012 ; 1= \\
\text { 2015) }\end{array}$ & $\begin{array}{l}0.091^{* * *} \\
(0.025)\end{array}$ & $\begin{array}{l}0.095^{* * * *} \\
(0.038)\end{array}$ & $\begin{array}{l}0.081^{* *} \\
(0.037)\end{array}$ & $\begin{array}{l}0.096^{* * *} \\
(0.039)\end{array}$ & & & $\begin{array}{l}0.084^{* * * *} \\
(0.034)\end{array}$ & $\begin{array}{c}0.007 \\
(0.006)\end{array}$ \\
\hline Female headed household $(=1)$ & & $\begin{array}{l}-0.116^{* *} \\
(0.052)\end{array}$ & $\begin{array}{l}-0.120^{* *} \\
(0.049)\end{array}$ & $\begin{array}{l}-0.127^{* * *} \\
(0.052)\end{array}$ & $\begin{array}{l}-0.079 \\
(0.085)\end{array}$ & $\begin{array}{l}-0.113 \\
(0.069)\end{array}$ & $\begin{array}{l}-0.115^{*} \\
(0.060)\end{array}$ & $\begin{array}{l}-0.009 \\
(0.009)\end{array}$ \\
\hline Age of household head (Years) & & $\begin{array}{c}0.009 \\
(0.007)\end{array}$ & $\begin{array}{c}0.008 \\
(0.007)\end{array}$ & $\begin{array}{l}0.018^{* *} \\
(0.008)\end{array}$ & $\begin{array}{l}-0.005 \\
(0.008)\end{array}$ & $\begin{array}{l}0.028^{* *} \\
(0.011)\end{array}$ & $\begin{array}{c}0.008 \\
(0.008)\end{array}$ & $\begin{array}{c}4.6 \mathrm{e}-04 \\
(8.1 \mathrm{e}-04)\end{array}$ \\
\hline Age of household head squared & & $\begin{array}{c}-9.5 \mathrm{e}-05 \\
(7.1 \mathrm{e}-05)\end{array}$ & $\begin{array}{c}-9.2 \mathrm{e}-05 \\
(7.1 \mathrm{e}-05)\end{array}$ & $\begin{array}{l}-1.7 \mathrm{e}-04^{* *} \\
(7.9 \mathrm{e}-05)\end{array}$ & $\begin{array}{c}4.4 \mathrm{e}-05 \\
(8.4 \mathrm{e}-05)\end{array}$ & $\begin{array}{c}-2.9 \mathrm{e}-04^{* * *} \\
(1.1 \mathrm{e}-04)\end{array}$ & $\begin{array}{c}-7.8 \mathrm{e}-05 \\
(7.8 \mathrm{e}-05)\end{array}$ & $\begin{array}{l}-4.8 \mathrm{e}-06 \\
(8.1 \mathrm{e}-06)\end{array}$ \\
\hline Education (years of schooling) & & $\begin{array}{l}0.022^{* * * *} \\
(0.004)\end{array}$ & $\begin{array}{l}0.022^{* * * *} \\
(0.004)\end{array}$ & $\begin{array}{l}0.028^{* * *} \\
(0.005)\end{array}$ & $\begin{array}{l}0.020^{* * *} \\
(0.005)\end{array}$ & $\begin{array}{l}0.026^{* * *} \\
(0.006)\end{array}$ & $\begin{array}{l}0.024^{* * * *} \\
(0.004)\end{array}$ & $\begin{array}{c}0.002 \\
(0.001)\end{array}$ \\
\hline Non-random village $(=1)$ & & $\begin{array}{l}0.070^{* *} \\
(0.035)\end{array}$ & $\begin{array}{l}0.069^{* *} \\
(0.029)\end{array}$ & $\begin{array}{l}0.130^{* * * *} \\
(0.044)\end{array}$ & $\begin{array}{c}0.054 \\
(0.076)\end{array}$ & $\begin{array}{c}0.074 \\
(0.066)\end{array}$ & $\begin{array}{l}0.066^{*} \\
(0.036)\end{array}$ & $\begin{array}{c}0.005 \\
(0.005)\end{array}$ \\
\hline Spontaneous migrant $(=1)$ & & $\begin{array}{l}0.069^{*} \\
(0.040)\end{array}$ & $\begin{array}{l}0.071^{*} \\
(0.041)\end{array}$ & $\begin{array}{c}0.067 \\
(0.046)\end{array}$ & $\begin{array}{c}0.076 \\
(0.056)\end{array}$ & $\begin{array}{c}0.077 \\
(0.052)\end{array}$ & $\begin{array}{l}0.078^{* *} \\
(0.035)\end{array}$ & $\begin{array}{c}0.006 \\
(0.005)\end{array}$ \\
\hline Transmigrant village $(=1)$ & & $\begin{array}{c}0.059 \\
(0.051)\end{array}$ & $\begin{array}{c}0.043 \\
(0.054)\end{array}$ & $\begin{array}{c}0.047 \\
(0.063)\end{array}$ & $\begin{array}{l}0.114^{*} \\
(0.065)\end{array}$ & $\begin{array}{c}0.002 \\
(0.083)\end{array}$ & $\begin{array}{c}0.056 \\
(0.053)\end{array}$ & $\begin{array}{c}0.004 \\
(0.005)\end{array}$ \\
\hline $\begin{array}{l}\text { Distance to the nearest market } \\
(\mathrm{km})\end{array}$ & & $\begin{array}{l}-0.001 \\
(0.001)\end{array}$ & $\begin{array}{l}-0.002 \\
(0.001)\end{array}$ & $\begin{array}{l}-0.002 \\
(0.002)\end{array}$ & $\begin{array}{l}-0.003 \\
(0.002)\end{array}$ & $\begin{array}{c}0.001 \\
(0.003)\end{array}$ & $\begin{array}{l}-0.001 \\
(0.001)\end{array}$ & $\begin{array}{l}-1.0 \mathrm{e}-04 \\
(1.6 \mathrm{e}-04)\end{array}$ \\
\hline Access to formal credit $(=1)$ & & $\begin{array}{l}0.054^{*} \\
(0.032)\end{array}$ & $\begin{array}{l}0.074^{* *} \\
(0.031)\end{array}$ & $\begin{array}{l}0.068^{* *} \\
(0.033)\end{array}$ & $\begin{array}{l}0.040 \\
(0.043)\end{array}$ & $\begin{array}{l}0.075^{*} \\
(0.038)\end{array}$ & $\begin{array}{l}0.058^{*} \\
(0.032)\end{array}$ & $\begin{array}{l}0.005 \\
(0.005)\end{array}$ \\
\hline $\begin{array}{l}\text { Share of land with systematic } \\
\text { land title (ratio) }\end{array}$ & & $\begin{array}{c}0.069 \\
(0.048)\end{array}$ & $\begin{array}{c}0.074 \\
(0.050)\end{array}$ & $\begin{array}{c}0.039 \\
(0.055)\end{array}$ & $\begin{array}{c}0.025 \\
(0.059)\end{array}$ & $\begin{array}{c}0.099 \\
(0.074)\end{array}$ & $\begin{array}{c}0.067 \\
(0.047)\end{array}$ & $\begin{array}{c}0.007 \\
(0.007)\end{array}$ \\
\hline Constant & $\begin{array}{l}28.696 \\
(22.857)\end{array}$ & $\begin{array}{l}9.231^{* * *} \\
(9.231) \\
\end{array}$ & $\begin{array}{l}9.282^{* * *} \\
(9.282) \\
\end{array}$ & $\begin{array}{l}9.042^{* * *} \\
(9.042) \\
\end{array}$ & $\begin{array}{l}9.574^{* * *} \\
(9.574) \\
\end{array}$ & $\begin{array}{l}8.841^{* * *} \\
(8.841) \\
\end{array}$ & & \\
\hline $\mathrm{F}, \chi^{2}, \mathrm{R}^{2}$ & 6.075 & 496.2 & 498.1 & 436.0 & 13.69 & 19.68 & 0.255 & 0.255 \\
\hline Observations & 1278 & 1361 & 1361 & 1361 & 679 & 682 & 1274 & 1274 \\
\hline
\end{tabular}


Table A10: Impact of oil palm cultivation on non-food consumption expenditures

\begin{tabular}{|c|c|c|c|c|c|c|c|c|}
\hline & $\begin{array}{c}(1) \\
\text { Fixed effects }\end{array}$ & $\begin{array}{c}(2) \\
\text { Random effects: } \\
\text { Full model }\end{array}$ & $\begin{array}{c}(3) \\
\text { Random Effects: } \\
\text { Without own } \\
\text { business } \\
\end{array}$ & $\begin{array}{c}\text { (4) } \\
\text { Random Effects: } \\
\text { Without farm } \\
\text { size } \\
\end{array}$ & $\begin{array}{c}\text { (5) } \\
\text { Ordinary least } \\
\text { squares } 2012\end{array}$ & $\begin{array}{c}6) \\
\text { Ordinary least } \\
\text { squares } 2015\end{array}$ & $\begin{array}{c}(7) \\
\text { Spatial Durbin } \\
\text { model: } \\
\text { Direct effect } \\
\end{array}$ & $\begin{array}{c}\text { (8) } \\
\text { Spatial Durbin } \\
\text { model: Indirect } \\
\text { effect }\end{array}$ \\
\hline Oil palm cultivation (dummy) & $\begin{array}{l}0.377^{* *} \\
(0.222)\end{array}$ & $\begin{array}{l}0.192^{* * *} \\
(0.045)\end{array}$ & $\begin{array}{l}0.207^{* * *} \\
(0.047)\end{array}$ & $\begin{array}{l}0.319^{* * *} \\
(0.056)\end{array}$ & $\begin{array}{l}0.163^{* *} \\
(0.070)\end{array}$ & $\begin{array}{l}0.210^{* * *} \\
(0.062)\end{array}$ & $\begin{array}{l}0.208^{* * *} \\
(0.049)\end{array}$ & $\begin{array}{l}-0.060 \\
(0.061)\end{array}$ \\
\hline Total farm size (ha) & $\begin{array}{c}0.020 \\
(0.018)\end{array}$ & $\begin{array}{l}0.048^{* * *} \\
(0.004)\end{array}$ & $\begin{array}{l}0.050^{* * *} \\
(0.004)\end{array}$ & & $\begin{array}{l}0.055^{* * *} \\
(0.008)\end{array}$ & $\begin{array}{l}0.042^{* * *} \\
(0.006)\end{array}$ & $\begin{array}{l}0.046^{* * *} \\
(0.004)\end{array}$ & $\begin{array}{c}0.003 \\
(0.002)\end{array}$ \\
\hline Number of adults in household & $\begin{array}{l}-0.124^{* * *} \\
(0.029)\end{array}$ & $\begin{array}{l}-0.066^{* * *} \\
(0.014)\end{array}$ & $\begin{array}{l}-0.058^{* * * *} \\
(0.014)\end{array}$ & $\begin{array}{l}-0.057^{* * *} \\
(0.015)\end{array}$ & $\begin{array}{l}-0.034 \\
(0.023)\end{array}$ & $\begin{array}{l}-0.083^{* * *} \\
(0.022)\end{array}$ & $\begin{array}{l}-0.070^{* * *} \\
(0.015)\end{array}$ & $\begin{array}{l}-0.005 \\
(0.003)\end{array}$ \\
\hline Own business $(=1)$ & $\begin{array}{c}0.119 \\
(0.076)\end{array}$ & $\begin{array}{l}0.252^{* * *} \\
(0.050)\end{array}$ & & $\begin{array}{l}0.274^{* * *} \\
(0.052)\end{array}$ & $\begin{array}{l}0.269^{* * *} \\
(0.080)\end{array}$ & $\begin{array}{l}0.254^{* * *} \\
(0.063)\end{array}$ & $\begin{array}{l}0.240^{* * *} \\
(0.050)\end{array}$ & $\begin{array}{c}0.015 \\
(0.011)\end{array}$ \\
\hline Employment (=1) & $\begin{array}{l}0.128^{* *} \\
(0.064)\end{array}$ & $\begin{array}{c}0.014 \\
(0.036)\end{array}$ & $\begin{array}{l}-0.015 \\
(0.035)\end{array}$ & $\begin{array}{l}-0.053 \\
(0.037)\end{array}$ & $\begin{array}{c}0.020 \\
(0.057)\end{array}$ & $\begin{array}{l}-0.036 \\
(0.058)\end{array}$ & $\begin{array}{c}0.026 \\
(0.038)\end{array}$ & $\begin{array}{c}0.001 \\
(0.003)\end{array}$ \\
\hline $\begin{array}{l}\text { Wave (dummy; } 0=2012 ; 1= \\
\text { 2015) }\end{array}$ & $\begin{array}{c}0.029 \\
(0.035)\end{array}$ & $\begin{array}{c}0.039 \\
(0.049)\end{array}$ & $\begin{array}{c}0.023 \\
(0.048)\end{array}$ & $\begin{array}{c}0.040 \\
(0.051)\end{array}$ & & & $\begin{array}{c}0.031 \\
(0.048)\end{array}$ & $\begin{array}{c}0.002 \\
(0.005)\end{array}$ \\
\hline Female headed household $(=1)$ & & $\begin{array}{l}-0.121 \\
(0.080)\end{array}$ & $\begin{array}{l}-0.126 \\
(0.079)\end{array}$ & $\begin{array}{l}-0.137 \\
(0.083)\end{array}$ & $\begin{array}{c}0.011 \\
(0.157)\end{array}$ & $\begin{array}{l}-0.174 \\
(0.096)\end{array}$ & $\begin{array}{l}-0.120 \\
(0.097)\end{array}$ & $\begin{array}{l}-0.007 \\
(0.009)\end{array}$ \\
\hline Age of household head (Years) & & $\begin{array}{l}0.039^{* * *} \\
(0.010)\end{array}$ & $\begin{array}{l}0.038^{* * *} \\
(0.010)\end{array}$ & $\begin{array}{l}0.053^{* * *} \\
(0.011)\end{array}$ & $\begin{array}{l}0.026^{* *} \\
(0.011)\end{array}$ & $\begin{array}{l}0.058^{* * *} \\
(0.017)\end{array}$ & $\begin{array}{l}0.038^{* * * *} \\
(0.012)\end{array}$ & $\begin{array}{c}0.003 \\
(0.002)\end{array}$ \\
\hline Age of household head squared & & $\begin{array}{l}-4.4 \mathrm{e}-04^{* * *} \\
(9.9 \mathrm{e}-05)\end{array}$ & $\begin{array}{l}-4.3 \mathrm{e}-04^{* * *} \\
(9.8 \mathrm{e}-05)\end{array}$ & $\begin{array}{l}-5.4 \mathrm{e}-04^{* * *} \\
(1.1 \mathrm{e}-04)\end{array}$ & $\begin{array}{l}-3.2 \mathrm{e}-04^{* * *} \\
(1.1 \mathrm{e}-04)\end{array}$ & $\begin{array}{l}-6.3 \mathrm{e}-04^{* * * *} \\
(1.7 \mathrm{e}-04)\end{array}$ & $\begin{array}{l}-4.3 \mathrm{e}-04^{* * *} \\
(1.2 \mathrm{e}-04)\end{array}$ & $\begin{array}{l}-2.8 \mathrm{e}-05 \\
(1.9 \mathrm{e}-05)\end{array}$ \\
\hline Education (years of schooling) & & $\begin{array}{l}0.033^{* * *} \\
(0.006)\end{array}$ & $\begin{array}{l}0.033^{* * *} \\
(0.006)\end{array}$ & $\begin{array}{l}0.042^{* * *} \\
(0.006)\end{array}$ & $\begin{array}{l}0.033^{* * *} \\
(0.008)\end{array}$ & $\begin{array}{l}0.035^{* * *} \\
(0.009)\end{array}$ & $\begin{array}{l}0.034^{* * *} \\
(0.006)\end{array}$ & $\begin{array}{c}0.002 \\
(0.002)\end{array}$ \\
\hline Non-random village $(=1)$ & & $\begin{array}{l}-0.016 \\
(0.062)\end{array}$ & $\begin{array}{l}-0.016 \\
(0.055)\end{array}$ & $\begin{array}{c}0.068 \\
(0.066)\end{array}$ & $\begin{array}{c}0.004 \\
(0.084)\end{array}$ & $\begin{array}{l}-0.043 \\
(0.089)\end{array}$ & $\begin{array}{c}0.001 \\
(0.061)\end{array}$ & $\begin{array}{l}5.7 \mathrm{e}-05 \\
(0.005)\end{array}$ \\
\hline Spontaneous migrant $(=1)$ & & $\begin{array}{l}0.159^{* * *} \\
(0.064)\end{array}$ & $\begin{array}{l}0.162^{* * *} \\
(0.065)\end{array}$ & $\begin{array}{l}0.156^{* *} \\
(0.074)\end{array}$ & $\begin{array}{l}0.154^{*} \\
(0.088)\end{array}$ & $\begin{array}{l}0.177^{* *} \\
(0.084)\end{array}$ & $\begin{array}{l}0.177^{* * *} \\
(0.059)\end{array}$ & $\begin{array}{c}0.011 \\
(0.008)\end{array}$ \\
\hline Transmigrant village $(=1)$ & & $\begin{array}{l}-0.035 \\
(0.077)\end{array}$ & $\begin{array}{l}-0.053 \\
(0.078)\end{array}$ & $\begin{array}{l}-0.051 \\
(0.088)\end{array}$ & $\begin{array}{c}0.091 \\
(0.099)\end{array}$ & $\begin{array}{l}-0.149 \\
(0.103)\end{array}$ & $\begin{array}{l}-0.040 \\
(0.075)\end{array}$ & $\begin{array}{l}-0.003 \\
(0.007)\end{array}$ \\
\hline $\begin{array}{l}\text { Distance to the nearest market } \\
(\mathrm{km})\end{array}$ & & $\begin{array}{l}-0.002 \\
(0.003)\end{array}$ & $\begin{array}{l}-0.002 \\
(0.003)\end{array}$ & $\begin{array}{l}-0.003 \\
(0.003)\end{array}$ & $\begin{array}{l}-0.003 \\
(0.003)\end{array}$ & $\begin{array}{l}-0.001 \\
(0.005)\end{array}$ & $\begin{array}{l}-0.003 \\
(0.003)\end{array}$ & $\begin{array}{l}-1.5 \mathrm{e}-04 \\
(2.4 \mathrm{e}-04)\end{array}$ \\
\hline Access to formal credit $(=1)$ & & $\begin{array}{c}0.069 \\
(0.047)\end{array}$ & $\begin{array}{l}0.093^{* *} \\
(0.045)\end{array}$ & $\begin{array}{l}0.089^{*} \\
(0.049)\end{array}$ & $\begin{array}{l}0.089 \\
(0.059)\end{array}$ & $\begin{array}{c}0.075 \\
(0.058)\end{array}$ & $\begin{array}{c}0.064 \\
(0.048)\end{array}$ & $\begin{array}{l}0.005 \\
(0.005)\end{array}$ \\
\hline $\begin{array}{l}\text { Share of land with systematic } \\
\text { land title (ratio) }\end{array}$ & & $\begin{array}{l}0.180^{* * * *} \\
(0.065)\end{array}$ & $\begin{array}{l}0.187^{* * *} \\
(0.067)\end{array}$ & $\begin{array}{l}0.136^{*} \\
(0.071)\end{array}$ & $\begin{array}{c}0.045 \\
(0.090)\end{array}$ & $\begin{array}{l}0.292^{* * *} \\
(0.100)\end{array}$ & $\begin{array}{l}0.194^{* * * *} \\
(0.065)\end{array}$ & $\begin{array}{c}0.014 \\
(0.011)\end{array}$ \\
\hline Constant & $\begin{array}{l}8.893^{* * *} \\
(0.119) \\
\end{array}$ & $\begin{array}{l}7.545^{* * *} \\
(7.545) \\
\end{array}$ & $\begin{array}{l}7.609^{* * *} \\
(7.609) \\
\end{array}$ & $\begin{array}{l}7.265^{* * *} \\
(7.265) \\
\end{array}$ & $\begin{array}{l}7.764^{* * *} \\
(7.764) \\
\end{array}$ & $\begin{array}{l}7.188^{* * *} \\
(7.188) \\
\end{array}$ & & \\
\hline $\mathrm{F}, \chi^{2}, \mathrm{R}^{2}$ & 4.843 & 599.3 & 627.3 & 355.5 & 24.90 & 17.35 & 0.2523 & 0.2523 \\
\hline Observations & 1278 & 1361 & 1361 & 1361 & 679 & 682 & 1274 & 1274 \\
\hline
\end{tabular}


Table A11: Impact of oil palm cultivation on food consumption expenditures

\begin{tabular}{|c|c|c|c|c|c|c|c|c|}
\hline & $\begin{array}{c}\text { (1) } \\
\text { Fixed effects }\end{array}$ & $\begin{array}{c}(2) \\
\text { Random effects: } \\
\text { Full model }\end{array}$ & $\begin{array}{c}(3) \\
\text { Random Effects: } \\
\text { Without own } \\
\text { business } \\
\end{array}$ & $\begin{array}{c}(4) \\
\text { Random Effects: } \\
\text { Without farm } \\
\text { size } \\
\end{array}$ & $\begin{array}{c}\text { (5) } \\
\text { Ordinary least } \\
\text { squares } 2012\end{array}$ & $\begin{array}{c}6) \\
\text { Ordinary least } \\
\text { squares } 2015\end{array}$ & $\begin{array}{c}(7) \\
\text { Spatial Durbin } \\
\text { model: } \\
\text { Direct effect } \\
\end{array}$ & $\begin{array}{c}(8) \\
\text { Spatial Durbin } \\
\text { model: Indirect } \\
\text { effect } \\
\end{array}$ \\
\hline Oil palm cultivation (dummy) & $\begin{array}{l}0.077 \\
(0.107)\end{array}$ & $\begin{array}{l}0.101^{* * * *} \\
(0.030)\end{array}$ & $\begin{array}{l}0.110^{* * *} \\
(0.031)\end{array}$ & $\begin{array}{l}0.139^{* * *} \\
(0.032)\end{array}$ & $\begin{array}{l}0.076^{* *} \\
(0.037)\end{array}$ & $\begin{array}{l}0.134^{* * *} \\
(0.045)\end{array}$ & $\begin{array}{l}0.089^{* * *} \\
(0.031)\end{array}$ & $\begin{array}{l}0.007 \\
(0.043)\end{array}$ \\
\hline Total farm size (ha) & $\begin{array}{c}0.002 \\
(0.011)\end{array}$ & $\begin{array}{l}0.017^{* * *} \\
(0.004)\end{array}$ & $\begin{array}{l}0.018^{* * * *} \\
(0.004)\end{array}$ & & $\begin{array}{l}0.020^{* * * *} \\
(0.005)\end{array}$ & $\begin{array}{l}0.013^{* * * *} \\
(0.004)\end{array}$ & $\begin{array}{l}0.016^{* * * *} \\
(0.004)\end{array}$ & $\begin{array}{l}0.002^{*} \\
(0.001)\end{array}$ \\
\hline Number of adults in household & $\begin{array}{l}-0.044^{* *} \\
(0.019)\end{array}$ & $\begin{array}{l}-0.042^{* * *} \\
(0.010)\end{array}$ & $\begin{array}{l}-0.036^{* * *} \\
(0.010)\end{array}$ & $\begin{array}{l}-0.037^{* * *} \\
(0.010)\end{array}$ & $\begin{array}{l}-0.031^{* *} \\
(0.014)\end{array}$ & $\begin{array}{l}-0.053^{* * *} \\
(0.014)\end{array}$ & $\begin{array}{l}-0.041^{* * *} \\
(0.009)\end{array}$ & $\begin{array}{l}-0.005^{*} \\
(0.003)\end{array}$ \\
\hline Own business $(=1)$ & $\begin{array}{l}0.159^{* * *} \\
(0.051)\end{array}$ & $\begin{array}{l}0.160^{* * *} \\
(0.032)\end{array}$ & & $\begin{array}{l}0.170^{* * *} \\
(0.033)\end{array}$ & $\begin{array}{l}0.203^{* * *} \\
(0.052)\end{array}$ & $\begin{array}{l}0.121^{* *} \\
(0.051)\end{array}$ & $\begin{array}{l}0.155^{* * *} \\
(0.030)\end{array}$ & $\begin{array}{l}0.018^{*} \\
(0.009)\end{array}$ \\
\hline Employment (=1) & $\begin{array}{c}0.044 \\
(0.038)\end{array}$ & $\begin{array}{c}0.020 \\
(0.021)\end{array}$ & $\begin{array}{l}-0.001 \\
(0.021)\end{array}$ & $\begin{array}{l}-0.007 \\
(0.023)\end{array}$ & $\begin{array}{c}0.038 \\
(0.034)\end{array}$ & $\begin{array}{l}-0.021 \\
(0.031)\end{array}$ & $\begin{array}{c}0.011 \\
(0.020)\end{array}$ & $\begin{array}{c}0.001 \\
(0.003)\end{array}$ \\
\hline $\begin{array}{l}\text { Wave (dummy; } 0=2012 ; 1= \\
\text { 2015) }\end{array}$ & $\begin{array}{l}0.155^{* * * *} \\
(0.026)\end{array}$ & $\begin{array}{l}0.148^{* * * * *} \\
(0.034)\end{array}$ & $\begin{array}{l}0.136^{* * * *} \\
(0.033)\end{array}$ & $\begin{array}{l}0.149^{* * * * *} \\
(0.035)\end{array}$ & & & $\begin{array}{l}0.133^{* * * *} \\
(0.030)\end{array}$ & $\begin{array}{l}0.015^{* *} \\
(0.008)\end{array}$ \\
\hline Female headed household $(=1)$ & & $\begin{array}{l}-0.124^{* * * *} \\
(0.040)\end{array}$ & $\begin{array}{l}-0.127^{* * * *} \\
(0.037)\end{array}$ & $\begin{array}{l}-0.129^{* * * * *} \\
(0.039)\end{array}$ & $\begin{array}{l}-0.160^{* *} \\
(0.055)\end{array}$ & $\begin{array}{l}-0.086 \\
(0.056)\end{array}$ & $\begin{array}{l}-0.126^{* * * *} \\
(0.046)\end{array}$ & $\begin{array}{l}-0.016 \\
(0.011)\end{array}$ \\
\hline $\begin{array}{l}\text { Age of household head } \\
\text { (Years) }\end{array}$ & & $\begin{array}{l}-0.011 \\
(0.008)\end{array}$ & $\begin{array}{l}-0.011 \\
(0.008)\end{array}$ & $\begin{array}{l}-0.006 \\
(0.008)\end{array}$ & $\begin{array}{l}-0.021^{* *} \\
(0.009)\end{array}$ & $\begin{array}{c}0.002 \\
(0.010)\end{array}$ & $\begin{array}{l}-0.013^{*} \\
(0.008)\end{array}$ & $\begin{array}{l}-0.002 \\
(0.001)\end{array}$ \\
\hline $\begin{array}{l}\text { Age of household head } \\
\text { squared }\end{array}$ & & $\begin{array}{c}1.2 \mathrm{e}-04 \\
(7.7 \mathrm{e}-05)\end{array}$ & $\begin{array}{l}1.2 \mathrm{e}-04 \\
(7.6 \mathrm{e}-05)\end{array}$ & $\begin{array}{c}8.3 \mathrm{e}-05 \\
(8.0 \mathrm{e}-05)\end{array}$ & $\begin{array}{l}2.3 \mathrm{e}-04^{* *} \\
(9.3 \mathrm{e}-05)\end{array}$ & $\begin{array}{l}-1.4 \mathrm{e}-05 \\
(1.0 \mathrm{e}-04)\end{array}$ & $\begin{array}{l}1.5 \mathrm{e}-04^{*} \\
(8.0 \mathrm{e}-05)\end{array}$ & $\begin{array}{l}1.9 \mathrm{e}-05 \\
(1.5 \mathrm{e}-05)\end{array}$ \\
\hline Education (years of schooling) & & $\begin{array}{l}0.014^{* * *} \\
(0.004)\end{array}$ & $\begin{array}{l}0.014^{* * *} \\
(0.004)\end{array}$ & $\begin{array}{l}0.017^{* * * *} \\
(0.004)\end{array}$ & $\begin{array}{l}0.013^{* *} \\
(0.005)\end{array}$ & $\begin{array}{l}0.017^{* * * *} \\
(0.006)\end{array}$ & $\begin{array}{l}0.015^{* * *} \\
(0.004)\end{array}$ & $\begin{array}{l}0.002^{*} \\
(0.001)\end{array}$ \\
\hline Non-random village $(=1)$ & & $\begin{array}{l}0.128^{* * * *} \\
(0.041)\end{array}$ & $\begin{array}{l}0.128^{* * *} \\
(0.038)\end{array}$ & $\begin{array}{l}0.161^{* * * *} \\
(0.046)\end{array}$ & $\begin{array}{c}0.082 \\
(0.088)\end{array}$ & $\begin{array}{l}0.168^{* * * *} \\
(0.055)\end{array}$ & $\begin{array}{l}0.099^{* * * *} \\
(0.040)\end{array}$ & $\begin{array}{l}0.011^{*} \\
(0.007)\end{array}$ \\
\hline Spontaneous migrant $(=1)$ & & $\begin{array}{c}0.008 \\
(0.031)\end{array}$ & $\begin{array}{c}0.010 \\
(0.031)\end{array}$ & $\begin{array}{c}0.007 \\
(0.033)\end{array}$ & $\begin{array}{c}0.018 \\
(0.043)\end{array}$ & $\begin{array}{c}0.012 \\
(0.039)\end{array}$ & $\begin{array}{c}0.020 \\
(0.027)\end{array}$ & $\begin{array}{c}0.002 \\
(0.004)\end{array}$ \\
\hline Transmigrant village $(=1)$ & & $\begin{array}{l}0.129^{* * * * *} \\
(0.040)\end{array}$ & $\begin{array}{l}0.115^{* * * *} \\
(0.044)\end{array}$ & $\begin{array}{l}0.123^{* * * *} \\
(0.045)\end{array}$ & $\begin{array}{l}0.104^{* *} \\
(0.046)\end{array}$ & $\begin{array}{l}0.143^{* *} \\
(0.068)\end{array}$ & $\begin{array}{l}0.112^{* * * *} \\
(0.045)\end{array}$ & $\begin{array}{l}0.012^{*} \\
(0.007)\end{array}$ \\
\hline $\begin{array}{l}\text { Distance to the nearest market } \\
(\mathrm{km})\end{array}$ & & $\begin{array}{l}-0.001 \\
(0.002)\end{array}$ & $\begin{array}{l}-0.001 \\
(0.002)\end{array}$ & $\begin{array}{l}-0.001 \\
(0.002)\end{array}$ & $\begin{array}{l}-0.003 \\
(0.002)\end{array}$ & $\begin{array}{c}0.003 \\
(0.002)\end{array}$ & $\begin{array}{l}-4.5 \mathrm{e}-04 \\
(0.001)\end{array}$ & $\begin{array}{l}-7.3 \mathrm{e}-05 \\
(1.9 \mathrm{e}-04)\end{array}$ \\
\hline Access to formal credit $(=1)$ & & $\begin{array}{c}0.037 \\
(0.032)\end{array}$ & $\begin{array}{l}0.053^{*} \\
(0.031)\end{array}$ & $\begin{array}{l}0.045 \\
(0.032)\end{array}$ & $\begin{array}{l}-0.007 \\
(0.035)\end{array}$ & $\begin{array}{l}0.071^{*} \\
(0.041)\end{array}$ & $\begin{array}{l}0.048 \\
(0.031)\end{array}$ & $\begin{array}{l}0.006 \\
(0.005)\end{array}$ \\
\hline $\begin{array}{l}\text { Share of land with systematic } \\
\text { land title (ratio) }\end{array}$ & & $\begin{array}{l}-0.017 \\
(0.047)\end{array}$ & $\begin{array}{l}-0.014 \\
(0.049)\end{array}$ & $\begin{array}{l}-0.034 \\
(0.051)\end{array}$ & $\begin{array}{c}0.022 \\
(0.054)\end{array}$ & $\begin{array}{l}-0.059 \\
(0.059)\end{array}$ & $\begin{array}{l}-0.030 \\
(0.049)\end{array}$ & $\begin{array}{l}-0.003 \\
(0.007)\end{array}$ \\
\hline Constant & $\begin{array}{l}9.150^{* * *} \\
(0.076)\end{array}$ & $\begin{array}{l}9.160^{* * * *} \\
(9.160)\end{array}$ & $\begin{array}{l}9.199^{* * * *} \\
(9.199)\end{array}$ & $\begin{array}{l}9.055^{* * * *} \\
(9.055)\end{array}$ & $\begin{array}{l}9.485^{* * * *} \\
(9.485) \\
\end{array}$ & $\begin{array}{l}8.897^{* * * *} \\
(8.897) \\
\end{array}$ & & \\
\hline $\mathrm{F}, \chi^{2}, \mathrm{R}^{2}$ & 8.809 & 276.2 & 253.2 & 268.8 & 4.716 & 17.03 & 0.1668 & 0.1668 \\
\hline Observations & 1278 & 1361 & 1361 & 1361 & 679 & 682 & 1274 & 1274 \\
\hline
\end{tabular}




\section{Why does a labor-saving technology decrease fertility rates? Evidence from the oil palm boom in Indonesia ${ }^{11}$}

\subsection{Introduction}

There are many good reasons why reducing fertility is important. At the individual level, the health burden for women (including the risk of dying in childbed) as well as their socioeconomic wellbeing are directly associated with fertility declines (Chen et al. 1974; Campbell \& Graham 2006; Miller 2010). At the macroeconomic level, low fertility rates are often associated with higher incomes and more generally with higher and more sustained economic growth (Barro 1991; Lee \& Mason, Andrew 2006). Globally, population growth has been identified as an important factor contributing to environmental degradation and global warming (Bongaarts 1992; Dietz \& Rosa 1997; York et al. 2003; Luck 2007).

Different theories exist about what triggers decreasing fertility rates. Technological change is generally seen as one key driver of the historical fertility transition in the US and Europe (Galor \& Weil 2000; Guinnane 2011). Galor \& Weil (2000) argue in their theoretical model that technological change increases returns to education which leads to a substitution away from child quantity to child quality. The substitution effect is built on Becker's quantity-quality model (Becker \& Lewis 1973; Becker 1981). The linkage between increasing returns to education and decreasing fertility was mostly backed up by empirical findings (Bleakley \& Lange 2009; Becker et al. 2010; Fernihough 2017), although the results are not unambiguous (Black et al. 2005). Moreover, decreasing compatibility of work and child rearing (Rindfuss \& Brewster 1996) and diminishing value of child labor (Doepke 2004) are often cited as important transmission mechanisms for the linkage between technological change and fertility reduction. Only few papers have studied the full range of transmission mechanism regarding the effect of new technologies on fertility. For the fertility transition in the US, Wanamaker (2012) argues that industrialization led to fertility reductions in South Carolina between 1880 and 1900 due to

\footnotetext{
11 This essay is co-authored by Esther Gehrke. CK developed the research idea, reviewed the relevant literature, compiled the data, analyzed the data, and wrote the paper. EG contributed to the empirical design, data analysis, and writing of the paper.
} 
increasing maternal opportunity costs of time and a separation of migrant households from their extended family network. Ager et al. (2017) show for the American South in the same time period that households switching to manufacturing face higher opportunity costs of raising children since manufacturing work is generally less compatible with child rearing. Their results further suggest that rising returns to education and diminishing returns to child labor led parents to invest rather in child quality than child quantity.

In developing countries, empirical evidence on the relationship between technology and fertility is largely inexistent. For one, employment is still largely dominated by agriculture, and attempts to trigger industrialization processes often failed, limiting potential effects on fertility. Moreover, new technologies such as mechanization in agriculture are often concentrated on large farms, restricting direct income effects to a rather small elite. Only few studies have looked at the relationship between mechanization and fertility in lowincome settings, or at the mechanisms underlying this relationship. Rosenzweig \& Evenson (1977) and Levy (1985) are notable exceptions. However, these two studies concentrate entirely on changes in the demand for child labor and its effects on fertility.

This essay explores the effect of a different technology - the expansion of oil palm in Indonesia. ${ }^{12} \mathrm{We}$ argue that oil palm is rather unique since it is - similarly to mechanization - labor-saving compared to alternative crops in the region, and can free up substantial amounts of labor from agriculture (Rist et al. 2010; Euler et al. 2017). Moreover, it affects not only large-scale farms but also smallholder farmers. Unlike factor-neutral technologies that only raise productivity and thus income, we hypothesize that the labor savings induced by the expansion of oil palm play an important role in determining fertility decisions. Our research question is therefore if and through which mechanisms a labor saving technology such as oil palm affects fertility decisions in a developing country context.

We use Becker's quantity-quality model (henceforth Q-Q model) to identify different causal mechanism through which the expansion of oil palm could affect fertility rates. Our conceptual framework highlights five main mechanisms. The first mechanism is an income effect. While increases in income could generally increase the demand for children, it is generally assumed that the income elasticity of child quality is greater than the income elasticity of child quantity, thereby reducing fertility via a substitution effect. The second

\footnotetext{
${ }^{12}$ Although oil palm identifies as a technology only in a wider sense, we use this term to emphasize the changes in factor productivity and its comparability with mechanization.
} 
mechanism is an effect on the price of child quantity via a reduction in child labor. The third mechanism is also related to the price of child quantity: we expect the expansion of oil palm to affect maternal opportunity costs of time. The fourth and fifth mechanisms relate to the price of child quality: returns to education could rise, and infrastructure development could reduce the price of investing in child quality. We also discuss three alternative mechanisms that are not in line with the Q-Q model: female empowerment, migration patterns and child mortality.

In our empirical analysis, we focus on the oil palm expansion in Indonesia since the mid-1990s and explore a large set of different data sources. We use the National Socioeconomic Survey (SUSENAS) to assess changes in fertility, measured as the total number of children born per woman. Changes in wages, labor supply and sector of work are observed in the Indonesian Labor Force Survey (SAKERNAS). For the oil palm expansion, we rely on administrative data gathered by the Indonesian government, the Tree Crop Statistics, a data source which is also used to analyze the effects of oil palm on poverty rates (Edwards 2017). To complement this data source, we use land-use data from the Village Potential Statistics (PODES), which was collected in 1993 and 2003. Finally, we use Census data, the Demographic and Health Survey (DHS), as well as different administrative data sources to explore a number of causal mechanisms.

Our identification strategy builds on the fact that agro-ecological characteristics affect a regency's suitability for oil palm cultivation. ${ }^{13}$ Similarly to Duflo \& Pande (2007), we exploit two sources of variation in a fixed effects instrumental variables (IV) approach: First, we explore differences across space in terms of the maximum agro-climatically attainable yield for oil palm from the Global Agro-Ecological Zones (GAEZ) data. Second, we explore differences in the national expansion of oil palm area across time. The national expansion is used as a proxy for the development in global demand for oil palm. Combining these two sources of variation, we instrument regency-level oil palm area by its predicted level if the expansion were entirely driven by productivity concerns, that is, if oil palm was more quickly introduced in areas that are better suited to oil palm cultivation and only subsequently to less well suited areas.

\footnotetext{
${ }^{13}$ In Indonesia provinces are the highest tier of the local government. At the next level provinces are divided into regencies (kabupaten) and city districts (kotas). Since the decentralization in 2001, regencies (and city districts) are mainly responsible for providing public services.
} 
Using this instrumental variables approach, we find consistently negative effects of the oil palm expansion on fertility. These results are robust to controlling for island-year fixed effects and differential time trends between regencies with different initial characteristics, such as fertility, share of agricultural employment in total employment, agricultural wages and electrification. The results are also robust to using different measures for the oil palm expansion and for fertility, to using different time periods and to using a number of alternative specifications.

Our results on the different transmission mechanisms suggest that the bulk of the negative effect can be explained by an income effect at the household level, as well as by the local economic development that was induced by the oil palm expansion. Oil palm expansion increased income at the household level, which induced a substitution away from child quantity to child quality. The income growth also triggered broader local economic development, leading to a growing non-agricultural sector and increasing returns to education. This induced parents to substitute child quality for child quantity, as well as women to invest more into their own education, thus reducing their fertility. We conclude that labor saving technologies in agriculture can reduce (rather than increase) fertility as long as income gains are shared by the majority of the population and high enough to trigger local economic development.

Our results contribute to two different strands of literature. We add to the literature on the role of technology in determining fertility choices (Rosenzweig \& Evenson 1977; Levy 1985; Wanamaker 2012; Ager et al. 2017) by showing the impact and transmission mechanisms of a labor-saving agricultural technology, which unlike mechanization is also rapidly adopted by smallholder farmers. ${ }^{14} \mathrm{We}$ also contribute to the growing literature on the effects of oil palm in Indonesia. Previous research has documented negative environmental effects such as a drastic loss of biodiversity (Wilcove \& Koh 2010; Clough et al. 2016), reduction of water resources (Merten et al. 2016) and increased carbon emissions (Burney et al. 2010), but also social impacts such as land conflicts (Obidzinski et al. 2012). However, the oil palm expansion also seems to have led to significant economic gains such as poverty reduction and increased welfare of smallholder farmers (Edwards

\footnotetext{
${ }^{14}$ Our research differs substantially from research using an exogenous shock on for example price of child quality (Bleakley \& Lange 2009; Becker et al. 2010) or quantity (Black et al. 2005) to confirm model predictions, since we focus on a technology with a wide range of potential effects. To test these effects a broad set of data is necessary. The data demands might also explain why this literature is rather limited yet.
} 
2017; Krishna et al. 2017a). To the best of our knowledge, our study is the first to address the demographic effects of the oil palm boom in Indonesia.

The remainder of this essay is structured as follows: In Section 4.2, we provide background information on the oil palm expansion in Indonesia and present evidence on the factor productivity of oil palm relative to alternative crops. Section 4.3 presents the conceptual framework. In Section 4.4, we introduce the different data sources to test our hypotheses. Our estimation strategy is presented in Section 4.5. Section 4.6 reports main results, as well as several robustness checks and an analysis of the transmission mechanisms. Conclusions are presented in Section 4.7.

\subsection{Background: Oil palm in Indonesia}

Global palm oil production rose steeply by 300\% between 1990 and 2010 with Indonesia emerging as the world's largest producer around 2009 (Byerlee et al. 2017). Although oil palm has been cultivated in Indonesia since the 1930s (Verheye 2010), the expansion only accelerated in the 1970s with the central government supporting the establishment of large-scale plantations in the outer islands. In so-called Nucleus Estate and Smallholder (NES) schemes, large estates surrounded by smallholder plantations were built up, tying the smallholder farmers via contract farming to the estates. In sparsely populated regions, laborers and farmers were often recruited from the central islands such as Java. With the decentralization process starting in 1998, market liberalizations and the subsequent decrease in governmental support for NES schemes, more independent adopters emerged and contractual ties between contract farmers and companies loosened (Euler et al. 2016b). Although large private estates still dominate oil palm cultivation in Indonesia, smallholders cultivated roughly $40 \%$ of the total oil palm area in the country in 2016 (see Figure A1 in the Appendix).

Positive welfare gains of oil palm have been documented for smallholders by Euler et al. (2017) and Rist et al. (2010). These welfare gains seem to be driven by the low labor intensity of oil palm compared to alternative crops such as rubber and rice, which allows 
for farm expansion and additional off-farm work. ${ }^{15}$ In order to provide more detailed evidence on the factor productivity of oil palm compared to other crops, we explore farm household data collected in Jambi Province (Sumatra) in 2012 and $2015 .^{16}$ The data contains detailed plot input and output information for farmers involved in the cultivation of oil palm and rubber. ${ }^{17}$ In addition, we cite evidence from the literature concerning rice cultivation.

Table 6: Labor and land productivity of oil palm and rubber

\begin{tabular}{|c|c|c|c|c|}
\hline & \multicolumn{2}{|r|}{ Oil palm } & \multicolumn{2}{|r|}{ Rubber } \\
\hline & Obs. & Mean (Std. dev.) & Obs. & Mean (Std. dev.) \\
\hline $\begin{array}{l}\text { Labor productivity } \\
\text { [000 IDR/hour] }\end{array}$ & 437 & $88.066^{* * * *}(110.483)$ & 967 & $22.752(25.192)$ \\
\hline $\begin{array}{l}\text { Land productivity } \\
\text { [000 IDR/ha/year] }\end{array}$ & 437 & $16333.14^{* * *}(11889.91)$ & 967 & $18157.01(12108.73)$ \\
\hline $\begin{array}{l}\text { Capital input } \\
\text { [000 IDR/ha/year] }\end{array}$ & 439 & $2653.118^{* * * *}(2662.383)$ & 973 & 651.995 (1021.978) \\
\hline $\begin{array}{l}\text { Female labor input } \\
\text { [Hours/ha/year] }\end{array}$ & 439 & $25.764^{* * *}(65.35)$ & 973 & $313.761(471.624)$ \\
\hline $\begin{array}{l}\text { Male labor input } \\
\text { [Hours/ha/year] }\end{array}$ & 439 & $237.696^{* * *}(211.089)$ & 973 & 854.687 (997.983) \\
\hline $\begin{array}{l}\text { Female wages } \\
\text { [000 IDR/hour] }\end{array}$ & 17 & $12.442(11.353)$ & 27 & $10.437(1.751)$ \\
\hline $\begin{array}{l}\text { Male wages } \\
\text { [000 IDR/hour] }\end{array}$ & 167 & $18.227^{* * *}(17.222)$ & 319 & $14.411(15.580)$ \\
\hline
\end{tabular}

Notes: Statistical significant difference between crops was tested using a t-test. Unproductive plots were excluded and tree age restricted to productive age from 5 to 25 years (except for wage data). For the male wage data two outliers with more than 10 times the average wage were excluded. Hours worked include family as well wage labor. All monetary values are in constant 2012 values.

Plot level estimates in Table 6 show that labor productivity per hour is significantly higher in oil palm compared to rubber. This is also reflected in higher wages paid in both activities: wages are higher for men in oil palm than in rubber cultivation. However, the

${ }^{15}$ At the national level, PODES data suggests that rubber and rice are the main alternatives to oil palm cultivation. In $53 \%$ of the villages where oil palm was the first or second most important planation crop in 1993, rubber was either the first or second most important crop. In 63\% of the oil palm villages, rice was also mentioned as important food crop. More recent data from Sumatra also shows that oil palm is increasingly replacing rubber as the dominant plantation crop, and to a lesser extent rice (Feintrenie et al. 2010; Gatto et al. 2015; Euler et al. 2016b). Evidence from Kalimantan also suggests that oil palm mainly replaced laborintensive crops such as rubber and rattan (Belcher et al. 2004).

${ }^{16}$ We use this data, because no nationally representative micro data with detailed input and output information is available in Indonesia.

17 A multi-stage sampling framework was used to obtain a representative sample of 700 local farm households in 45 villages in the tropical lowlands of Jambi. For more details on the sampling framework, see Krishna et al. (2017b). 
difference in wages is smaller for women and not significant. The finding that labor productivity is higher in oil palm relative to rubber is generally confirmed in the literature (Rist et al. 2010; Euler et al. 2017). The labor productivity gap between oil palm and rice is even larger with 47.33 to 2.27 \$ per men's labor day (Rist et al. 2010). In contrast, land productivity of oil palm is lower than in rubber cultivation. This is not true in comparison to rice: Rist et al. (2010) report a significantly lower land productivity for inundated rice compared to rubber and oil palm $(2846.36 \$$ per ha for oil palm and $264.61 \$$ per ha for rice). Switching from a food crop such as rice to a cash crop thus increases welfare by raising labor (and partly land) productivity.

Our data also suggests that the gains in labor productivity largely translate into a reduction of labor inputs. As can be seen in Table 6, labor hours per hectare of women and men are substantially lower in the cultivation of oil palm than in the cultivation of rubber. Male labor hours are by $72 \%$ lower in oil palm compared to rubber. Female labor hours are even by $92 \%$ lower. The low labor input in oil palm in particular for women was also reported for large-scale plantations, and is mainly due to the tasks associated with oil palm cultivation. While rubber tapping is often done every day or every two days and does not necessitate a lot of physical strength, oil palm harvesting is done a lot less frequently (on average every two weeks) and is mainly done by men. Women are mainly involved in the collection of loose oil palm fruits and maintenance work (Koczberski 2007; Li 2015). Typically, food crops such as rice also involve comparably more female labor than cash crop cultivation, and rice cultivation in Indonesia is no exception. Rice has a low labor productivity in general, and there is no evidence that female labor is more or less productive than male labor in rice cultivation (Feintrenie et al. 2010; Li 2015).

While welfare gains from oil palm cultivation among smallholder farmers seem to be driven by increases in labor productivity, expansion of farm land and the reallocation of working hours towards other sectors, these effects are expected to look quite differently for wage workers. The majority of palm oil is still produced by large private estates, which rely entirely on wage work, and also farm households employ significant amounts of wage labor (Euler et al. 2017). The wage labor is partly drawn from migrants, but evidence from Kalimantan suggests that local population groups that lack the financial means to establish their own plantations are also employed (Li 2015). Higher labor productivity in oil palm cultivation could increase wages. However, if land is scarce, the demand for agricultural 
labor might decrease and thus also decrease wages, or limit welfare gains to a very small group of farm workers. While the welfare effect of the oil palm expansion for wage workers is found to be positive on average (Edwards 2017), this might mask substantial regional heterogeneity.

In addition to the welfare gains documented above, one important aspect of oil palm that might drive broader economic development is the need to process fresh oil palm fruit bunches shortly after harvest in palm oil mills. This necessitates improved road infrastructure in order to quickly transport the fresh fruit bunches from the producer to the mill and reliable access to electricity to run palm-oil mills. Finally, some high-skilled labor is needed to operate the mills (Edwards 2017). In general, welfare gains and infrastructure development might have contributed to broader local economic development through increased demand and reduced costs in transportation and production, thereby increasing wages and creating job opportunities outside the oil palm sector.

\subsection{Conceptual framework}

The previous Section highlighted a range of mechanisms through which oil palm could raise household welfare. This Section builds on the Q-Q model developed in Becker and Lewis (1973) to derive testable predictions of the effect of the oil palm expansion on fertility. In particular, we seek to highlight the mechanisms through which this effect might be operating. Possible extensions of the Q-Q model are discussed in Section 4.3.2.

\subsubsection{A simple model on oil palm expansion and demand for children}

We follow Becker and Lewis (1973) in assuming a household utility function of the form $U(n, q, Z)$ with $q$ being the quality of each child, $n$ the number of children and $Z$ other commodities. This utility function is maximized subject to the following budget constraint:

$$
p_{n} n+p_{q} q+p_{c} n q+\pi_{Z} Z=I
$$


In this budget constraint, $I$ is the full income of the household. $p_{n}$ is the cost of having one additional child, thus the opportunity cost of time of pregnancy and individual child rearing, and all other monetary costs of having children that are largely independent of child quality. The net cost of having children $p_{n}$ falls if children contribute to farm income, and falls with increasing costs of avoiding pregnancies. $p_{q}$ is the cost of child quality which is independent of number of children, such as reusable school books and clothing, or accessing information on the school system. $p_{c}$ is the cost of augmenting the quality of any child, such as school fees, and the respective price of other commodities is $\pi_{Z}$.

We now consider how the adoption of a new agricultural technology, such as oil palm, affects the demand for children. We assume that the crop is adopted because it increases farm income and do not model the agricultural production function explicitly. Furthermore, we assume that the positive income effect dominates in all population segments. Given the effects on labor productivity, income and infrastructure development cited above, we expect oil palm to affect fertility mainly through five mechanisms, which will be discussed in the following.

Income. If oil palm raises farm income and potentially also income from agricultural employment, households can invest this additional income in increasing the number of children, in increasing child quality or both. The number of children could hence decrease or increase. However, following Becker and Lewis (1973), we assume that the income elasticity of child quality is higher than the income elasticity of child quantity. Because increasing the quality of each child affects the shadow price of child quantity through the interaction term between quality and quantity, even a small increase in $q$ could have a large and negative effect on $n .^{18}$ This is why we generally expect the income effect on child quantity to be negative.

Child labor. A price effect of child quantity might stem from differences in the returns to child labor between oil palm and alternative crops. In many countries children generate income through family work or wage work, thereby offsetting some of their direct costs such as clothing and food. We are not aware of any detailed empirical analysis of child labor in oil palm cultivation. Anecdotal evidence suggests that children can be involved in picking up loose fruits, which fell off the main bunch during harvest, but also in more demanding work such as carrying harvest and other heavy loads (Koczberski 2007;

\footnotetext{
${ }^{18}$ See Becker (1981) or Becker \& Lewis (1973) for more details.
} 
Amnesty International 2016). However, harvesting and cutting of oil palm necessitates too much physical strength to involve child labor. Rubber and rice cultivation, in contrast, involve more family labor and theoretically also more child labor. We therefore expect that oil palm would rather decrease the returns to child labor. ${ }^{19}$ If returns to child labor fall, this increases the cost of child quantity $p_{n}$, thereby reducing the demand for children.

Maternal opportunity costs of time. The price of child quantity also shifts with changing maternal opportunity costs of time. As noted in the previous Section, oil palm cultivation is less labor intensive, and employs considerably less women than alternative crops. If female shadow wages in agriculture fall, women could either stop working or shift to other sectors. However, as the oil palm expansion might go hand-in-hand with income growth and local economic development, wages for women in other sectors than agriculture could even increase, thus drawing more women into the labor force and out of agriculture. This provides two possible scenarios: If female labor is not sufficiently demanded, we would expect that female labor force participation and female wages decrease. This implies a reduction of $p_{n}$ and ceteris paribus an increase in the demand for children. In the second scenario, the income gains in the agricultural sector spur broader economic development and while women still leave the agricultural sector, they now enter an increasingly more profitable non-agricultural sector. Then, we would expect $p_{n}$ to increase due to higher wages and women reallocating their time from child rearing to income earning activities. If the non-agricultural sector is less suitable to combine child rearing and income generation due to the distance between dwelling and workplace or less amenable working environment, we would expect additional increases in the opportunity costs of time and fertility reductions. Also, if local economic development creates more jobs in the high education sector, then the wage gap between low education and high education jobs might widen and returns to education increase. This might again affect maternal opportunity costs of time and thus the price of child quantity $p_{n}$, as we would expect that women reallocate their time away from child rearing to schooling.

Returns to children's education. Not only the price of child quantity, also the price of quality is expected to change with the oil palm expansion. Building on the second scenario mentioned above, we assume that higher returns to education not only increase maternal

\footnotetext{
${ }^{19}$ Note that the microdata presented in Section 2 do not provide information about child labor, which by 2012 was largely abolished. This does not imply that child labor did not play a more important role in the 1990s.
} 
opportunity costs of time, but also the returns to children's education. Investing into children's education is likely to pay off more if profitable jobs in the high education sector exist. If returns to education increase, parents are more likely to substitute child quality for quantity, thus decreasing their demand for children.

Infrastructure. Finally, the infrastructure created due to oil palm could reduce the price of child quality. Since fresh fruit bunches have to be brought to palm oil mills within two days to guarantee high quality oil, transportation infrastructure such as asphalt roads are in particular likely to be associated with the oil palm expansion. In addition, higher incomes can provide higher tax revenues for local governments, which can in turn lead to higher investments in health, education and transportation infrastructure. These investments would reduce the cost of accessing education, thereby decreasing the cost of investing in any child's quality. In the Q-Q model, a reduction in the price of child quality would increase investments in child quality, and through the interaction between quality and quantity, this would again decrease the demand for children.

\subsubsection{Alternative explanations}

While the Q-Q model highlights a range of important mechanisms, it imposes a set of assumptions which might not necessarily be true. For one, the assumption of a unitary household has been subject to a lot of debate recently. Furthermore, fertility might respond more strongly to social preferences rather than household choice. In the following, we will discuss three potential alternative explanations, which seem particularly relevant in the context of oil palm cultivation: Migration, child mortality and female empowerment.

Migration. The oil palm boom increased internal migration flows into oil palm cultivating areas through the transmigration program as well as through spontaneous migration (Euler et al. 2016b). In the very short term migrant families might have faced increasing opportunity costs of child rearing since the establishment of a new farm and household are labor intensive tasks. On the other hand, men are more likely to be involved in internal migration, increasing the share of women in sending regions (Sukamdi \& Mujahid 2015). This could have decreased fertility in sending regions compared to oil palm cultivating areas due to the decreasing likelihood of marriage. In the long run both these factors might be less important and childbearing patterns might depend more on the 
question if there was a selection of migrants by fertility preference and if migrants' fertility preferences differed from the local population (Kulu 2005).

Child mortality. An alternative mechanism could be that fertility decreases as more children survive (Kirk 1996). The idea is that families have a desired fertility with respect to the number of surviving children, and that this target can be achieved with lower overall fertility rates as child mortality decreases. Since oil palm expansion improved infrastructure and incomes, and this probably decreased child mortality, households might have simply adjusted the number of births but not the number of desired children.

Female empowerment. A substantial body of literature suggests that female bargaining power within the household increases as women earn their own income (Atkin 2009; Heath $\&$ Mobarak 2015). If women have per se lower fertility preferences than men, a reduction in fertility could stem from the fact that women leave the agricultural sector (and on-farm work) and start earning their own income over which they have higher control than farm income. The fertility reduction would then simply reflect the increased bargaining weight of women within the household that is associated with the oil palm expansion.

\subsection{Data}

We combine different datasets to assess the effect of the oil palm expansion on fertility and to analyze the underlying mechanisms. We merge all datasets at the regency level using 1993 boundaries. This was necessary due to Indonesia's decentralization process, which involved a continuous division of regencies over the past 20 years. A detailed list of all data sources can be found in Table A12 in the Appendix. Table 7 presents summary statistics.

Administrative data on the oil palm expansion at regency level is available since 1996. The Tree Crop Statistics are published annually by the Indonesian government (Ministry of Agriculture 2017), and can be accessed through the Indonesia Database for Policy and Economic Research (INDO-DAPOER) which is maintained by the World Bank (World Bank 2018a). ${ }^{20}$ The data provides information on the area under oil palm cultivation, and

\footnotetext{
${ }^{20}$ We update the database with more recent data from the Tree Crop Statistics to complete the time series until 2015. We do not have consistent data for oil palm expansion on regency level for 2016 and thus use 2015 data if necessary.
} 
distinguishes between four producer categories, smallholder, government estate, national private estate, and foreign private estate. However, time series at regency level dating back to the 1990s are only available for smallholder producers and not for private or government estates.

Table 7: Summary statistics (1996-2006-2016)

\begin{tabular}{|c|c|c|c|}
\hline & Obs. & Mean & SD \\
\hline \multicolumn{4}{|l|}{ For women 15-49: } \\
\hline Number of children born alive & 604159 & 1.875 & 1.927 \\
\hline Age & 604219 & 30.745 & 9.885 \\
\hline Age at first marriage & 447766 & 19.772 & 3.783 \\
\hline Ever married $(=1)$ & 604219 & 0.741 & 0.438 \\
\hline Working $(=1)$ & 604218 & 0.465 & 0.499 \\
\hline Working hours & 604219 & 16.042 & 21.239 \\
\hline Working in agriculture $(=1)$ & 604219 & 0.217 & 0.412 \\
\hline Working in family agriculture $(=1)$ & 604219 & 0.138 & 0.345 \\
\hline Working in service sector $(=1)$ & 604219 & 0.187 & 0.390 \\
\hline HH head self-employed in agri. $(=1)$ & 604219 & 0.385 & 0.487 \\
\hline HH head employed in agri. $(=1)$ & 604219 & 0.080 & 0.271 \\
\hline Monthly expenditures per cap. (IDR) & 604219 & 69899.75 & 65353.81 \\
\hline Share of food expenditures of total exp. & 604219 & 0.623 & 0.140 \\
\hline Living in rural area $(=1)$ & 604219 & 0.709 & 0.454 \\
\hline \multicolumn{4}{|l|}{ Educational attainment } \\
\hline Primary school $(=1)$ & 604219 & 0.364 & 0.481 \\
\hline Secondary junior school $(=1)$ & 604219 & 0.190 & 0.392 \\
\hline Secondary high school $(=1)$ & 604219 & 0.194 & 0.395 \\
\hline Tertiary schooling $(=1)$ & 604219 & 0.050 & 0.218 \\
\hline \multicolumn{4}{|l|}{ Regency level: } \\
\hline Share of smallholder OP area in regency & 626 & 0.009 & 0.025 \\
\hline Share of villages in regency with asphalt main road & 626 & 0.721 & 0.187 \\
\hline Share of villages in regency with kindergarten & 626 & 0.692 & 0.260 \\
\hline Share of villages in regency with primary school & 626 & 0.984 & 0.031 \\
\hline Share of villages in regency with junior high school & 626 & 0.511 & 0.164 \\
\hline Share of villages in regency with hospital & 626 & 0.040 & 0.039 \\
\hline Share of $\mathrm{HH}$ in regency with access to public grid & 626 & 0.638 & 0.254 \\
\hline Share of girls $12-14 y$ with primary degree & 626 & 0.568 & 0.111 \\
\hline Share of boys $12-14 y$ with primary degree & 626 & 0.523 & 0.115 \\
\hline Share of individuals ever migrated to regency & 569 & 0.084 & 0.112 \\
\hline Share of individuals migrated in last $5 y$ to regency & 569 & 0.045 & 0.029 \\
\hline \multicolumn{4}{|l|}{ Province level: } \\
\hline Female wage in non-agr. employment (IDR/hour) & 66 & 774.734 & 171.636 \\
\hline Female wage in agr. employment (IDR/hour) & 66 & 528.272 & 215.961 \\
\hline Male wage in non-agr. employment (IDR/hour) & 66 & 947.589 & 198.003 \\
\hline Male wage in agr. employment (IDR/hour) & 66 & 669.341 & 249.506 \\
\hline Child mortality (per 1000 births) & 66 & 14.526 & 9.050 \\
\hline
\end{tabular}

Notes: Data is available for 209 regencies. We miss data for one regency in 2016. For migration multiple regencies have missing data. Indonesia had 26 provinces in 1996. However, we do not have data for Maluku, Papua and Aceh and Jakarta is exclusively urban; we hence end up with 22 provinces. No data on child mortality and migration are available in 2016, we use data from 2012 and 2010 instead. All monetary values are in constant 1996 IDR. Exchange rate was at 2342 IDR/US\$ in 1996 (World Bank 2018b). 
As can be seen in Figure A1 in the Appendix, the expansion of oil palm over time in the smallholder sector is fairly parallel to the expansion in the private estates. Government estates are less important. Also, the expansion of the large-scale plantation sector and the smallholder sector are likely to correlate regionally, since the smallholder sector depends on access to palm oil mills which are often established within the large-scale plantations (Euler et al. 2016b). Figure 6 illustrates the expansion of smallholder oil palm area on the different islands of Indonesia. It shows a strong concentration of oil palm on Sumatra, but also the growing importance of Kalimantan and to some extent of Sulawesi.

Figure 6: Regional oil palm expansion of smallholders in Indonesia in 1996 and 2015
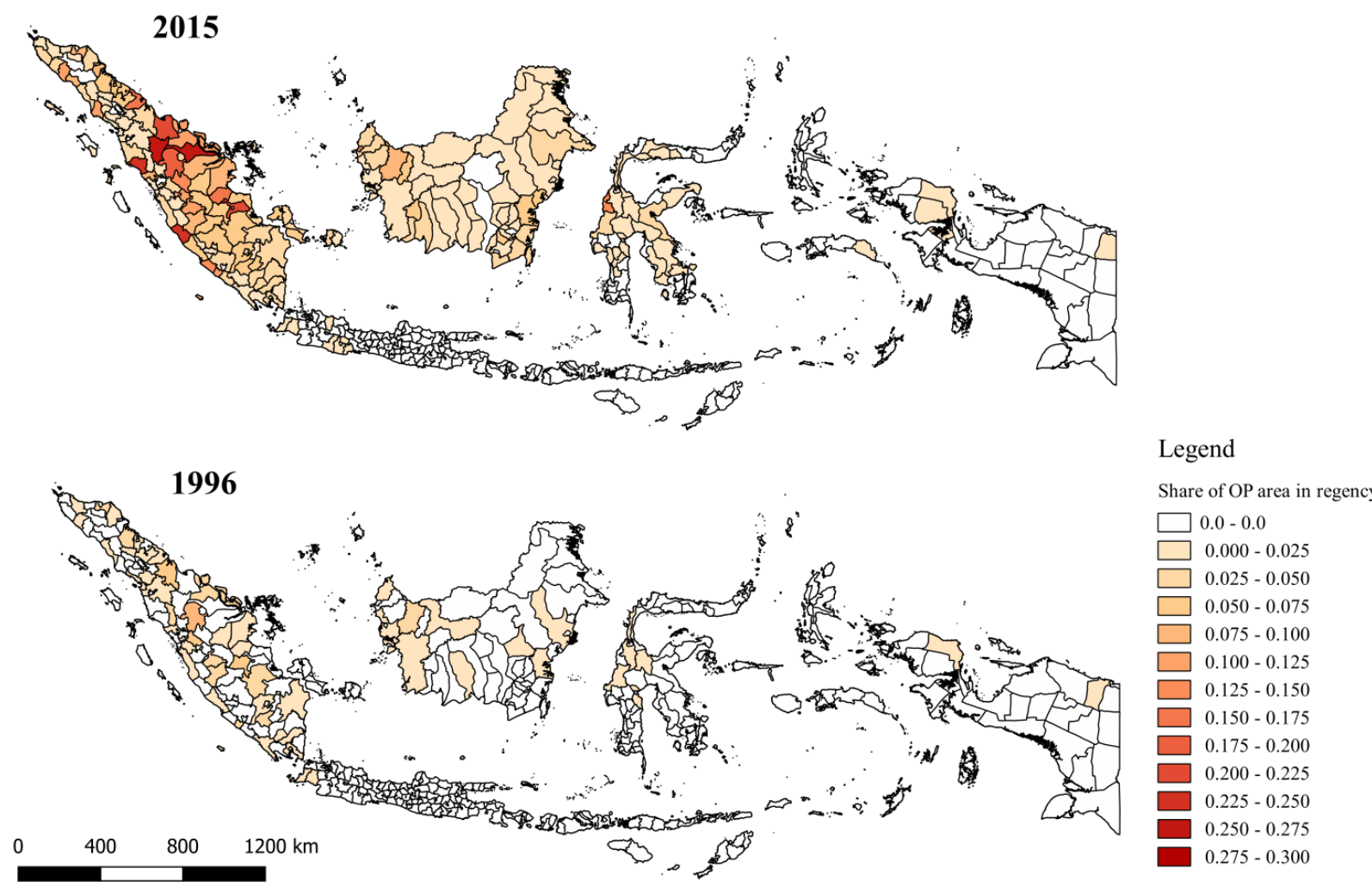

Source: Tree crop statistics, INDO-DAPOER.

The PODES - Indonesia's village census - provides the earliest data on oil palm expansion that is nationally representative and can be disaggregated by regency. The PODES data covers all villages and urban neighborhoods in Indonesia. It collects information on village-level land use in the years 1993 and 2003. Based on this information, we calculate the share of villages within a regency that cultivate oil palm. We 
use this variable for additional robustness checks. The PODES dataset also provides additional controls such as the share of villages with schools, hospitals and asphalt roads within a regency, as well as the share of households with access to the public electricity grid.

We use Indonesia's socioeconomic survey (SUSENAS) to construct individual fertility. ${ }^{21}$ The SUSENAS collects demographic and socioeconomic characteristics of individuals in annually repeated cross sections. Since 1993 the sample size increased to more than 200.000 households from formerly around 65.000 , being representative at the regency-level. The SUSENAS provides information on the number of all ever occurred live births per woman, including all women older than 10 years. We use this variable as our measure of fertility, and restrict the sample to women aged 15 to 49 . Our measure of fertility is not directly comparable to the total fertility rate (TFR). The TFR is the average number of children that would be born to every woman over her lifetime based on current age-specific fertility rates, assuming constant age-specific fertility rates over time and no premature deaths of women. Our measure of fertility, in contrast, has no reference period and is therefore sensitive to fertility changes that already happened in the past. These differences are also reflected in differential time trends: Between 1996 and 2016, fertility decreased from 2.11 to 1.70 children born per woman in Indonesia, while the TFR only fell from 2.6 to 2.4 in the same time period (World Bank 2018c). Figure 7 presents the fertility trends based on SUSENAS for different islands in Indonesia. ${ }^{22}$ The figure shows that the fertility rate decreased until 2005, stagnating in some islands in subsequent years. Additional variables derived from SUSENAS are age, age at marriage, education, consumption expenditure, labor supply and type of work. ${ }^{23}$

\footnotetext{
${ }^{21}$ The regencies of Papua, Aceh and the Maluku islands were dropped since data in these regions are not available for all years due to social unrest. Since oil palms are not cultivated within cities, we also exclude all city districts from the analysis.

${ }^{22}$ Although we speak of islands, these are the main islands of Sumatra, Kalimantan, Java and Bali and Sulawesi, including their adjacent islands. All islands which do not belong to these regions are included in the fifth category "other islands".

${ }^{23}$ We deflate all monetary values to 1996 values using the province-level poverty lines for rural and urban regions.
} 
Figure 7: Regional fertility trends in Indonesia

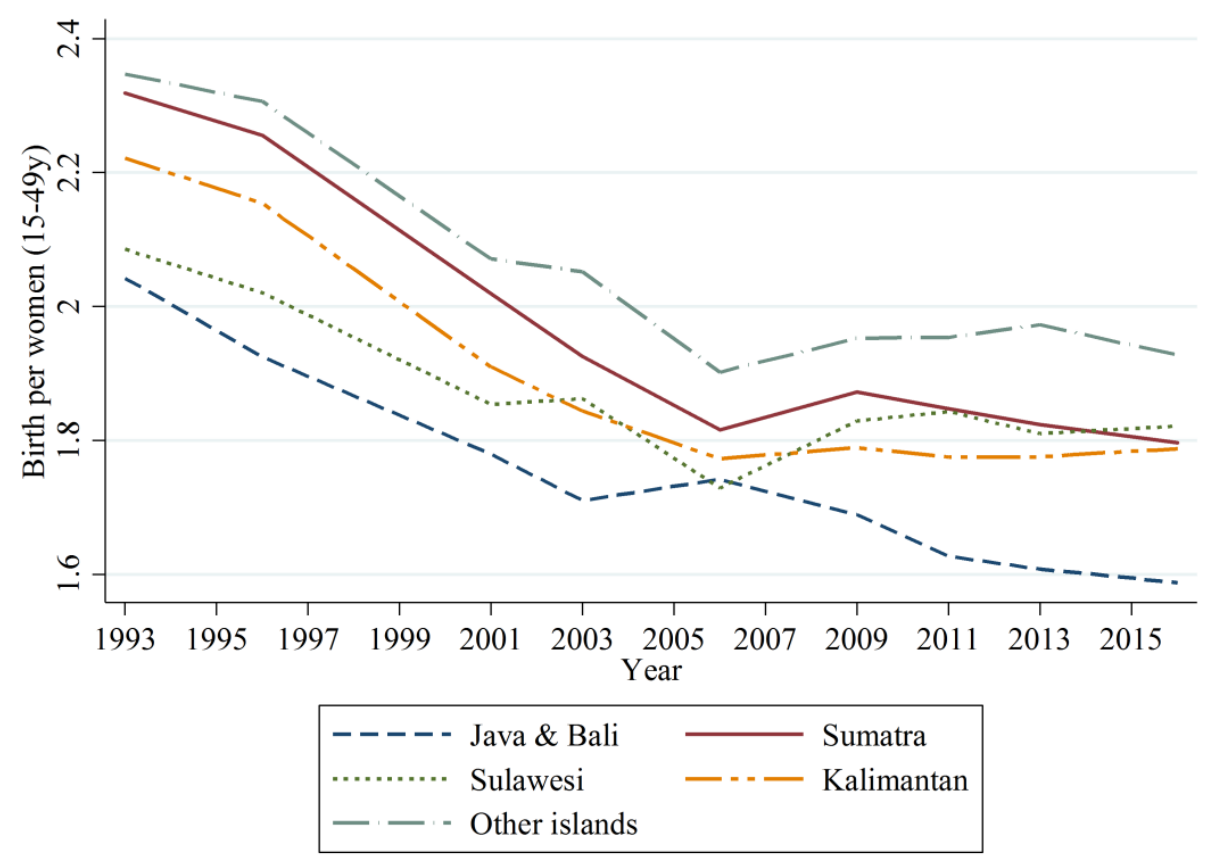

Source: SUSENAS data from 1993, 1996, 2001, 2003, 2006, 2009, 2011, 2013 and 2016.

We use the National Labor Force Survey (SAKERNAS) to capture labor market characteristics of working age individuals. The SAKERNAS provides information on labor supply, the sector of activity, as well as on wages of men and women in different sectors. The SAKERNAS has regency identifiers from 2000 onwards. The survey is, however, representative at the regency level only since 2007. We use the SAKERNAS to calculate province-level controls since 1996, and to test for the effects of oil palm on labor market outcomes at regency level in the time period 2001 to 2015.

Additional control variables are derived from the Demographic and Health Surveys (DHS), the Census, as well as from different administrative data sources. We use the DHS to control for child mortality, which is defined as the number of child deaths between the ages of one to five years per 1000 live births. Furthermore, we use the DHS to estimate the effect of the oil palm expansion on current fertility, in addition to the total number of live births per woman. Finally, the DHS provides a number of interesting outcomes that proxy for female bargaining power, such as the difference between actual and desired fertility, women's influence on household and personal decisions, and women's control over their own income. Since neither SAKERNAS nor SUSENAS provide detailed information on internal migration, we use data from the Indonesian Census to calculate the share of 
individuals who ever migrated as well as the share of individuals who migrated in last 5 years at the regency level. ${ }^{24}$ Administrative data, such as poverty lines, are retrieved from Badan Pusat Statistik (BPS) and public revenue data are retrieved from the Ministry of Finance, Information System for Sub-National Budget. We also calculate average altitude at regency level from the NASA Shuttle Radar Topographic Mission (SRTM) digital elevation data. ${ }^{25}$

Finally, we use data from the Global Agro-Ecological Zones (GAEZ) database for our instrumental variables approach. The GAEZ provides agro-climatically attainable yield data for different crops under specific levels of inputs and management conditions. It uses information on agro-climatic conditions to predict attainable yield based on agronomic models in grid cells of 5 arc-minute and 30 arc-second (approximately 10x 10km) resolution (Fischer et al. 2012). We use the maximum attainable yield of palm oil under rain-fed conditions and low-input management for the average climate in the baseline period 1961-1990. The low-input level was chosen since its predictions yield the highest correlation with the actual expansion of oil palm in a test area (Jambi Province, Sumatra), where land-use classification based on LANDSAT satellite imagery is available (Melati et al. 2014). Low-input levels may be not adequate by definition since fertilizer use is common in oil palm cultivation, however, the usage may not be optimal, especially in the case of smallholder farming. The GAEZ data is used to calculate the average attainable yield of oil palm within a regency by aggregating the pixel values within 1993 regency boundaries. Figure 8 illustrates our calculations.

\footnotetext{
${ }^{24}$ We use the subsample of the census from the IPUMS database.

${ }^{25}$ Country-specific data can be downloaded from http://www.diva-gis.org/gdata.
} 
Figure 8: Regency-wise attainable yield for oil palm in Indonesia

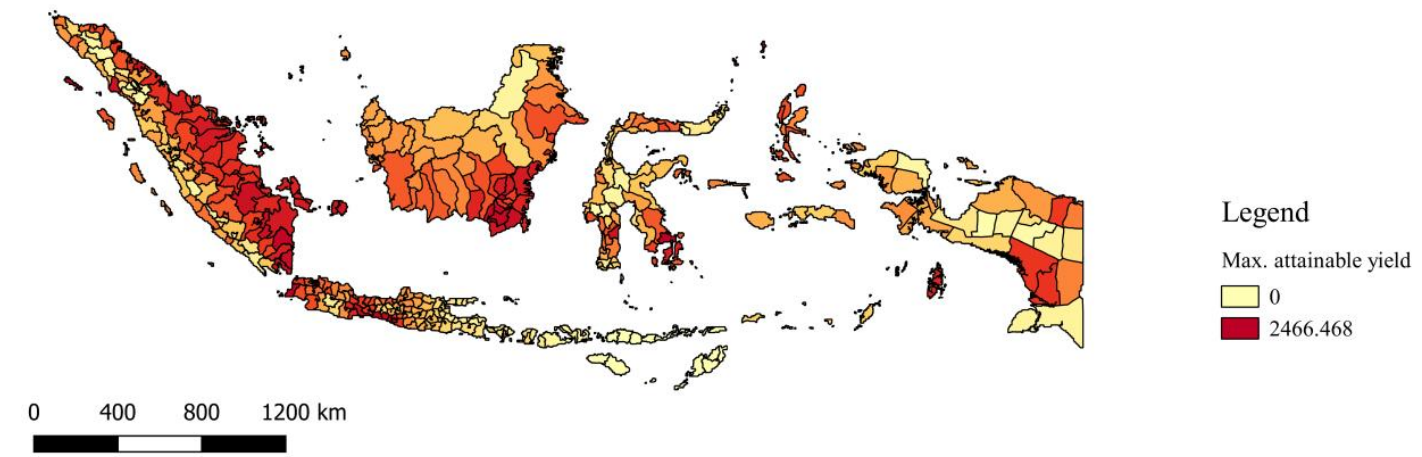

Source: GAEZ. Max attainable yield is in palm oil (kg/ha). Conversion factor to oil palm fresh fruit bunches is 0.225.

\subsection{Estimation strategy}

Eliciting a causal effect of oil palm expansion on fertility involves two major challenges: First, we cannot observe a lot of regency characteristics such as cultural and political traits, as well as time variant macro shocks such as the transmigrant program, which may correlate with the proliferation of oil palm as well as the fertility transition. ${ }^{26}$ Second, reverse causality such as high or low population growth which may induce oil palm expansion, could be driving our results.

We therefore use an instrumental variables approach combined with regency-fixed effects to identify causal effects. Our instrument combines time-invariant agro-climatic suitability for oil palm with the national expansion of oil palm, and is inspired by Duflo \& Pande (2007). We interact the suitability of oil palm at regency level with the annual expansion of oil palm at national level. We assume that national expansion is driven by world market prices and policies of the central government and is not affected by idiosyncratic regional developments which could be correlated with both fertility and oil palm expansion.

This approach provides a prediction of how much area in each regency should be cultivated with oil palm in a given year, based on the regency's suitability for oil palm cultivation, and the national expansion of oil palm area. This delivers an instrument which highly correlates with the actual expansion, since next to access to land and transport costs,

\footnotetext{
${ }^{26}$ The importance of cultural and linguistic boundaries for fertility patterns is shown inter alia by Munshi \& Myaux 2006; Cleland \& Wilson 1987).
} 
agro-ecological suitability is a major determinant of agricultural land-use patterns. Importantly, we expect this instrument to be exogenous, i.e. to affect fertility only through its effect on oil palm expansion, and not through any other mechanisms.

A couple of possible threats to identification remain. The first is that other crops have similar agro-ecological requirements as oil palm and have a similar expansion pattern over time. This would imply that our instrument captures very different levels of initial agricultural and probably also economic activity, which also suggests different trends in fertility outcomes. A second threat to identification could be that our instrument captures general geographic characteristics (such as altitude) which strongly correlate with initial levels of development and fertility. It seems fairly plausible that differences in initial levels in development and fertility also imply differential trends of fertility reduction. In order to address these concerns, we allow time trends between regencies to vary based on initial values of fertility, electrification, share of agricultural employment in total employment, and agricultural wages. ${ }^{27}$ Our results are not affected by the inclusion of these controls. A third caveat in our identification strategy could be the high regional concentration in the oil palm expansion. As depicted in Figure 6, the oil palm expansion started mainly in Sumatra and spread only later to Sulawesi and Kalimantan. The fact that these regions also had very different initial levels of fertility could indicate cultural and regional differences in underlying preferences towards children, and consequently very different trends in fertility declines without oil palm expansion. Also higher initial fertility could lead to a more rapid decline in subsequent years, when regions start to catch up with the development of the central regions, introducing a spurious negative correlation. It is thus not unlikely that these islands were on different fertility trends, or that regional shocks affected both the oil palm expansion as well as fertility. Therefore, we control for island-year fixed effects in all our estimations.

\footnotetext{
${ }^{27}$ We do not control for differential trends based on attainable yield, since the oil palm expansion is fairly linear through time and the regression would suffer from high collinearity. We also control for differential trends based on altitude as a robustness check and our results are robust to this control.
} 
Our first stage is then:

$$
O P_{j s t}=\alpha_{o}+\alpha_{1}\left(A Y_{j s} * O P A_{t}\right)+\alpha_{2} X_{i j s t}+\alpha_{3}\left(M_{j s} * y_{t}\right)+y_{t}+\gamma_{s t}+\mu_{j}+\omega_{i j s t},
$$

where $O P_{j s t}$ is the share of smallholder oil palm area in regency $j$, island $s$ and time $t$. $A Y_{j s}$ is the attainable yield of oil palm in regency $j$ and $O P A_{t}$ is the oil palm area in hectare at national level in year $t . X_{i j s t}$ is a vector of individual controls, such as a woman's age. $M_{j s}$ are initial characteristics, such as regency-average fertility, share of workers in agriculture, share of households with access to electricity, and province-average agricultural wages. These initial characteristics are multiplied with a time trend $y_{t}$. We also control for year fixed effects $y_{t}$, island-year fixed effects $\gamma_{s t}$ and regency fixed effects $\mu_{j}$.

The second stage of our fixed effects 2SLS models is:

$$
F R_{i j s t}=\beta_{o}+\beta_{1} \widehat{O P}_{j s t}+\beta_{2} X_{i j s t}+\beta_{3}\left(M_{j s} * y_{t}\right)+y_{t}+\gamma_{s t}+\mu_{j}+\varepsilon_{i j s t},
$$

where $F R_{i j s t}$ is the number of children born to each woman aged 15 to 49 in regency $j$, island $s$ and year $t$. All remaining variables are defined as in equation (9), and $\varepsilon_{i j s t}$ is an individual error term. We use household survey weights in all our estimations and cluster standard errors at regency level.

Despite having annual data on the oil palm expansion from administrative sources, we use a long-differences approach, and concentrate on the changes in oil palm cultivation and fertility over 10-year periods (1996, 2006 and 2016). This is because we expect the effect of oil palm on fertility to work through different mechanisms, which materialize over different time periods, and which might feed back into each other. By using 10-year differences we hope to account for the full impact on fertility. ${ }^{28}$

In order to test for the relative importance of different causal mechanisms, we employ a mediating analysis. That is, we first test if the oil palm expansion has a significant effect of the mediating variable of interest and then test if controlling for this variable in equation (10) affects the point estimate on the share of oil palm area.

\footnotetext{
${ }^{28}$ We test for the importance of time-lags as additional robustness check.
} 


\subsection{Results}

\subsubsection{Effect of oil palm expansion on fertility}

In Table 8 we present our main results for equation (10). In column (1) we use ordinary least squares (OLS) with regency and time fixed effects. Column (2) additionally includes island-year fixed effects and initial level of fertility, electrification, share of agricultural labor in total employment, and wages in agriculture multiplied with a time trend. In columns (3) to (6) we use the instrumental variables approach described in Section 4.5. To assess the robustness of our findings, we subsequently add different controls. We add island-year fixed effects in column (4). In column (5) we then additionally control for differential time trends based on initial levels of fertility, electrification, share of agricultural employment, and agricultural wages. In column (6) we add a woman's age to the controls. The age control is included because the age structure in a regency strongly determines fertility and it is not unlikely that age structure are on different fertility trends.

Table 8: Effect of oil palm expansion on fertility

\begin{tabular}{lcccccc} 
& & & \multicolumn{2}{c}{$\mathbf{1 9 9 6 - 2 0 0 6 - 2 0 1 6}$} & & \\
& $(1)$ & $(2)$ & $(3)$ & $(4)$ & $(5)$ & $(6)$ \\
& OLS & OLS & IV & IV & IV & IV \\
\hline Share of smallholder & -0.422 & -0.278 & $-6.421^{* *}$ & $-14.841^{* *}$ & $-4.614^{* *}$ & $-6.707^{* *}$ \\
OP area in regency & $(0.414)$ & $(0.376)$ & $(2.572)$ & $(7.167)$ & $(2.117)$ & $(2.727)$ \\
& & & & & \\
Regency \& year FE & Yes & Yes & Yes & Yes & Yes & Yes \\
Island-year FE & No & Yes & No & Yes & Yes & Yes \\
Initial levels * year & No & Yes & No & No & Yes & Yes \\
Woman's age & No & No & No & No & No & Yes \\
& & & & & 564.73 \\
F-stat & 377.129 & 413.431 & 218.526 & 81.247 & 289.315 & 10.586 \\
Kleibergen F-stat & & & 15.352 & 8.192 & 10.586 & 602758 \\
Observations & 602758 & 602758 & 602758 & 602758 & 602758 &
\end{tabular}

The results show a consistently negative effect of the oil palm expansion on fertility. The effect is always statistically significant in the instrumental variables estimations. Column (6) is the preferred specification, as it presents the most conservative estimates that allow for differential fertility shocks between islands and between regencies with different levels of initial fertility and economic development.

Between 1996 and 2016 the average share of oil palm area at regency level increased from $0.26 \%$ to $1.57 \%$. This would lead to a decrease of 0.088 children born per woman over a period of 20 years. Between 1996 and 2016 fertility decreased from 2.133 to 1.63 in our sample, the oil palm expansion could hence explain up to $17 \%$ of the observed fertility reduction. 
The estimated effect size is increasing significantly when moving from OLS to our instrumental variables approach. This could indicate a weak instrument problem; however, our first-stage Kleibergen-Paap rk Wald F-statistic suggests that our instrument is reasonably strong in most regressions ranging between 8.192 and 15.352 . There could be three other reasons for having lower OLS than IV estimates. First, our IV estimates capture the local average treatment effect of oil palm expansion. We thus show an effect for regencies where oil palm was planted because of favorable agro-ecological conditions and not for example because of policy regulations. Plantations in favorable agro-ecological conditions are likely to have higher returns, leading to higher income effects and potentially stronger fertility reductions. We caution hence that our estimates present an upper bound to the average treatment effect. Second, the IV approach might have corrected endogeneity biases. Oil palm plantations which were incentivized by government policies rather than agro-ecological suitability have been targeting regions with low population density and poor economic development in order to pursue the national development agenda, filling the demand for labor with migrants from densely populated islands (Gatto et al. 2017). Migrants from densely populated regions could have had lower fertility preferences, which could have reduced the initial level of fertility and possibly the subsequent decrease. This could explain an upward bias in OLS. Lastly, administrative data on the expansion of oil palm could involve significant measurement error, while the suitability index is based on agro-climatic, soil and terrain conditions which may be more precisely measured. Thus, our IV approach may correct measurement errors, which induce an attenuation bias in our OLS estimates.

We do not expect effects to be uniform across the population. Figure 9 reports the result disaggregated by age groups based on the specification in column (6) in Table 8 . The figure shows that the negative effect is only statistically significant in the age groups between 25 and 39, peaking for the age-group 30 to 34 . The apparent zero effect for younger age groups is likely to be driven by our long-difference estimation strategy, combined with a relatively static fertility measure. The 25 to 29 age cohort, for which we observe a significant effect, was only 15 to 19 years old 10 years earlier. The negative fertility effect of oil palm could have hence taken place anywhere between the age of 15 to 29. The insignificant effect for older women is in line with our expectations. 
Figure 9: Effect of oil palm on fertility by age cohorts

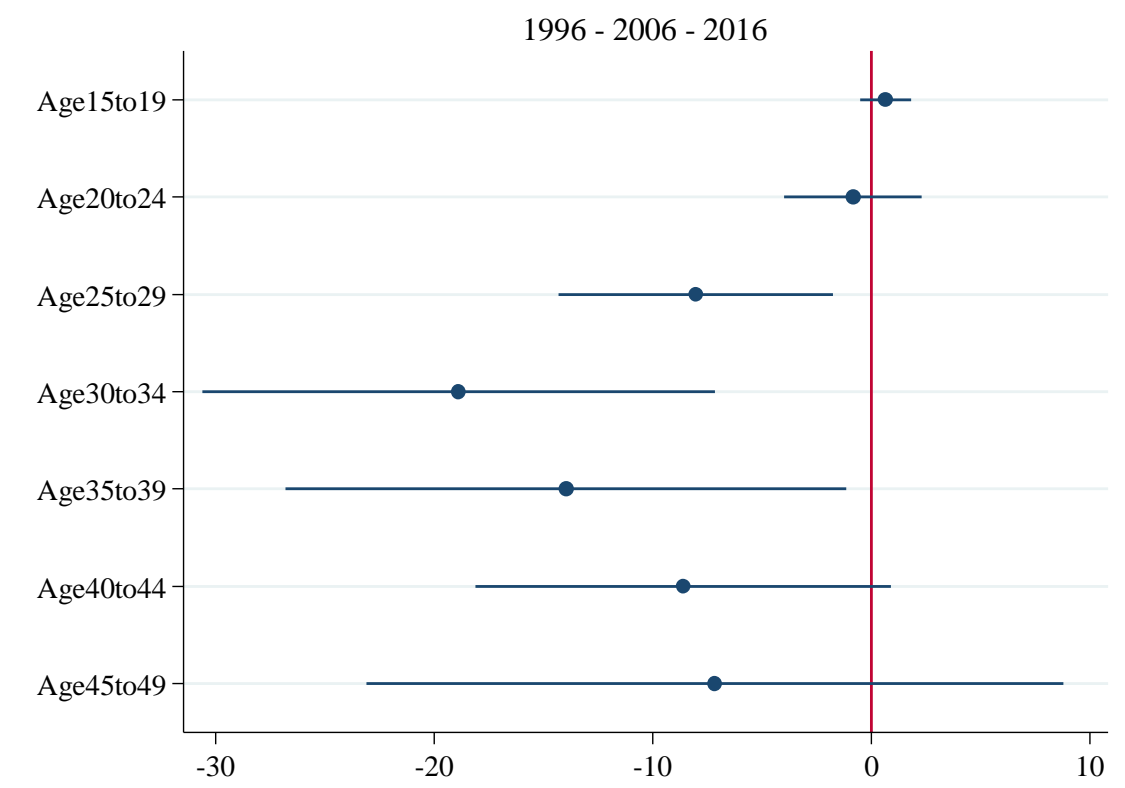

Notes: Marginal effects and 90\% CI are reported. Standard errors are clustered at regency level. IV estimates are reported. All regressions control for national oil palm area, woman's age, regency and year fixed effects, island-year fixed effects, and initial values of fertility, electrification, share of agriculture in total employment and agricultural wages times year.

\subsubsection{Robustness checks}

We conduct several additional checks to gauge the robustness of our findings. First of all, we use the PODES dataset to measure oil palm expansion at the village level. Unfortunately this information is only available in 1993 and 2003. We replicate the specifications used in Table 8 with the new variable on oil palm expansion in Table A13 in the Appendix. The results show a consistent negative and significant effect of oil palm expansion on fertility, confirming our previous results.

In the next step, we test in the 1996 to 2016 sample if our results are sensitive to our choice of specification. Results are reported in the Appendix, Table A14. First, we run the same regression without survey weights. The change in effect size is negligible, but the Kleibergen F-Statistic increases. Second, we include province-time trends instead of island-year fixed effects. Again, our results remain robust and of the same magnitude, although the first stage F-Statistic decreases. Third, we use attainable yield of oil palm under rain-fed conditions and medium-input management instead of low-input management as cross-sectional component of our instrumental variable. Again, the results are not affected. Fourth, we test if our results are sensitive to excluding Java, Indonesia's 
main island, from our sample. Close to $40 \%$ of Indonesia's population lives on Java. At the same time, Java grows oil palm only to a very small extent. We find in column (4) that the effect remains highly significant and the precision of the first stage increases, since the low levels of oil palm cultivation in Java are not driven by low suitability but little available land. Fifth, we control for each regency's average altitude multiplied by a time trend. As discussed previously, one concern with the validity of our instrument stems from the fact that the attainable yield of oil palm changes with altitude. Since regencies with high altitude are generally more remote, they might also be on different trends of economic development and fertility compared to low-land regencies. If this effect is not picked up by any of our trends, this could possibly bias our results. As can be seen in column (5), the effect size remains stable and significant. Only the Kleibergen F-stat drops to 7.7, which is to be expected given that we substantially reduce the variation in our instrument. Finally, in columns (6) and (7), we compare the effect of oil palm on fertility between producer types. We use the sample of 2005-2011-2016 since we do not have consistent data for total oil palm area before 2005 and no data in 2006 . We find that the effect on fertility is lower when using total oil palm area rather than smallholder oil palm area as explanatory variable, but the effect remains statistically significant. One possible explanation could be that the income effect is more pronounced for smallholders compared to agricultural wage laborers. We also show in Table A15 that our results are robust to using 5-year differences rather than 10-year differences.

One weakness of our measure of fertility is that it captures the total number of children born per woman, rather than current fertility. This variable is sensitive to past changes in fertility and, given serial correlation in the expansion of oil palm, might capture changes in fertility that happened some time ago. Moreover, the full effect of oil palm expansion on income might only be realized after some years since oil palm trees are only productive two to three years after being planted. We therefore split our sample by age groups and test if lagging the oil palm expansion by two or five years affects the observed pattern by age group. Results are reported in the Appendix, Figure A3. We find that the effect of oil palm on fertility is now more pronounced for the older cohorts. This is expected since the lagged oil palm expansion is restricted to fertility decisions which occurred at least two or five years ago, limiting the effect on young cohorts. 
As additional robustness check, we use fertility data from the DHS to illustrate differences between our fertility variable and current fertility. The DHS data is only representative at the province level, which is why our identification strategy has to rely on controlling for province and time fixed effects. We use DHS data from the years 1997, 2002, 2007 and 2012. Figure A4 in the Appendix reports the results. The figure on the left shows the effect on total fertility (consistent with our fertility measure in SUSENAS), while the figure on the right shows the effect on current fertility. Current fertility is defined as the number of births for each woman in the last 12 months, and is the standard measure used to construct the TFR. The figure on the left overlaps 1:1 with our results from SUSENAS, which shows that our results are robust to the use of alternative data and identification strategies. In the figure on the right, we can see that current fertility reductions are driven by two age groups: First, the 20-24 year olds, and, second, women aged 35-44. Younger women may be reducing current fertility because they delay marriage and stay longer in school. Consistently, the observed positive effect on fertility in the 25-29 age group might be a catch-up effect. However, the fact that 35-44 year old women also reduce their current fertility could indicate changing preferences towards very large families. While the DHS data confirms our results, it also indicates that our fertility variable limits the scope for detailed cohort analyses.

\subsubsection{Transmission mechanisms}

\subsubsection{Income}

In Section 4.2, we cite evidence that the oil palm expansion induced positive income effects. Increasing income would lead to decreasing fertility rates in Becker's Q-Q model. We test this proposition using consumption expenditure per capita as proxy for income, calculated at the household level in the SUSENAS data. Table 9 presents the results. In column (1), we show that oil palm expansion has a significantly positive effect on consumption expenditures. Columns (2) and (3) show that this effect is driven by households whose head is involved in agriculture as his main job. In the subsequent columns, we test if the effect of oil palm expansion on fertility declines when controlling for changes in per capita consumption expenditure. We find in column (4) that the point estimate on oil palm decreases from -6.7 to -4.9 after controlling for household-level 
consumption expenditure per capita. In columns (4) and (5), we split the sample by the primary occupation of the household head. While the income effect seems to be driven by households who are engaged primarily in agriculture, fertility effects can be observed in both groups. Taken together these results suggest that part of the effect on fertility can be explained by an income effect, but that other mechanisms must be at work, too.

Table 9: Transmission mechanisms - Income effect

\begin{tabular}{|c|c|c|c|c|c|c|}
\hline & \multicolumn{6}{|c|}{ 1996-2006-2016 } \\
\hline & (1) & (2) & (3) & (4) & (5) & (6) \\
\hline & Consumption & Consumption & Consumption & Fertility & Fertility & Fertility \\
\hline & Exp.p.c. & $\begin{array}{l}\text { Exp. p. c. } \\
\text { HH in agr. }\end{array}$ & $\begin{array}{l}\text { Exp. p. c. } \\
\text { HH in non- } \\
\text { agr. }\end{array}$ & & HH in agr. & $\begin{array}{l}\mathrm{HH} \text { in non- } \\
\text { agr. }\end{array}$ \\
\hline Share of smallholder & $3.376^{*}$ & $3.822^{* * *}$ & 1.611 & $-4.887^{*}$ & $-6.222^{* * *}$ & $-8.488^{* * *}$ \\
\hline $\mathrm{OP}$ area in regency & $(2.046)$ & (1.902) & $(2.537)$ & (2.797) & $(2.627)$ & $(3.800)$ \\
\hline Consumption & & & & $-0.513^{* * * *}$ & & \\
\hline expenditures p.c. & & & & $(0.016)$ & & \\
\hline F-stat & 161.573 & 172.506 & 170.022 & 630.011 & 365.880 & 551.342 \\
\hline Kleibergen F-stat & 10.706 & 12.995 & 8.481 & 10.577 & 13.209 & 8.276 \\
\hline Observations & 2294491 & 1102206 & 1192285 & 602758 & 280546 & 322212 \\
\hline
\end{tabular}

\subsubsection{Child labor}

As discussed in Section 4.3, decreasing returns to child labor could increase the price of child quantity. We therefore test if the oil palm expansion is associated with changes in child labor, and if average child labor at the regency level mediates the effect of oil palm on fertility.

Child labor can be calculated only for children between 10 and 14 years. ${ }^{29}$ Results are reported in the Appendix, Table A16. In summary, we find no evidence that child labor explains the negative effect of oil palm on fertility. Oil palm does not seem to affect total child labor, nor on-farm or gender-specific child labor. Likewise, controlling for child labor or on-farm child labor does not change the effect of oil palm on fertility. Since schooling and child labor are substitutes in time use, the finding that oil palm does not affect child enrollment in Table A17 gives additional support to our findings. We conclude

\footnotetext{
${ }^{29}$ We do not have any data on working activities of children below the age of ten.
} 
that changes in child labor are not an important mechanism in explaining the negative effect of oil palm expansion on fertility.

\subsubsection{Maternal opportunity costs of time}

We argue in our theoretical model that maternal opportunity costs of time might decrease or increase depending on whether the oil palm expansion triggers local economic development and how this affects wages. In the absence of such effects, oil palm may only induce labor savings, which would reduce the opportunity costs of child rearing. We therefore start by estimating the effect of oil palm on wages and labor supply. Columns (1) and (2) of Table 10 show a positive and significant effect of oil palm on wages in the nonagricultural sector and a negative (albeit not statistically significant) effect of oil palm on women's wages in the agricultural sector in the SAKERNAS dataset from 2001 to $2015 .^{30}$

In Table A18 in the Appendix, we use a set of different control variables to identify the drivers of the increase in non-agricultural wages for women. We find that changes in average consumption expenditures at the regency level as well as government revenues from own sources explain the positive wage effect of the oil palm expansion. In contrast, changes in women's educational attainment or in the sectoral composition of the workforce do not seem to explain the increase in wages. This suggests that local economy effects driven by income growth are primarily responsible for increasing women's nonagricultural wages.

\footnotetext{
${ }^{30}$ The reason for restricting the sample to 2001 is that older SAKERNAS rounds do not contain regency identifiers and the questionnaire is only consistently eliciting all variables starting from 2001.
} 
Table 10: Transmission mechanisms - Opportunity costs of time

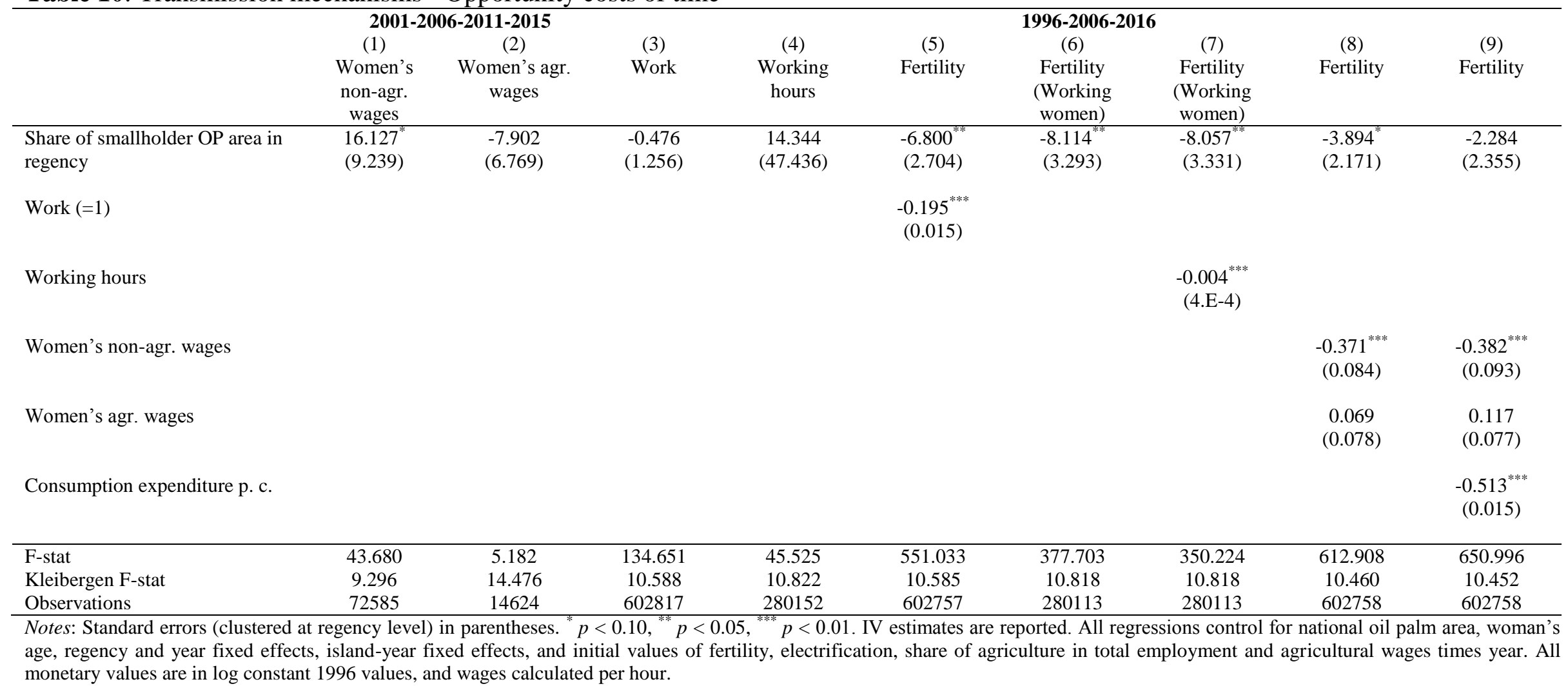


We proceed by testing if increased wages led to higher labor supply. In columns (3) and (4) of Table 10 we show that women do not change their labor supply either at the extensive margin or at the intensive margin. Little surprisingly, controlling for labor supply does also not change the effect of oil palm on fertility, as reported in columns (5) to (7). Interestingly, however, increases in women's wages seem to mediate strongly the effect of oil palm on fertility (columns 8 and 9). And as can be seen by comparing column (9) to Table 9, col. (4), this cannot be explained by an income effect. ${ }^{31}$

In order to analyze how wage increases affect fertility if not via changes in labor supply, we proceed by testing if oil palm affects the sector in which women work (Table 11, cols. 1 and 2). Consistent with the finding of rising non-agricultural wages, we find that women shift out of agriculture and into the services sector. However, we do not find that controlling for the sector in which a woman works changes the coefficient of oil palm on fertility substantially. Controlling for whether a woman works in agriculture reduces the coefficient on oil palm for working women from -8.1 (Table 10, col. 6) to -7.5 (Table 11, col. 5). ${ }^{32}$ Sectoral shifts alone are thus not able to explain the strong mediating effect of women's wages.

Table 11: Transmission mechanisms - Opportunity costs of time II

\begin{tabular}{|c|c|c|c|c|c|c|}
\hline & & & 1996- & $6-2016$ & & \\
\hline & (1) & (2) & (3) & (4) & (5) & (6) \\
\hline & $\begin{array}{l}\text { Work in } \\
\text { agriculture }\end{array}$ & $\begin{array}{l}\text { Work in } \\
\text { service } \\
\text { sector }\end{array}$ & $\begin{array}{l}\text { Education } \\
\text { women } \\
(15-25 y)\end{array}$ & $\begin{array}{l}\text { Education } \\
\text { women } \\
\text { (26-35y) }\end{array}$ & $\begin{array}{l}\text { Fertility } \\
\text { (Working } \\
\text { women) }\end{array}$ & Fertility \\
\hline Share of smallholder & $-2.793^{* * *}$ & $2.600^{* * *}$ & $5.235^{*}$ & $10.036^{* * 6}$ & $-7.514^{* * *}$ & $-5.601^{k *}$ \\
\hline OP area in regency & (1.307) & (1.160) & $(2.820)$ & $(4.957)$ & (3.192) & $(2.736)$ \\
\hline $\begin{array}{l}\text { Work in agriculture } \\
(=1)\end{array}$ & & & & & $\begin{array}{l}0.215^{* * *} \\
(0.019)\end{array}$ & \\
\hline $\begin{array}{l}\text { Women's education } \\
\text { level }\end{array}$ & No & No & No & No & No & Yes \\
\hline F-stat & 51.713 & 52.839 & 357.177 & 262.315 & 382.410 & 719.396 \\
\hline Kleibergen F-stat & 10.822 & 10.822 & 10.349 & 10.614 & 10.812 & 10.578 \\
\hline Observations & 280152 & 280152 & 211238 & 181646 & 280113 & 602758 \\
\hline $\begin{array}{l}\text { Notes: Standard error } \\
\text { reported. All regressi } \\
\text { fixed effects, and initi } \\
\text { times year. Education } \\
\text { secondary and tertiary }\end{array}$ & $\begin{array}{l}\text { ustered at } \\
\text { control fo } \\
\text { alues of fe } \\
\text { vel is a } \\
\text { ooling. }\end{array}$ & $\begin{array}{l}\text { y level) } \\
\text { nal oil } \\
\text { electrific } \\
\text { ical var }\end{array}$ & $\begin{array}{l}\text { ntheses. } \\
\text { ea, woman } \\
\text { hare of agr } \\
\text { Categories }\end{array}$ & $\begin{array}{l}10,{ }^{* *} p< \\
\text { e, regency } \\
\text { re in total } \\
o \text { degree, }\end{array}$ & $\begin{array}{l}\text { 雬** } p<0 . \\
\text { ear fixed } \\
\text { yment an } \\
\text { ary, junio }\end{array}$ & $\begin{array}{l}\text { estimates } \\
\text { s, island- } \\
\text { ultural we } \\
\text { ndary, se }\end{array}$ \\
\hline
\end{tabular}

31 The SAKERNAS 1996 does not provide regency identifiers. We thus control for average provincelevel wages in columns (8) and (9). We merge SAKERNAS data from 2015 with SUSENAS data from 2016.

${ }^{32}$ The effect of oil palm on labor supply and sector of work is robust to using SAKERNAS instead of SUSENAS data, see Table A19. 
To understand what else could drive the relationship between increasing wages and fertility reductions, we regress wages at different educational levels on oil palm in Table 12. Again, we use wage data from SAKERNAS. We find that the oil palm expansion mostly increased wages for more highly educated individuals, in particular for men and women with tertiary education, as shown in column (3) and (6). ${ }^{33}$

Table 12: Effect of oil palm on wages - Returns to education

\begin{tabular}{|c|c|c|c|c|c|c|}
\hline & \multicolumn{6}{|c|}{ 2001-2006-2011-2015 } \\
\hline & (1) & (2) & (3) & (4) & (5) & (6) \\
\hline & \multicolumn{3}{|c|}{ Women's wages } & \multicolumn{3}{|c|}{ Men's wages } \\
\hline & $<$ Primary & Secondary & Tertiary & $<$ Primary & Secondary & Tertiary \\
\hline Share of smallholder & -0.476 & 1.508 & $38.828^{* * * *}$ & -10.141 & $13.110^{*}$ & $31.250^{* * *}$ \\
\hline OP area in regency & $(8.761)$ & $(8.415)$ & $(14.035)$ & $(6.641)$ & $(7.570)$ & $(14.574)$ \\
\hline F-stat & 13.300 & 57.607 & 72.715 & 37.428 & 272.367 & 67.720 \\
\hline Kleibergen F-stat & 8.877 & 9.568 & 12.212 & 9.335 & 9.851 & 9.817 \\
\hline Observations & 31653 & 37951 & 17605 & 73130 & 97042 & 17038 \\
\hline
\end{tabular}

Rising returns to education might have induced a reallocation of time away from child rearing to education. In Table 11 columns (3) and (4), we show that the educational attainment of women in the age groups 15-25 and 26-35 increased significantly due to the oil palm expansion. The latter group is included because we work with 10-year differences and therefore this group is also likely to be affected by changes in oil palm expansion. In column (6), we add controls for the educational attainment of a woman to the regression of fertility on oil palm. This decreases the point estimate of oil palm on fertility from -6.7 to -5.6 .

Taken together, these results indicate that oil palm triggered local economic development, which raised average wages as well as the returns to education. This increased the opportunity costs of child rearing, and women opted to change into the

\footnotetext{
${ }^{33}$ These results do not necessarily imply that there are no income effects for low education households. Although wages are reported individually, wages obtained in family work are not reported and most likely included in the wage reported by the household head. Since women left family work (see Table A19), this might have decreased the contribution of family labor to own farm work and sharecropping and therewith the wage men reported. Multiple jobs are also more likely in the low education sector. By only measuring the wage from the main job, we might also miss the income effects stemming from reallocating working time between jobs. Still, also comparing wage increases between the secondary and tertiary education group, where the aforementioned issues play a less significant role, indicates that returns to education increased.
} 
service sector and to invest more time in their education, both of which then led to reductions in fertility.

\subsubsection{Returns to children's education}

Changes in returns to education also affect the price of child quality, encouraging parents to invest more in the education of their children and to reduce fertility. In Table 13, columns (1) and (2), we regress the educational attainment of boys and girls between the age of 12 and 14 on oil palm. ${ }^{34}$ We use the age of 12 as cutoff point since children are unlikely to have finished primary school before the age of 12 . We observe a positive effect, which, however, is only significant for girls in column (2). One reason could be that since the educational attainment of girls is in general lower investing in their education provides higher returns. Although the increase is only small, literature argues that even small shifts in child quality can induce major reductions in child quantity. In column (3), we test if investing in the quality of children explains part of the effect of oil palm on fertility by including the average educational attainment of girls and boys between the age of 12 and 14 in a regency as controls. We restrict the variable to below 14 years in order not to confound the effect on an increase in women's education with that of children. We find that the oil palm effect is decreasing from -6.7 to -5.4 . We find similar results when expanding the sample to children aged 12-19 (as reported in Table A21 in the Appendix). In column (4), we add consumption expenditures to control for the income effect, and all controls which are related with increasing maternal opportunity costs of time such as labor force participation, working hours, sector dummies and women's educational attainment. We additionally add women's wages in column (5) and find that this only reduces the coefficient oil palm from -3.8 to -2.9 , which implies that the major part of the wage effect found in Table 10 column (8) can be explained by an income effect, by changes in returns to education and to limited extent by sectoral shifts.

\footnotetext{
${ }^{34}$ We also estimate the effect of oil palm on enrollment rates in Table A17 in Appendix, and find no significant effects. The reason could be that enrollment does not necessarily imply effectively attending school or investing time and effort into achieving a higher degree.
} 
Table 13: Transmission mechanisms - Returns to children's education

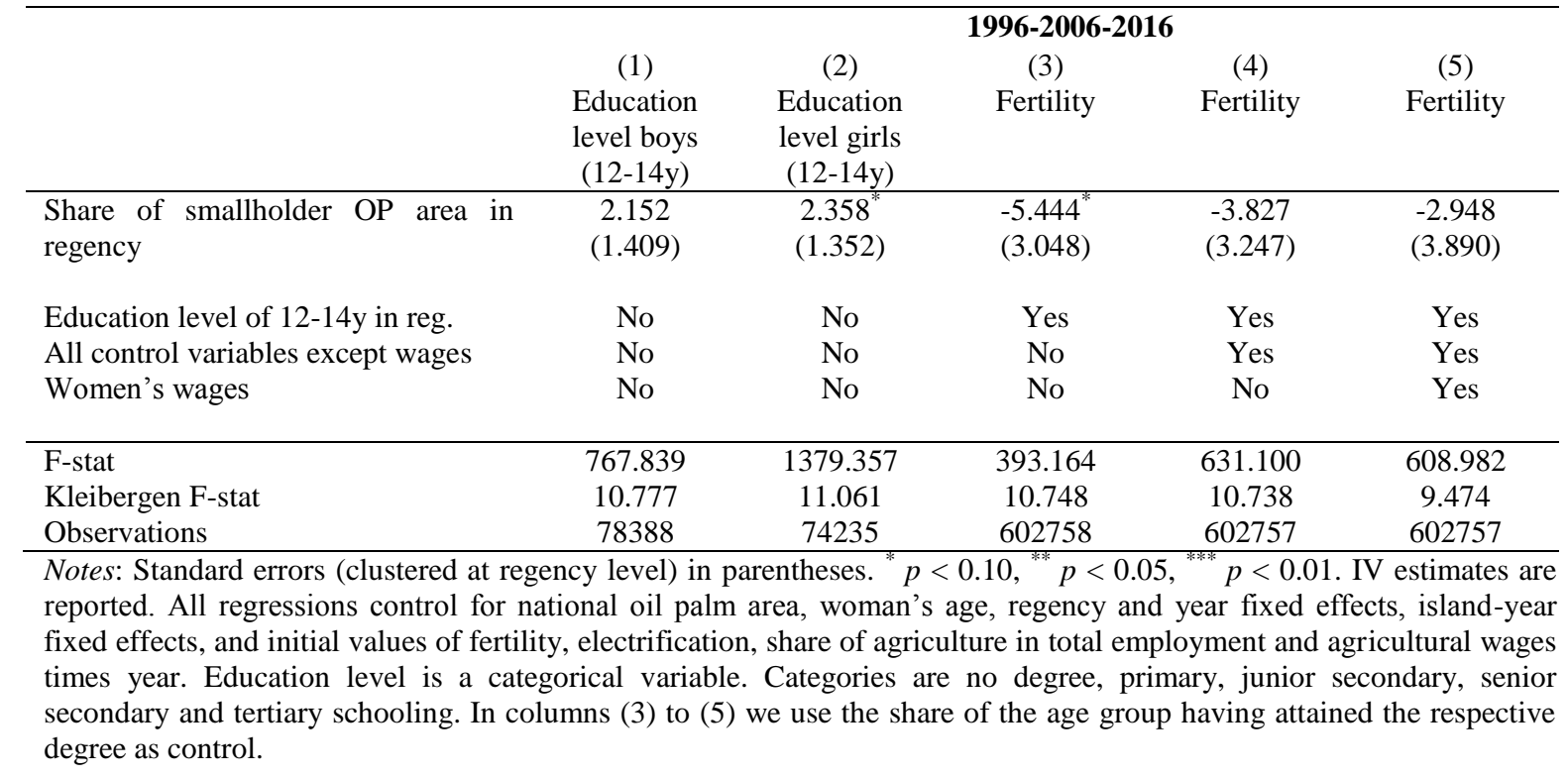

\subsubsection{Infrastructure}

The infrastructure effect suggests that the oil palm expansion might reduce the cost of child quality through better infrastructure. We use a wide range of variables as controls for infrastructure such as share of households with access to electricity from the public grid, share of villages with kindergarten, primary school, junior high school, asphalt main road and hospital. Table 14 reports the results in columns (1)-(6). We find a negative effect of oil palm on the share of villages with an asphalt road as main road, which is contrary to our expectations. However, initial oil palm expansion might have involved rather low quality roads and roads might have been asphalted only later with increasing income generation from oil palm. When using a three-year lag of the oil palm expansion, the effect is not statistically significant. ${ }^{35}$ Of all infrastructure indicators tested, we only find a positive and significant effect of oil palm for the share of villages with a hospital. We then proceed to testing if controlling for these infrastructure variables mediates the effect of oil palm on fertility in columns (7) to (10). ${ }^{36} \mathrm{We}$ do not find any evidence that reductions in the price of child quality - via infrastructure development - explain part of the observed negative effect of oil palm on fertility.

\footnotetext{
${ }^{35}$ Results are available from authors on request.

${ }^{36}$ We merge PODES 2014 on SUENAS 2016.
} 
Table 14: Transmission mechanisms - Infrastructure

\begin{tabular}{|c|c|c|c|c|c|c|c|c|c|c|}
\hline & \multicolumn{6}{|c|}{ 1996-2006-2014 } & \multicolumn{4}{|c|}{ 1996-2006-2016 } \\
\hline & (1) & (2) & (3) & (4) & (5) & (6) & (7) & (8) & (9) & $(10)$ \\
\hline & $\underset{\mathrm{n}}{\text { Kindergarte }}$ & $\begin{array}{l}\text { Primary } \\
\text { school }\end{array}$ & $\begin{array}{l}\text { Junior high } \\
\text { school }\end{array}$ & Asphalt road & Hospital & Electricity & Fertility & Fertility & Fertility & Fertility \\
\hline $\begin{array}{l}\text { Share of smallholder OP } \\
\text { area in regency }\end{array}$ & $\begin{array}{l}-0.468 \\
(1.988)\end{array}$ & $\begin{array}{c}0.327 \\
(0.274)\end{array}$ & $\begin{array}{l}-2.418 \\
(1.509)\end{array}$ & $\begin{array}{l}-2.380^{*} \\
(1.366)\end{array}$ & $\begin{array}{l}0.879^{* *} \\
(0.438)\end{array}$ & $\begin{array}{c}0.004 \\
(1.327)\end{array}$ & $\begin{array}{l}-7.725^{* *} \\
(3.004)\end{array}$ & $\begin{array}{c}-7.710^{* * * *} \\
(2.852)\end{array}$ & $\begin{array}{c}-7.707^{* * *} \\
(2.905)\end{array}$ & $\begin{array}{l}-8.450^{* *} \\
(3.440)\end{array}$ \\
\hline $\begin{array}{l}\text { Share of villages with } \\
\text { kindergarten in regency }\end{array}$ & & & & & & & $\begin{array}{c}0.112 \\
(0.127)\end{array}$ & $\begin{array}{c}0.104 \\
(0.129)\end{array}$ & $\begin{array}{c}0.104 \\
(0.130)\end{array}$ & $\begin{array}{c}0.113 \\
(0.136)\end{array}$ \\
\hline $\begin{array}{l}\text { Share of villages with } \\
\text { primary school in regency }\end{array}$ & & & & & & & $\begin{array}{c}0.908 \\
(0.813)\end{array}$ & $\begin{array}{c}1.032 \\
(0.802)\end{array}$ & $\begin{array}{c}1.031 \\
(0.794)\end{array}$ & $\begin{array}{c}1.110 \\
(0.865)\end{array}$ \\
\hline $\begin{array}{l}\text { Share of villages with junior } \\
\text { high school in regency }\end{array}$ & & & & & & & $\begin{array}{l}-0.223 \\
(0.166)\end{array}$ & $\begin{array}{l}-0.214 \\
(0.166)\end{array}$ & $\begin{array}{l}-0.214 \\
(0.167)\end{array}$ & $\begin{array}{l}-0.234 \\
(0.182)\end{array}$ \\
\hline $\begin{array}{l}\text { Share of households with } \\
\text { electricity in regency }\end{array}$ & & & & & & & & $\begin{array}{l}0.201^{* *} \\
(0.083)\end{array}$ & $\begin{array}{l}0.200^{* *} \\
(0.084)\end{array}$ & $\begin{array}{l}0.236^{* *} \\
(0.097)\end{array}$ \\
\hline $\begin{array}{l}\text { Share of villages with } \\
\text { asphalt road in regency }\end{array}$ & & & & & & & & & $\begin{array}{c}0.001 \\
(0.128)\end{array}$ & $\begin{array}{c}0.004 \\
(0.131)\end{array}$ \\
\hline $\begin{array}{l}\text { Share of villages with } \\
\text { hospital in regency }\end{array}$ & & & & & & & & & & $\begin{array}{c}0.301 \\
(0.423)\end{array}$ \\
\hline F-stat & 93.850 & 15.264 & 44.833 & 12.460 & 5.632 & 474.933 & 458.137 & 487.045 & 470.665 & 439.641 \\
\hline Kleibergen F-stat & 16.485 & 16.485 & 16.485 & 16.485 & 16.485 & 16.485 & 11.638 & 11.513 & 11.257 & 8.945 \\
\hline Observations & 620 & 620 & 620 & 620 & 620 & 620 & 601025 & 601025 & 601025 & 601025 \\
\hline
\end{tabular}




\subsubsection{Alternative explanations}

As mentioned in Section 3, other mechanisms that are not captured by the Q-Q model could explain a negative effect of oil palm on fertility, namely migration, changes in child mortality or female empowerment. These mechanisms are addressed in the following.

Columns (1) and (2) in Table A22 (in the Appendix) report the effect of oil palm on migration. We find that oil palm increases short-term migration (i.e., in the last five years), but not long-term migration. The data on migration is derived from the Indonesian Census and only available for 1995, 2000, 2005 and 2010. We thus merge the migration data with a one year lead on the SUSENAS dataset. ${ }^{37}$ Columns (3) to (5) assess the role of migration on fertility. We do not observe a significant change in the magnitude of the oil palm coefficient after controlling for either of the two migration variables. This suggests that migration does not explain the negative effect of the oil palm expansion on fertility.

We also control for changes in child mortality at province level. Unfortunately, the DHS data does not match well with our fertility data. We match DHS data form 1997, 2006 and 2012 with SUSENAS data from 1996, 2006 and 2011. Results are reported in the Appendix, Table A23. While we find that the expansion of oil palm is associated with decreasing child mortality, controlling for this variable again does not seem to affect the observed effect of oil palm on fertility. We therefore conclude that reductions in child mortality were no major transmission mechanism.

Finally, we explore the link between the oil palm expansion and female empowerment. Since we only have a few proxies for female empowerment in SUSENAS, we also use the DHS data to test if our results could be driven by an empowerment effect. One indication for female empowerment could be an increase in investments in children's education, in particular for girls, assuming that women have a higher preference for investing in their children (and in their daughters relative to their sons) than men. However, while we find that educational investments in children increase, the magnitude of the effect is almost identical for boys and girls, and could be explained equally well by the changes in returns to education (cf. Table 13). Therefore, we also test if the share of food expenditures in total household expenditures is increasing due to the oil palm expansion, assuming that women have a higher preference to spend money on food than men. Results are reported in Table

\footnotetext{
${ }^{37}$ Census data is missing for some regencies.
} 
A24 in the Appendix. We do not find any significant effect. Moreover, we test using DHS data if the oil palm expansion has an effect on several proxies of female empowerment such as the gap between desired and actual fertility, an index of female autonomy, and if women have control over their labor income. The results in Table A24 do not show any evidence that the oil palm expansion increased female empowerment. We also do not find any evidence that controlling for these variables changes the observed effect of oil palm on fertility (cf. Table A25).

We conclude that none of these mechanisms provide an alternative explanation for the negative effect of oil palm on fertility, and that the income effect as well as increasing wages and returns to education have a greater potential in explaining the negative effect of oil palm on fertility.

\subsection{Conclusion}

We contribute to the literature by disentangling the effect of a labor saving technology on fertility. Using the oil palm expansion in Indonesia as empirical example, we show that a labor saving technology does not necessarily lead to higher fertility. Rather positive income effects coupled with broader local economic development eventually decreased fertility in the context of oil palm production in Indonesia.

This essay presents evidence that oil palm induced labor savings under the condition of land scarcity, but also income gains in particular in the smallholder sector. Based on these observations, we develop testable hypotheses using Becker's Q-Q framework. Using an instrumental variables approach with regency-fixed effects, we find that the oil palm expansion significantly reduced fertility. This effect is persistent even after controlling for island specific time fixed effects and for differential trends depending on initial values of fertility, electrification, agricultural wages and sectoral shares. While our estimates likely represent an upper bound to the average treatment effect, they suggest that the oil palm expansion explains up to $17 \%$ of the fertility reduction observed in rural Indonesia in the time period between 1996 and 2016.

We then explore different transmission mechanisms and find evidence that the negative effect of oil palm on fertility is largely driven by income effects, as well as by local economy effects, which led to increasing wages in the non-agricultural sector as well as to 
higher returns to education. Our results suggest that increasing returns to education increased maternal opportunity costs of time resulting in a reallocation of time from child rearing to education. In addition, increasing returns to education induced higher investments in children's education, leading to further reductions in fertility. We conclude that the income growth coupled with local economic development outweighed the potential fertility increasing effects of the oil palm expansion.

While we find a negative effect of a labor saving technology on fertility, we argue this might depend on several preconditions. Indonesia has a long tradition of cash crop cultivation. People obtain a considerable part of food and non-food consumption goods from markets and are surrounded by a relatively well established institutional and infrastructural framework. In settings with less amenable conditions for the development of a prospering non-agricultural sector, labor force participation as well as wages could decrease, potentially decreasing maternal opportunity costs of time. Second, the availability of schools might matter. Our results suggest that investments in education were an important transmission mechanism. If high transaction costs impede such investments, fertility reduction might be less evident. Lastly, it is important to note that oil palm is largely adopted by smallholder farmers. Since oil palm adoption was voluntary, we have good reasons to expect that it was adopted due to expected income gains. A technology that is labor saving but not increasing the incomes of the majority of the population might have different effects. For example, mechanization in large-scale agriculture might increase labor productivity; however, an abundant labor supply might still depress wages, or restrict income gains to a minority. The income and local economic development effects might therefore be less pronounced or absent, which would substantially reduce the scope for fertility reduction. While our results indicate that a labor saving technology in the smallholder sector might be beneficial to fertility reduction, labor savings technologies in other sectors where income effects are smaller might not lead to the same reduction in fertility rates.

A number of caveats apply. Due to data limitations, the variable we use for fertility represents the number of all ever occurred live births per woman. Our variable thus captures events which possibly happened decades ago. While our results are robust to using lags and five-year differences, the potential serial correlation in oil palm expansion combined with our fertility variable limits the scope for a more detailed cohort analysis. 
Second, our data includes only a limited number of good indicators for child quality, as we can only observe variables related to schooling. And finally, we want to emphasize that although we find positive effects of oil palm expansion on consumption expenditures and on educational attainment, this does not imply that oil palm is to be favored as means of reducing poverty. The detrimental effects of the oil palm expansion on a large set of ecosystem functions such as biodiversity, hydrological cycles and carbon storage are widely documented, as well as equity issues and land conflicts, posing serious threats to the long-term sustainability of oil palm. An assessment of the societal impact of oil palm needs to carefully weigh these different outcomes against each other. 


\subsection{Appendix}

\subsubsection{Supplementary figures}

Figure A1: Expansion of oil palm in Indonesia by producer type

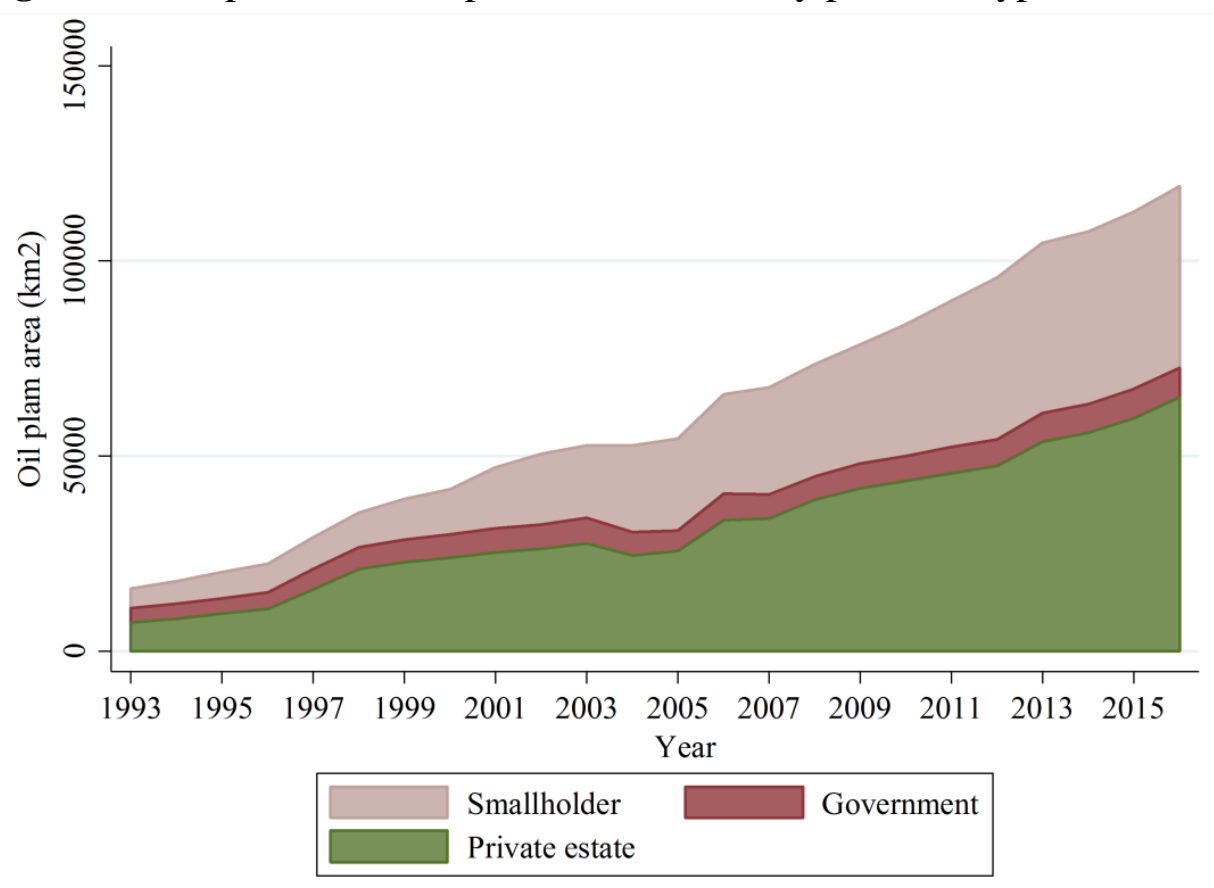

Source: Tree crop statistics.

Figure A2: Expansion of plantation crops in Indonesia

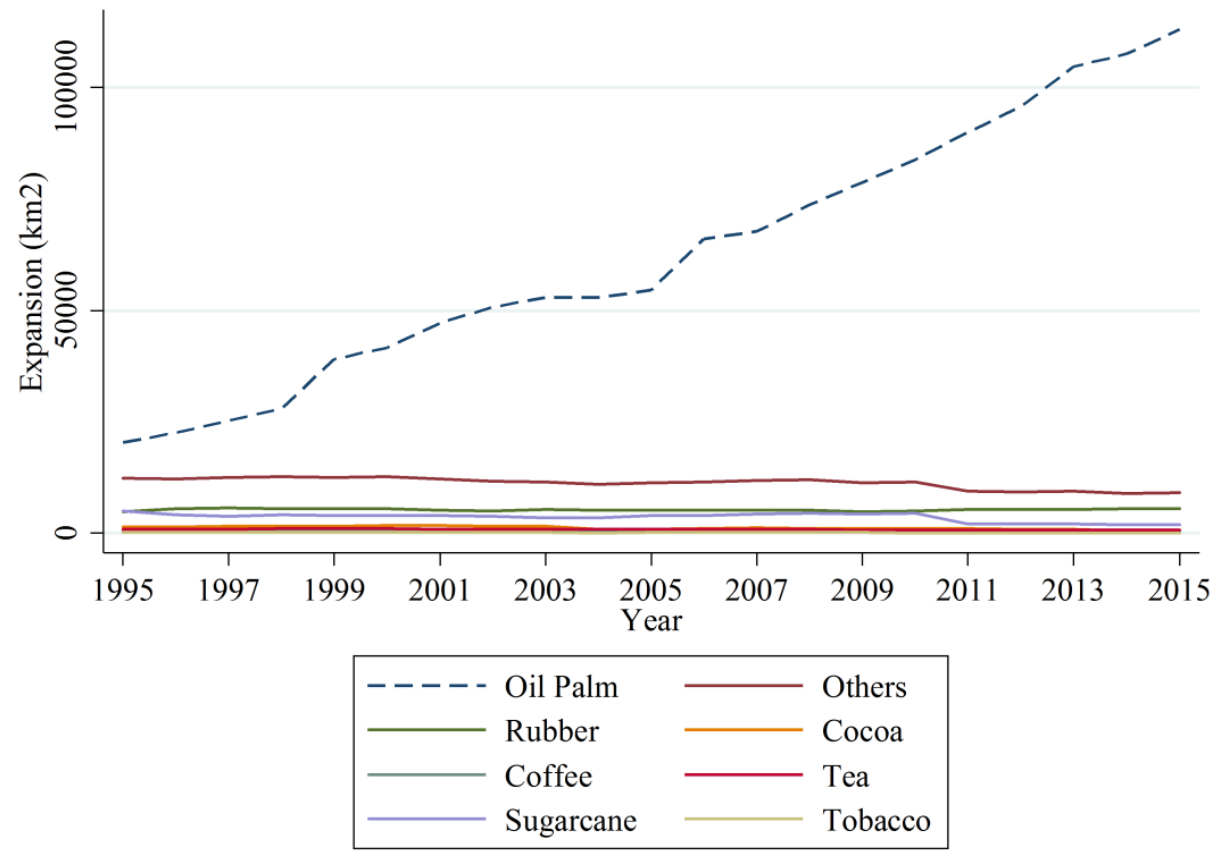

Source: Tree crop statistics. 
Figure A3: Effect of oil palm expansion on fertility using lags
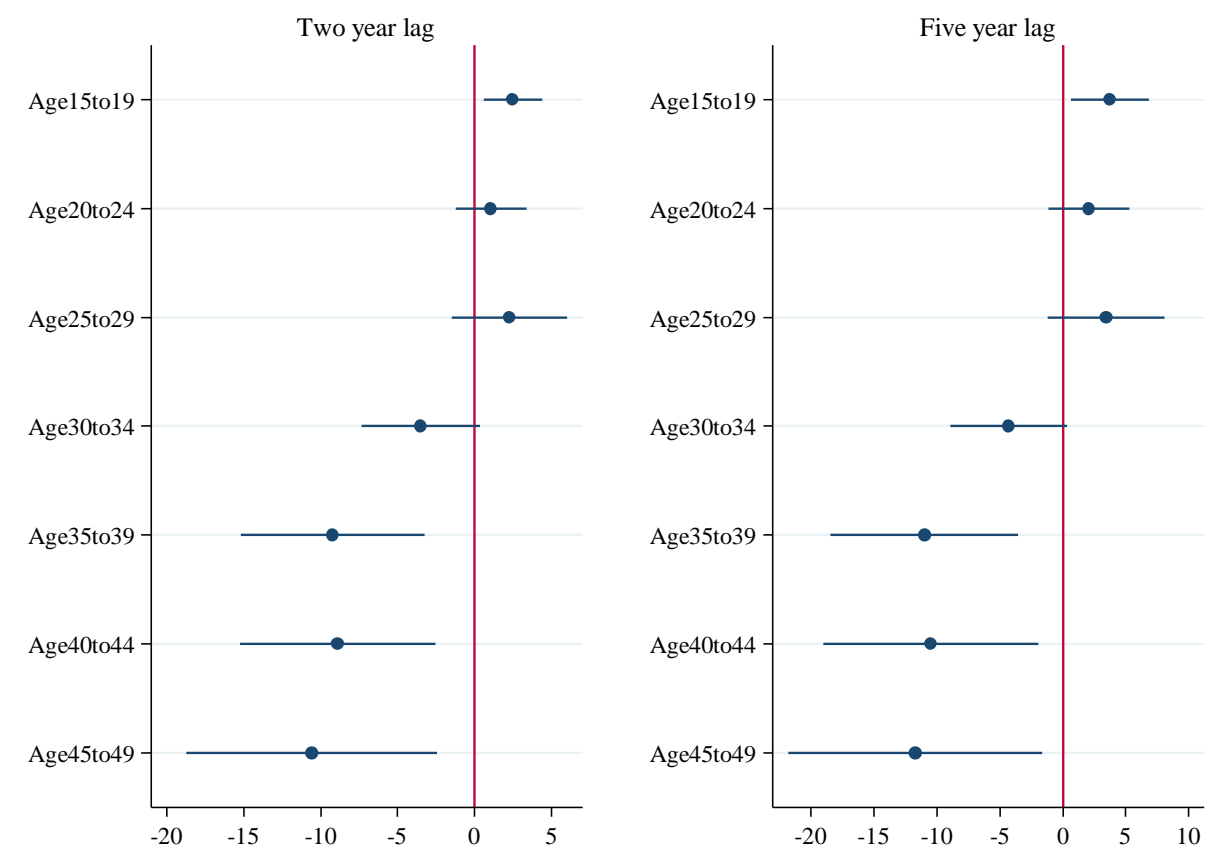

Notes: First figure uses a two year lag and second figure a five year lag for oil palm expansion both using the SUENAS data from 2001, 2006, 2011, 2016. IV estimates and 90\% CI are reported. All regressions control for national oil palm area, woman's age, regency and year fixed effects, island-year fixed effects, and initial values of fertility, electrification, share of agriculture in total employment and agricultural wages times year.

Figure A4: Effect of oil palm expansion on fertility using DHS data
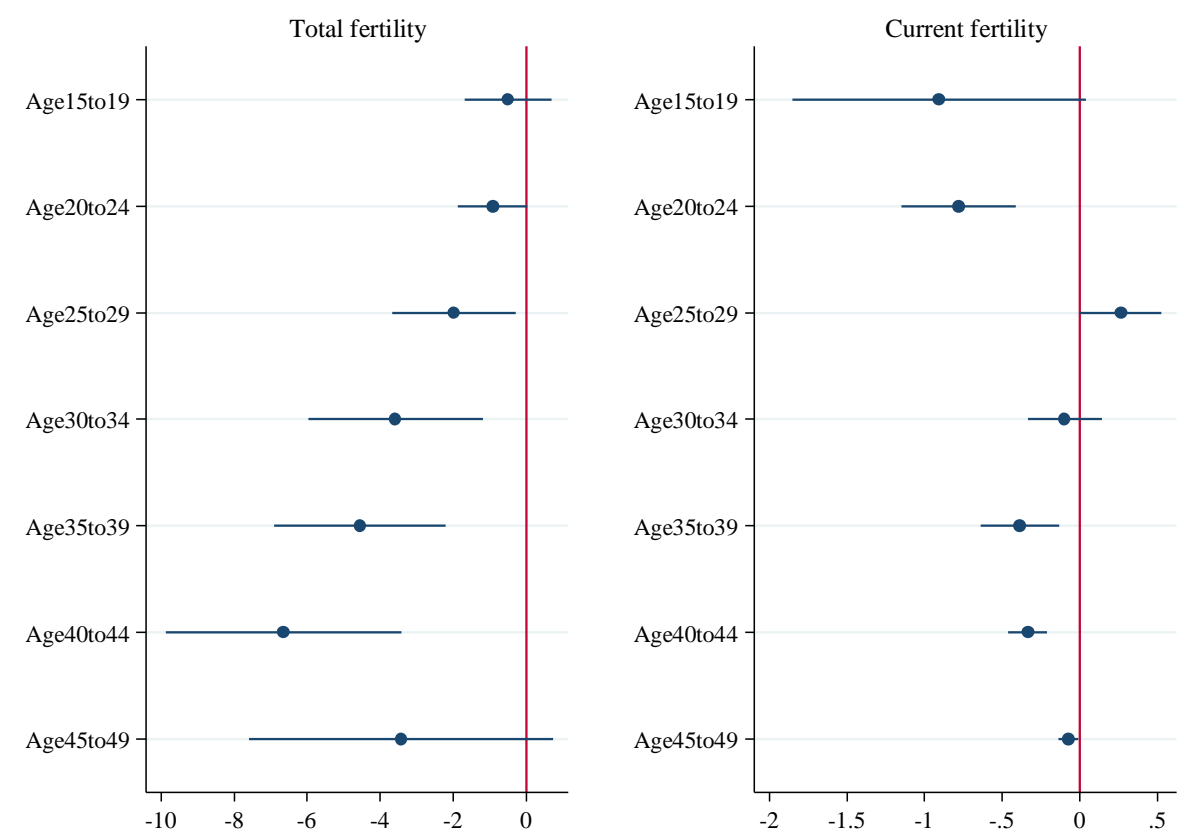

Notes: Marginal effects and 90\% CI are reported. All regressions include national oil palm area, province fixed effect and year dummies. 


\subsubsection{Supplementary tables}

Table A12: Description of data sources

\begin{tabular}{|c|c|c|}
\hline Data-source & Data availability & Description \\
\hline SUSENAS & $\begin{array}{l}1993,1996,2001 \\
2003,2006,2009 \\
2011,2013,2016\end{array}$ & $\begin{array}{l}\text { The National Socioeconomic Survey (SUSENAS) is a multi- } \\
\text { purpose socioeconomic survey at the individual level. The } \\
\text { SUSENAS is representative at the regency-level. }\end{array}$ \\
\hline PODES & $\begin{array}{l}1993,1996,2003, \\
2006,2014\end{array}$ & $\begin{array}{l}\text { The Village Potential Statistics (PODES) collects village and } \\
\text { urban neighborhood characteristics for all of Indonesia. }\end{array}$ \\
\hline SAKERNAS & $1993-2015$ & $\begin{array}{l}\text { The National Labor Force Survey (SAKERNAS) collects labor } \\
\text { market characteristics of working age individuals. } \\
\text { SAKERNAS is representative at the regency-level starting } \\
\text { from } 2007 \text {. }\end{array}$ \\
\hline GAEZ & 1960-1990 (baseline) & $\begin{array}{l}\text { The Global Agro-Ecological Zones (GAEZ) database provides } \\
\text { simulations on agro-climatic attainable yield and suitability } \\
\text { indices for crops under different conditions. }\end{array}$ \\
\hline $\begin{array}{l}\text { Tree Crop Statistics } \\
\& \\
\text { INDO-DAPOER }\end{array}$ & $\begin{array}{l}1967-2016 \\
\text { (national level) } \\
1996-2016 \\
\text { (regency level) }\end{array}$ & $\begin{array}{l}\text { The Indonesia Database for Policy and Economic Research } \\
\text { (INDO-DAPOER) and Tree Crop Statistics of the Ministry of } \\
\text { Agriculture provide data on smallholder oil palm area. Data is } \\
\text { available at the regency level starting 1996. Total oil palm area } \\
\text { at regency level is available since } 2005 \text {. }\end{array}$ \\
\hline DHS & $\begin{array}{l}1997,2002,2007 \\
2012\end{array}$ & $\begin{array}{l}\text { The Demographic and Health Survey (DHS) provides } \\
\text { representative data on health and population at the province } \\
\text { level, including details on child mortality, TFR, and number of } \\
\text { live births per woman. Proxies for female bargaining power } \\
\text { include the difference between actual and desired fertility, a } \\
\text { woman's influence on household and personal decisions and } \\
\text { her control over her own income. }\end{array}$ \\
\hline Indonesian Census & $\begin{array}{l}1995,2000,2005 \\
2010\end{array}$ & $\begin{array}{l}\text { From the IPUMS International database, we use the } 10 \% \\
\text { subsample of the Population Census of } 2000 \text { and } 2010 \text {, as well } \\
\text { as the } 0.43 \% \text { and } 0.51 \% \text { subsamples of the Intercensal } \\
\text { Population Surveys of } 1995 \text { and } 2005 \text {, respectively. We } \\
\text { calculate permanent migration as share of individuals aged } 15- \\
49 \text { who were born in a different regency than regency of } \\
\text { residence. Recent migration is the share of migrants who } \\
\text { moved to the regency in the last } 5 \text { years. The data is } \\
\text { aggregated at the regency level. }\end{array}$ \\
\hline $\begin{array}{l}\text { Badan Pusat Statistik } \\
\text { (BPS) }\end{array}$ & $1993-2016$ & $\begin{array}{l}\text { Rural and urban poverty lines are published annually in the } \\
\text { Statistic Year Book of Indonesia. Regional GDP by sector is } \\
\text { available since } 2000 \text {. }\end{array}$ \\
\hline Ministry of Finance & 1994- 2016 & $\begin{array}{l}\text { Public revenue data by source are available from the } \\
\text { Information System for Sub-National Budget. }\end{array}$ \\
\hline $\begin{array}{l}\text { NASA Shuttle Radar } \\
\text { Topographic Mission } \\
\text { (SRTM) }\end{array}$ & n.a. & $\begin{array}{l}\text { The SRTM digital elevation data is used to calculate average } \\
\text { altitude for each regency. }\end{array}$ \\
\hline
\end{tabular}


Table A13: Effect of oil palm expansion on fertility - 1993-2003

\begin{tabular}{lcccccc}
\hline & & \multicolumn{2}{c}{$\mathbf{1 9 9 3 - 2 0 0 3}$} & $(4)$ & $(5)$ & $(6)$ \\
& OLS & OLS & IV & IV & IV & IV \\
\hline Share of villages with & $-0.467^{* * * *}$ & $-0.315^{* *}$ & $-3.056^{* * * *}$ & $-5.143^{* * *}$ & $-2.804^{* * *}$ & $-1.902^{* *}$ \\
OP & $(0.117)$ & $(0.159)$ & $(0.736)$ & $(2.046)$ & $(1.052)$ & $(0.897)$ \\
& & & & & Yes \\
Regency \& year FE & Yes & Yes & Yes & Yes & Yes & Yes \\
Island-year FE & No & Yes & No & Yes & Yes & Yes \\
Initial levels * year & No & Yes & No & No & Yes & Yes \\
Woman's age & No & No & No & No & No & \\
& & & & 69.810 & 97.262 & 787.982 \\
F-stat & 371.677 & 154.411 & 148.439 & 8.905 & 10.664 & 10.663 \\
Kleibergen F-stat & & & 25.996 & 366496 & 366496 & 366496 \\
Observations & 366496 & 366496 & 366496 &
\end{tabular}

Table A14: Robustness checks

\begin{tabular}{|c|c|c|c|c|c|c|c|}
\hline & \multicolumn{5}{|c|}{ 1996-2006-2016 } & \multicolumn{2}{|c|}{ 2005-2011-2016 } \\
\hline & (1) & (2) & (3) & (4) & (5) & (6) & (7) \\
\hline & $\begin{array}{c}\text { No } \\
\text { weights }\end{array}$ & $\begin{array}{l}\text { Province } \\
\text { trends } \\
\text { included }\end{array}$ & $\begin{array}{l}\text { IV with } \\
\text { inter- } \\
\text { mediate } \\
\text { input level }\end{array}$ & $\begin{array}{c}\text { Excluding } \\
\text { Java }\end{array}$ & $\begin{array}{l}\text { Including } \\
\text { altitude } \mathrm{x} \\
\text { time trend }\end{array}$ & $\begin{array}{l}\text { Small- } \\
\text { holder OP } \\
\text { area }\end{array}$ & $\begin{array}{c}\text { Total OP } \\
\text { area }\end{array}$ \\
\hline Share of smallholder & $-6.256^{\text {***** }}$ & $-7.315^{* * *}$ & $-7.025^{* *}$ & $-4.354^{* *}$ & $-6.393^{*}$ & $-5.778^{*}$ & \\
\hline $\mathrm{OP}$ area in regency & $(2.419)$ & $(2.900)$ & $(2.900)$ & $(1.851)$ & (3.333) & $(3.484)$ & \\
\hline $\begin{array}{l}\text { Share of total OP } \\
\text { area in regency }\end{array}$ & & & & & & & $\begin{array}{l}-2.755^{*} \\
(1.548)\end{array}$ \\
\hline F-stat & 589.720 & 178.269 & 561.969 & 1033.998 & 567.656 & 313.331 & 311.721 \\
\hline Kleibergen F-stat & 12.389 & 8.603 & 10.586 & 15.711 & 7.719 & 9.815 & 18.993 \\
\hline Observations & 602758 & 602758 & 602758 & 332373 & 602758 & 658100 & 658100 \\
\hline
\end{tabular}

Table A15: Effect of oil palm expansion on fertility - Five year differences

\begin{tabular}{|c|c|c|c|c|c|c|}
\hline & \multicolumn{6}{|c|}{ 1996-2001-2006-2011-2016 } \\
\hline & (1) & (2) & (3) & (4) & (5) & (6) \\
\hline & OLS & OLS & IV & IV & IV & IV \\
\hline Share of smallholder & -0.214 & -0.304 & $-6.063^{* *}$ & $-14.005^{* *}$ & $-3.657^{* *}$ & $-6.642^{* *}$ \\
\hline $\mathrm{OP}$ area in regency & $(0.446)$ & $(0.414)$ & $(2.478)$ & (7.094) & $(1.808)$ & $(2.588)$ \\
\hline Regency \& year FE & Yes & Yes & Yes & Yes & Yes & Yes \\
\hline Island-year FE & No & Yes & No & Yes & Yes & Yes \\
\hline Initial levels * year & No & Yes & No & No & Yes & Yes \\
\hline Women's age & No & No & No & No & No & Yes \\
\hline F-stat & 226.705 & 564.779 & 148.756 & 100.667 & 465.440 & 521.266 \\
\hline Kleibergen F-stat & & & 15.046 & 7.482 & 9.719 & 9.719 \\
\hline Observations & 1006039 & 1006039 & 1006039 & 1006039 & 1006039 & 1006039 \\
\hline
\end{tabular}


Table A16: Transmission mechanisms - Child labor

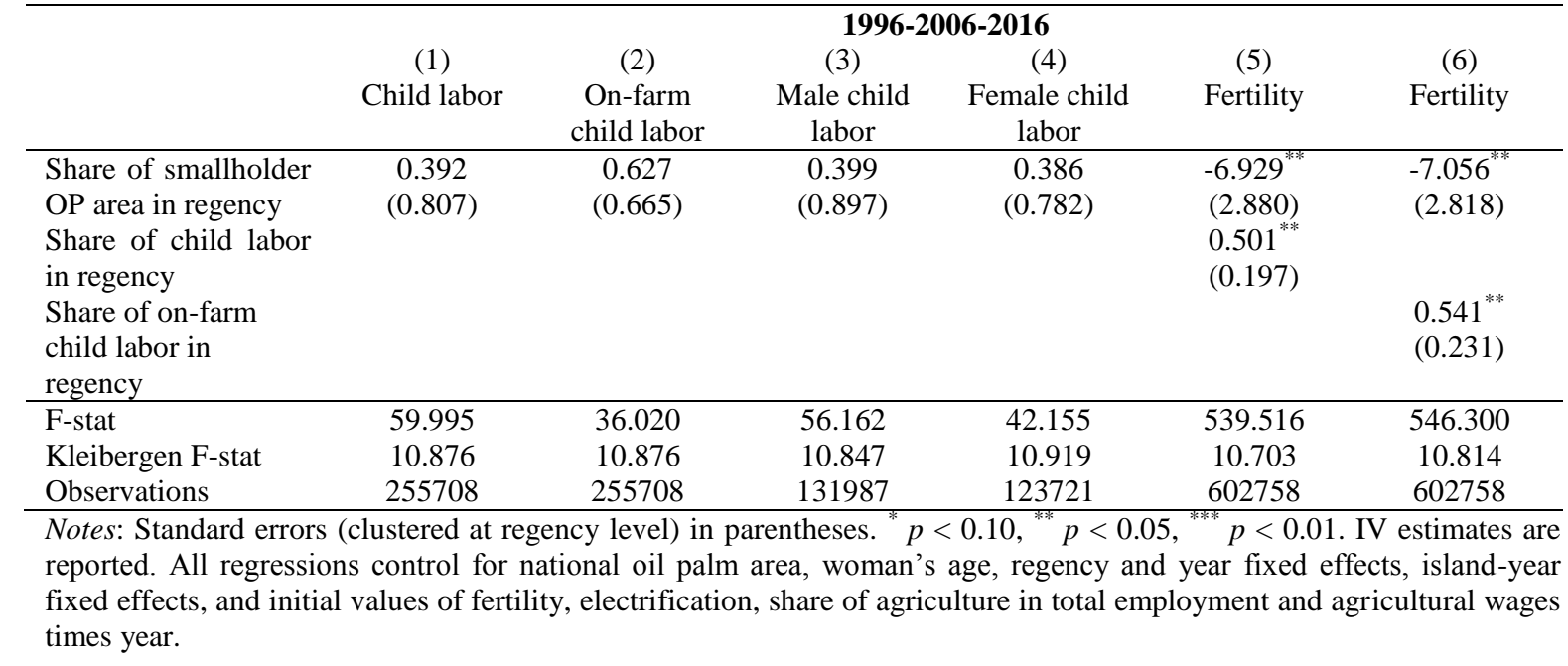

Table A17: Effect of oil palm on enrollment rates

\begin{tabular}{|c|c|c|c|}
\hline & $\begin{array}{c}\text { (1) } \\
\text { School enrollment } \\
\text { boys }(6-14 y)\end{array}$ & $\begin{array}{l}\text { 1996-2016-2016 } \\
(2) \\
\text { School enrollment } \\
\text { girls (6-14y) }\end{array}$ & $\begin{array}{c}\text { (3) } \\
\text { Fertility }\end{array}$ \\
\hline $\begin{array}{l}\text { Share of smallholder OP area in } \\
\text { regency }\end{array}$ & $\begin{array}{c}0.063 \\
(0.840)\end{array}$ & $\begin{array}{l}-0.271 \\
(0.789)\end{array}$ & $\begin{array}{l}-6.743^{* *} \\
(3.009)\end{array}$ \\
\hline $\begin{array}{l}\text { Share of 6-14 year olds enrolled } \\
\text { in regency }\end{array}$ & No & No & Yes \\
\hline F-stat & 123.715 & 121.733 & 475.657 \\
\hline Kleibergen F-stat & 10.915 & 10.872 & 11.188 \\
\hline Observations & 233283 & 219973 & 602758 \\
\hline
\end{tabular}


Table A18: Effect of oil palm on women's non-agricultural wages

\begin{tabular}{|c|c|c|c|c|c|c|c|}
\hline & $\begin{array}{c}(1) \\
\text { Women's } \\
\text { non-agr. wages }\end{array}$ & $\begin{array}{c}(2) \\
\text { Women's } \\
\text { non-agr. wages }\end{array}$ & $\begin{array}{c}(3) \\
\text { Women's } \\
\text { non-agr. wages }\end{array}$ & $\begin{array}{c}\text { 2001-2006-2011-2 } \\
(4) \\
\text { Women's } \\
\text { non-agr. wages }\end{array}$ & $\begin{array}{c}(5) \\
\text { Women's } \\
\text { non-agr. wages }\end{array}$ & $\begin{array}{c}(6) \\
\text { Women's } \\
\text { non-agr. wages }\end{array}$ & $\begin{array}{c}(7) \\
\text { Women's } \\
\text { non-agr. wages }\end{array}$ \\
\hline $\begin{array}{l}\text { Share of smallholder OP } \\
\text { in regency }(\%)\end{array}$ & $\begin{array}{l}16.127^{*} \\
(9.239)\end{array}$ & $\begin{array}{l}15.559^{*} \\
(9.019)\end{array}$ & $\begin{array}{l}15.810^{*} \\
(9.069)\end{array}$ & $\begin{array}{l}13.107 \\
(8.411)\end{array}$ & $\begin{array}{l}17.607^{*} \\
(9.383)\end{array}$ & $\begin{array}{l}14.535^{*} \\
(8.506)\end{array}$ & $\begin{array}{l}12.724 \\
(8.389)\end{array}$ \\
\hline $\begin{array}{l}\text { Women's educational } \\
\text { attainment }\end{array}$ & & $\begin{array}{l}0.301^{* * *} \\
(0.008)\end{array}$ & $\begin{array}{l}0.299^{* * *} \\
(0.010)\end{array}$ & & & & \\
\hline $\begin{array}{l}\text { Own revenue of } \\
\text { regency government }\end{array}$ & & & & $\begin{array}{l}0.772^{* * * *} \\
(0.084)\end{array}$ & & & $\begin{array}{l}0.752^{* * * *} \\
(0.088)\end{array}$ \\
\hline $\begin{array}{l}\text { Transfers from province } \\
\text { government }\end{array}$ & & & & & $\begin{array}{l}0.032^{* *} \\
(0.015)\end{array}$ & & \\
\hline $\begin{array}{l}\text { Transfers from central } \\
\text { government }\end{array}$ & & & & & $\begin{array}{c}0.825^{* * *} \\
(0.093)\end{array}$ & & \\
\hline $\begin{array}{l}\text { Average consumption } \\
\text { exp. p.c. in regency }\end{array}$ & & & & & & $\begin{array}{l}0.456^{* * *} \\
(0.090)\end{array}$ & $\begin{array}{c}0.132 \\
(0.090)\end{array}$ \\
\hline Sector dummies & No & No & Yes & No & No & No & No \\
\hline F-stat & 43.680 & 250.615 & 219.492 & 40.306 & 45.935 & 46.922 & 38.361 \\
\hline Kleibergen F-stat & 9.296 & 9.297 & 9.288 & 9.270 & 8.908 & 9.133 & 9.251 \\
\hline Observations & 72585 & 72585 & 72585 & 72505 & 72161 & 72585 & 72505 \\
\hline
\end{tabular}


Table A19: Effect of oil palm on sectoral shifts

\begin{tabular}{|c|c|c|c|c|c|c|c|c|}
\hline & \multicolumn{8}{|c|}{ 2001-2006-2011-2015 } \\
\hline & (1) & (2) & (3) & $(4)$ & (5) & (6) & (7) & (8) \\
\hline & $\begin{array}{c}\text { Female labor } \\
\text { force } \\
\text { participation }\end{array}$ & $\begin{array}{l}\text { Share of women } \\
\text { in non- } \\
\text { agricultural work }\end{array}$ & $\begin{array}{c}\text { Share of women } \\
\text { in agricultural } \\
\text { family work }\end{array}$ & $\begin{array}{l}\text { Share of women } \\
\text { in agricultural } \\
\text { wage work }\end{array}$ & $\begin{array}{l}\text { Male labor force } \\
\text { participation }\end{array}$ & $\begin{array}{l}\text { Share of men in } \\
\text { non-agricultural } \\
\text { work }\end{array}$ & $\begin{array}{l}\text { Share of men in } \\
\text { agricultural } \\
\text { family work }\end{array}$ & $\begin{array}{l}\text { Share of men in } \\
\text { agricultural wage } \\
\text { work }\end{array}$ \\
\hline Share of smallholder & -1.986 & 3.905 & $-5.181^{* *}$ & 1.308 & 0.212 & 0.751 & -1.235 & $2.865^{*}$ \\
\hline $\mathrm{OP}$ area in regency & (2.008) & $(2.476)$ & $(2.622)$ & $(1.077)$ & $(0.938)$ & (1.844) & $(1.020)$ & $(1.477)$ \\
\hline F-stat & 45.802 & 51.767 & 21.117 & 11.149 & 819.621 & 36.130 & 22.793 & 8.323 \\
\hline Kleibergen F-stat & 10.892 & 12.033 & 12.033 & 12.033 & 11.206 & 11.710 & 11.710 & 11.710 \\
\hline Observations & 371458 & 178948 & 178948 & 178948 & 367473 & 294740 & 294740 & 294740 \\
\hline
\end{tabular}

Notes: Standard errors (clustered at regency level) in parentheses. ${ }^{*} p<0.10,{ }^{* * *} p<0.05,{ }^{* * * *} p<0.01$. IV estimates are reported. All regressions control for national oil palm area, woman's age, regency and year fixed effects, island-year fixed effects, and initial values of fertility, electrification, share of agriculture in total employment and agricultural wages times year. 
Table A20: Effect of oil palm on wages - Returns to education

\section{1-2015}

(1) (2)

(3) (4)

(4) (5)

(6)

\begin{tabular}{lcccccc} 
& <Primary & $\begin{array}{c}\text { Women's wages } \\
\text { Secondary }\end{array}$ & Tertiary & <Primary & $\begin{array}{c}\text { Men's wages } \\
\text { Secondary }\end{array}$ & Tertiary \\
\hline Share of smallholder & -1.767 & 6.734 & $25.756^{* * *}$ & -2.231 & $12.638^{* *}$ & $29.466^{* *}$ \\
OP area in regency & $(5.318)$ & $(4.423)$ & $(9.804)$ & $(3.910)$ & $(6.340)$ & $(11.942)$ \\
\hline F-stat & 37.982 & 75.811 & 89.707 & 67.473 & 337.881 & 104.867 \\
Kleibergen F-stat & 9.136 & 7.974 & 9.885 & 8.666 & 8.221 & 8.646 \\
Observations & 137131 & 144666 & 61671 & 312686 & 375286 & 62201 \\
\hline
\end{tabular}

Notes: Standard errors (clustered at regency level) in parentheses. ${ }^{*} p<0.10,{ }^{* * *} p<0.05,{ }^{* * * *} p<0.01$. IV estimates are reported. All regressions control for national oil palm area, woman's age, regency and year fixed effects, island-year fixed effects, and initial values of fertility, electrification, share of agriculture in total employment and agricultural wages times year. All monetary values are in constant 1996 Indonesian Rupees. Wages are reported as log hourly wages. 2008 and 2013 data are not included due to inconsistent data for oil palm expansion.

Table A21: Transmission mechanisms - Returns to children's education II

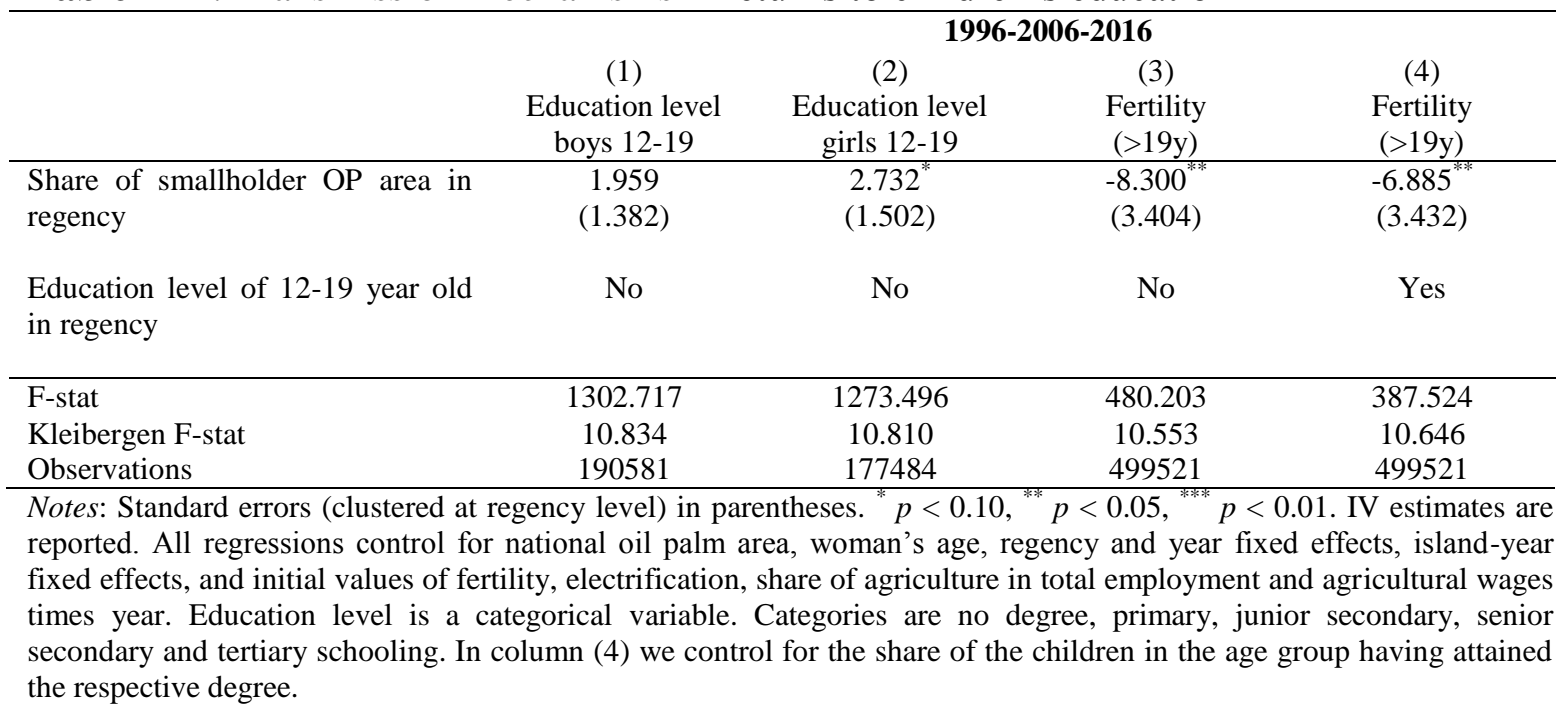

Table A22: Transmission mechanisms - Migration

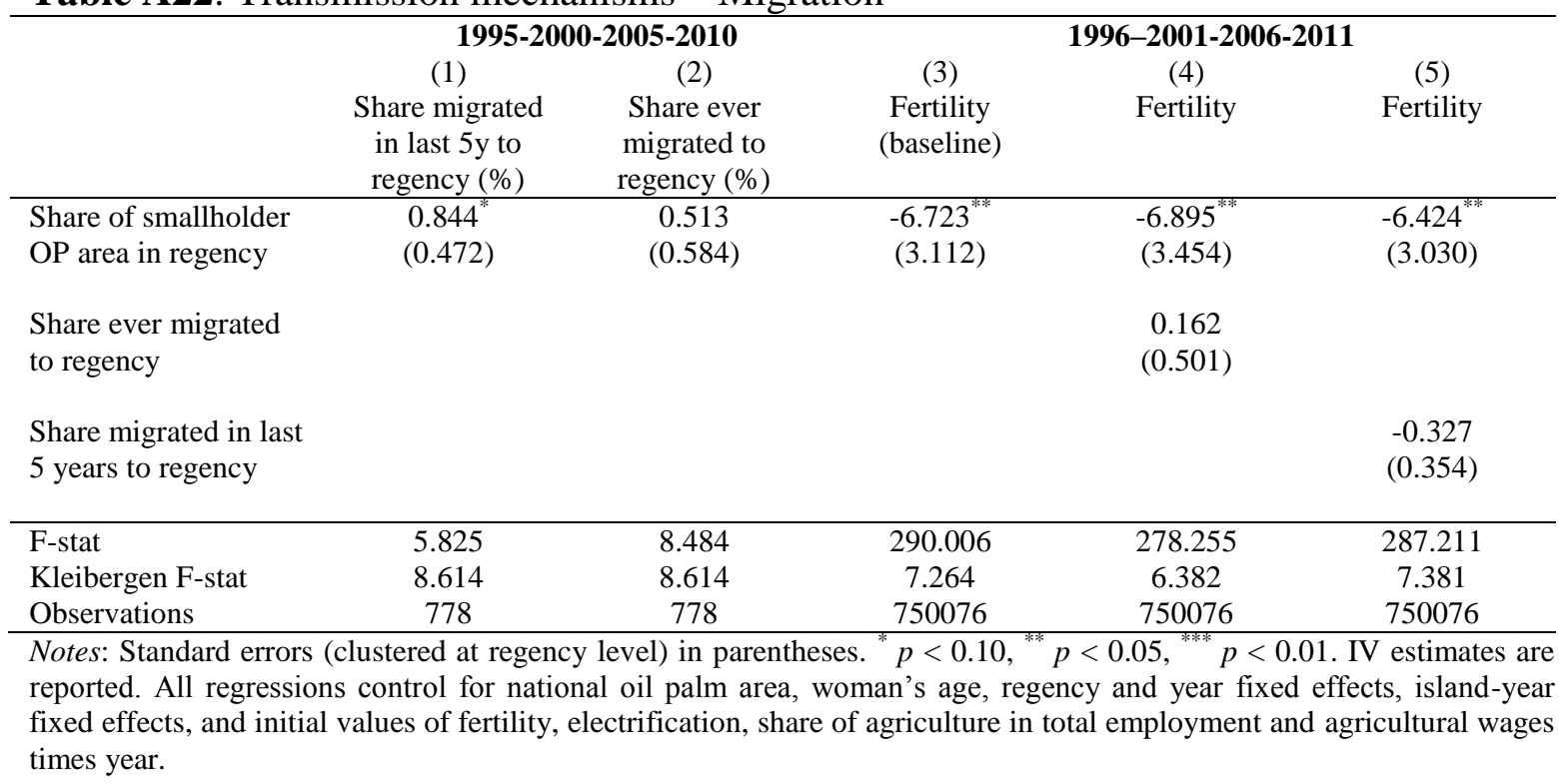


Table A23: Transmission mechanisms - Child mortality 1997-2002-2007-2012

1996-2001-2006-2011

(1)

Child mortality

(province level)

Share of smallholder OP

area in province/regency

$-109.888$

(55.954)

(2)

Fertility

(baseline)

$-7.477^{* *}$

(3.379)

(3)

Fertility

$-8.214^{* * *}$

(3.500)

Child mortality

(province level)

0.004

$(0.003)$

\begin{tabular}{lccc}
\hline F-stat & 6.824 & 302.543 & 286.543 \\
Kleibergen F-stat & & 7.460 & 8.389 \\
Observations & 80 & 794267 & 794267 \\
\hline
\end{tabular}

Notes: Standard errors (clustered at regency level) in parentheses. ${ }^{*} p<0.10,{ }^{* * *} p<0.05,{ }^{* * * *} p<0.01$. OLS estimates reported in col (1) and IV estimates reported in cols. (2) and (3). Column (1) controls for national oil palm area, province and year fixed effects. Columns (2) and (3) control for national oil palm area, woman's age, regency and year fixed effects, island-year fixed effects, and initial values of fertility, electrification, share of agriculture in total employment and agricultural wages times year.

Table A24: Transmission mechanisms - Female empowerment

1997-2002-2007-2012

1996-2006-2016

(DHS)

(1)

(2)

(3)

Fertility gap

Autonomy

Control over

(SUSENAS)

(4)

income

Share of

expenditures for food

\begin{tabular}{lcccc}
\hline $\begin{array}{l}\text { Share of smallholder OP } \\
\text { area in province/regency }\end{array}$ & -0.201 & 2.551 & 0.350 & -0.807 \\
\hline F-stat & $(0.951)$ & $(1.968)$ & $(0.706)$ & $(0.655)$ \\
$\begin{array}{l}\text { Kleibergen F-stat } \\
\text { Observations }\end{array}$ & 210.909 & 399.465 & 5.233 & 765.523 \\
\hline
\end{tabular}

Notes: Standard errors (clustered at province level in cols. (1) to (3) and at regency level in col. (4)) in parentheses. ${ }^{*} p<$ $0.10,{ }^{* *} p<0.05,{ }^{* * *} p<0.01$. OLS estimates reported in cols. (1) to (3) and IV estimates reported in col. (4). Columns (1) to (3) control for national oil palm area, province and year fixed effects. Column (4) controls for national oil palm area, woman's age, regency and year fixed effects, island-year fixed effects, and initial values of fertility, electrification, share of agriculture in total employment and agricultural wages times year as well as consumption expenditure. All monetary values are in $\log$ constant 1996 values. 
Table A25: Transmission mechanisms - Female empowerment II

\begin{tabular}{|c|c|c|c|c|c|c|}
\hline & $\begin{array}{l}\text { 2001-2006- } \\
2016\end{array}$ & & $01-2006-2$ & & $1996-20$ & $06-2011$ \\
\hline & $\begin{array}{c}(1) \\
\text { Fertility }\end{array}$ & $\begin{array}{c}(2) \\
\text { Fertility } \\
\text { (baseline) }\end{array}$ & $\begin{array}{c}\text { (3) } \\
\text { Fertility }\end{array}$ & $\begin{array}{c}(4) \\
\text { Fertility }\end{array}$ & $\begin{array}{c}(5) \\
\text { Fertility } \\
\text { (baseline) }\end{array}$ & $\begin{array}{c}(6) \\
\text { Fertility }\end{array}$ \\
\hline $\begin{array}{l}\text { Share of smallholder } \\
\text { OP area in regency }\end{array}$ & $\begin{array}{l}-4.935^{*} \\
(2.793)\end{array}$ & $\begin{array}{l}-5.501 \\
(4.201)\end{array}$ & $\begin{array}{l}-5.005 \\
(3.982)\end{array}$ & $\begin{array}{l}-6.628 \\
(4.713)\end{array}$ & $\begin{array}{l}-7.395^{* * * *} \\
(2.172)\end{array}$ & $\begin{array}{l}-8.644^{* * * * *} \\
(2.524)\end{array}$ \\
\hline $\begin{array}{l}\text { Share of expenditures } \\
\text { for food }\end{array}$ & $\begin{array}{l}-0.059^{*} \\
(0.032)\end{array}$ & & & & & \\
\hline $\begin{array}{l}\text { Consumption } \\
\text { expenditure p. c. }\end{array}$ & $\begin{array}{l}-0.519^{* * * *} \\
(0.014)\end{array}$ & & & & & \\
\hline Autonomy & & & $\begin{array}{l}0.167^{* *} \\
(0.078)\end{array}$ & & & \\
\hline Control over income & & & & $\begin{array}{l}-0.276^{*} \\
(0.153)\end{array}$ & & \\
\hline Fertility gap & & & & & & $\begin{array}{c}-0.222^{* * *} \\
(0.084)\end{array}$ \\
\hline F-stat & 640.976 & 240.163 & 254.516 & 250.456 & 319.620 & 292.408 \\
\hline Kleibergen F-stat & 10.580 & 5.254 & 5.375 & 4.928 & 17.236 & 16.586 \\
\hline Observations & 602758 & 531606 & 531606 & 531606 & 668945 & 668945 \\
\hline
\end{tabular}


Table A26: Additional summary statistics

Obs. Mean $\quad$ SD

SUSENAS (1996-2006-2016):

\section{Children (10-15y):}

Child labor $(=1)$

Child labor on own farm $(=1)$

$\begin{array}{lll}256332 & 0.059 & 0.236 \\ 256332 & 0.037 & 0.189 \\ & & \\ 233809 & 0.895 & 0.307 \\ 220537 & 0.902 & 0.297\end{array}$

Children (6-14y):

Enrollment rate boys

220537

0.297

SAKERNAS (2001-2006-2011-2015):

Individual level (15-49y):

Female labor force participation

Share of working women in non-agr. sector

$\begin{array}{lll}372425 & 0.511 & 0.500 \\ 179482 & 0.593 & 0.491 \\ 179482 & 0.274 & 0.446 \\ 179482 & 0.060 & 0.237 \\ 368477 & 0.841 & 0.365 \\ 295608 & 0.573 & 0.495 \\ 295608 & 0.079 & 0.270 \\ 295608 & 0.103 & 0.303\end{array}$

Share of working women in agr. sector (family work)

Share of working women in agr. sector (wage work)

Male labor force participation

Share of working men in non-agr. sector

Share of working men in agr. sector (family work)

Share of working men in agr. sector (wage work)

295608

0.103

0.303

\section{Regency level:}

Government revenue (mil. IDR)

Own revenue of regency government

832

36788.59

31317.13

Transfers from central government

832

29183

27551.28

Transfers from province government

832

4544.316

5122.167

DHS (1997-2002-2007-2012):

\section{Women (15-49y):}

Control over income $(=1)$

Autonomy index

$\begin{array}{lll}28836 & 0.650 & 0.477 \\ 73488 & 4.112 & 1.110 \\ 91330 & -0.534 & 1.642\end{array}$

Fertility gap

91330

1.642

Notes: All monetary values are in constant 1996 IDR. 


\section{General conclusion}

One of the most rapid changes in land use in recent decades was the expansion of oilproducing crops and the associated loss of tropical rainforest. While increasing production was necessary to meet the global demand for food, feed and fuel derived from oil crops, the associated loss and degradation of natural ecosystems sparked widespread concerns about the sustainability of oil crop cultivation. In particular, oil palm in Indonesia expanded rapidly due to its high land productivity compared to other oil crops. Oil palm covered approximately one million hectare in 1990 in Indonesia, rising to an estimated 12 million hectare in 2016 (Ministry of Agriculture 2017). It is widely documented that the oil palm expansion aggravated ecological hazards such as greenhouse gas emission and biodiversity loss through clearing tropical forest. Furthermore, oil palm expansion is also adversely affecting ecosystem functions through replacing less intensive agricultural production systems with higher levels of ecosystem functions. Yet, increasing evidence exists that oil palm cultivation contributed to welfare gains among smallholder farmers and beyond. These positive economic and negative environmental effects depict a strong tradeoff for local and global policy makers. Finding the right balance in policy-making requires a better understanding of the determinants of land-use change and its impacts in terms of various dimensions. This dissertation has contributed in these directions, analyzing landuse change and rural development in Indonesia with a particular focus on economic, institutional and demographic aspects of deforestation and oil palm expansion.

\subsection{Synopsis of three essays}

The dissertation consists of three substantive essays. The first essay has examined the linkages between secure property rights for agricultural land, agricultural productivity/intensity and potential outcomes for deforestation. We hypothesized that stronger land property rights could enable farmers to increase input intensity and productivity on the already cultivated land, thus reducing incentives to increase agricultural output by deforesting additional land. Our results show that formal land titles significantly increase agricultural intensity and productivity. However, due to land policy restrictions, 
farmers located at the historic forest margins are less likely to hold formal titles for the land they cultivate. We hypothesized that without land titles, these farmers are less able to intensify and might have been more likely to expand into the surrounding forest land to increase agricultural output. Indeed, historic forest closeness and past deforestation activities by households are found to be positively associated with current farm size. Our results suggest that the property right insecurity induced by the unregulated deforestation activities of farmers, combined with the fact that at forest margins farmers can more easily increase output by extending farm size via deforestation is highly problematic. The reason is that the incentive patterns induced by insecure property rights might even accelerate further deforestation. In addition to improving farmers' access to land titles for non-forest land, better recognition of customary land rights and more effective protection of forest land without recognized claims could be useful policy responses.

In the second essay, we have examined whether local farmers in Indonesia benefit from cultivating oil palm, examining also temporal variation in the welfare effect, and whether oil palm cultivation has spillover effects on other farm households. Using different regression models, we show that oil palm cultivation contributes to higher household consumption, measured in terms of expenditures on food and non-food goods and services. Oil palm has higher returns to labor than rubber, the main alternative cash crop in the region. However, returns to land are higher in rubber cultivation. The welfare gains are thus driven mainly by labor savings in oil palm cultivation. The lower labor requirements allow oil palm farmers to further expand their farmland and to reallocate saved labor to non-farm work. Due to declining rubber prices, the positive welfare effect of oil palm increased even more in 2015 compared to 2012. We do not find significant spillover effects of oil palm cultivation on neighboring farm households. Our results thus largely confirm the findings of the previous literature on the positive welfare effects of oil palm cultivation for smallholder farmers. Our findings also imply some important policy recommendations. First, policies aimed at regulating further oil palm expansion will have to account for the economic benefits for the local population. Second, part of the economic benefits of oil palm cultivation can be explained by labor savings in oil palm cultivation and the associated land expansion. To impede that land expansion leads to unregulated deforestation, it is of critical importance to improve forest and land governance. Since the economic gains of oil palm cultivation are substantial, small fines or low probabilities of 
conviction are unlikely to influence farmers' decision making and to deter further unregulated farm expansion. On the other side, high fines will adversely affect policy goals such as poverty reduction in rural areas. This suggests that improving governments' capacities to regulate land use, providing incentives for sustainable land use practices and alternative sources of income in rural areas might be more promising strategies.

While the introduction of new production technologies is often regarded as one of the key drivers of the historical fertility transition in the US and Western Europe, empirical evidence on the relationship between technology and fertility in a developing country context is largely inexistent. In the third essay, we address this gap by exploring why a labor-saving technology (crop choice) such as oil palm decreases fertility in rural Indonesia. Based on the current literature but also micro-level plot data, we show that oil palm indeed induces large labor savings per hectare compared to competing crops. We then use Becker's quantity-quality model to identify different causal mechanism through which the expansion of oil palm could affect fertility rates. While a labor-saving technology could theoretically increase fertility rates by decreasing maternal opportunity costs of time, we find consistently negative effects of the oil palm expansion on fertility. Our results suggest that income gains among agricultural households coupled with broader local economic development explain this effect. Specifically, local economic development seems to have raised returns to education and triggered investments into women's and children's education, which together with direct income effects explain the bulk of the negative effect of the oil palm expansion on fertility. From a policy perspective focused on female empowerment and reducing population growth, this indirect effect of the oil palm boom is certainly welcomed. However, to harness this effect more effectively, policies that improve female education and access to the non-agricultural employment are necessary. This does not mean that oil palm expansion is an effective instrument for rural development. The detrimental effects on ecosystem functions are widely documented as well as equity issues and land conflicts. Understanding the socioeconomic effects of the oil palm expansion may, however, contribute to design more sustainable agrarian systems. 


\subsection{Broader policy implications}

Overall, our research suggests that the oil palm expansion driven by smallholder farmers has contributed to rising human welfare in rural Indonesia. ${ }^{38}$ This finding is supported by our analyses based on both primary data from Sumatra and nationally representative data. However, current environmental research raises serious doubts on the sustainability of the oil palm boom. Policy interventions are in need to address this issue. While large-scale plantations are theoretically easy to regulate due to a limited number of actors and the implementation of sustainability standards such as RSPO (Roundtable on Sustainable Palm Oil), the smallholder sector is more complex to regulate. In principle, the strong involvement of smallholders in palm oil production is certainly important from a policy perspective focused on poverty reduction, since the welfare gains benefit households which are often among the poorest of society. Furthermore, if the growth in the agricultural sector is meant to be inclusive, it will be fundamental to integrate smallholders into sustainable value chains. Yet, our results show that large parts of the welfare gains of oil palm cultivation for smallholders run via labor savings and the subsequent reallocation of labor. Labor can be either allocated to additional farm land or to the non-farm sector. We have shown that the land and forest governance in Indonesia is rather weak, which could incentivize farmers to expand farm area at the expense of natural ecosystems. Besides improving land governance, it will hence be highly important to support farmers to reallocate their saved labor to the non-farm sector or to increase input intensity in a sustainable manner. This could include supporting the development of downstream industries in the palm oil sector as well as improving access to high quality seed and fertilizer, knowledge on sustainable management methods and secure land rights. Through creating additional jobs in the non-farm sector, potential adverse effects for women and landless households, who face low labor demand in oil palm cultivation, could also be addressed. We conclude that to manage the negative externalities of a labor-saving cropchoice such as oil palm, having secure property rights for agricultural land and forest as well as access to the non-agricultural sector might be particularly important. Future policy interventions should address these issues.

\footnotetext{
${ }^{38}$ Our data is based on oil palm expansion driven by the smallholder sector; welfare outcomes for the estate sector might be different.
} 


\subsection{Limitations and research gaps}

In the following, some limitations of this dissertation and gaps for potential future research are given. While the positive impact of stronger property rights on agricultural intensity and productivity are well established and our results support the findings in the literature, the effect of secure property rights for agricultural land on deforestation outcomes is more ambiguous due to data limitations. In particular, longer and spatially explicit panel data sets on land titling and deforestation patterns of smallholders are necessary to further confirm our findings. Research in this direction might be able to confirm if insecure property rights at the forest margins lead to cascade effects, accelerating deforestation and whether improved land and forest governance are able to stabilize forest margins. The Indonesian government is currently reforming their land and forest governance system, the potential effects on welfare and deforestation should be therefore of further interest.

Our analysis in the second essay focuses on determining the welfare effect of oil palm cultivation on farm households. However, a considerable part of households in many Indonesian villages identify as labor households without the necessary land to sustain them. Due to a combination of abundant labor supply and land scarcity, the increased labor productivity and savings in labor found in oil palm cultivation might have decreased the wages of laborers or restricted income gains to a small fraction of laborers. Although literature suggests that the average welfare effect on labor households might be positive (Edwards 2017), these findings might conceal considerable heterogeneity. Future research might also account for the long-term welfare effects of oil palm cultivation. Two issues might be in particular critical for long-term economic sustainability. First, ecosystem functions such as soil fertility, pollination and water supply have to stay at a sufficient level for oil palm cultivation. While increasing evidence exist regarding the adverse effects of oil palm expansion on hydrological cycles and soil fertility, oil palm was only recently introduced at a large scale and the full extent of production risk might only materialize in the long term. Second, prices for agricultural products fluctuated remarkably in recent decades, exposing small-scale farmers to a considerable price risk. The strong concentration of oil palm both at farm level and wider geographical scale and the limited possibility of adaption through changes in cropping patterns due to long growing periods and expensive planting are likely to exacerbate the risk. To avoid that fluctuation in 
income will lead to forced land sales or will drive households below the poverty line, access to credit and saving facilities to smooth consumption as well as access to alternative income sources might be crucial. Overall, understanding the effects of price risks and related uncertainties on farmers' livelihoods as well as designing respective countermeasures will be critical to support the long-term sustainability of smallholderdriven oil palm cultivation in Indonesia.

Concerning the effect of oil palm expansion on demographic variables such as fertility in the third essay, our results show a stable reducing effect on fertility. Yet, this effect may depend on several conditions such as accessible schooling, positive income effects and the growth potential of the non-agricultural sector as well as on specific changes in factor productivity. While we discuss these aspects, the evidence on the linkage between agricultural technologies on fertility is in general scarce, limiting the scope for comparisons. Future research might contribute to delineate how specific initial conditions and changes in factor productivity, shape the effect of new production technologies on fertility. Second, we have shown that oil palm expansion can lead to vast labor saving in particular for women and induces a shift from agriculture to the service sector. The implications of this effect reach certainly beyond fertility decisions. Women might have higher control over income and in general higher autonomy, which might change expenditure patterns and even political and social participation. We reported some average effects of oil palm expansion on female empowerment, however, the effects might be heterogeneous depending on the cultural context and access to non-agricultural employment. Furthermore, our proxies for female empowerment are aggregated at a high level. Detailed studies on gender-specific impacts of the oil palm expansion can be therefore promising. 


\section{References}

Abood, S. A.; Lee, J. S. H.; Burivalova, Z.; Garcia-Ulloa, J.; \& Koh, L. P. (2015). Relative Contributions of the Logging, Fiber, Oil Palm, and Mining Industries to Forest Loss in Indonesia. Conservation Letters 8 (1), 58-67.

Adhiati, A. S. M.; \& Bobsien, A. (2001). Indonesia's Transmigration programme - An Update. Available online at http://www.downtoearth-indonesia.org/sites/downtoearthindonesia.org/files/Transmigration\%20update\%202001.pdf, checked on 18.08.2016.

Ager, P.; Brueckner, M.; \& Herz, B. (2017). Structural Change and the Fertility Transition in the American South. (Discussion Papers on Business and Economics, 6). Odense: University of Southern Denmark.

Agrawal, A.; Chhatre, A.; \& Hardin, R. (2008). Changing governance of the world's forests. Science 320 (5882), 1460-1462.

Agris, J. von (2017). Rubber price drop, smallholder livelihood effects, and adaptation measures in Sumatra. Master's dissertation. Goettingen: University of Goettingen.

Alexandratos, N.; \& Bruinsma, J. (2012). World agriculture towards 2030/2050: The 2012 revision. (ESA Working paper, 12-03). Rome: Food and Agriculture Organization.

Amnesty International (2016). The great palm oil scandal: Labour abuses behind big brand namesAmnesty International. Available online at https://www.amnesty.org/download/Documents/ASA2152432016ENGLISH.PDF, checked on 17.05.2018.

Angelsen, A. \& Kaimowitz, D. (Eds.) (2001). Agricultural technologies and tropical deforestation. Wallingford (Oxfordshire) \& New York: CABI Pub.

Araujo, C.; Araujo Bonjean, C.; Combes, J.-L.; Combes Motel, P.; \& Reis, E. J. (2009). Property rights and deforestation in the Brazilian Amazon. Ecological Economics 68 (8-9), 2461-2468.

Atkin, D. (2009). Working for the Future: Female Factory Work and Child Health in Mexico. (Unpublished Manuscript). New Haven, USA: Yale University.

Azhar, B.; Lindenmayer, D. B.; Wood, J.; Fischer, J.; Manning, A.; McElhinny, C.; \& Zakaria, M. (2011). The conservation value of oil palm plantation estates, smallholdings and logged peat swamp forest for birds. Forest Ecology and Management 262 (12), 23062315 .

Badan Pusat Statistik (2012). Jambi in figures (Statistical Office of Indonesia). Jakarta, Indonesia. Available online at http://jambiprov.go.id/index.php?jbi_angka, checked on 02.02.2017.

Badan Pusat Statistik (2014). Poverty module: Social and population database. Jakarta, Indonesia. Available online at https://www.bps.go.id/subject/23/kemiskinan-danketimpangan.html\#subjekViewTab3, checked on 24.11.2014.

Baltagi, B. H. (Ed.) (2003). A companion to theoretical econometrics. Malden, Mass.: Blackwell. 
Banerjee, A. V.; Gertler, P. J.; \& Ghatak, M. (2002). Empowerment and efficiency: Tenancy reform in West Bengal. Journal of Political Economy 110 (2), 239-280.

Barbier, E. B.; \& Burgess, J. C. (1997). The economics of tropical forest land use options. Land Economics 73 (2), 174-195.

Barnes, A. D.; Jochum, M.; Mumme, S.; Haneda, N. F.; Farajallah, A.; Widarto, T. H.; $\&$ Brose, U. (2014). Consequences of tropical land use for multitrophic biodiversity and ecosystem functioning. Nature Communications 5, 5351-5357.

Barro, R. J. (1991). Economic Growth in a Cross Section of Countries. The Quarterly Journal of Economics 106 (2), 407-443.

Becker, G. S.; \& Lewis, G. (1973). On the Interaction between the Quantity and Quality of Children. Journal of Political Economy 81, 279-288.

Becker, G. S. (1981). A treatise on the family. Cambridge Mass. i.a.: Harvard University Press.

Becker, S. O.; Cinnirella, F.; \& Woessmann, L. (2010). The trade-off between fertility and education. Evidence from before the demographic transition. Journal of Economic Growth 15 (3), 177-204.

Belcher, B.; Imang, N.; \& Achdiawan, R. (2004). Rattan, Rubber, or Oil Palm. Cultural and Financial Considerations for Farmers in Kalimantan. Economic Botany 58 (sp1), 7787.

Bellemare, M. F. (2013). The productivity impacts of formal and informal land rights: Evidence from Madagascar. Land Economics 89 (2), 272-290.

Besley, T. (1995). Property rights and investment incentives: Theory and evidence from Ghana. Journal of Political Economy 103 (5), 903-937.

Black, S. E.; Devereux, P. J.; \& Salvanes, K. G. (2005). The More the Merrier? The Effect of Family Size and Birth Order on Children's Education. The Quarterly Journal of Economics 120 (2), 669-700.

Bleakley, H.; \& Lange, F. (2009). Chronic Disease Burden and the Interaction of Education, Fertility, and Growth. Review of Economics and Statistics 91 (1), 52-65.

Blundell, R.; \& Preston, I. (1998). Consumption inequality and income uncertainty. The Quarterly Journal of Economics 113 (2), 603-640.

Bongaarts, J. (1992). Population Growth and Global Warming. Population and Development Review 18 (2), 299-319.

Bou Dib, J.; Krishna, V. V.; Alamsyah, Z.; \& Qaim, M. (2018). Land-use change and livelihoods of non-farm households: The role of income from employment in oil palm and rubber in rural Indonesia. (EFForTS Discussion Paper, 21). Goettingen: University of Goettingen.

Bourke, R. M. \& Harwood, T. (Eds.) (2009). Food and Agriculture in Papua New Guinea. Canberra: ANU E Press.

Brasselle, A.-S.; Gaspart, F.; \& Platteau, J.-P. (2002). Land tenure security and investment incentives: puzzling evidence from Burkina Faso. Journal of Development Economics 67 (2), 373-418. 
Bren d'Amour, C.; Reitsma, F.; Baiocchi, G.; Barthel, S.; Guneralp, B.; Erb, K.-H. et al. (2016). Future urban land expansion and implications for global croplands. Proceedings of the National Academy of Sciences 114 (34), 8939-8944.

Budidarsono, S.; Dewi, S.; Sofiyuddin, M.; \& Rahmanulloh, A. (2012). SocioEconomic Impact Assessment of Palm Oil Production. (Technical Brief, 24). Bogor, Indonesia: World Agroforestry Centre (ICRAF), SEA Regional Office.

Burney, J. A.; Davis, S. J.; \& Lobell, D. B. (2010). Greenhouse gas mitigation by agricultural intensification. Proceedings of the National Academy of Sciences 107 (26), $12052-12057$.

Butler, R. A.; \& Laurance, W. F. (2009). Is oil palm the next emerging threat to the Amazon? Tropical Conservation Science 2 (1), 1-10.

Byerlee, D.; Falcon, W. P.; \& Naylor, R. (2017). The tropical oil crop revolution. Food, feed, fuel, and forests. New York, USA: Oxford University Press.

Cacho, O. J.; Milne, S.; Gonzalez, R.; \& Tacconi, L. (2014). Benefits and costs of deforestation by smallholders. Implications for forest conservation and climate policy. Ecological Economics 107, 321-332.

Cameron, A. C.; Gelbach, J. B.; \& Miller, D. L. (2011). Robust inference with multiway clustering. Journal of Business \& Economic Statistics 29 (2), 238-249.

Campbell, O.; \& Graham, W. J. (2006). Strategies for reducing maternal mortality. Getting on with what works. The Lancet 368 (9543), 1284-1299.

Carlson, K. M.; Curran, L. M.; Ratnasari, D.; Pittman, A. M.; Soares-Filho, B. S.; Asner, G. P. et al. (2012). Committed carbon emissions, deforestation, and community land conversion from oil palm plantation expansion in West Kalimantan, Indonesia. Proceedings of the National Academy of Sciences 109 (19), 7559-7564.

Chen, L. C.; Gesche, M. C.; Ahmed, S.; Chowdhury, A. I.; \& Mosley, W. H. (1974). Maternal Mortality in Rural Bangladesh. Studies in Family Planning 5 (11), 334-341.

Cleland, J.; \& Wilson, C. (1987). Demand Theories of the Fertility Transition: An Iconoclastic View. Population Studies 41 (1), 5-30.

Clough, Y.; Krishna, V. V.; Corre, M. D.; Darras, K.; Denmead, L. H.; Meijide, A. et al. (2016). Land-use choices follow profitability at the expense of ecological functions in Indonesian smallholder landscapes. Nature Communications 7, 13137-13149.

Corrado, L.; \& Fingleton, B. (2012). Where is the Economics in Spatial Econometrics? Journal of Regional Science 52 (2), 210-239.

Cramb, R.; \& McCarthy, J. F. (2016a). Characterising oil palm production in Indonesia and Malaysia. In Cramb, R.; \& McCarthy, J. F. (Eds.): The oil palm complex: Smallholders, agribusiness, and the state in Indonesia and Malaysia. Singapore: NUS Press, 27-77.

Cramb, R. \& McCarthy, J. F. (Eds.) (2016b). The oil palm complex: Smallholders, agribusiness, and the state in Indonesia and Malaysia. Singapore: NUS Press.

Damnyag, L.; Saastamoinen, O.; Appiah, M.; \& Pappinen, A. (2012). Role of tenure insecurity in deforestation in Ghana's high forest zone. Forest Policy and Economics 14 (1), 90-98. 
Deininger, K.; \& Feder, G. (2001). Land institutions and land markets. In Gardener, B.; \& Rausser, G. (Eds.): Handbook of Agricultural Economics. Amsterdam: Elsevier, 288331.

Deininger, K.; Ali, D. A.; \& Alemu, T. (2011). Impacts of land certification on tenure security, investment, and land market participation: Evidence from Ethiopia. Land Economics 87 (2), 312-334.

Dietz, T.; \& Rosa, E. A. (1997). Effects of population and affluence on CO2 emissions. Proceedings of the National Academy of Sciences 94 (1), 175-179.

Dislich, C.; Keyel, A. C.; Salecker, J.; Kisel, Y.; Meyer, K. M.; Auliya, M. et al. (2017). A review of the ecosystem functions in oil palm plantations, using forests as a reference system. Biological Reviews 92 (3), 1539-1569.

Doepke, M. (2004). Accounting for Fertility Decline During the Transition to Growth. Journal of Economic Growth 9 (3), 347-383.

Drescher, J.; Rembold, K.; Allen, K.; Beckschafer, P.; Buchori, D.; Clough, Y. et al. (2016). Ecological and socio-economic functions across tropical land use systems after rainforest conversion. Philosophical transactions of the Royal Society of London. Series B, Biological sciences 371 (1694), p. 20150275.

Duflo, E.; \& Pande, R. (2007). Dams. The Quarterly Journal of Economics 122 (2), 601-646.

Edwards, R. (2017). Tropical Oil Crops and Rural Poverty. Stanford University: Department of Earth System Science and the Center on Food Security and the Environment.

EFForTs-Information System: University of Goettingen (2017). Available online at https://efforts-is.uni-goettingen.de, checked on 25.09.2017.

Elhorst, J. P. (2010). Applied Spatial Econometrics. Raising the Bar. Spatial Economic Analysis 5 (1), 9-28.

Euler, M.; Hoffmann, M. P.; Fathoni, Z.; \& Schwarze, S. (2016a). Exploring yield gaps in smallholder oil palm production systems in eastern Sumatra, Indonesia. Agricultural Systems 146, 111-119.

Euler, M.; Schwarze, S.; Siregar, H.; \& Qaim, M. (2016b). Oil palm expansion among smallholder farmers in Sumatra, Indonesia. Journal of Agricultural Economics 67 (3), 658-676.

Euler, M.; Krishna, V. V.; Schwarze, S.; Siregar, H.; \& Qaim, M. (2017). Oil palm adoption, household welfare, and nutrition among smallholder farmers in Indonesia. World Development 93, 219-235.

Ewers, R. M.; Scharlemann, J. P. W.; Balmford, A.; \& Green, R. E. (2009). Do increases in agricultural yield spare land for nature? Global Change Biology 15 (7), 17161726.

FAO (2016). Global forest resources assessment 2015. How are the world's forests changing? 2nd Edition. Rome: Food and Agriculture Organization.

FAOSTAT (2014). Production. Rome: Food and Agricultural Organization. Available online at http://faostat3.fao.org., checked on July 2017. 
Fearnside, P. M. (2005). Deforestation in Brazilian Amazonia: History, rates, and consequences. Conservation Biology 19 (3), 680-688.

Feder, G.; \& Feeny, D. (1991). Land tenure and property rights: Theory and implications for development policy. The World Bank Economic Review 5 (1), 135-153.

Feintrenie, L.; Chong, W. K.; \& Levang, P. (2010). Why do Farmers Prefer Oil Palm? Lessons Learnt from Bungo District, Indonesia. Small-scale Forestry 9 (3), 379-396.

Fenske, J. (2011). Land tenure and investment incentives. Evidence from West Africa. Journal of Development Economics 95 (2), 137-156.

Fernihough, A. (2017). Human capital and the quantity-quality trade-off during the demographic transition. Journal of Economic Growth 22 (1), 35-65.

Filmer, D.; \& Pritchett, L. H. (2001). Estimating wealth effects without expenditure data - or tears: An application to educational enrollments in states of India. Demography 38 (1), 115-132.

Fischer, G.; Nachtergaele, F. O.; Prieler, S.; Teixeira, E.; Tóth, G.; van Velthuizen, H. et al. (2012). Global Agro-Ecological Zones (GAEZ v3.0): Model Documentation. Laxenburg, Austria and Rome, Italy: IIASA and FAO.

Fitzherbert, E. B.; Struebig, M. J.; Morel, A.; Danielsen, F.; Bruhl, C. A.; Donald, P. F.; $\&$ Phalan, B. (2008). How will oil palm expansion affect biodiversity? Trends in ecology \& evolution 23 (10), 538-545.

Foley, J. A.; Defries, R.; Asner, G. P.; Barford, C.; Bonan, G.; Carpenter, S. R. et al. (2005). Global consequences of land use. Science 309 (5734), 570-574.

Galor, O.; \& Weil, D. N. (2000). Population, Technology, and Growth. From Malthusian Stagnation to the Demographic Transition and Beyond. American Economic Review 90 (4), 806-828.

Ganser, D.; Denmead, L. H.; Clough, Y.; Buchori, D.; \& Tscharntke, T. (2017). Local and landscape drivers of arthropod diversity and decomposition processes in oil palm leaf axils. Agricultural and Forest Entomology 19 (1), 60-69.

Gatto, M.; Wollni, M.; \& Qaim, M. (2015). Oil palm boom and land-use dynamics in Indonesia. The role of policies and socioeconomic factors. Land Use Policy 46, 292-303.

Gatto, M.; Wollni, M.; Asnawi, R.; \& Qaim, M. (2017). Oil palm boom, contract farming, and rural economic development. Village-level evidence from Indonesia. World Development 95, 127-140.

Gibbs, H. K.; Ruesch, A. S.; Achard, F.; Clayton, M. K.; Holmgren, P.; Ramankutty, N.; \& Foley, J. A. (2010). Tropical forests were the primary sources of new agricultural land in the 1980s and 1990s. Proceedings of the National Academy of Sciences 107 (38), 16732-16737.

Godar, J.; Gardner, T. A.; Tizado, E. J.; \& Pacheco, P. (2014). Actor-specific contributions to the deforestation slowdown in the Brazilian Amazon. Proceedings of the National Academy of Sciences 111 (43), 15591-15596.

Goldstein, M.; \& Udry, C. (2008). The profits of power: Land rights and agricultural investment in Ghana. Journal of Political Economy 116 (6), 981-1022. 
Green, R. E.; Cornell, S. J.; Scharlemann, J. P. W.; \& Balmford, A. (2005). Farming and the fate of wild nature. Science 307 (5709), 550-555.

Grimm, M.; \& Klasen, S. (2015). Migration pressure, tenure security, and agricultural intensification: Evidence from Indonesia. Land Economics 91 (3), 411-434.

Groot, R. S. de (1992). Functions of nature. Evaluation of nature in environmental planning. management and decision making. Groningen: Wolters-Noordhoff.

Guillaume, T.; Damris, M.; \& Kuzyakov, Y. (2015). Losses of soil carbon by converting tropical forest to plantations: erosion and decomposition estimated by delta(13) C. Global Change Biology 21 (9), 3548-3560.

Guillaume, T.; Holtkamp, A. M.; Damris, M.; Brümmer, B.; \& Kuzyakov, Y. (2016). Soil degradation in oil palm and rubber plantations under land resource scarcity. Agriculture, Ecosystems \& Environment 232, 110-118.

Guinnane, T. W. (2011). The Historical Fertility Transition: A Guide for Economists. Journal of Economic Literature 49 (3), 589-614.

Halleck Vega, S.; \& Elhorst, J. P. (2015). The SLX Model. Journal of Regional Science 55 (3), 339-363.

Hausman, J. A. (1978). Specification tests in econometrics. Econometrica 46, 12511271.

Heath, R.; \& Mobarak, M. A. (2015). Manufacturing growth and the lives of Bangladeshi women. Journal of Development Economics 115, 1-15.

Hoffmann, M. P.; Donough, C. R.; Cook, S. E.; Fisher, M. J.; Lim, C. H.; Lim, Y. L. et al. (2017). Yield gap analysis in oil palm: Framework development and application in commercial operations in Southeast Asia. Agricultural Systems 151, 12-19.

Holden, S. T.; Deininger, K.; \& Ghebru, H. (2009). Impacts of low-cost land certification on investment and productivity. American Journal of Agricultural Economics 91 (2), 359-373.

Indrarto, G. B.; Murharjanti, P.; Khatarina, J.; Pulungan, I.; Ivalerina, F.; Rahman, J. et al. (2012). The context of REDD+ in Indonesia. Bogor, Indonesia: Center for International Forestry Research.

Jacoby, H. G.; \& Minten, B. (2007). Is land titling in Sub-Saharan Africa costeffective? Evidence from Madagascar. The World Bank Economic Review 21 (3), 461-485.

Kirk, D. (1996). Demographic transition theory. Population Studies 50 (3), 361-387.

Koczberski, G. (2007). Loose Fruit Mamas. Creating Incentives for Smallholder Women in Oil Palm Production in Papua New Guinea. World Development 35 (7), 1172 1185 .

Koh, L. P.; Miettinen, J.; Liew, S. C.; \& Ghazoul, J. (2011). Remotely sensed evidence of tropical peatland conversion to oil palm. Proceedings of the National Academy of Sciences 108 (12), 5127-5132.

Komarudin, H.; Siagian, Y.; \& Colfer, C. (2008). Collective action to secure property rights for the poor. A case study in Jambi Province, Indonesia. (CAPRi Working Paper, 90). Washington, DC: CAPRi (CGIAR). 
Konopik, O.; Steffan-Dewenter, I.; \& Grafe, T. U. (2015). Effects of logging and oil palm expansion on stream frog communities on Borneo, Southeast Asia. Biotropica 47 (5), 636-643.

Kopp, T.; \& Brümmer, B. (2017). Traders' market power along Indonesian rubber value chains. China Agricultural Economic Review 9 (2), 169-187.

Krishna, V. V.; Euler, M.; Siregar, H.; \& Qaim, M. (2017a). Differential livelihood impacts of oil palm expansion in Indonesia. Agricultural Economics 48 (5), 639-653.

Krishna, V. V.; Kubitza, C.; Pascual, U.; \& Qaim, M. (2017b). Land markets, property rights, and deforestation: Insights from Indonesia. World Development 99, 335-349.

Kulu, H. (2005). Migration and Fertility. Competing Hypotheses Re-examined. European Journal of Population / Revue européenne de Démographie 21 (1), 51-87.

Kunz, Y.; Hein, J.; Mardiana, R.; \& Faust, H. (2016). Mimicry of the Legal: Translating de jure Land Formalization Processes Into de facto Local Action in Jambi province, Sumatra. Austrian Journal of South-East Asian Studies 9 (1), 127-146.

Lambin, E. F.; \& Meyfroidt, P. (2011). Global land use change, economic globalization, and the looming land scarcity. Proceedings of the National Academy of Sciences 108 (9), 3465-3472.

LANDac (2016). Food security and land governance fact sheet - Indonesia. Available online at http://www.landgovernance.org/resources/indonesia-factsheet-2012/, checked on 05.09.2017.

Laurance, W. F.; Sayer, J.; \& Cassman, K. G. (2014). Agricultural expansion and its impacts on tropical nature. Trends in ecology \& evolution 29 (2), 107-116.

Lawry, S.; Samii, C.; Hall, R.; Leopold, A.; Hornby, D.; \& Mtero, F. (2016). The impact of land property rights interventions on investment and agricultural productivity in developing countries. A systematic review. Journal of Development Effectiveness, 1-21.

Lee, J. S. H.; Abood, S. A.; Ghazoul, J.; Barus, B.; Obidzinski, K.; \& Koh, L. P. (2014). Environmental Impacts of Large-Scale Oil Palm Enterprises Exceed that of Smallholdings in Indonesia. Conservation Letters 7 (1), 25-33.

Lee, R.; \& Mason, Andrew (2006). What Is the Demographic Dividend? Finance and Development 43, 16-17.

LeSage, J. P.; \& Pace, R. K. (2009). Introduction to spatial econometrics. Boca Raton: CRC Press (Statistics, textbooks and monographs, 196).

Levy, V. (1985). Cropping Pattern, Mechanization, Child Labor, and Fertility Behavior in a Farming Economy. Rural Egypt. Economic Development and Cultural Change 33 (4), 777-791.

Lewis, D. J.; Barham, B. L.; \& Robinson, B. (2011). Are There Spatial Spillovers in the Adoption of Clean Technology? The Case of Organic Dairy Farming. Land Economics 87 (2), 250-267.

Liscow, Z. D. (2013). Do property rights promote investment but cause deforestation? Quasi-experimental evidence from Nicaragua. Journal of Environmental Economics and Management 65 (2), 241-261. 
Li, T. M. (2015). Social impacts of oil palm in Indonesia. A gendered perspective from West Kalimantan. Bogor, Indonesia: Center for International Forestry Research (CIFOR).

Lucey, J. M.; \& Hill, J. K. (2012). Spillover of Insects from Rain Forest into Adjacent Oil Palm Plantations. Biotropica 44 (3), 368-377.

Luck, G. W. (2007). A review of the relationships between human population density and biodiversity. Biological Reviews 82 (4), 607-645.

Luskin, M. S.; Christina, E. D.; Kelley, L. C.; \& Potts, M. D. (2014). Modern Hunting Practices and Wild Meat Trade in the Oil Palm Plantation-Dominated Landscapes of Sumatra, Indonesia. Human Ecology 42 (1), 35-45.

Maertens, M.; Zeller, M.; \& Birner, R. (2006). Sustainable agricultural intensification in forest frontier areas. Agricultural Economics 34 (2), 197-206.

Margono, B. A.; Potapov, P. V.; Turubanova, S.; Stolle, F.; \& Hansen, M. C. (2014). Primary forest cover loss in Indonesia over 2000-2012. Nature Climate Change 4 (8), 730-735.

Melati, D. N.; Jaya, I. N. S.; Zuhdi, M.; Pérez-Cruzado, C.; Fehrmann, L.; \& Kleinn, C. (2014). Remote sensing based monitoring of land transformation in Jambi Province, Sumatra. In Kleinn, C.; Kleinn, A.; \& Fehrmann, L. (Eds.): The ecological and economic challenges of managing forested landscapes in global context. Goettingen: Cuvillier Verlag.

Merten, J.; Röll, A.; Guillaume, T.; Meijide, A.; Tarigan, S.; Agusta, H. et al. (2016). Water scarcity and oil palm expansion. Social views and environmental processes. Ecology and Society 21 (2).

Meyfroidt, P.; Vu, T. P.; \& Hoang, V. A. (2013). Trajectories of deforestation, coffee expansion and displacement of shifting cultivation in the Central Highlands of Vietnam. Global Environmental Change 23 (5), 1187-1198.

Miller, G. (2010). Contraception as Development? New Evidence from Family Planning in Colombia. The Economic Journal 120 (545), 709-736.

Ministry of Agriculture (2017). Basis Data Statistik Pertanian (BDSP). Available online at http://en.litbang.pertanian.go.id/, checked on 25.07.2017.

Munshi, K.; \& Myaux, J. (2006). Social norms and the fertility transition. Journal of Development Economics 80 (1), 1-38.

Myers, N.; Mittermeier, R. A.; Mittermeier, C. G.; da Fonseca, G. A.; \& Kent, J. (2000). Biodiversity hotspots for conservation priorities. Nature 403 (6772), 853-858.

Nantha, S. H.; \& Tisdell, C. (2009). The orangutan-oil palm conflict: Economic constraints and opportunities for conservation. Biodiversity and Conservation 18 (2), 487502.

Obidzinski, K.; Andriani, R.; Komarudin, H.; \& Andrianto, A. (2012). Environmental and Social Impacts of Oil Palm Plantations and their Implications for Biofuel Production in Indonesia. Ecology and Society 17 (1).

OECD-FAO (2015). OECD-FAO Agricultural Outlook 2015-2024. Paris: OECD Publishing. 
OECD-FAO (2016). OECD-FAO Agricultural Outlook 2016-2025. Paris: OECD Publishing.

Pepper, J. V. (2002). Robust inferences from random clustered samples: An application using data from the panel study of income dynamics. Economics Letters 75, 341-345.

Perfecto, I.; \& Vandermeer, J. (2010). The agroecological matrix as alternative to the land-sparing/agriculture intensification model. Proceedings of the National Academy of Sciences 107 (13), 5786-5791.

Phalan, B.; Balmford, A.; Green, R. E.; \& Scharlemann, J. P. W. (2011a). Minimising the harm to biodiversity of producing more food globally. Food Policy 36 (1), 62-71.

Phalan, B.; Onial, M.; Balmford, A.; \& Green, R. E. (2011b). Reconciling food production and biodiversity conservation: land sharing and land sparing compared. Science 333 (6047), 1289-1291.

Phelps, J.; Carrasco, L. R.; Webb, E. L.; Koh, L. P.; \& Pascual, U. (2013). Agricultural intensification escalates future conservation costs. Proceedings of the National Academy of Sciences 110 (19), 7601-7606.

Pingali, P. (2007). Westernization of Asian diets and the transformation of food systems. Implications for research and policy. Food Policy 32 (3), 281-298.

Qaim, M. (2017). Globalisation of agrifood systems and sustainable nutrition. The Proceedings of the Nutrition Society 76 (1), 12-21.

Quisumbing, M. A. R.; \& Otsuka, K. Land, trees, and women. Evolution of land tenure institutions in western Ghana and Sumatra. (Research report 121). Washington, DC: International Food Policy Research Institute (IFPRI).

Resosudarmo, I. A. P.; Atmadja, S.; Ekaputri, A. D.; Intarini, D. Y.; Indriatmoko, Y.; \& Astri, P. (2014). Does tenure security lead to REDD+ project effectiveness? Reflections from five emerging sites in Indonesia. World Development 55, 68-83.

Rindfuss, R. R.; \& Brewster, K. L. (1996). Childrearing and Fertility. Population and Development Review 22, 258-289.

Rist, L.; Feintrenie, L.; \& Levang, P. (2010). The livelihood impacts of oil palm: smallholders in Indonesia. Biodiversity and Conservation 19 (4), 1009-1024.

Robinson, B. E.; Holland, M. B.; \& Naughton-Treves, L. (2014). Does secure land tenure save forests? A meta-analysis of the relationship between land tenure and tropical deforestation. Global Environmental Change 29, 281-293.

Rosenzweig, M. R.; \& Evenson, R. (1977). Fertility, Schooling, and the Economic Contribution of Children of Rural India. An Econometric Analysis. Econometrica 45 (5), 1065-1079.

Rudel, T. K.; Schneider, L.; Uriarte, M.; Turner, B. L. 2.; Defries, R.; Lawrence, D. et al. (2009a). Agricultural intensification and changes in cultivated areas, 1970-2005. Proceedings of the National Academy of Sciences 106 (49), 20675-20680.

Rudel, T. K.; Defries, R.; Asner, G. P.; \& Laurance, W. F. (2009b). Changing drivers of deforestation and new opportunities for conservation. Conservation Biology 23 (6), 1396-1405. 
Schmitz, C.; van Meijl, H.; Kyle, P.; Nelson, G. C.; Fujimori, S.; Gurgel, A. et al. (2014). Land-use change trajectories up to 2050. Insights from a global agro-economic model comparison. Agricultural Economics 45 (1), 69-84.

Sibhatu, K. T.; Krishna, V. V.; \& Qaim, M. (2015). Production diversity and dietary diversity in smallholder farm households. Proceedings of the National Academy of Sciences of the United States of America 112 (34), 10657-10662.

Steffan-Dewenter, I.; Kessler, M.; Barkmann, J.; Bos, M. M.; Buchori, D.; Erasmi, S. et al. (2007). Tradeoffs between income, biodiversity, and ecosystem functioning during tropical rainforest conversion and agroforestry intensification. Proceedings of the National Academy of Sciences 104 (12), 4973-4978.

Stevenson, J. R.; Villoria, N.; Byerlee, D.; Kelley, T.; \& Maredia, M. (2013). Green Revolution research saved an estimated 18 to 27 million hectares from being brought into agricultural production. Proceedings of the National Academy of Sciences 110 (21), 83638368.

Stibig, H.-J.; Achard, F.; Carboni, S.; Raši, R.; \& Miettinen, J. (2014). Change in tropical forest cover of Southeast Asia from 1990 to 2010. Biogeosciences 11 (2), 247258.

Sukamdi; \& Mujahid, G. (2015). Internal Migration in Indonesia. (UNFPA Indonesia Monograph Series, 3). Jarkarta, Indonesia: UNFPA Indonesia.

Susanti, A.; \& Budidarsono, S. (2014). Land governance and oil palm development: Examples from Riau Province, Indonesia. In Zoomers, A.; \& Kaag, M. (Eds.): The great global land grab: Beyond the hype. London, UK: ZED Publisher, 119-134.

Tilman, D.; Cassman, K. G.; Matson, P. A.; Naylor, R.; \& Polasky, S. (2002). Agricultural sustainability and intensive production practices. Nature 418 (6898), 671-677.

Tscharntke, T.; Clough, Y.; Wanger, T. C.; Jackson, L.; Motzke, I.; Perfecto, I. et al. (2012). Global food security, biodiversity conservation and the future of agricultural intensification. Biological Conservation 151 (1), 53-59.

UNDP (2015). World Population Prospects: The 2015 Revision, Key Findings and Advance Tables. (Working Paper No. ESA/P/WP.241.). New York, USA.: United Nations.

US Department of Agriculture (2017). Oil crops yearbook. Available online at https://www.ers.usda.gov/data-products/oil-crops-yearbook.aspx, checked on 09.02.2018.

Valin, H.; Sands, R. D.; van der Mensbrugghe, D.; Nelson, G. C.; Ahammad, H.; Blanc, E. et al. (2014). The future of food demand. Understanding differences in global economic models. Agricultural Economics 45 (1), 51-67.

van Garderen, K. J.; \& Shah, C. (2002). Exact interpretation of dummy variables in semilogarithmic equations. The Econometrics Journal 5 (1), 149-159.

van Vliet, J.; Groot, H. L.F. de; Rietveld, P.; \& Verburg, P. H. (2015). Manifestations and underlying drivers of agricultural land use change in Europe. Landscape and Urban Planning 133, 24-36.

Verheye, W. (2010). Growth and Production of Oil Palm. In Verheye, W. (Ed.): Land Use, Land Cover and Soil Sciences. Oxford, UK: Encyclopedia of Life Support Systems (EOLSS), UNE SCO-EOLSS. 
Vijay, V.; Pimm, S. L.; Jenkins, C. N.; \& Smith, S. J. (2016). The Impacts of Oil Palm on Recent Deforestation and Biodiversity Loss. PloS one 11 (7), e0159668.

Wanamaker, M. H. (2012). Industrialization and Fertility in the Nineteenth Century. Evidence from South Carolina. The Journal of Economic History 72 (1), 168-196.

Wicke, B.; Sikkema, R.; Dornburg, V.; \& Faaij, A. (2011). Exploring land use changes and the role of palm oil production in Indonesia and Malaysia. Land Use Policy 28 (1), 193-206.

Wilcove, D. S.; \& Koh, L. P. (2010). Addressing the threats to biodiversity from oilpalm agriculture. Biodiversity and Conservation 19 (4), 999-1007.

Wilcove, D. S.; Giam, X.; Edwards, D. P.; Fisher, B.; \& Koh, L. P. (2013). Navjot's nightmare revisited: Logging, agriculture, and biodiversity in Southeast Asia. Trends in ecology \& evolution 28 (9), 531-540.

Woittiez, L. S.; van Wijk, M. T.; Slingerland, M.; van Noordwijk, M.; \& Giller, K. E. (2017). Yield gaps in oil palm: A quantitative review of contributing factors. European Journal of Agronomy 83, 57-77.

Wollni, M.; \& Andersson, C. (2014). Spatial patterns of organic agriculture adoption. Evidence from Honduras. Ecological Economics 97, 120-128.

Wooldridge, J. M. (2002). Econometric analysis of cross section and panel data. Cambridge, Massachusetts: MIT Press.

World Bank (2016). Official exchange rate (LCU per US\$, period average). Washington DC, USA. Available online at http://data.worldbank.org/indicator/PA.NUS.FCRF, checked on 23.07.2016.

World Bank (2018a). Indonesia Database for Policy and Economic Research (INDODAPOER). Available online at https://data.worldbank.org/data-catalog/indonesiadatabase-for-policy-and-economic-research, checked on 25.10.2017.

World Bank (2018b). Official exchange rate (LCU per US\$, period average). Available online at https://data.worldbank.org/indicator/PA.NUS.FCRF?locations=ID\&view=chart, checked on 06.03.2018.

World Bank (2018c). World Development Indicators. Available online at http://databank.worldbank.org/data/reports.aspx?source=world-development-indicators, checked on 05.03.2018.

York, R.; Rosa, E. A.; \& Dietz, T. (2003). Footprints on the Earth. The Environmental Consequences of Modernity. American Sociological Review 68 (2), 279-300. 
General appendix 


\section{Farm household survey questionnaire}

(Farm survey; round 2; 2015)

1. Household identification

\begin{tabular}{|c|c|c|c|}
\hline 1. Village (name): & & & \\
\hline 2. Dusun (name or number): & & & \\
\hline 3. RT (number): & & & \\
\hline 4. Household code (given by supervisor): & & & \\
\hline 5. Name of the current household head: & & & \\
\hline 6. Name of the current respondent: & & & \\
\hline $\begin{array}{l}\text { 7. Was the household interviewed in 2012? (If } \\
\text { no, go to question 14) }\end{array}$ & & Yes/No & \\
\hline 8. Name of household head in 2012: & & & \\
\hline 9. Did the household head change since 2012? & & $\mathrm{Yes} / \mathrm{No}$ & \\
\hline 10. Name of respondent in 2012: & & & \\
\hline 11. Did the respondent change since 2012? & & Yes/No & \\
\hline 12. Why did the respondent change? (Code A) & & & \\
\hline $\begin{array}{l}\text { 13. Did the household change place of } \\
\text { residence after 2012? }\end{array}$ & & Yes/No & \\
\hline 14. GPS co-ordinates of the household: & ............... & $\mathrm{S} ; \ldots \ldots \ldots \ldots \ldots \ldots \mathrm{E} ; \ldots \ldots$ & ......Alt \\
\hline 15. Mobile phone numbers: & $\begin{array}{l}\text { Primary: } \\
\text { Secondary: } \\
\text { Tertiary: }\end{array}$ & & \\
\hline $\begin{array}{l}\text { 16. Distance from the household's dwelling to } \\
\text { the nearest market / trading center }(\mathrm{km})\end{array}$ & & & \\
\hline $\begin{array}{l}\text { 17. Household interviewed by other sub- } \\
\text { projects? }\end{array}$ & C01: Yes / No & B09: Yes / $\mathrm{Y}$ & \\
\hline 18. Interviewer (name): & & & \\
\hline 19. Supervisor (name): & & & \\
\hline 20. Date of interview: & ...../ ...../ 2015 & Enumerator's signature: & \\
\hline $\begin{array}{l}\text { 21. Date questionnaire was checked by } \\
\text { supervisor: }\end{array}$ & ...../ ...../ 2015 & Supervisor's signature: & \\
\hline
\end{tabular}

Code A: currently out of village $=1$; moved out of the household $=2$; passed away $=3$; others $($ specify $)=4$. 
2. General farm data

2.1 Cropping activities

What kind of crops are you currently growing on your farm:

\begin{tabular}{|c|c|c|c|c|}
\hline & \multicolumn{2}{|c|}{ Area under cultivation (ha) } & \multicolumn{2}{|c|}{$\begin{array}{l}\text { For how much of this land (ha), you } \\
\text { have }\end{array}$} \\
\hline & In 2012 & In 2015 & $\begin{array}{l}\text { Systematic } \\
\text { certificate }\end{array}$ & $\begin{array}{l}\text { Sporadic } \\
\text { certificate }\end{array}$ \\
\hline \multicolumn{5}{|l|}{ 1. Oil palm (total) } \\
\hline \multicolumn{5}{|l|}{ a. Oil palm (independent) } \\
\hline \multicolumn{5}{|l|}{ b. Oil palm (under contract) } \\
\hline \multicolumn{5}{|l|}{ 2. $\quad$ Plantation and jungle rubber (total) } \\
\hline \multicolumn{5}{|l|}{$\begin{array}{l}\text { a. Plantation and jungle rubber } \\
\text { (independent) }\end{array}$} \\
\hline \multicolumn{5}{|l|}{$\begin{array}{l}\text { b. Plantation and jungle rubber } \\
\text { (under contract) }\end{array}$} \\
\hline \multicolumn{5}{|l|}{ 3. Other plantation crops 1: } \\
\hline \multicolumn{5}{|l|}{ 4. Other plantation crops 2: } \\
\hline \multicolumn{5}{|l|}{ 5. Other plantation crops 3: } \\
\hline \multicolumn{5}{|l|}{ 6. Homestead and kitchen garden } \\
\hline \multicolumn{5}{|l|}{ 7. Rice } \\
\hline \multicolumn{5}{|l|}{ 8. Other annual crop 1: } \\
\hline \multicolumn{5}{|l|}{ 9. Other annual crop 2: } \\
\hline \multicolumn{5}{|l|}{ 10. Other annual crop 3: } \\
\hline $\begin{array}{l}\text { 11. Fallow land (no cultivation in last } 12 \\
\text { months) }\end{array}$ & & & & \\
\hline
\end{tabular}

2.2 Land ownership and management

\begin{tabular}{|c|c|c|}
\hline \multirow{2}{*}{$\begin{array}{l}\text { 1a. In the last } 12 \text { months, did you own any } \\
\text { land, which is cultivated by some other } \\
\text { household? (e.g., sharecropping as landlord) }\end{array}$} & \multirow{3}{*}{ Yes/No } & If yes, under output sharing?.......... Yes/No \\
\hline & & $\begin{array}{l}\text { If yes, share of harvest received as rent:...........\% } \\
\text { Size of land under output sharing:......... ha }\end{array}$ \\
\hline (Land should be included in table 2.1) & & $\begin{array}{l}\text { If no, rent you received for renting out:..........Rp ' } 000 / \text { ha } / \text { year } \\
\text { Size of land under rent arrangements:....... ha }\end{array}$ \\
\hline \multirow{3}{*}{$\begin{array}{l}\text { 1b. In the last } 12 \text { months, did you own any } \\
\text { land, which is cultivated by a company? }\end{array}$} & \multirow{3}{*}{ Yes/No } & If yes: \\
\hline & & Size of such land: .......... ha \\
\hline & & Rent you received for renting out:.......... '000/ha/year. \\
\hline \multirow{4}{*}{$\begin{array}{l}\text { 2. In the last } 12 \text { months, did you cultivate } \\
\text { any land together with another farmer or } \\
\text { group of farmers or co-operative society? } \\
\text { (Collective farming) }\end{array}$} & \multirow{4}{*}{ Yes/No } & If yes: \\
\hline & & Total land under this arrangement:........ ha \\
\hline & & How much of the land you own is under this arrangement? ..... ha \\
\hline & & No. of farmers in the group: ........... \\
\hline
\end{tabular}




\begin{tabular}{|c|c|c|}
\hline \multirow{3}{*}{$\begin{array}{l}\text { 3a. In the last } 12 \text { months, did you cultivate } \\
\text { any land, owned by others? (e.g., sharecropping } \\
\text { as tenant) }\end{array}$} & \multirow{3}{*}{ Yes/No } & If yes, under output sharing?.......... Yes/No \\
\hline & & $\begin{array}{l}\text { If yes, share of harvest received as wage:..........\% } \\
\text { Size of land under output sharing:......... ha }\end{array}$ \\
\hline & & $\begin{array}{l}\text { If no, rent you paid for renting in:..............Rp ' } 000 / \text { ha/year. } \\
\text { Size of land under rent arrangement:........ ha }\end{array}$ \\
\hline \multirow{3}{*}{$\begin{array}{l}\text { 3b. In } 2012 \text {, did you cultivate any land, } \\
\text { owned by others? (e g. sharecropping as laborer) }\end{array}$} & \multirow[t]{3}{*}{ Yes/No } & If yes, under output sharing?.......... Yes/No \\
\hline & & $\begin{array}{l}\text { If yes, share of harvest received as wage:............\% } \\
\text { Size of land under output sharing:......... ha }\end{array}$ \\
\hline & & $\begin{array}{l}\text { If no, rent you paid for renting in:............. Rp '000/ha/year } \\
\text { Size of land under rent arrangements:........ ha }\end{array}$ \\
\hline
\end{tabular}

\section{History of crop cultivation}

\subsection{Migration (ONLY FOR NEWLY ADDED FARMERS, OTHERWISE CONTINUE}

\section{TO 3.2)}

a. Did the household migrate from somewhere to this village? ....... (Yes/No) (If no, go to 3.2).

b. If yes, did the household migrate as part of transmigrant program? ............ (Yes/No)

c. If yes, the crop associated with transmigrant program: Oil palm/ Rubber/Others (specify):........

Details of starting of cultivation and contract for transmigrant households:

\begin{tabular}{|l|l|}
\hline 1. When did the household migrate to the village? (Year) & \\
\hline 2. Who was the head of the household at the time of migration? (Code A) & \\
\hline $\begin{array}{l}\text { 3. If you were not the head of household at time of migration, age of the } \\
\text { household head at that time (Years) }\end{array}$ & \\
\hline 4. The place from where the household migrated to this village? (Code B) & \\
\hline $\begin{array}{l}\text { 5. What was the major source of income for the household before migration? } \\
\text { (Code C) }\end{array}$ & \\
\hline $\begin{array}{l}\text { 6. What was your household size before migration? (number of household } \\
\text { members) }\end{array}$ & \\
\hline 7. How many of your family members.... (number) & Yes / No \\
\hline $\begin{array}{l}\text { a. Came to this village in your group of migration? (including respondent) } \\
\text { b. Arrived in this village after you came? (exclude the members born here) }\end{array}$ & \\
\hline 8. Was there a house already built for you in this village (e.g. by the government)? & a. Plantation (ha) \\
\hline $\begin{array}{l}\text { 9. What was the size of land provided by } \\
\text { government as part of the transmigrant } \\
\text { program? }\end{array}$ & b. Food crops (ha) \\
\hline $\begin{array}{l}\text { 10. Number of years you obtained livelihood assistance (food, cloths etc.) from } \\
\text { government? }\end{array}$ & \\
\hline
\end{tabular}

Code A: current $\mathrm{HH}$ head = 1, father/mother of current $\mathrm{HH}$ head = 2; grandparent of current $\mathrm{HH}$ head $=3$; brother $/$ sister of current $\mathrm{HH}$ head $=4$; other (specify $)=5$

Code B: Other part of Jambi $=1$; Java $=2$; North Sumatra $=3$; South Sumatra $=4$; Kalimantan $=$ 5; Sulawesi $=6$; others (specify) $=7$

Code C: crops $=1$; fisheries and livestock $=2$; wage labour $=3$; small business $=4$; others (specify) $=5$ 
3.2. Household details at plantation start (Do not include the crop covered under transmigrant program. But if a transmigrant household started another crop later, that information should be included in this table.)

\begin{tabular}{|c|c|c|}
\hline & Oil palm & $\begin{array}{c}\text { Plantation } \\
\text { or jungle } \\
\text { rubber }\end{array}$ \\
\hline 1. Have you ever cultivated the crop? (If no, go to next column) & Yes / No & Yes / No \\
\hline $\begin{array}{l}\text { a. Was the household already interviewed in 2012? (If no, go to } \\
\text { question 2) }\end{array}$ & Yes / No & Yes / No \\
\hline $\begin{array}{l}\text { b. Have you started cultivating the crop after 2012? (If no, go to } \\
\text { next column) }\end{array}$ & Yes / No & Yes / No \\
\hline 2. When did the household start cultivating the crop? (Year) & & \\
\hline $\begin{array}{l}\text { 3. Which of your family members first started the cultivation/ } \\
\text { obtained the plantation? (Code A) (If Code } A=1 \text { go to question 5) }\end{array}$ & & \\
\hline $\begin{array}{l}\text { 4. If some other household member (and not the current head) } \\
\text { started the plantation, then: }\end{array}$ & & \\
\hline $\begin{array}{l}\text { a. Relation of that member with the current household head } \\
\text { (Code B) }\end{array}$ & & \\
\hline b. Age of this member at starting of the estate (Years) & & \\
\hline c. Gender of this household member $($ male $=0$, female $=1$ ) & & \\
\hline $\begin{array}{l}\text { d. Education of this member when the estate was started (Years in } \\
\text { school): }\end{array}$ & & \\
\hline e. Was he/she residing in this village for all his/her life? & Yes / No & Yes / No \\
\hline f. If no, when did he/she migrate to this village (Year) & & \\
\hline $\begin{array}{l}\text { 5. Was the whole estate planted by the household? (Ifyes, go to } \\
\text { question } 6 \text { ) }\end{array}$ & Yes / No & Yes / No \\
\hline a. Size of the estate that was not established by household (ha) & & \\
\hline b. Number of oil palm / rubber trees already existing in that field & & \\
\hline c. What was the average age of trees? (years) & & \\
\hline 6. Before the plantation was started, & & \\
\hline $\begin{array}{l}\text { a. How many adult family members were there in your household } \\
\text { (number)? }\end{array}$ & & \\
\hline $\begin{array}{l}\text { b. How much land did your household have under cultivation? } \\
\text { (ha) }\end{array}$ & & \\
\hline $\begin{array}{l}\text { c. For how much of this land did your household have a land } \\
\text { title? (ha) }\end{array}$ & & \\
\hline d. Were any of your relatives already cultivating the crop? & Yes / No & Yes / No \\
\hline $\begin{array}{l}\text { e. How many of the other farmer households in your } \\
\text { neighborhood/RT started the cultivation before you? (number) }\end{array}$ & & \\
\hline $\begin{array}{l}\text { 7. Total number of households in the neighborhood/ RT at that } \\
\text { point of time? }\end{array}$ & & \\
\hline & $\begin{array}{l}\text { Please go to } \\
\text { next column }\end{array}$ & $\begin{array}{l}\text { Please go to } \\
\text { next table }\end{array}$ \\
\hline
\end{tabular}

Code A: current $\mathrm{HH}$ head = 1; previous $\mathrm{HH}$ head = 2; acquired through marriage $=3$; others (specify) $=4$

Code B: father $/$ mother $=1$; grandparent $=2 ;$ brother $=3$; in-laws $=4$; others $($ specify $)=5$ 


\subsection{Oil palm}

- If the farmer ever cultivated oil palm (If not, go to 3.4.):

a. Area under oil palm in 2012:........... ha

b. How many hectares do you have today? ha

- If newly added farmer:

c. With how many hectares did you start cultivation? ..ha

d. How many times was the area under this crop changed from the start of cultivation until today? ......

\section{- If the farmer was already interviewed in 2012:}

e. How many times the area under the crop was changed after 2012 until today? times (if

0 , go to 3.4 )

Details of changes (changes after 2012 for farmers interviewed in 2012; all changes for newly added farmers):

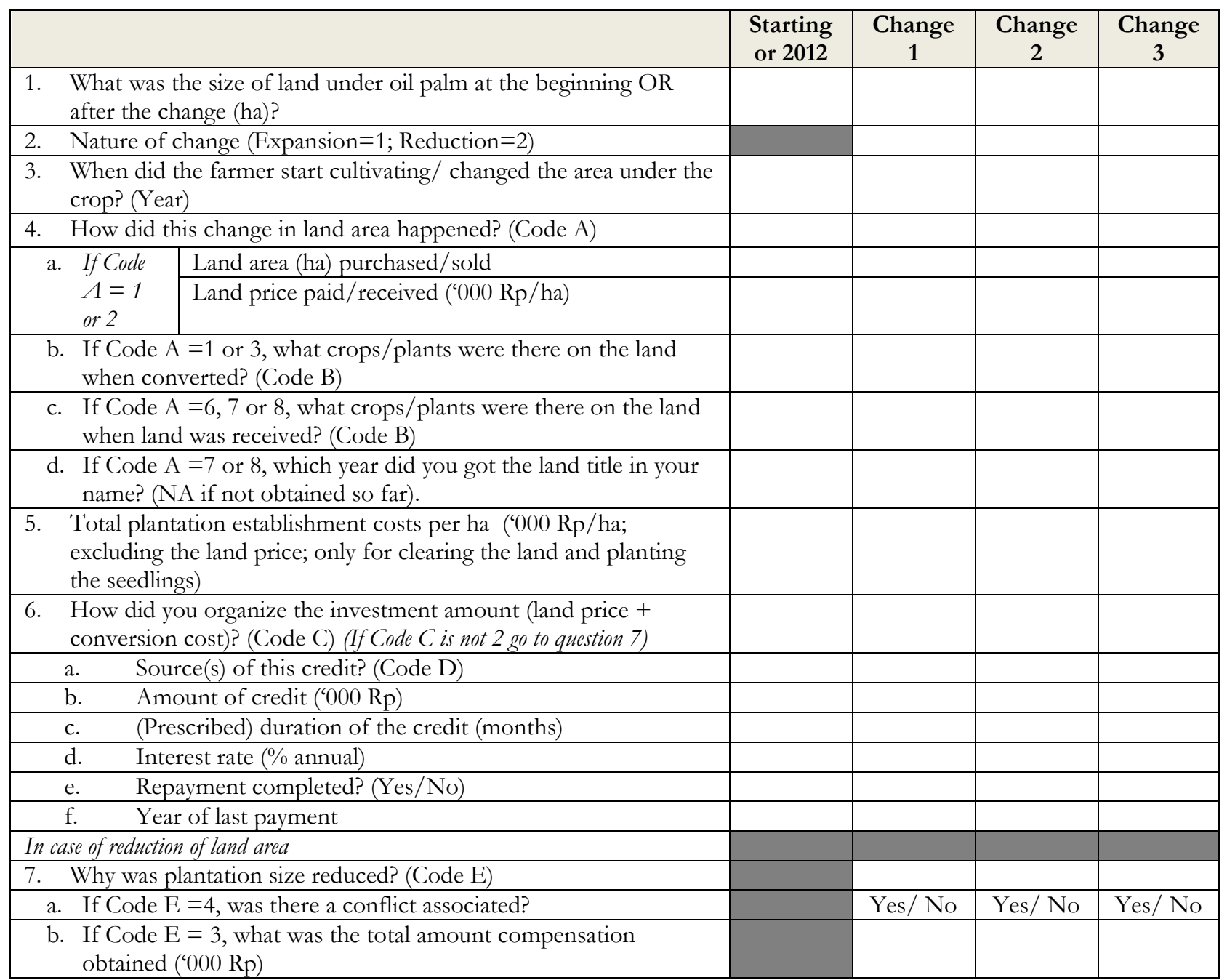

Code A: purchasing = 1; selling $=2$; converted from $/$ to other crops $=3$; conversion from forest $=4$; obtained as part of a government program (e.g. “transmigransi”) $=5$; established plantation obtained from company $=6$; inherited $=7$; received as gift $=8$; others $($ specify $)=9$

Code B: oil palm = 1; plantation rubber $=2$; jungle rubber $=3$; other plantation $=4$; annual crops $($ specify $)=5$; grassland $=6$; forest $=7$; bush $=8$; others (specify) $=9$

Code C: savings $=1$; credit $=2$; parents $/$ spouse $=3$; no need to pay at the beginning $=4$; others $($ specify $)=5$

Code D: banks $=1$; private company $=2$; money lender $=3$; friends $/$ relatives $=4$; farmer cooperative $=5$; other farmers $=6$; others (specify) $=7$

Code E: land sold=1; land contracted out to other family = 2; land submitted to a company $=3$; land lost without compensation $=4$; land given away to other family member or relative $=5$; other $($ specify $)=6$ 


\subsection{Plantation and Jungle Rubber}

- If the farmer ever cultivated rubber (If not, go to 3.5.):

a. Area under rubber in 2012:........... ha

b. How many hectares do you have today? ha

- If newly added farmer:

c. With how many hectares did you start cultivation? ..ha

d. How many times was the area under this crop changed from the start of cultivation until today? ......

- If the farmer was already interviewed in 2012:

e. How many times the area under the crop was changed after 2012 until today? times (if

0 , go to 3.5$)$

Details of changes (changes after 2012 for farmers interviewed in 2012; all changes for newly added farmers):

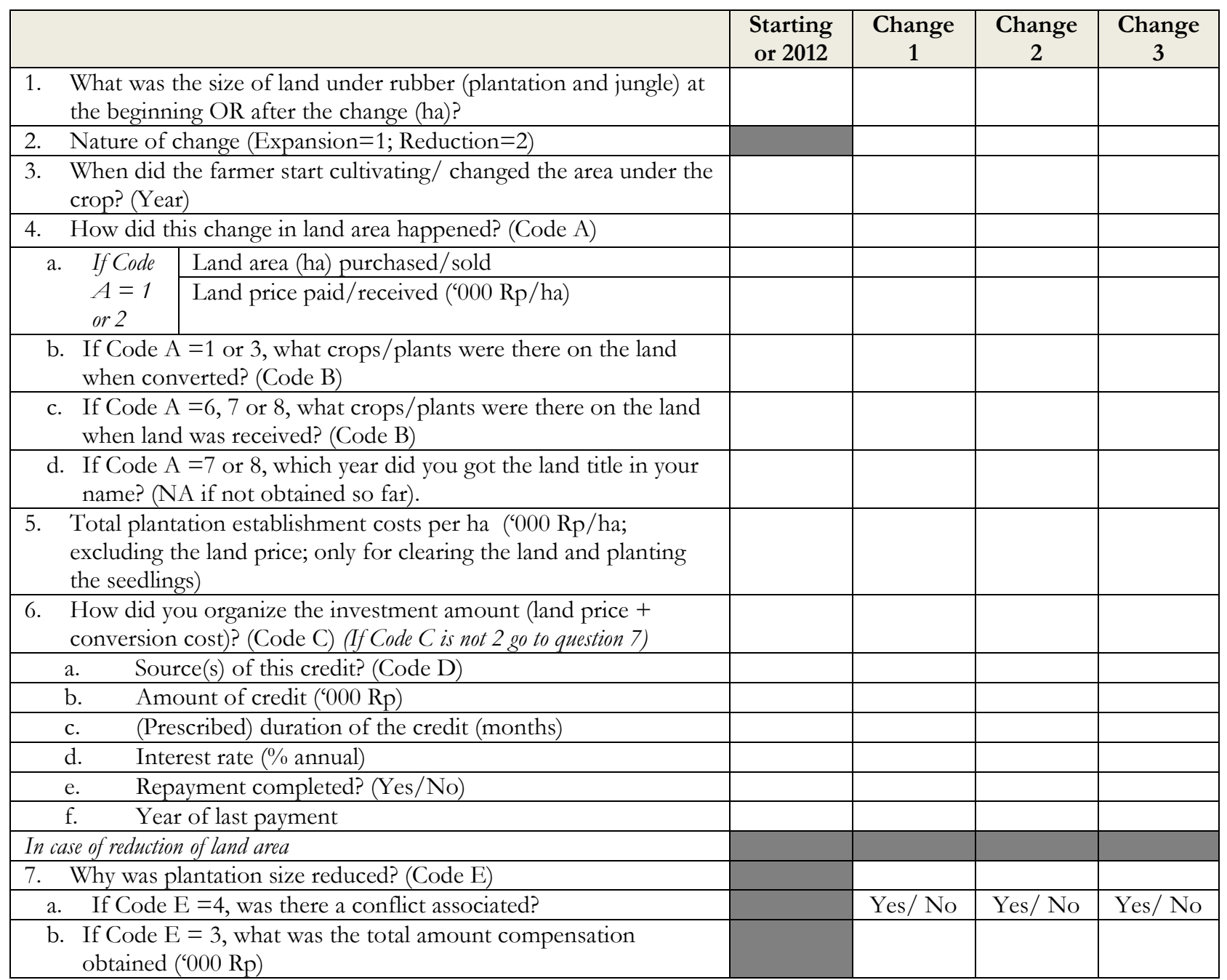

Code A: purchasing = 1; selling $=2$; converted from $/$ to other crops $=3$; conversion from forest $=4$; obtained as part of a government programme (e.g."transmigransi") $=5$; established plantation obtained from company $=6$; inherited $=$ 7 ; received as gift $=8$; others (specify) $=9$

Code B: oil palm = 1; plantation rubber $=2$; jungle rubber $=3$; other plantation $=4$; annual crops $($ specify $)=5$; grassland $=6$; forest $=7$; bush $=8$; others (specify) $=9$

Code C: savings $=1$; credit $=2$; parents $/$ spouse $=3$; no need to pay at the beginning $=4$; others $($ specify $)=5$

Code D: banks $=1$; private company $=2$; money lender $=3$; friends $/$ relatives $=4$; farmer cooperative $=5$; other farmers $=6$; others (specify) $=7$

Code E: land sold=1; land contracted out to other family = 2; land submitted to a company = 3; land lost without compensation $=4$; land given away to other family member or relative $=5$; other (specify) $=6$ 


\subsection{Changes in area under other crops (from the establishment of household)}

a. Kitchen garden

Have you ever maintained a kitchen garden? ............... (Yes/No). (If no, go to next table)

\begin{tabular}{|c|c|c|c|c|}
\hline & Starting & Change 1 & $\begin{array}{l}\text { Change } \\
2\end{array}$ & $\begin{array}{c}\text { Change } \\
3\end{array}$ \\
\hline $\begin{array}{l}\text { 1. What was the size of land under these crops at the beginning OR } \\
\text { after the change (ha)? }\end{array}$ & & & & \\
\hline 2. Nature of change (Expansion=1; Reduction $=2$ ) & & & & \\
\hline $\begin{array}{l}\text { 3. When did the farmer start cultivating/ changed the area under the } \\
\text { crop? (Year) }\end{array}$ & & & & \\
\hline 4. How did this change in land area happened? (Code A) & & & & \\
\hline $\begin{array}{l}\text { a. If Code } \mathrm{A}=1 \text { or } 3 \text {, what crops/plants were there on the } \\
\text { land when converted? (Code B) }\end{array}$ & & & & \\
\hline $\begin{array}{l}\text { b. If Code } \mathrm{A}=6,7 \text { or } 8 \text {, what crops/plants were there on } \\
\text { the land when land was received? (Code B) }\end{array}$ & & & & \\
\hline
\end{tabular}

How many $\%$ of the area are productive now :.............

How many $\%$ of the area were productive three years ago :..............

How many $\%$ of the area were productive ten years ago :....

b. Homestead garden

Have you ever maintained a homestead garden? ............... (Yes/No). (If no, go to next table)

\begin{tabular}{|c|c|c|c|c|}
\hline & Starting & $\begin{array}{c}\text { Change } \\
1\end{array}$ & $\begin{array}{l}\text { Change } \\
2\end{array}$ & $\begin{array}{c}\text { Change } \\
3 \\
\end{array}$ \\
\hline $\begin{array}{l}\text { 1. What was the size of land under these crops at the beginning OR } \\
\text { after the change (ha)? }\end{array}$ & & & & \\
\hline 2. Nature of change (Expansion=1; Reduction $=2$ ) & & & & \\
\hline $\begin{array}{l}\text { 3. When did the farmer start cultivating/ changed the area under the } \\
\text { crop? (Year) }\end{array}$ & & & & \\
\hline 4. How did this change in land area happened? (Code A) & & & & \\
\hline $\begin{array}{l}\text { a. If Code } \mathrm{A}=1 \text { or } 3 \text {, what crops/plants were there on the } \\
\text { land when converted? (Code B) }\end{array}$ & & & & \\
\hline $\begin{array}{l}\text { b. If Code } A=6,7 \text { or } 8 \text {, what crops/plants were there on the } \\
\text { land when land was received? (Code B) }\end{array}$ & & & & \\
\hline
\end{tabular}

How many $\%$ of the area are productive now :..............

How many $\%$ of the area were productive three years ago :............

How many $\%$ of the area were productive ten years ago :...

c. Other annual and perennial crops

Have you ever maintained other annual and perennial crops? .............. (Yes/No). (If no, go to next table)

\begin{tabular}{|c|c|c|c|c|}
\hline & Starting & $\begin{array}{c}\text { Change } \\
1\end{array}$ & $\begin{array}{c}\text { Change } \\
2\end{array}$ & $\begin{array}{c}\text { Change } \\
3\end{array}$ \\
\hline $\begin{array}{l}\text { 1. What was the size of land under the crop at the beginning OR after } \\
\text { the change (ha)? }\end{array}$ & & & & \\
\hline 2. Nature of change (Expansion=1; Reduction=2) & & & & \\
\hline $\begin{array}{l}\text { 3. When did the farmer start cultivating/ changed the area under the } \\
\text { crop? (Year) }\end{array}$ & & & & \\
\hline 4. How did this change in land area happened? (Code A) & & & & \\
\hline $\begin{array}{l}\text { a. If Code } A=1 \text { or } 3 \text {, what crops/plants were there on the } \\
\text { land when converted? (Code B) }\end{array}$ & & & & \\
\hline $\begin{array}{l}\text { b. If Code } A=6,7 \text { or } 8 \text {, what crops/plants were there on } \\
\text { the land when land was received? (Code B) }\end{array}$ & & & & \\
\hline
\end{tabular}

How many $\%$ of the area are productive now :.............

How many $\%$ of the area were productive three years ago :.............

How many $\%$ of the area were productive ten years ago :

Code A: purchasing = 1; selling $=2$; converted from $/$ to other crops $=3$; conversion from forest $=4$; obtained as part of a government programme (e.g. "transmigransi") $=5$; established plantation obtained from company $=6$; inherited $=$ 7 ; received as gift $=8$; others (specify) $=9$

Code B: oil palm $=1$; plantation rubber $=2$; jungle rubber $=3$; other plantation $=4$; annual crops $($ specify $)=5$; grassland $=6$; forest $=7$; bush $=8$; others ( pecify) $=9$ 
3.6 Information and institutional context of smallholder plantation (Complete the columns irrespective of whether the farmer cultivates this crop)

\begin{tabular}{|c|c|c|}
\hline & Oil palm & Plantation \& Jungle Rubber \\
\hline $\begin{array}{l}\text { 1. Have you or any of the household members ever associated with the crop as a trader } \\
\text { or laborer? }\end{array}$ & $\begin{array}{c}\text { Yes/ No } \\
\text { (If no, go to next column) }\end{array}$ & $\begin{array}{l}\text { Yes / No } \\
\text { (If no, go to next table) }\end{array}$ \\
\hline a. If yes, how or in what context? & Trader / Laborer / Both & Trader / Laborer / Both \\
\hline If involved as a trader: & & \\
\hline $\begin{array}{l}\text { b. In which year did you/ household member start associating with the crop as a } \\
\text { trader? }\end{array}$ & & \\
\hline $\begin{array}{l}\text { c. In which year did you/ household member stop associating with the crop as a } \\
\text { trader? } \\
\text { (Put NA if still working as trader) }\end{array}$ & & \\
\hline If involved as a laborer: & & \\
\hline $\begin{array}{l}\text { d. Were you/household member associated as a laborer/tenant in a sharecropping or } \\
\text { a wage arrangement? }\end{array}$ & Sharecropping / Wage / Both & Sharecropping / Wage / Both \\
\hline If involved as a wage laborer: & & \\
\hline $\begin{array}{l}\text { e. In which year did you/household member start associating with the crop as a wage } \\
\text { laborer? }\end{array}$ & & \\
\hline $\begin{array}{l}\text { f. In which year did you/household member stop associating with the crop as a wage } \\
\text { laborer? } \\
\text { (Put NA if still working as laborer) }\end{array}$ & & \\
\hline If involved as a sharecropping tenant: & & \\
\hline $\begin{array}{l}\text { g. In which year did you/household member start associating with the crop as a } \\
\text { tenant? }\end{array}$ & & \\
\hline $\begin{array}{l}\text { h. In which year did you/household member stop associating with the crop as a } \\
\text { tenant? } \\
\text { (Put NA if still working as laborer) }\end{array}$ & & \\
\hline $\begin{array}{l}\text { 2. In } 1992 \text { or at the time of household establishment (whichever is recent) did any of } \\
\text { your close relatives cultivate the crop? }\end{array}$ & Yes/No & Yes/No \\
\hline
\end{tabular}


HH number: ..........................................

4. Cost of cultivation of all crops cultivated during the last 12 months (including the kitchen garden)

a. How many permanent laborers are employed on your farm? (number)

b. Wages paid per month: ............... ('000 Rp/month)

\begin{tabular}{|c|c|c|c|c|c|c|c|c|c|c|c|}
\hline \multirow{2}{*}{\multicolumn{2}{|c|}{ Crop name }} & \multicolumn{5}{|c|}{ Perennials } & \multicolumn{4}{|c|}{ Annuals } & \multirow{2}{*}{$\begin{array}{l}\text { Home- } \\
\text { stead } \\
\text { and } \\
\text { kitchen } \\
\text { garden }\end{array}$} \\
\hline & & Oil palm & $\begin{array}{l}\text { Rubber } \\
\text { (plantation } \\
+ \text { jungle) }\end{array}$ & $\begin{array}{l}\text { Other } \\
1\end{array}$ & $\begin{array}{l}\text { Other } \\
2\end{array}$ & $\begin{array}{l}\text { Other } \\
3\end{array}$ & $\begin{array}{l}\text { Crop } \\
\quad 1\end{array}$ & $\begin{array}{l}\text { Crop } \\
2\end{array}$ & $\begin{array}{l}\text { Crop } \\
3\end{array}$ & $\begin{array}{c}\text { Crop } \\
4\end{array}$ & \\
\hline \multicolumn{2}{|l|}{$\begin{array}{l}\text { 1. Name of the main crop (if an annual crop is cultivated in more } \\
\text { than one season, consider it as an additional crop) }\end{array}$} & & & & & & & & & & \\
\hline \multicolumn{2}{|l|}{$\begin{array}{l}\text { 2. Which household members are more involved in crop } \\
\text { management, like selecting varieties, choosing fertilizers etc. } \\
\text { (Male }=1 ; \text { Female }=2 ; \text { Both equally }=3 \text {.) }\end{array}$} & & & & & & & & & & \\
\hline \multicolumn{2}{|l|}{ 3. Total area under cultivation under this crop (ha) } & & & & & & & & & & \\
\hline \multicolumn{2}{|l|}{ 4. Area under production (ha) } & & & & & & & & & & \\
\hline \multicolumn{2}{|l|}{ 5. Area under share-cropping (ha) } & & & & & & & & & & \\
\hline \multicolumn{2}{|l|}{ 6. If yes, which share does the farmer receive? } & & & & & & & & & & \\
\hline \multicolumn{2}{|l|}{ 7. Do you intercrop the plots? (If no, go to question 13) } & $\begin{array}{l}\text { Yes/ } \\
\text { No }\end{array}$ & $\begin{array}{l}\text { Yes/ } \\
\text { No }\end{array}$ & $\begin{array}{l}\text { Yes/ } \\
\text { No }\end{array}$ & $\begin{array}{l}\text { Yes/ } \\
\text { No }\end{array}$ & $\begin{array}{l}\text { Yes/ } \\
\text { No }\end{array}$ & $\begin{array}{l}\text { Yes/ } \\
\text { No }\end{array}$ & $\begin{array}{l}\text { Yes/ } \\
\text { No }\end{array}$ & $\begin{array}{l}\text { Yes/ } \\
\text { No }\end{array}$ & $\begin{array}{l}\text { Yes/ } \\
\text { No }\end{array}$ & \\
\hline \multicolumn{2}{|l|}{$\begin{array}{l}\text { 8. If yes, number of intercrops (report number of types of crops in } \\
\text { homestead and kitchen farm) }\end{array}$} & & & & & & & & & & \\
\hline \multicolumn{2}{|l|}{ 9. Area under intercropping (ha) } & & & & & & & & & & \\
\hline \multirow{3}{*}{$\begin{array}{l}\text { 10. Names of major intercrops (different plants/trees in case of } \\
\text { homestead farming) }\end{array}$} & 1 & & & & & & & & & & \\
\hline & 2 & & & & & & & & & & \\
\hline & 3 & & & & & & & & & & \\
\hline \multicolumn{12}{|l|}{ 11. Intercrop 1} \\
\hline \multicolumn{12}{|l|}{ a. Number of harvests during last 12 months } \\
\hline \multicolumn{12}{|l|}{ b. Quantity $(\mathrm{kg})$ produced during last 12 months } \\
\hline \multicolumn{12}{|l|}{ c. Quantity (kg) marketed } \\
\hline \multicolumn{12}{|l|}{ d. Avg. price received during last 12 months ('000 Rp/kg) } \\
\hline \multicolumn{12}{|l|}{ 12. Intercrop 2} \\
\hline a. Number of harvests during last 12 months & & & & & & & & & & & \\
\hline b. Quantity (kg) produced during last 12 months & & & & & & & & & & & \\
\hline c. Quantity (kg) marketed & & & & & & & & & & & \\
\hline d. Avg. price received during last 12 months ('000 Rp/kg) & & & & & & & & & & & \\
\hline
\end{tabular}


HH number: ..................................

Section 1

\begin{tabular}{|c|c|c|c|c|c|c|c|c|c|c|}
\hline \multirow[t]{2}{*}{ Crop name } & \multicolumn{5}{|c|}{ Perennials } & \multicolumn{4}{|c|}{ Annuals } & \multirow{2}{*}{$\begin{array}{l}\text { Home- } \\
\text { stead } \\
\text { and } \\
\text { Kitchen } \\
\text { garden }\end{array}$} \\
\hline & Oil palm & $\begin{array}{l}\text { Rubber } \\
\text { (plantation } \\
+ \text { jungle) }\end{array}$ & $\begin{array}{l}\text { Other } \\
1\end{array}$ & $\begin{array}{l}\text { Other } \\
2\end{array}$ & $\begin{array}{c}\text { Other } \\
3\end{array}$ & $\begin{array}{c}\text { Crop } \\
1\end{array}$ & $\begin{array}{l}\text { Crop } \\
2\end{array}$ & $\begin{array}{c}\text { Crop } \\
3\end{array}$ & $\begin{array}{c}\text { Crop } \\
4\end{array}$ & \\
\hline \multicolumn{11}{|l|}{ 13. Intercrop 3} \\
\hline a. Number of harvests during last 12 months & & & & & & & & & & \\
\hline b. Quantity (kg) produced during last 12 months & & & & & & & & & & \\
\hline c. Quantity (kg) marketed & & & & & & & & & & \\
\hline d. Avg. price received during last 12 months ('000 Rp/kg) & & & & & & & & & & \\
\hline \multicolumn{11}{|l|}{ 14. Main Crop } \\
\hline a. Number of harvests during last 12 months & & & & & & & & & & \\
\hline b. Quantity (kg) produced during last 12 months & & & & & & & & & & \\
\hline c. Quantity (kg) marketed & & & & & & & & & & \\
\hline d. Avg. price received during last 12 months ('000 Rp/kg) & & & & & & & & & & \\
\hline \multicolumn{11}{|l|}{$\begin{array}{l}\text { 15. Quantity of inputs applied for the crop plots (quantity/season } \\
\text { for annuals and quantity/year for perennials) for all main and } \\
\text { inter-crops in last } 12 \text { months* }\end{array}$} \\
\hline a. $\quad$ Seeds/Seedlings ('000 Rp spent by household) & & & & & & & & & & \\
\hline b. Manures ('000 Rp spent by household) & & & & & & & & & & \\
\hline c. $\quad$ Chemical fertilizers ('000 Rp spent by household) & & & & & & & & & & \\
\hline d. $\quad$ Pesticides ('000 Rp spent by household) & & & & & & & & & & \\
\hline e. $\quad$ Herbicides ('000 Rp spent by household) & & & & & & & & & & \\
\hline $\begin{array}{l}\text { f. Hired male and female labour on daily basis ('000 Rp spent } \\
\text { by household) }\end{array}$ & & & & & & & & & & \\
\hline g. Hired animal/machine labour ('000 Rp. spent by household) & & & & & & & & & & \\
\hline
\end{tabular}

* Remember that we are not asking for the total cost of inputs/labour used for the crop, but the actual amount spent by the household for the crop. In case of sharecropping, these two values may differ. 


\section{Plantation crops: Plot endowment and production relations (Only OIL PALM / RUBBER)}

5.1. General plot information [A plot is defined as a piece of land under one crop, which is not spatially segmented and where the managerial practices are common and palms/trees are of approximately same age. Complete one column before going to the next.]. How many plots do you own?......

\begin{tabular}{|c|c|c|c|c|}
\hline & Research plot 1 & Plot $2(C 01 / B 09)$ & Plot 3 & Plot 4 \\
\hline 1. Area of plot (ha) & & & & \\
\hline 2. Number of palms/trees in the plot & & & & \\
\hline 3. Number of productive palms/trees in the plot & & & & \\
\hline 4. Do you intercrop the plot? & Yes/No & Yes/No & Yes/No & Yes/No \\
\hline 5. Ownership of land: Owned/Leased-in & Own/Lsd-in & Own/Lsd-in & Own/Lsd-in & Own/Lsd-in \\
\hline 6. Are you employing sharecropping tenants in this plot? (If no, go to question 7) & Yes/No & Yes/No & Yes/No & Yes/No \\
\hline a. If yes, how many of farm households are involved? (number) & & & & \\
\hline b. When did this sharecropping arrangement start for this plot? (year) & & & & \\
\hline c. Does the sharecropping tenant belong to your ethnic group? & $\mathrm{Yes} / \mathrm{No}$ & $\mathrm{Yes} / \mathrm{No}$ & $\mathrm{Yes} / \mathrm{No}$ & Yes/No \\
\hline d. Is the sharecropping tenant your close relative? & $\mathrm{Yes} / \mathrm{No}$ & $\mathrm{Yes} / \mathrm{No}$ & $\mathrm{Yes} / \mathrm{No}$ & Yes/No \\
\hline e. Did you sign a written agreement before starting the sharecropping? & Yes/No & Yes/No & Yes/No & Yes/No \\
\hline f. Input cost $(\%)$ provided by your household & & & & \\
\hline g. Share of output $(\%)$ provided as wage & & & & \\
\hline h. Did the share of output provided as wage increase over last 3 years? & $\mathrm{Yes} / \mathrm{No}$ & Yes/No & Yes/No & Yes/No \\
\hline i. If applicable, was the drop in rubber price the main reason for the increase? & $\mathrm{Yes} / \mathrm{No}$ & $\mathrm{Yes} / \mathrm{No}$ & $\mathrm{Yes} / \mathrm{No}$ & Yes/No \\
\hline
\end{tabular}




\begin{tabular}{|c|c|c|c|c|c|}
\hline & Research plot 1 & Plot $2(C 01 / B 09)$ & Plot 3 & Plot 4 \\
\hline \multicolumn{2}{|c|}{$\begin{array}{l}\text { 11. Was this plot self-established (that is, the household did not obtain an estate } \\
\text { established by someone else)? (If no go to question 11) }\end{array}$} & Yes/No & Yes/No & Yes/No & Yes/No \\
\hline \multicolumn{6}{|c|}{ 12. In case of self-establishment; year of establishment? } \\
\hline \multicolumn{6}{|c|}{$\begin{array}{l}\text { 13. In case plot was not established by the household, how did you acquire the plot? } \\
(\text { Code E) })^{40}\end{array}$} \\
\hline \multicolumn{6}{|c|}{ 14. If at least part of the estate was not established by the household } \\
\hline \multicolumn{6}{|c|}{ a. Year of procurement/purchase } \\
\hline \multicolumn{6}{|c|}{ b. Number of palms/trees already existing in the plot } \\
\hline \multicolumn{6}{|c|}{ c. Age of palms/trees at the time of procurement } \\
\hline \multicolumn{6}{|l|}{ 15. Year of first harvest ever } \\
\hline \multicolumn{6}{|c|}{ 16. Year of last replanting in the plot (put $N A$ if never replanted). } \\
\hline \multicolumn{6}{|c|}{$\begin{array}{l}\text { 17. If replanted, year of first harvest after replanting (if harvesting is not started, } \\
\text { indicate expected year of first harvest) }\end{array}$} \\
\hline \multicolumn{6}{|c|}{ 18. Varieties grown $(1=$ Improved; $0=$ Local $)$} \\
\hline \multicolumn{6}{|l|}{ 19. Distance from the plot to: } \\
\hline \multirow[t]{2}{*}{ a. Home (meters) } & Now & & & & \\
\hline & At the time of establishment of plot & & & & \\
\hline \multirow[t]{2}{*}{ b. Nearest road (meters) } & Now & & & & \\
\hline & At the time of establishment of plot & & & & \\
\hline \multirow[t]{2}{*}{ c. Nearest village center (meters) } & Now & & & & \\
\hline & At the time of establishment of plot & & & & \\
\hline \multicolumn{2}{|c|}{$\begin{array}{l}\text { 20. Have you noticed any land grabbing or land expropriation from any farmers } \\
\text { by government, other farmers, plantation company near this plot? }\end{array}$} & Yes/No & Yes/No & Yes/No & Yes/No \\
\hline $\begin{array}{l}\text { If yes, the year of occurrence o } \\
\text { (e.g., 1998-2001) if the event is }\end{array}$ & $\begin{array}{l}\text { his event }(\mathrm{s}) \text {. A time period may be given } \\
\text { curring over time }\end{array}$ & & & & \\
\hline
\end{tabular}

Code E: transmigrant program $=1$; other government program $=2$; purchased from other farmers $=3$; inherited $=4$; obtained from company $=5$; others (specify) $=6$. 


\subsection{Product marketing: All productive plots of the crop}

a. Number of times output was sold during the last 12 months:......................... (number)

b. Through how many outlets the output was sold during last 12 months:............. (number)

c. During the last 12 months, from how many traders can you choose one for selling output: .................. (maximum number)

\begin{tabular}{|c|c|c|c|c|c|c|}
\hline \multirow{2}{*}{$\begin{array}{l}\text { Outlets where the } \\
\text { output was sold in } \\
\text { last } 12 \text { months } \\
\text { (name) }\end{array}$} & \multirow{2}{*}{$\begin{array}{c}\text { Type of } \\
\text { outlet (Code } \\
\text { A) }\end{array}$} & \multirow{2}{*}{$\begin{array}{l}\text { Do you have } \\
\text { a contract } \\
\text { relation with } \\
\text { outlet? }\end{array}$} & \multirow{2}{*}{$\begin{array}{l}\% \text { of output sold through } \\
\text { this outlet during the last } \\
12 \text { months }\end{array}$} & \multicolumn{3}{|c|}{ Product transport to the outlet point } \\
\hline & & & & $\begin{array}{c}\text { in } \mathrm{km} \\
\text { (0 if purchased at farm- } \\
\text { gate) }\end{array}$ & $\begin{array}{l}\text { mode of } \\
\text { transportation } \\
(\text { Code } \mathrm{B})\end{array}$ & $\begin{array}{c}\text { time taken for } \\
\text { transportation (hours) }\end{array}$ \\
\hline 1. & & Yes/ No & & & & \\
\hline 2. & & Yes/No & & & & \\
\hline 3. & & Yes/No & & & & \\
\hline 4. & & Yes/No & & & & \\
\hline 5. & & Yes/No & & & & \\
\hline 6. & & Yes/No & & & & \\
\hline
\end{tabular}

Code A: private plantation $=1$; government plantation $=2$; private trader in village $=3$; private trader outside village $=4$; farmer group or cooperative $=5$; others (specify) $=6$

Code B: farm-gate selling $=0$; walking $=1 ;$ cycle $=2$; ojek $=3$; angkot $=4$; bus $=5$; truck $=6$; tractor $=7$; others $($ specify $)=8$ 
6. Plot identification

a. Did the farmer start to cultivate the crop after 2012 or is newly added?. (Yes/No) [If farmer started to cultivate the crop after 2012 or the farmer is newly added use additional pages to report information on all new plots in chapter 5.2; 5.3 and 5.4 and proceed to section 6.1]

b. Please let the farmer identify the plots with the data provided from all his plots.

Research Plot: Size:........ ha; Age of plantation:....... years; Distance from home:...........m

Additional C01/B09/Research Plot: Size:........ ha; Age of plantation:....... years; Distance from home:...........m

Additional C01/B09/Research Plot: Size:........ ha; Age of plantation:....... years; Distance from home:...........m

Other Plot: Size:....... ha; Age of plantation:....... years; Distance from home:...........m

Other Plot: Size:....... ha; Age of plantation:....... years; Distance from home:..........m

Other Plot:: Size:....... ha; Age of plantation:...... years; Distance from home:..........m

Other Plot:: Size:....... ha; Age of plantation:....... years; Distance from home:..........m

Other Plot:: Size:....... ha; Age of plantation:....... years; Distance from home:..........m

a. Did the farmer clearly identify the research plot? ..........(Yes/No)

b. If applicable, did the farmer clearly identify the additional C01/B09/Research plot? ..........(Yes/No)

c. If applicable, did the farmer clearly identify the additional C01/B09/Research plot? ...........(Yes/No) 
6.1. Input use during last 12 months: Research plot (Report only data from the identified research plot)

\begin{tabular}{|c|c|c|c|c|c|}
\hline Inputs in research plot & $\begin{array}{l}\text { 1. Name of } \\
\text { input }\end{array}$ & $\begin{array}{l}\text { 2. Number } \\
\text { of times } \\
\text { used }\end{array}$ & $\begin{array}{l}\text { 3. Unit of } \\
\text { measurement }\end{array}$ & $\begin{array}{l}\text { 5. Quantity used } \\
\text { (QU/plot/year) (report in } \\
\text { total and not per times) }\end{array}$ & $\begin{array}{l}\text { 6. Average price of input as used } \\
\text { during last } 12 \text { months ('000 } \\
\mathrm{Rp} / \text { Unit) }\end{array}$ \\
\hline 1. Seedlings (I planting)* & & & Number & & \\
\hline 2. Seedlings (replanting)* & & & Number & & \\
\hline 3. Manure: Plant waste & & & $\mathrm{kg}$ & & \\
\hline 4. Manure: Animal waste & & & $\mathrm{kg}$ & & \\
\hline 5. Soil amendments & Lime / Gypsum & & $\mathrm{kg}$ & & \\
\hline \multirow[t]{5}{*}{ 6. Chemical fertilizers } & & & $\mathrm{kg}$ & & \\
\hline & & & $\mathrm{kg}$ & & \\
\hline & & & $\mathrm{kg}$ & & \\
\hline & & & $\mathrm{kg}$ & & \\
\hline & & & $\mathrm{kg}$ & & \\
\hline \multirow[t]{3}{*}{ 7. Herbicides } & & & litres & & \\
\hline & & & litres & & \\
\hline & & & litres & & \\
\hline \multirow[t]{3}{*}{ 8. Pesticides } & & & litres & & \\
\hline & & & litres & & \\
\hline & & & litres & & \\
\hline 9. Irrigation (excl. labour cost) & & & '000 Rp & & \\
\hline 10. Machinery & & & '000 Rp / liter & & \\
\hline 11. Input transport & & & ‘000 Rp / liter & & \\
\hline 12. Output transport & & & '000 Rp / liter & & \\
\hline $\begin{array}{l}\text { 13. Materials for output processing } \\
\text { in rubber }\end{array}$ & & & ‘000 Rp & & \\
\hline 14. Others (specify) & & & '000 Rp & & \\
\hline
\end{tabular}

*only if planting or replanting was done during the last 12 months 
6.2. Labor use during last 12 months: Research plot

\begin{tabular}{|c|c|c|c|c|c|c|c|c|c|c|c|c|c|}
\hline \multirow[t]{2}{*}{$\begin{array}{l}\text { Labour use in research } \\
\text { plot }\end{array}$} & \multicolumn{2}{|c|}{ 1.a Rainy season } & \multicolumn{2}{|c|}{ 1.b Dry season } & \multirow[t]{2}{*}{$\begin{array}{c}2 . \\
\text { Average } \\
\text { working } \\
\text { hours } \\
\text { per day }\end{array}$} & \multirow[t]{2}{*}{$\begin{array}{c}3 . \\
\text { Contracted } \\
\text { out the } \\
\text { operation? } \\
\text { Yes }=1 / \\
\text { No }=0)\end{array}$} & \multirow[t]{2}{*}{$\begin{array}{l}\text { 4. If } \\
\text { contracted } \\
\text { out, cost of } \\
\text { operation } \\
\text { ('000 Rp) }\end{array}$} & \multicolumn{2}{|c|}{$\begin{array}{c}\text { 5. If not } \\
\text { contracted out, } \\
\text { hired laborers/ } \\
\text { operation } \\
\text { (number) }\end{array}$} & \multicolumn{2}{|c|}{$\begin{array}{c}\text { 6. Wage rate } \\
\text { ('000 Rp/worker } \\
\text { day) } \\
\text { (put sc if } \\
\text { sharecropping) } \\
\end{array}$} & \multicolumn{2}{|c|}{$\begin{array}{l}\text { 7. Family } \\
\text { members } \\
\text { involved/ } \\
\text { operation } \\
\text { (number) }\end{array}$} \\
\hline & $\begin{array}{l}\text { \# of } \\
\text { opera } \\
\text {-tions }\end{array}$ & $\begin{array}{c}\text { Days } \\
\text { per } \\
\text { operation } \\
\end{array}$ & $\begin{array}{l}\text { \# of } \\
\text { opera } \\
\text {-tions } \\
\end{array}$ & $\begin{array}{c}\text { Days } \\
\text { per } \\
\text { operation }\end{array}$ & & & & Men & Women & Men & Women & Men & Women \\
\hline 1. Land clearing for planting & & & & & & & & & & & & & \\
\hline 2. Other pre-planting activities & & & & & & & & & & & & & \\
\hline 3. Taking pits for planting & & & & & & & & & & & & & \\
\hline 4. Seedling transportation & & & & & & & & & & & & & \\
\hline 5. Planting & & & & & & & & & & & & & \\
\hline 6. Replanting & & & & & & & & & & & & & \\
\hline 7. Manure application & & & & & & & & & & & & & \\
\hline 8. Fertilizer application & & & & & & & & & & & & & \\
\hline $\begin{array}{l}\text { 9. Chemical weeding on the } \\
\text { ground }\end{array}$ & & & & & & & & & & & & & \\
\hline $\begin{array}{l}\text { 10. Manual weeding on the } \\
\text { ground }\end{array}$ & & & & & & & & & & & & & \\
\hline 12. Manual weeding on trees & & & & & & & & & & & & & \\
\hline 13. Chemical weeding on trees & & & & & & & & & & & & & \\
\hline 14. Pesticide application & & & & & & & & & & & & & \\
\hline 16. Irrigation & & & & & & & & & & & & & \\
\hline $\begin{array}{l}\text { 17. Intercultural operations } \\
\text { (esp. for soil improvement) }\end{array}$ & & & & & & & & & & & & & \\
\hline 18. Tapping (only for rubber) & & & & & & & & & & & & & \\
\hline 19. Harvesting & & & & & & & & & & & & & \\
\hline 20. Processing of product & & & & & & & & & & & & & \\
\hline 21. Transportation to market & & & & & & & & & & & & & \\
\hline 22. Marketing & & & & & & & & & & & & & \\
\hline 23. Cutting leaves of oil palm & & & & & & & & & & & & & \\
\hline 24. Others (specify) & & & & & & & & & & & & & \\
\hline
\end{tabular}


6.3. Average harvested quantity in the last 12 months: Research plot

\begin{tabular}{|l|c|c|c|}
\hline Season of year & $\begin{array}{c}\text { 1. Frequency of } \\
\text { harvests (once in } \\
\text { how many days?) }\end{array}$ & $\begin{array}{c}\text { 2. Quantity harvested } \\
\text { per month from this } \\
\text { plot (kg/plot) }\end{array}$ & $\begin{array}{c}\text { 3. Average price obtained } \\
\text { for output in that season } \\
\text { ('000 Rp/kg) }\end{array}$ \\
\hline 1. Dry season (June to November) & & & \\
\hline 2. Rainy season (December to May) & & & \\
\hline
\end{tabular}


HH number:

Section 3

\section{Shock events}

7.1. Shock events during the last 3 years: For all plots and crops and livestock

a. Did you suffer from any shocks (e.g. drought, flood, pest) concerning your agricultural activities during the last three years?.

\begin{tabular}{|c|c|c|c|c|c|}
\hline & $\begin{array}{l}\text { 1. Did the } \\
\text { specific shock } \\
\text { occur in the } \\
\text { last three years? }\end{array}$ & $\begin{array}{l}\text { 2. Year and } \\
\text { month of } \\
\text { the shock } \\
\text { event's start } \\
(M M . Y Y Y Y)\end{array}$ & $\begin{array}{l}\text { 3. Year and } \\
\text { month of the } \\
\text { shock event's } \\
\text { end } \\
(M M . Y Y Y Y)\end{array}$ & $\begin{array}{l}\text { 4. Which } \\
\text { plots were } \\
\text { affected? } \\
\text { (Code A) } \\
\text { (Multiple } \\
\text { answers } \\
\text { possible) }\end{array}$ & $\begin{array}{l}\text { 5. How much } \\
\text { of your total } \\
\text { harvest/ } \\
\text { livestock did } \\
\text { you lose in } \\
\text { total over the } \\
\text { whole shock } \\
\text { period? }(\%)\end{array}$ \\
\hline 1. Drought & Yes/No & & & & \\
\hline 2. Too much rain (Flood) & Yes/No & & & & \\
\hline 3. Late rain & Yes/No & & & & \\
\hline 4. Fire & Yes/No & & & & \\
\hline 5. Theft (Eg: Livestock or crops) & Yes/No & & & & \\
\hline 6. Crop pest/disease & Yes/No & & & & \\
\hline 7. Livestock disease & Yes/No & & & & \\
\hline $\begin{array}{l}\text { 8. Critical illness or demise of } \\
\text { HH members }\end{array}$ & Yes/No & & & & \\
\hline 9. Other................. & Yes/No & & & & \\
\hline
\end{tabular}

Code A: Main plot reported for oil palm = 1; Main plot reported for rubber = 2; Additionally reported core $/ \mathrm{C} 01 / \mathrm{B} 09$ plot $=3$; Other plot $=4$.

\section{2. $\quad$ Rubber price shock}

Does the household cultivate rubber?

Since 2012 the price of rubber decreased drastically, how did your

household react to the decrease in income?

(PLEASE MAKE SURE THAT THESE EVENTS HAPPENED AS A RESPONSE TO THE

REDUCTION IN THE RUBBER PRICE)

1. Did you decrease your household savings due to income loss from rubber? $(0=\mathrm{No} ; 1=\mathrm{Yes})$

2. Did you sell land to get additional money to cover the income loss? $(0=\mathrm{No} ; 1=\mathrm{Yes})$

3. Did you apply for more credit (from bank, family or friends) to cover the income loss from rubber? $(0=\mathrm{No} ; 1=\mathrm{Yes})$

4. Did you change some of your plantation from rubber to oil palm? $(0=\mathrm{No} ; 1=\mathrm{Yes})$

5. Did you open up new plantations to cover the income loss from rubber? ( $0=\mathrm{No} ; 1=\mathrm{Yes})$

a. If yes, did this include deforestation? $(0=\mathrm{No} ; 1=\mathrm{Yes})$

b. If yes, did you purchase the land? $(0=\mathrm{No} ; 1=\mathrm{Yes})$

6. Did you start or increase wage labour to cover the income loss from rubber? ( $0=\mathrm{No} ; 1=\mathrm{Yes})$

a. If yes, did you start working in an oil palm plantation or company? $(0=\mathrm{No} ; 1=\mathrm{Yes})$

7. Did you open an own business to cover the income loss from rubber? $(0=\mathrm{No} ; 1=\mathrm{Yes})$

8. Did you stop tapping the rubber trees? $(0=\mathrm{No} ; 1=\mathrm{Yes})$

a. If stopped tapping, for which period did you stop tapping the rubber trees?

(MM.YY - MM.YY)

b. If stopped tapping, at which price did you stop tapping rubber? ('000 Rp/kg)

c. If stopped tapping, at which price did/would you start again tapping rubber? ('000 Rp/kg)

9. Did you reduce the use of farm inputs? (e.g. fertilizer, herbicides) $(0=$ No; $1=$ Yes)

10. Did you apply any other measures to address income loss from rubber price reduction? Specify

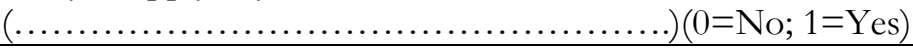


8. Forest dependent activities (Include all the timber and non-timber products your housebold collects or used to collect)

\begin{tabular}{|c|c|c|c|c|c|c|c|c|c|}
\hline \multirow[t]{2}{*}{$\begin{array}{l}\text { 1. Forest product } \\
\text { collected }\end{array}$} & \multirow{2}{*}{$\begin{array}{l}\text { 2. How often } \\
\text { do you collect } \\
\text { it or do it? } \\
\text { (Once in how } \\
\text { many days) }\end{array}$} & \multirow{2}{*}{$\begin{array}{l}\text { 3. How many } \\
\text { members of } \\
\text { your HH are } \\
\text { involved in } \\
\text { collection } \\
\text { (number) }\end{array}$} & \multirow{2}{*}{$\begin{array}{l}\text { 4. How many } \\
\text { other } \\
\text { households are } \\
\text { involved in this } \\
\text { activity? } \\
\text { (number) }\end{array}$} & \multicolumn{2}{|c|}{$\begin{array}{c}\text { 5. Quantity } \\
\text { obtained during } \\
\text { last } 12 \text { months }\end{array}$} & \multicolumn{2}{|c|}{$\begin{array}{l}\text { 6. Quantity sold } \\
\text { during last } 12 \\
\text { months }\end{array}$} & \multirow{2}{*}{$\begin{array}{l}\text { 7. Average } \\
\text { price obtained } \\
\text { (000 Rp/Unit) } \\
\text { during last } 12 \\
\text { months }\end{array}$} & \multirow{2}{*}{$\begin{array}{l}\text { 8. Share of } \\
\text { revenue }(\%) \text { for } \\
\text { your household if } \\
\text { more than } 1 \\
\text { households are } \\
\text { involved }\end{array}$} \\
\hline & & & & $\begin{array}{c}\text { a. } \\
\text { Quantity }\end{array}$ & $\begin{array}{l}\text { b. } \\
\text { Unit }\end{array}$ & $\begin{array}{c}\text { a. } \\
\text { Quantity }\end{array}$ & $\begin{array}{l}\text { b. } \\
\text { Unit }\end{array}$ & & \\
\hline \multicolumn{10}{|l|}{ Timber } \\
\hline \multicolumn{10}{|l|}{ Honey } \\
\hline \multicolumn{10}{|l|}{ Firewood } \\
\hline \multicolumn{10}{|l|}{ Hunting birds in forest } \\
\hline Other hunting & & & & & & & & & \\
\hline Other:............. & & & & & & & & & \\
\hline
\end{tabular}

9. Caged/singing bird questions

- Did you collect any caged (singing) birds in the last 12 months? .......... (Yes/No)

- Is any of your household members breeding singing birds?.................... (Yes/No)

- Are you keeping any caged (singing) birds now in the house? .......... (Yes/No)

- If yes, name of bird type and number.

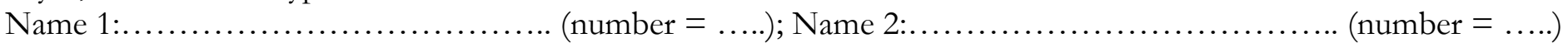
Name $3: \ldots \ldots \ldots \ldots \ldots \ldots \ldots \ldots \ldots \ldots . . \ldots(n u m b e r=\ldots . .$.$) ; Name 4:$

- Were you keeping any other species of caged (singing) birds in the last 3 years?

- If yes, name of bird type..

Name 1:.

; Name 2:.

Name 3: ; Name 4 


\section{Livestock production}

a. Animals possessed and produced by the household during the last 12 months

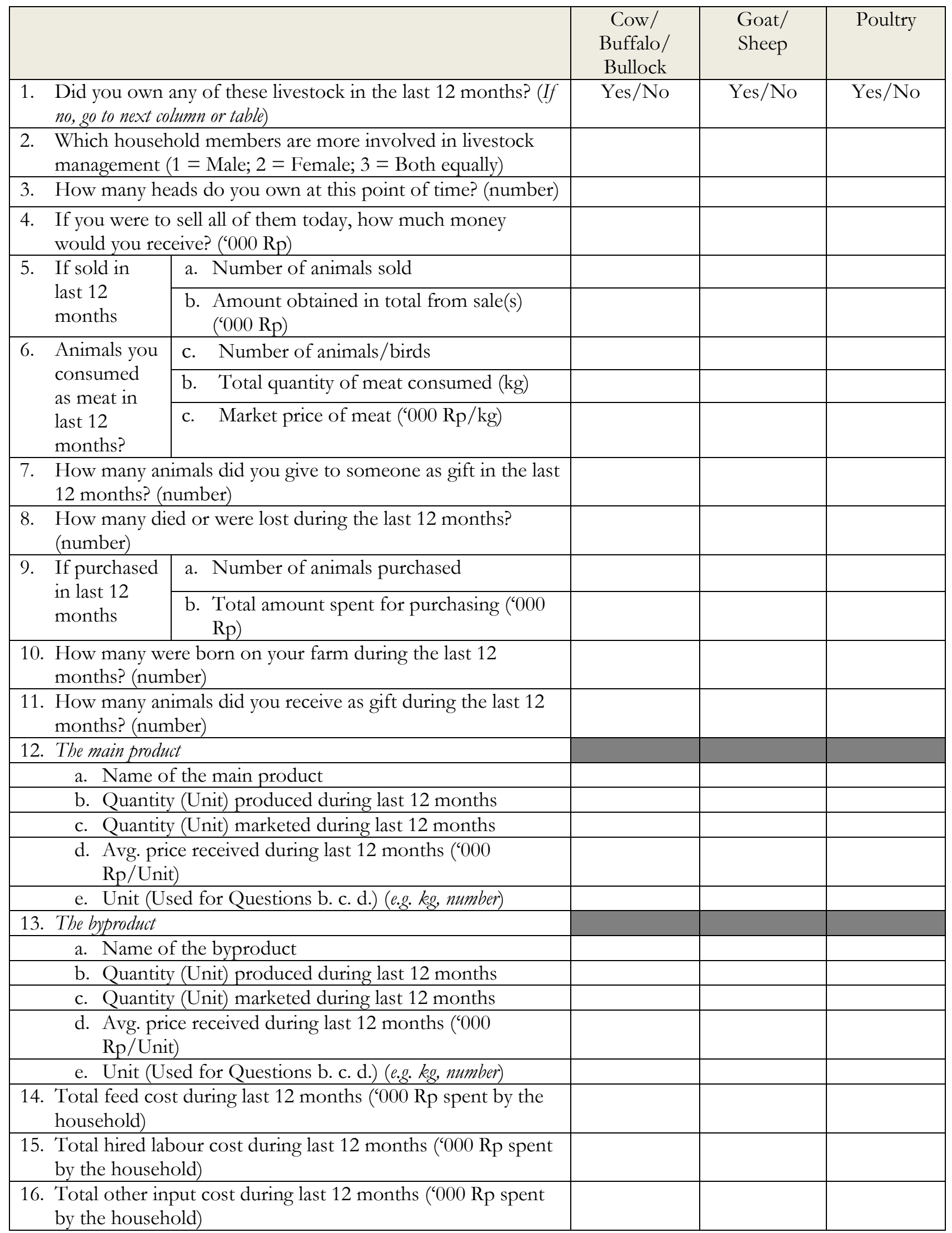




\section{b. Fish culture during the last 12 months}

\begin{tabular}{|c|c|c|c|}
\hline $\begin{array}{l}\text { 1. Have you been involved in fish culture in the last } 12 \text { months? } \\
\text { (If no, go to next table) }\end{array}$ & \multicolumn{3}{|c|}{ Yes/No } \\
\hline \multicolumn{4}{|l|}{$\begin{array}{l}\text { 2. Which household members are more involved in fish culture } \\
(1=\text { Male, } 2=\text { Female; } 3=\text { Both equally })\end{array}$} \\
\hline \multicolumn{4}{|l|}{$\begin{array}{l}\text { 3. Number of households involved in fish cultivation (if done } \\
\text { jointly with others)? }\end{array}$} \\
\hline \multicolumn{4}{|l|}{ 4. Number of ponds under cultivation } \\
\hline \multicolumn{4}{|l|}{ 5. Total size of all fish ponds under cultivation $\left(\mathrm{m}^{2}\right)$} \\
\hline & Fish type 1 & Fish type 2 & Fish type 3 \\
\hline \multicolumn{4}{|l|}{ 6. Name of major fish types being grown } \\
\hline \multicolumn{4}{|l|}{ 7. How many times did you harvest during the last 12 months? } \\
\hline \multicolumn{4}{|l|}{ 8. What is the average quantity of fish obtained per harvest $(\mathrm{kg})$ ? } \\
\hline 9. Did you sell fish? & Yes/No & Yes/No & Yes/No \\
\hline \multicolumn{4}{|l|}{ 10. Amount of fish sold during last 12 months $(\mathrm{kg})$ ? } \\
\hline \multicolumn{4}{|l|}{ 11. If sold, average price obtained ('000 Rp/ kg)? } \\
\hline \multicolumn{4}{|l|}{$\begin{array}{l}\text { 12. How much did you spend on fish feed during the last } 12 \\
\text { months ('000 Rp)? }\end{array}$} \\
\hline \multicolumn{4}{|l|}{$\begin{array}{l}\text { 13. How much did you spend on non-feed materials during the last } \\
12 \text { months (' } 000 \mathrm{Rp} \text { )? }\end{array}$} \\
\hline $\begin{array}{l}\text { 14. How much did you pay for hired labour during last } 12 \text { months } \\
\text { ('000 Rp)? }\end{array}$ & & & \\
\hline
\end{tabular}

\section{c. Fishing during the last 12 months}

\begin{tabular}{|l|c|}
\hline $\begin{array}{l}\text { 1. Apart from fish pond cultivation, do you or any of your HH members go } \\
\text { fishing? }\end{array}$ & $\begin{array}{c}\text { Yes/No } \\
\text { (if no, go to next table) }\end{array}$ \\
\hline 2. How many of your HH members go for fishing? (number) & Yes/No \\
\hline 3. Are female household members involved in fishing? & \\
\hline 4. & How often do you or your HH members go fishing? (once in .....days) \\
\hline 5. How much time do you spend on average when you go fishing (hours/day)? & \\
\hline 6. What is the quantity of fish you obtain in an average month? (kg) & \\
\hline 7. & What is the quantity of fish you sell in an average month? (kg) \\
\hline 8. How much money did you receive from fishing in an average month? ('000 \\
Rp)
\end{tabular}




\section{Credit and Savings}

\subsection{Formal credit institutions}

a. Have you taken or payed back credit during the last 12 months from a bank, farmer group or cooperative?........ (Yes/No)

b. If yes, type of the institute (Code: Bank $=1 ;$ farmer group $=2 ;$ farmer cooperative $=3$, Other $=4$ )

c. If no, what was the main reason for not taking credit?

[Code: Not required or necessary $=1$; Can easily obtain from friends or family $=2$; It is difficult to get $=3$; High interest rate $=4$; No land title to pledge to get credit $=5$; It is morally wrong to take credit $=6$;

Others $=7$ (specify: ..)]

If credit was taken or being paid back in the last 12 months from a bank/farmer group/cooperative/other formal groups:

\begin{tabular}{|c|c|c|c|c|c|c|}
\hline & 1. Bank & $\begin{array}{l}\text { 2.Coopera } \\
\text { tive }\end{array}$ & $\begin{array}{l}\text { 3. Farmer } \\
\text { group }\end{array}$ & 4. Others \\
\hline \multicolumn{7}{|c|}{ 1. Amount taken ('000 Rp) } \\
\hline \multirow{2}{*}{\multicolumn{2}{|c|}{$\begin{array}{l}\text { 2. In which of the } \\
\text { household members' } \\
\text { name the credit was taken }\end{array}$}} & $\begin{array}{l}\text { a. Relationship with } \\
\text { HoH (Code A) }\end{array}$ & & & & \\
\hline & & b. Gender (Code B) & & & & \\
\hline \multicolumn{7}{|c|}{ 3. Date of obtaining credit (DD/MM/YY) } \\
\hline \multicolumn{7}{|c|}{ Interest payment } \\
\hline 4. If interest rate: & \multicolumn{2}{|c|}{ Rate of interest ( $\%$ annual) } & & & & \\
\hline \multirow[t]{2}{*}{ 5. If fixed amount: } & \multicolumn{2}{|c|}{ a. Amount per time ('000 Rp) } & & & & \\
\hline & \multicolumn{2}{|c|}{ b. Number of times per year } & & & & \\
\hline \multicolumn{7}{|c|}{ 6. Repayment period (months) } \\
\hline \multicolumn{7}{|c|}{ 7. $\%$ of credit used for consumption } \\
\hline \multicolumn{7}{|c|}{ 8. $\%$ of credit used for farming } \\
\hline \multicolumn{7}{|c|}{ 9. If used for farming, } \\
\hline \multicolumn{7}{|c|}{ a. $\%$ used for oil palm } \\
\hline \multicolumn{7}{|c|}{ b. $\%$ used for rubber } \\
\hline \multicolumn{3}{|c|}{$\begin{array}{l}\text { 10. Did you have to submit your land } \\
\text { title/certificate to get the credit? }\end{array}$} & Yes / No & Yes / No & Yes / No & Yes / No \\
\hline \multicolumn{3}{|c|}{$\begin{array}{l}\text { 11. Did you have to submit your house } \\
\text { title/certificate to get the credit? }\end{array}$} & Yes / No & Yes / No & Yes / No & Yes / No \\
\hline
\end{tabular}

Code A: household head or wife $=1$; son or daughter $=2$; father or mother $=3$; grandchild $=4$; mother or father in law $=5$; son or daughter in law $=6$; brother $/$ sister $=7$; other relative $=8$; non-relative $=9$.

Code B: male $=1$; female $=2$. 


\subsection{Informal credit sources}

a. Have you taken credit payed back credit during the last 12 months from other households/ trader/ input dealer? ........... (Yes/ No)

b. If yes, type of the institute . (Code: Other household $=1 ;$ Trader $=2$; Input dealer $=3)$

c. If no, what was the main reason for not taking credit?

[Code: Not required or necessary $=1$; Can easily obtain from banks or other formal source $=2$; It is difficult to get $=3$; High interest rate $=4$; No land title to pledge to get credit $=5$; It is morally wrong to take credit $=6$; Others $=7$ (specify: ..)]

\section{If credit was taken or being paid back in the last 12 months from trader/ dealer:}

\begin{tabular}{|c|c|c|c|c|c|}
\hline & \multicolumn{3}{|c|}{ Traders of output } \\
\hline & & & Trader 1 & Trader 2 & Trader 3 \\
\hline \multicolumn{6}{|c|}{ 1. Name of the trader who provides credit } \\
\hline \multirow{2}{*}{\multicolumn{2}{|c|}{$\begin{array}{l}\text { 2. In which household } \\
\text { members' name the credit } \\
\text { was taken }\end{array}$}} & $\begin{array}{l}\text { a. Relationship with } \mathrm{HoH} \\
\text { (Code A) }\end{array}$ & & & \\
\hline & & b. Gender (Code B) & & & \\
\hline \multicolumn{6}{|c|}{ 3. Output handled by the trader (Code C) } \\
\hline \multicolumn{6}{|c|}{ 4. Total credit amount taken in last 12 months ('000 Rp) } \\
\hline \multicolumn{6}{|l|}{ Interest payment } \\
\hline \multicolumn{3}{|l|}{ 5. If interest rate: } & & & \\
\hline \multirow[t]{2}{*}{ 6. If fixed amount: } & a. $\mathrm{An}$ & it per time ('000 Rp) & & & \\
\hline & b. $\mathrm{N}$ & er of times per year & & & \\
\hline \multicolumn{6}{|c|}{ 7. Mutually agreed repayment period (months) } \\
\hline \multicolumn{3}{|c|}{$\begin{array}{l}\text { 8. Does the repayment take place through a reduction in the } \\
\text { product price (against repayment in cash)? }\end{array}$} & Yes / No & Yes / No & Yes / No \\
\hline \multicolumn{3}{|c|}{ 9. $\%$ of credit used for consumption } & & & \\
\hline \multicolumn{3}{|c|}{ 10. $\%$ of credit used for farming } & & & \\
\hline \multicolumn{3}{|c|}{ 11. If used for farming, } & & & \\
\hline \multicolumn{3}{|c|}{ a. $\%$ used for oil palm } & & & \\
\hline \multicolumn{3}{|c|}{ b. $\%$ used for rubber } & & & \\
\hline \multicolumn{3}{|c|}{$\begin{array}{l}\text { 12. Did you have to submit your land title/certificate to } \\
\text { get the credit? }\end{array}$} & Yes / No & Yes / No & Yes / No \\
\hline
\end{tabular}

Code A: household head or wife $=1$; son or daughter $=2$; father or mother $=3$; grandchild $=4$; mother or father in law $=5$; son or daughter in law $=6$; brother $/$ sister $=7$; other relative $=8$; non-relative $=9$.

Code B: male $=1$; female $=2$.

Code C: oil palm $=1$; rubber $=2$; rice $=3$; other $($ specify $)=4$ 
If credit was taken or being paid back in the last 12 months from other households/ other informal sources:

\begin{tabular}{|c|c|c|c|c|c|c|}
\hline & \multicolumn{4}{|c|}{ Other household (major credit sources) } \\
\hline & & & $\mathrm{HH} 1$ & $\mathrm{HH} 2$ & $\mathrm{HH} 3$ & $\mathrm{HH} 4$ \\
\hline \multirow{2}{*}{\multicolumn{2}{|c|}{$\begin{array}{l}\text { 1. Which of the household } \\
\text { member took the initiative to } \\
\text { obtain credit? }\end{array}$}} & $\begin{array}{l}\text { a. Relationship with } \\
\text { HoH (Code A) }\end{array}$ & & & & \\
\hline & & b. Gender (Code B) & & & & \\
\hline \multicolumn{7}{|c|}{ 2. Total amount taken in last 12 months ('000 Rp) } \\
\hline \multicolumn{7}{|l|}{ Interest payment } \\
\hline 3. If interest rate: & Rate o & interest ( $\%$ annual) & & & & \\
\hline \multirow[t]{2}{*}{ 4. If fixed amount: } & a. Amou & t per time ('000 Rp) & & & & \\
\hline & b. Numb & ef of times per year & & & & \\
\hline \multicolumn{7}{|c|}{$\begin{array}{l}\text { 5. Mutually agreed repayment period (months; NA if not } \\
\text { fixed) }\end{array}$} \\
\hline \multicolumn{7}{|c|}{ 6. His/her farm size (ha; 0 if non-farmer) } \\
\hline \multicolumn{7}{|c|}{$\begin{array}{l}\text { 7. Shortest distance between your farm and his/her farm } \\
(\mathrm{km} \text {; NA if not a farmer) }\end{array}$} \\
\hline \multicolumn{3}{|c|}{ 8. Is she/he your relative or friend? } & Yes / No & Yes / No & Yes / No & Yes / No \\
\hline \multicolumn{3}{|c|}{ 9. Does she/he belong to your village? } & Yes / No & Yes / No & Yes / No & Yes / No \\
\hline \multicolumn{3}{|c|}{ 10. Does she/he belong to your dusun? } & Yes / No & Yes / No & Yes / No & Yes / No \\
\hline \multicolumn{7}{|c|}{ 11. What is the distance between your houses? $(\mathrm{km})$} \\
\hline \multicolumn{3}{|c|}{ 12. Do you both belong to same ethnic community? } & Yes/ No & Yes $/ \mathrm{No}$ & Yes / No & Yes / No \\
\hline \multicolumn{3}{|c|}{$\begin{array}{l}\text { 13. Did he/she borrow money from you in past } 12 \\
\text { months? }\end{array}$} & Yes / No & Yes / No & Yes / No & Yes / No \\
\hline \multicolumn{3}{|c|}{ 14. $\%$ of credit used for consumption } & & & & \\
\hline \multicolumn{3}{|c|}{ 15. $\%$ of credit used for farming } & & & & \\
\hline \multicolumn{7}{|l|}{ 16. If used for farming, } \\
\hline \multicolumn{7}{|c|}{ a. $\%$ used for oil palm } \\
\hline \multicolumn{3}{|c|}{ b. $\%$ used for rubber } & & & & \\
\hline \multicolumn{3}{|c|}{$\begin{array}{l}\text { 17. Did you have to submit your land title/certificate } \\
\text { to get the credit? }\end{array}$} & Yes / No & Yes / No & Yes / No & Yes / No \\
\hline
\end{tabular}

Code A: household head or wife $=1$; son or daughter $=2$; father or mother $=3$;

grandchild $=4$; mother or father in law $=5$; son or daughter in law $=6$; brother $/$ sister $=7$; other relative $=8$; non-relative $=9$.

Code $\mathbf{B}$ : male $=1$; female $=2$; joint $=3$.

\subsection{Savings}

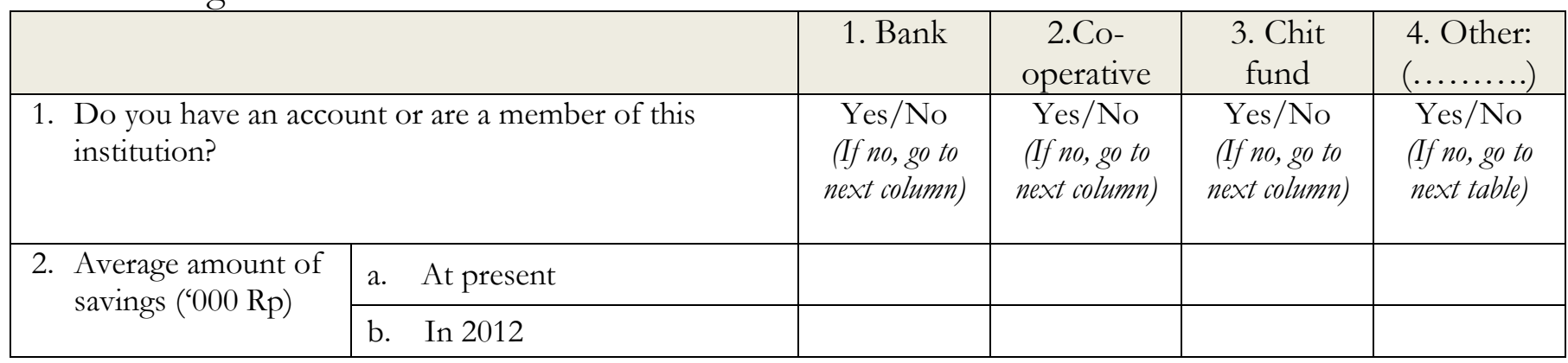




\section{Household characteristics}

\subsection{Asset baseline}

a. When was the household established? .. (year)

\begin{tabular}{|c|c|c|}
\hline & \multicolumn{2}{|c|}{ Number of items owned } \\
\hline & $\begin{array}{l}\text { in } 1990 \text { or time of household establishment (if } \\
\text { established after 1990)? }\end{array}$ & at this point of time \\
\hline 1. Television (colour) & & \\
\hline 2. Satellite Dish & & \\
\hline 3. Motorbike & & \\
\hline 4. $\mathrm{Car}$ & & \\
\hline 5. 4-wheel tractor & & \\
\hline 6. Jeep/Truck & & \\
\hline 7. Fridge & & \\
\hline 8. Air conditioner (AC) & & \\
\hline 9. Washing machine & & \\
\hline
\end{tabular}

\subsection{Asset accumulation}

a. Number of cellphones owned by the household in the present:.........

\begin{tabular}{|c|c|c|c|c|c|c|}
\hline & $\begin{array}{l}\text { Number of items } \\
\text { owned in last } 25 \text { years }\end{array}$ & $\begin{array}{l}\text { Year of } \\
\text { ownership }\end{array}$ & $\begin{array}{l}\text { Purchase } \\
\text { price }\end{array}$ & $\begin{array}{l}\text { Year } \\
\text { sold/lost }\end{array}$ & $\begin{array}{r}\text { If was regis } \\
\text { whose }\end{array}$ & $\begin{array}{l}\text { red, under } \\
\text { ame? }\end{array}$ \\
\hline & or from $\mathrm{HH}$ estabt. & /purchase & ('000 Rp) & $\begin{array}{l}\text { (Put NA if } \\
\text { still owned) }\end{array}$ & $\begin{array}{l}\text { Relationship } \\
\text { with } \mathrm{HoH} \\
(\text { Code A })^{41}\end{array}$ & $\begin{array}{c}\text { Gender } \\
(1=\text { male; } \\
2=\text { female })\end{array}$ \\
\hline 1. Televisi & & & & & & \\
\hline (colour) & & & & & & \\
\hline & & & & & & \\
\hline 2. Satellite & & & & & & \\
\hline & & & & & & \\
\hline & & & & & & \\
\hline & & & & & & \\
\hline 3. Motorbi & & & & & & \\
\hline 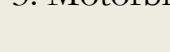 & & & & & & \\
\hline & & & & & & \\
\hline & & & & & & \\
\hline 4. Car & & & & & & \\
\hline & & & & & & \\
\hline & & & & & & \\
\hline 5. 4-wheel & & & & & & \\
\hline tractor & & & & & & \\
\hline & & & & & & \\
\hline
\end{tabular}

Code A: not registered $=0$; household head or wife $=1$; son or daughter $=2$; father or mother $=3$; grandchild $=4$; mother or father in law $=5$; son or daughter in law $=6$; brother $/$ sister $=7$; other relative $=8$; non-relative $=9$. 
Section 3

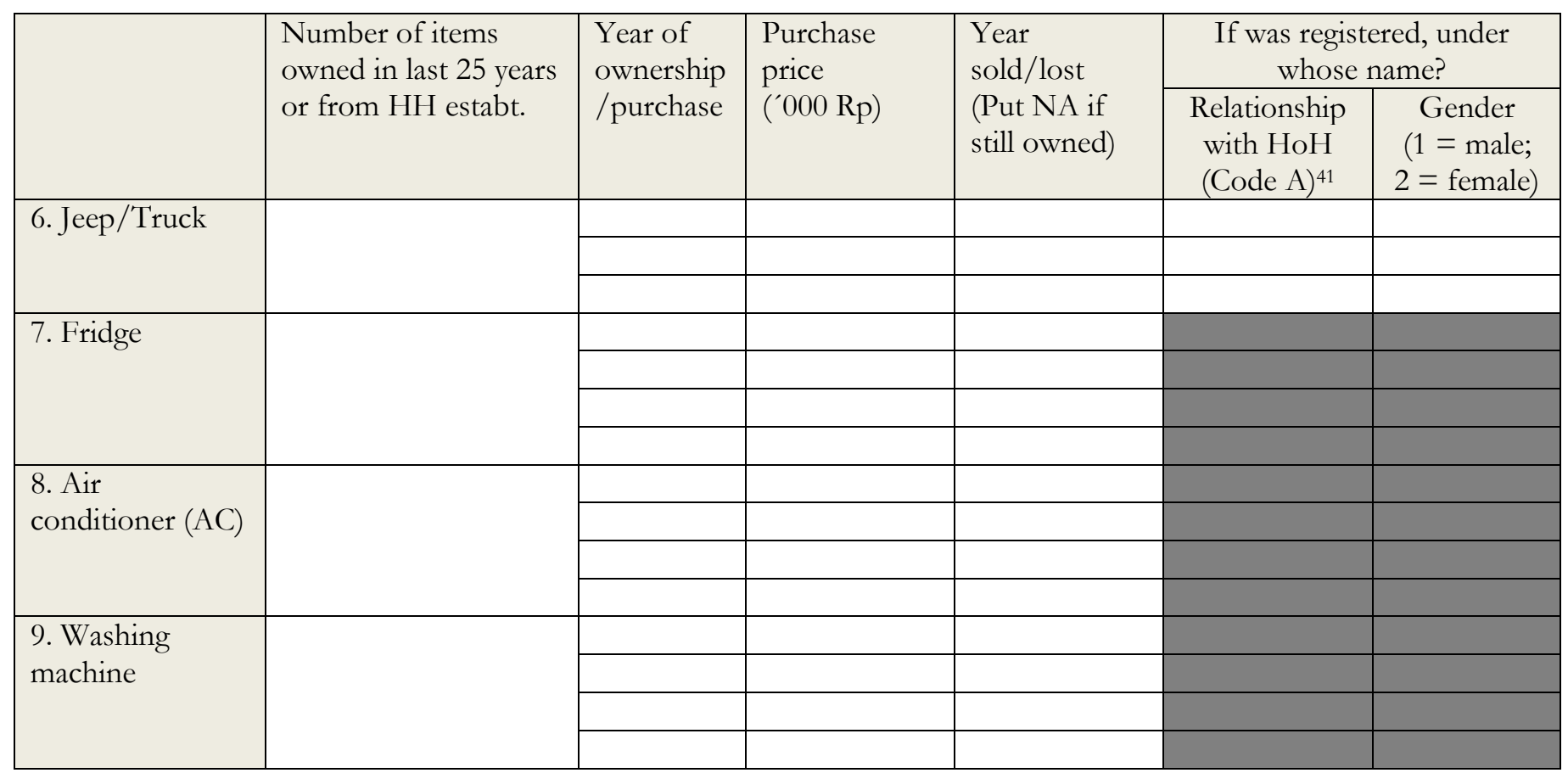

Code A: not registered=0; household head or wife $=1$; son or daughter $=2$; father or mother $=3$; grandchild=4; mother or father in law $=5$; son or daughter in law $=6$; brother $/$ sister $=7$; other relative $=8$; non-relative $=9$. 


\subsection{Housing}

a. Did you build or purchase a house in the last 25 years? (Yes/No)

b. If yes, in which year: .............

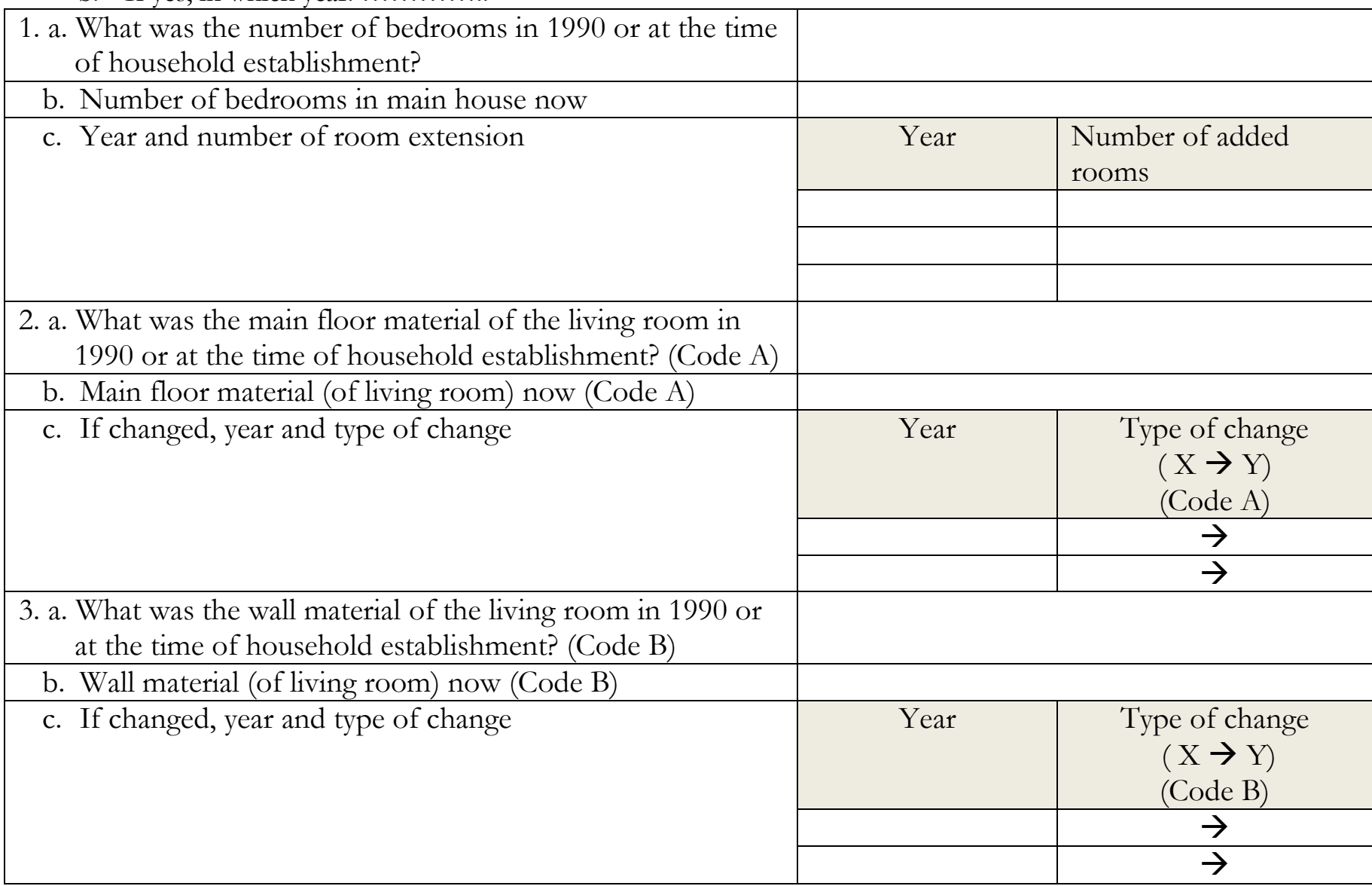

Code A: Tiles $=1$; Cement $=2$; Wood=3; Earth $=4$; Other ( specify) $=5$.

Code B: Un-plastered brick $=1$; Brick covered with cement $=2$; Brick with ceramics $=3$; Low quality wood $=4$, High quality wood (e.g. ornamentation $)=5$; Other (specify $)=6$. 
12.4. Household member details

Details of household members.

a. Total members in the household staying in the house:............... (number) during the last 12 months.

b. Religion of HoH: Muslim/ Christian/ Hindu/ Buddhist/ Others (specify: .......................)

c. Ethnic group (specify):

\begin{tabular}{|c|c|c|c|c|c|c|c|c|}
\hline \multirow{2}{*}{$\begin{array}{l}\text { 1. HH member } \\
\text { (Relationship with } \mathrm{HoH} \text { ) }\end{array}$} & \multirow{2}{*}{$\begin{array}{l}2 . \\
\text { Member } \\
\text { ID }\end{array}$} & \multirow{2}{*}{$\begin{array}{l}\text { 3. Age } \\
\text { (years) }\end{array}$} & \multirow{2}{*}{$\begin{array}{l}\text { 4. Sex } \\
(\mathrm{m} / \mathrm{f})\end{array}$} & \multirow{2}{*}{$\begin{array}{l}\text { 5. Marital status } \\
\text { (married }=1 \text {, } \\
\text { Unmarried }=0 \text { ) }\end{array}$} & \multirow{2}{*}{$\begin{array}{c}\text { 6. Education (number } \\
\text { of years in school and } \\
\text { college) }\end{array}$} & \multirow{2}{*}{$\begin{array}{c}7 . \text { Last } \\
\text { graduation } \\
\text { (Code A) }\end{array}$} & \multicolumn{2}{|c|}{ 8. Main Occupations (Code B) } \\
\hline & & & & & & & a. Primary & b. Secondary \\
\hline Respondent & 1 & & & & & & & \\
\hline \multirow[t]{14}{*}{ Head of household* } & 2 & & & & & & & \\
\hline & 3 & & & & & & & \\
\hline & 4 & & & & & & & \\
\hline & 5 & & & & & & & \\
\hline & 6 & & & & & & & \\
\hline & 7 & & & & & & & \\
\hline & 8 & & & & & & & \\
\hline & 9 & & & & & & & \\
\hline & 10 & & & & & & & \\
\hline & 11 & & & & & & & \\
\hline & 12 & & & & & & & \\
\hline & 13 & & & & & & & \\
\hline & 14 & & & & & & & \\
\hline & 15 & & & & & & & \\
\hline
\end{tabular}

* Do not fill this column if respondent is head of the household. Use more rows if household size is more than 15.

Code A: never attended=1; attended but not completed=2; completed SD (primary)=3; completed SMP $($ Middle $)=4$; completed SMA $($ High School $)=5$; D3 or S1 (Associates Degree or University level first stage) $=6$; student at present $=7$; other (specify) $=8$.

Code B: own-agriculture=1; wage or contract labour=2; own-business activities $=3$; still attending school=4; household activities $=5$; other $($ specify $)=6$. 
b. Residency status of household members (Only ask if household is newly added to sample or household head got married after 2012)

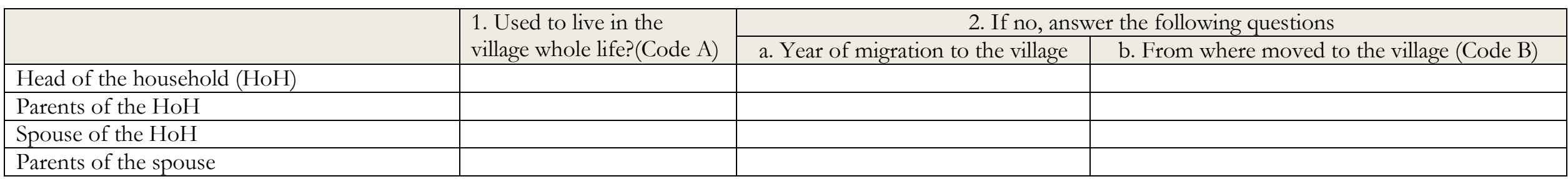

Code A: no $=0$; yes $=1$; never lived in the village $=2$.

Code B: outside village in Jambi = 1; outside Jambi, but in Sumatra = 2; outside Sumatra, but in Indonesia $=3$; outside Indonesia $=4$.

\section{Non-own agriculture household income sources}

\subsection{Wage and contract labour}

a. Have any of your household members worked as a tenant in a sharecropping arrangement in the last 12 months?

If yes: (i). Does the sharecropping landlord belong to your ethnic group? ............. (Yes/No)

(ii). Is the sharecropping landlord your close relative? ............... (Yes/No).

(iii). Did you sign a written agreement before starting the sharecropping? .............. (Yes/No).

(iv). How much does your household (tenant) spend on inputs per month (e.g. fertilizer) for the sharecropped plots? ......('000 Rp)

b. Have any of your household members worked as daily laborer (daily / weekly / monthly payment of money), as contracted for work (fixed payment for specific jobs) or sharecropping tenants during the last 12 months? .............. (Yes/No). (If no, please go to the next table)

\begin{tabular}{|c|c|c|c|c|c|c|c|}
\hline $\begin{array}{l}\text { 1. Member ID (from } \\
\text { Table 12.4a) }\end{array}$ & $\begin{array}{l}\text { 2. Type of } \\
\text { activity } \\
\text { (Code A) }\end{array}$ & $\begin{array}{l}\text { 3. Type of } \\
\text { payment } \\
\text { (Code B) }\end{array}$ & $\begin{array}{l}\text { 4. Seasonal } \\
(=0) \text { or } \\
\text { permanent } \\
(=1) ?\end{array}$ & $\begin{array}{l}\text { 5. If permanent, } \\
\text { wage rate ('000 } \\
\text { Rp/month)* }\end{array}$ & \multicolumn{3}{|c|}{ 6. If seasonal } \\
\hline & & & & & & & \\
\hline & & & & & & & \\
\hline
\end{tabular}

Code A: work in agriculture $=1$; work in forestry $=2$; work in manufacturing $=3$; work in services $=4$; government employee $=5$; other $($ specify $)=6$

Code B: per hour wage $=1$; daily wage $=2$; weekly wage $=3$; monthly wage $=4$; contract (fixed arrangement) $=6$; other arrangement $($ specify $)=7$; share cropping arrangement as tenant $=8$.

* Please estimate the monthly income (deducting the input costs) of household members working as sharecropping tenants 


\subsection{Own business activities}

- Did any of your household members gain any income from any type of own-business activities during the last 12 months?............. (Yes/No) (If no, please go to the next table)

\begin{tabular}{|c|c|c|c|c|c|c|c|}
\hline $\begin{array}{l}\text { 1. Type of } \\
\text { enterprise } \\
\text { or business } \\
\text { (Code A) }\end{array}$ & $\begin{array}{l}\text { 2. Shortly } \\
\text { describe the type } \\
\text { of business in } \\
\text { words }\end{array}$ & $\begin{array}{l}\text { 3. When } \\
\text { started? } \\
\text { (year) }\end{array}$ & $\begin{array}{l}\text { 4. No. of } \\
\text { family } \\
\text { members } \\
\text { engaged }\end{array}$ & $\begin{array}{l}\text { 5. Household member } \\
\text { who is mainly responsible } \\
\text { for the business (ID from } \\
12.4 \mathrm{a} \text { ) }\end{array}$ & $\begin{array}{l}\text { 6. Total hours a } \\
\text { member worked } \\
\text { in business on } \\
\text { average per } \\
\text { month? }\end{array}$ & $\begin{array}{l}\text { 7. Total net amount } \\
\text { (costs subtracted) } \\
\text { earned from business } \\
\text { per month on } \\
\text { average? ('000 Rp) }\end{array}$ & $\begin{array}{l}\text { 8. Number of } \\
\text { months the } \\
\text { business was } \\
\text { running during last } \\
12 \text { months? }\end{array}$ \\
\hline & & & & & & & \\
\hline & & & & & & & \\
\hline & & & & & & & \\
\hline
\end{tabular}

Code A: shop=1; trading=2; restaurant (food)=3; hotel (stay)=4; chauffeur/driver=5; carpenter=6; construction worker=7; other $($ specify $)=8$.

\subsection{Public and private transfers}

- Have any of your household members benefited from some kind of public/NGO transfer program (given money in daily/weekly/monthly basis) during the last 12 months?

\begin{tabular}{|c|c|c|c|c|}
\hline $\begin{array}{c}\text { 1.Member IDs } \\
\text { (from 12.4a) }\end{array}$ & $\begin{array}{c}\text { 2. Type of program } \\
\text { (Code A) }\end{array}$ & $\begin{array}{c}\text { 3. Who is providing the } \\
\text { program? (Code B) }\end{array}$ & $\begin{array}{c}\text { 4. What kind of benefits do you } \\
\text { receive? (Code C) }\end{array}$ & $\begin{array}{c}5 . \text { Estimated amount received } \\
\text { during last } 12 \text { months (‘000 Rp.) }\end{array}$ \\
\hline & & & & \\
\hline & & & & \\
\hline
\end{tabular}

Code A: pensions=1; education subsidies=2; health care benefits=3; poverty reduction program =4; others (specify) $=5$.

Code B: local government $=1$; federal government $=2 ; \mathrm{NGO}=3$; other $($ specify $)=4$.

Code C: cash=1; clothes $=2$; food $=3$; agricultural inputs $=4$; others (specify) $=5$. 


\subsection{Private transfers and remittances}

- Did your household sent any money to anybody (e.g. a family member, not included in 12.4a) staying outside the household during the last 12 months? .......... (Yes/No).

- Did anybody (e.g. a family member, not included in 12.4a) staying outside the household sent money to your household during the last 12 months? ......... (Yes/No). (If no to both questions, go to next table.)

\begin{tabular}{|c|c|c|c|c|c|c|c|c|c|}
\hline & 1. If $\mathrm{mor}$ & is sent outside & & & If money is & ceived from outsid & & 3. Regio & where \\
\hline $\begin{array}{l}\text { a. Receiver's } \\
\text { relation with your } \\
\text { household head }\end{array}$ & $\begin{array}{c}\text { b. } \\
\text { Receiver's } \\
\text { Gender }\end{array}$ & $\begin{array}{l}\text { c. Estimated amount } \\
\text { sent during last } 12 \\
\text { months ('000 Rp.) }\end{array}$ & $\begin{array}{l}\text { d. Main } \\
\text { reasons for } \\
\text { remittance }\end{array}$ & $\begin{array}{l}\text { a. Sender's } \\
\text { relation with } \\
\text { your household }\end{array}$ & $\begin{array}{c}\text { b. } \\
\text { Sender's } \\
\text { Gender }\end{array}$ & $\begin{array}{c}\text { c. Estimated } \\
\text { amount received } \\
\text { during last } 12\end{array}$ & $\begin{array}{l}\text { d. Main } \\
\text { reasons for } \\
\text { remittance }\end{array}$ & $\begin{array}{l}\text { the ser } \\
\text { receive } \\
(\mathrm{Coc}\end{array}$ & $\begin{array}{l}\text { der or } \\
\text { resides } \\
\text { e D) }\end{array}$ \\
\hline & & & & & & & & Receiver & Sender \\
\hline & & & & & & & & & \\
\hline & & & & & & & & & \\
\hline & & & & & & & & & \\
\hline & & & & & & & & & \\
\hline
\end{tabular}

Code A: Son/daughter $=1$; father or mother $=2$; grandchild $=3$; mother or father in law $=4$; son or daughter in law $=5$; other relative $=6$; nonrelative $=7$.

Code B: Male $=1 ;$ Female $=2$.

Code C: emergency spending $=1$; financing education $=2$; supporting livelihood $=3$, other (specify) $=4$.

Code D: outside village in Jambi = 1; outside Jambi, but in Sumatra = 2; outside Sumatra, but in Indonesia $=3$; Outside Indonesia $=4$; same village $=5$.

14. Membership in the village-level organizations in last 12 months

\begin{tabular}{|l|l|l|l|l|l|}
\hline $\begin{array}{l}\text { 1. Household member ID } \\
\text { (see Table 12.4a) }\end{array}$ & $\begin{array}{l}\text { 2. Name of } \\
\text { organization }\end{array}$ & $\begin{array}{l}\text { 3. Position in } \\
\text { organization (other than } \\
\text { being member) }\end{array}$ & $\begin{array}{l}\text { 4. How many } \\
\text { people in the } \\
\text { village participate? }\end{array}$ & $\begin{array}{l}\text { 5. How often do you } \\
\text { meet? (Code A) }\end{array}$ & $\begin{array}{l}\text { 6. Describe functions of the } \\
\text { organisation (Code B) } \\
\text { (Multiple answers allowed) }\end{array}$ \\
\hline & & & & & \\
\hline & & & & & \\
\hline
\end{tabular}

Code A: Each year=1; each half year=2; each quarter=3, each month=4; each week $=5$; no meetings $=6$; other $($ specify $)=7$.

Code B: Religious meetings $=1$; to save jointly $=2$; share experience $=3$; collective purchases of inputs $=4$; collective sales of farm outputs $=5$; plan village related events $=6$; give out credit $=7$; other (specify) $=8$. 
15. History of off-farm income generation activities by the household members (male and female separately)

\begin{tabular}{|c|c|c|c|c|c|c|c|c|}
\hline \multirow[t]{3}{*}{ Year } & \multicolumn{8}{|c|}{ Number of household members involved } \\
\hline & \multicolumn{2}{|c|}{ 1. Working as daily wage labourer } & \multicolumn{2}{|c|}{ 2. Working as permanent labourer } & \multicolumn{2}{|c|}{$\begin{array}{l}\text { 3. Working as labourer in a share } \\
\text { cropping arrangement }\end{array}$} & \multicolumn{2}{|c|}{ 4. Own business activities } \\
\hline & Male & Female & Male & Female & Male & Female & Male & Female \\
\hline 1990 & & & & & & & & \\
\hline 1991 & & & & & & & & \\
\hline 1992 & & & & & & & & \\
\hline 1993 & & & & & & & & \\
\hline 1994 & & & & & & & & \\
\hline 1995 & & & & & & & & \\
\hline 1996 & & & & & & & & \\
\hline 1997 & & & & & & & & \\
\hline 1998 & & & & & & & & \\
\hline 1999 & & & & & & & & \\
\hline 2000 & & & & & & & & \\
\hline 2001 & & & & & & & & \\
\hline 2002 & & & & & & & & \\
\hline 2003 & & & & & & & & \\
\hline 2004 & & & & & & & & \\
\hline 2005 & & & & & & & & \\
\hline 2006 & & & & & & & & \\
\hline 2007 & & & & & & & & \\
\hline 2008 & & & & & & & & \\
\hline 2009 & & & & & & & & \\
\hline 2010 & & & & & & & & \\
\hline 2011 & & & & & & & & \\
\hline 2012 & & & & & & & & \\
\hline 2013 & & & & & & & & \\
\hline 2014 & & & & & & & & \\
\hline 2015 & & & & & & & & \\
\hline
\end{tabular}




\section{Perceptions of land titles and certification programs}

\subsection{Land titles}

a. In the last 15 years, how many conflicts/litigation happened in this village between farmers over land ownership? ...... number

b. Are there any incidents in the village over the last 15 years that the land was taken from a farmer by government agencies or plantation/mining companies using force? . (yes $=1 ;$ no $=0 ;$ NI $=$ No idea $)$

Details on land title documents:

\begin{tabular}{|c|c|c|c|}
\hline & No title & With sporadic & With systematic \\
\hline $\begin{array}{l}\text { 1. What is the maximum amount of credit obtainable from a bank, having one hectare } \\
\text { land with the specific title? (' } 000) \mathrm{Rp}\end{array}$ & & & \\
\hline 2. Price of one hectare of land without plantation but with road access ('000) Rp & & & \\
\hline
\end{tabular}

\subsection{Certification schemes}

\begin{tabular}{|c|c|c|}
\hline & $\begin{array}{c}\text { ISPO } \\
\text { Indonesian Sustainable Palm Oil } \\
\text { Certification }\end{array}$ & $\begin{array}{c}\text { RSPO } \\
\text { Roundtable on Sustainable } \\
\text { Palm Oil Certification }\end{array}$ \\
\hline $\begin{array}{l}\text { 1. Are you familiar with the name of the certification schemes? } \\
(1=\text { yes; } 0=\text { no) (If no, go to next column } / \text { section) }\end{array}$ & & \\
\hline 2. How have you heard about the schemes? (Code A) & & \\
\hline $\begin{array}{l}\text { 3. Are you currently involved in any of these certification schemes? } \\
(1=\text { yes; } 0=\text { no) (If no, go to next column/ section) }\end{array}$ & & \\
\hline 4. If yes, since which year are you involved? & & \\
\hline 5. If yes, what was the main reason for your involvement? (Code B) & & \\
\hline
\end{tabular}

Code A: Other farmers = 1; Processing companies = 2; Government agencies = 3; Non-Governmental organizations = 4; Mass media = 5; Others $($ specify $)=6$.

Code B: Requirement by processing company $=1$; legal requirement $=2$; expecting higher prices $=3$; expecting higher output $=4$; got external support for

implementing scheme $=5$; health benefits $=6$; others (specify) $=7$ 


\section{Decision-making}

Who is primarily responsible for the following consumption expenditure items and tasks?

\begin{tabular}{|c|c|c|c|c|}
\hline \multirow[t]{2}{*}{ Consumption and task items } & \multirow[t]{2}{*}{ Example } & \multicolumn{3}{|c|}{ Persons responsible (Put $\sqrt{ }$ mark or NA if not applicable) } \\
\hline & & Male members & Female members & Both male and female members equally \\
\hline 1. Purchase of food items & Rice, vegetables, meat etc. & & & \\
\hline 2. Paying the bills & Telephone, electricity, gas etc. & & & \\
\hline $\begin{array}{l}\text { 3. Selecting clothing and } \\
\text { footwear }\end{array}$ & Cloths, tailoring, footwear etc. & & & \\
\hline $\begin{array}{l}\text { 4. Paying for recreation and } \\
\text { membership }\end{array}$ & Movies etc. & & & \\
\hline $\begin{array}{l}\text { 5. Spending on education of } \\
\text { children (if applicable) }\end{array}$ & School fees, books etc. & & & \\
\hline 6. Travel and transport & Taxi, public bus etc. & & & \\
\hline $\begin{array}{l}\text { 7. Purchase and sale of } \\
\text { durable goods }\end{array}$ & Purchasing television etc. & & & \\
\hline $\begin{array}{l}\text { 8. Purchasing and sale of land } \\
\text { and houses }\end{array}$ & Including involving in sharecropping & & & \\
\hline $\begin{array}{l}\text { 9. Representing the } \\
\text { household in the public }\end{array}$ & $\begin{array}{l}\text { Talking to officials, participating in } \\
\text { the discussions and group meetings etc. }\end{array}$ & & & \\
\hline
\end{tabular}




\section{Household Survey Questionnaire C07}

(Consumption; Round 2, 2015)

1. Household identification

\begin{tabular}{|c|c|c|}
\hline 1. Village (name): & & \\
\hline 2. Dusun (name or number): & & \\
\hline 3. RT (number): & & \\
\hline 4. Household code (given by supervisor): & & \\
\hline 5. Name of respondent: & & \\
\hline 6. Did the respondent change since 2012? & \multirow{2}{*}{\multicolumn{2}{|c|}{ Yes/No }} \\
\hline 7. Why did the respondent change? (Code $\mathrm{A})$ & & \\
\hline 8. Gender of respondent: & \multicolumn{2}{|c|}{ Male / Female } \\
\hline 9. Name of head of household: & & \\
\hline $\begin{array}{l}\text { 10. Respondent's relationship with head of } \\
\text { household (Code B): }\end{array}$ & & \\
\hline $\begin{array}{l}\text { 11. Number of persons regularly consuming } \\
\text { food from your house in last } 7 \text { days: }\end{array}$ & & \\
\hline 12. Interviewer (name): & & \\
\hline 13. Supervisor (name): & & \\
\hline 14. Date of interview & ..................../2015 & $\begin{array}{l}\text { Enumerator's } \\
\text { signature: }\end{array}$ \\
\hline $\begin{array}{l}\text { 15. Date questionnaire was checked by } \\
\text { supervisor: }\end{array}$ & .........../........../2015 & $\begin{array}{l}\text { Supervisor's } \\
\text { signature: }\end{array}$ \\
\hline
\end{tabular}

Code A: currently out of village $=1$, moved out of the household=2; passed away=3; other (specify)= 4

Code B: Wife $/$ Husband $=1$; Daughter $/$ Son $=2 ;$ Mother $/$ Father $=3$; Sister $/$ Brother $=4$;

Niece $/$ Nephew $=5$; Others (specify) $=6$

2. Household expenditure: In the following questions, we want to ask about all items consumed in your household, regardless of which person consumed it.

2.1. Weekly consumption: Has your household consumed following goods during the past 7 days? Please exclude from your answer any purchases for processing or resale in a household enterprise.

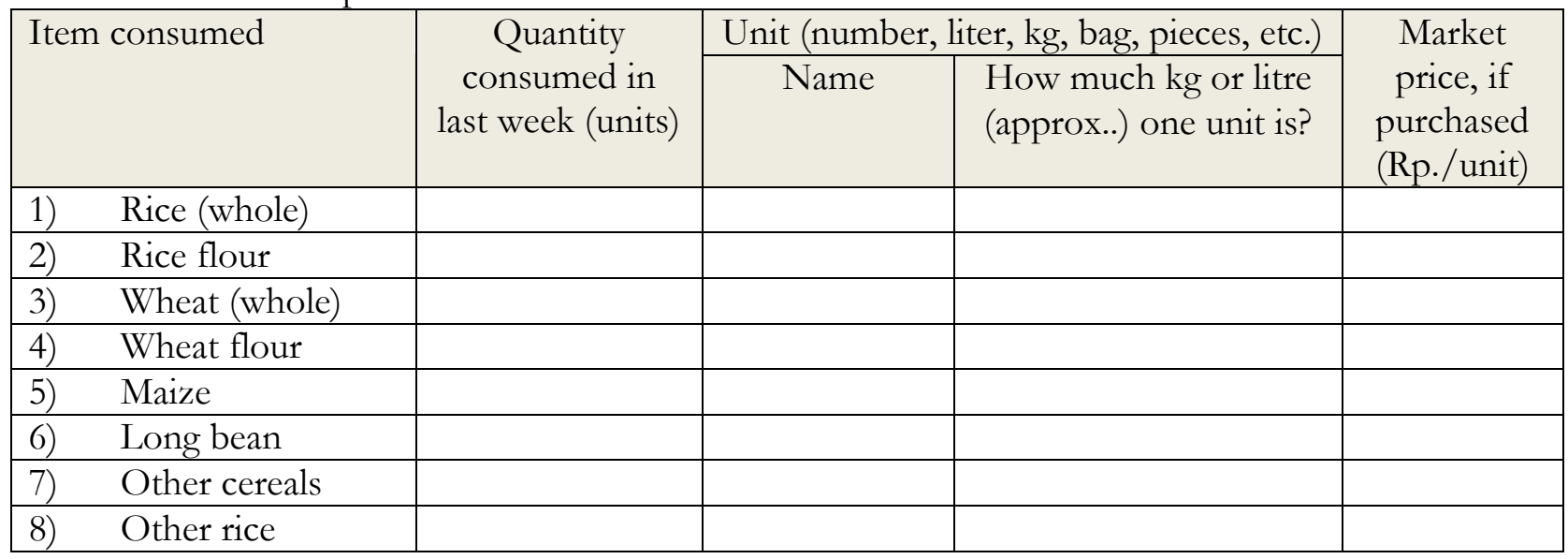




\begin{tabular}{|c|c|c|c|c|c|}
\hline & \multirow{2}{*}{$\begin{array}{c}\text { Quantity } \\
\text { consumed in } \\
\text { last week (units) }\end{array}$} & \multirow{2}{*}{\multicolumn{2}{|c|}{ Unit (number, liter, kg, bag, pieces, etc.) }} & \multirow{2}{*}{$\begin{array}{c}\text { Market } \\
\text { price, if } \\
\text { purchased } \\
\text { (Rp./unit) }\end{array}$} \\
\hline & & & & & \\
\hline \multicolumn{2}{|c|}{ Item consumed } & & & & \\
\hline 10) & Flour of cassava & & & & \\
\hline 11) & Potato & & & & \\
\hline 12) & Sweet potato & & & & \\
\hline 13) & Gaplek & & & & \\
\hline 14) & Taro & & & & \\
\hline 15) & Sago & & & & \\
\hline 16) & Fish (fresh) & & & & \\
\hline 17) & Fish (dry) & & & & \\
\hline 18) & Seafood & & & & \\
\hline 19) & Beef & & & & \\
\hline 20) & Chicken & & & & \\
\hline 21) & Duck & & & & \\
\hline 22) & Mutton & & & & \\
\hline 23) & Buffalo & & & & \\
\hline 24) & Goat & & & & \\
\hline 25) & Lamb & & & & \\
\hline 26) & Sheep & & & & \\
\hline 27) & Entrails & & & & \\
\hline 28) & Liver & & & & \\
\hline 29) & Spleen & & & & \\
\hline 30$)$ & Dried jerky meat & & & & \\
\hline 31) & Eggs of chicken & & & & \\
\hline 32) & Eggs of goose & & & & \\
\hline 33) & Eggs of quail & & & & \\
\hline 34) & Fresh Milk & & & & \\
\hline 35$)$ & Milk powder & & & & \\
\hline 36) & Condensed milk & & & & \\
\hline 38$)$ & Water spinach & & & & \\
\hline 39) & $\begin{array}{l}\text { Land spinach and } \\
\text { Cassava leaves }\end{array}$ & & & & \\
\hline 40) & Cucumber & & & & \\
\hline 41) & Carrots & & & & \\
\hline 42) & Sprout & & & & \\
\hline 43) & String bean & & & & \\
\hline 44) & Garlic & & & & \\
\hline 45) & Chili & & & & \\
\hline 46) & Tomato & & & & \\
\hline 47) & Onion & & & & \\
\hline 48) & Bitter gourd & & & & \\
\hline 49) & Eggplant & & & & \\
\hline 50$)$ & Cabbage & & & & \\
\hline 51) & Beans & & & & \\
\hline 52) & Peanut & & & & \\
\hline
\end{tabular}




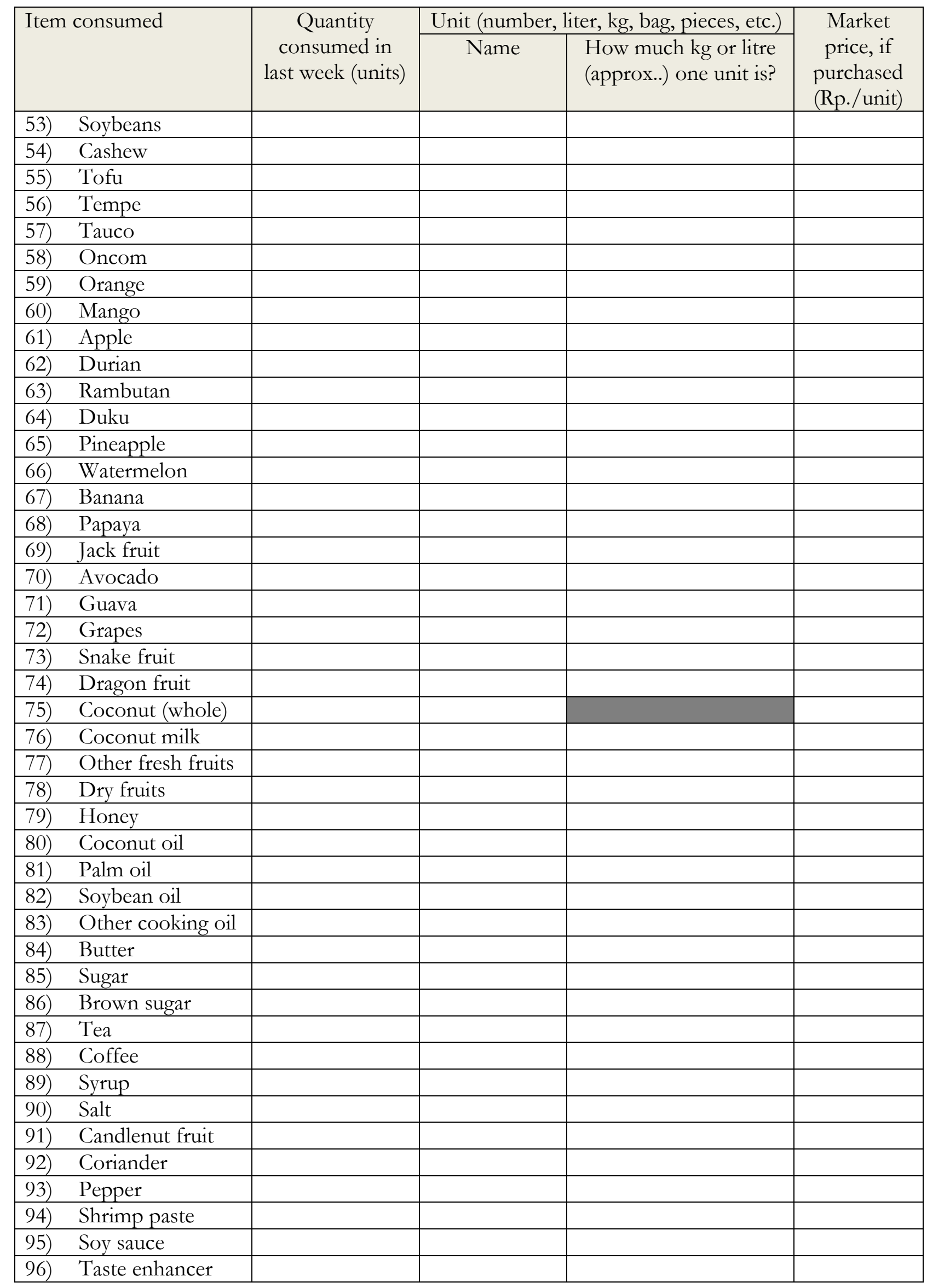




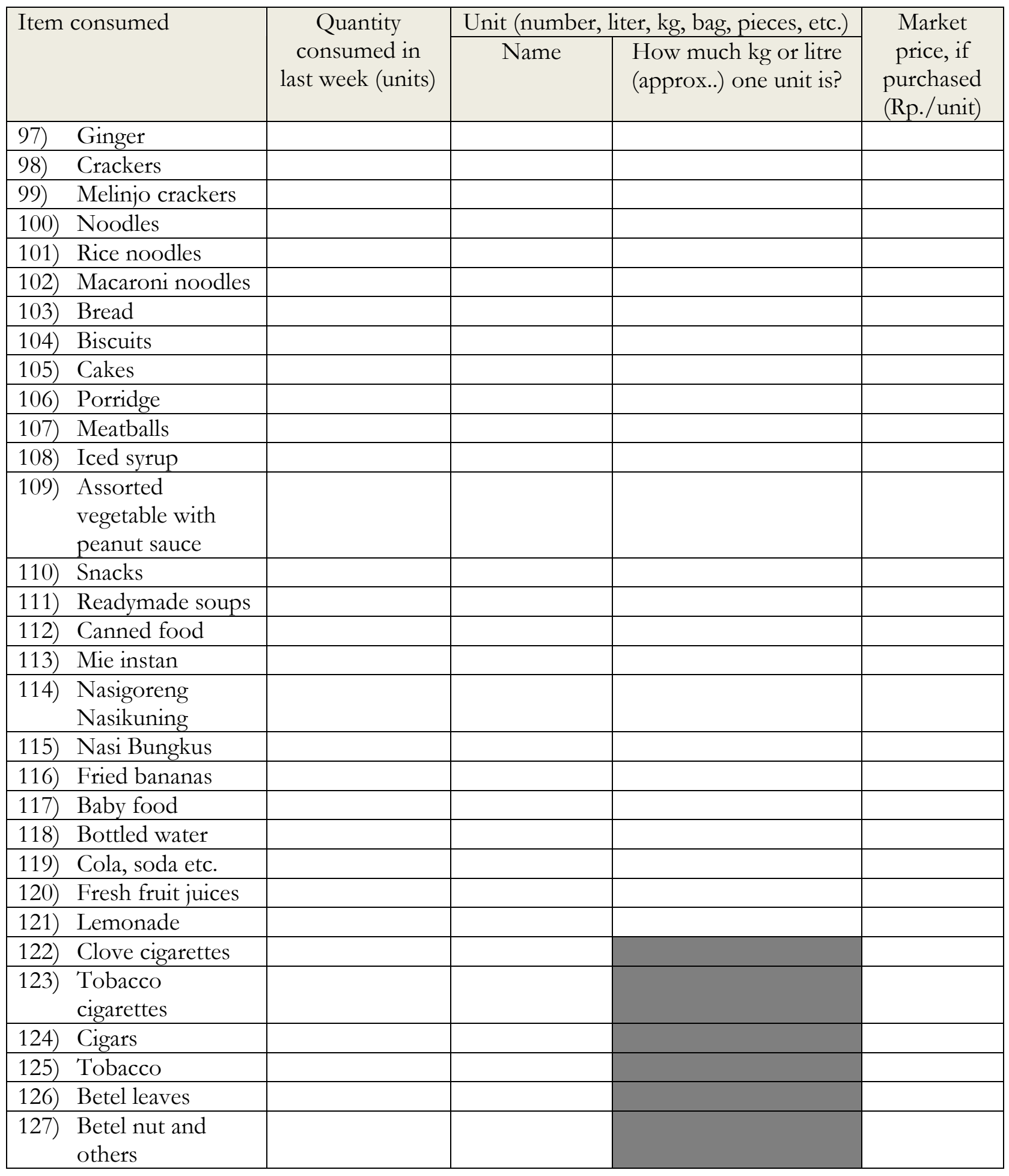




\begin{tabular}{|l|l|l|l|}
\hline Outside house food consumption & $\begin{array}{c}\text { No. of times in } \\
\text { last week }\end{array}$ & No. people/time & $\begin{array}{c}\text { Cost ('000 } \\
\text { Rp/person/time) }\end{array}$ \\
\hline 128) Breakfast & & & \\
\hline 129) Lunch & & & \\
\hline 130) Dinner & & & \\
\hline 131) Tea/Coffee/Snacks & & & \\
\hline
\end{tabular}

2.2. Monthly and annual consumption: Has your household bought or received gifts during the past 30 days/ 12 months? Please exclude from your answer any purchases for processing or resale in a household enterprise.

\begin{tabular}{|c|c|c|c|}
\hline Item & & $\begin{array}{c}\text { Monthly expenditure ('000 } \\
\text { Rp./month) }\end{array}$ & $\begin{array}{c}\text { Yearly expenditure ('000 } \\
\text { Rp. /year) }\end{array}$ \\
\hline 137) & Rent of house if contracted & & \\
\hline 138) & Rent, estimated if house is owned & & \\
\hline 139) & Electricity bill (not for generator) & & \\
\hline 140) & Telephone bill (fixed phone line) & & \\
\hline 141) & Gas bill (kitchen) & & \\
\hline 142) & Kerosene bill & & \\
\hline 143) & Water bill & & \\
\hline 144) & Firewood & & \\
\hline 145) & House maintenance and renovation & & \\
\hline 146) & $\begin{array}{l}\text { Personal care items (soap, shampoo, } \\
\text { toothpaste, etc.) }\end{array}$ & & \\
\hline 147) & $\begin{array}{l}\text { Personal services (haircuts, shaving, } \\
\text { etc.) }\end{array}$ & & \\
\hline 148) & Cosmetics & & \\
\hline 149) & Tailoring expenses & & \\
\hline 150) & Laundry & & \\
\hline 151) & Newspaper and magazines & & \\
\hline 152) & Membership fees & & \\
\hline 153) & Toys & & \\
\hline 154) & Making of ID card/ driver's license & & \\
\hline 155) & Telephone card (mobile phone) & & \\
\hline 156) & Postal goods & & \\
\hline 157) & Recreation & & \\
\hline 158) & Entertainment (e.g., movies, drama) & & \\
\hline 159) & Travel & & \\
\hline 160) & Ornaments & & \\
\hline 161) & Registration fee & & \\
\hline 162) & SPP & & \\
\hline 163) & $\begin{array}{l}\text { POMG/BP3 / entrance- / re- } \\
\text { registration fee }\end{array}$ & & \\
\hline 164) & Boy scout & & \\
\hline 165) & Handcraft & & \\
\hline 166) & Courses & & \\
\hline
\end{tabular}




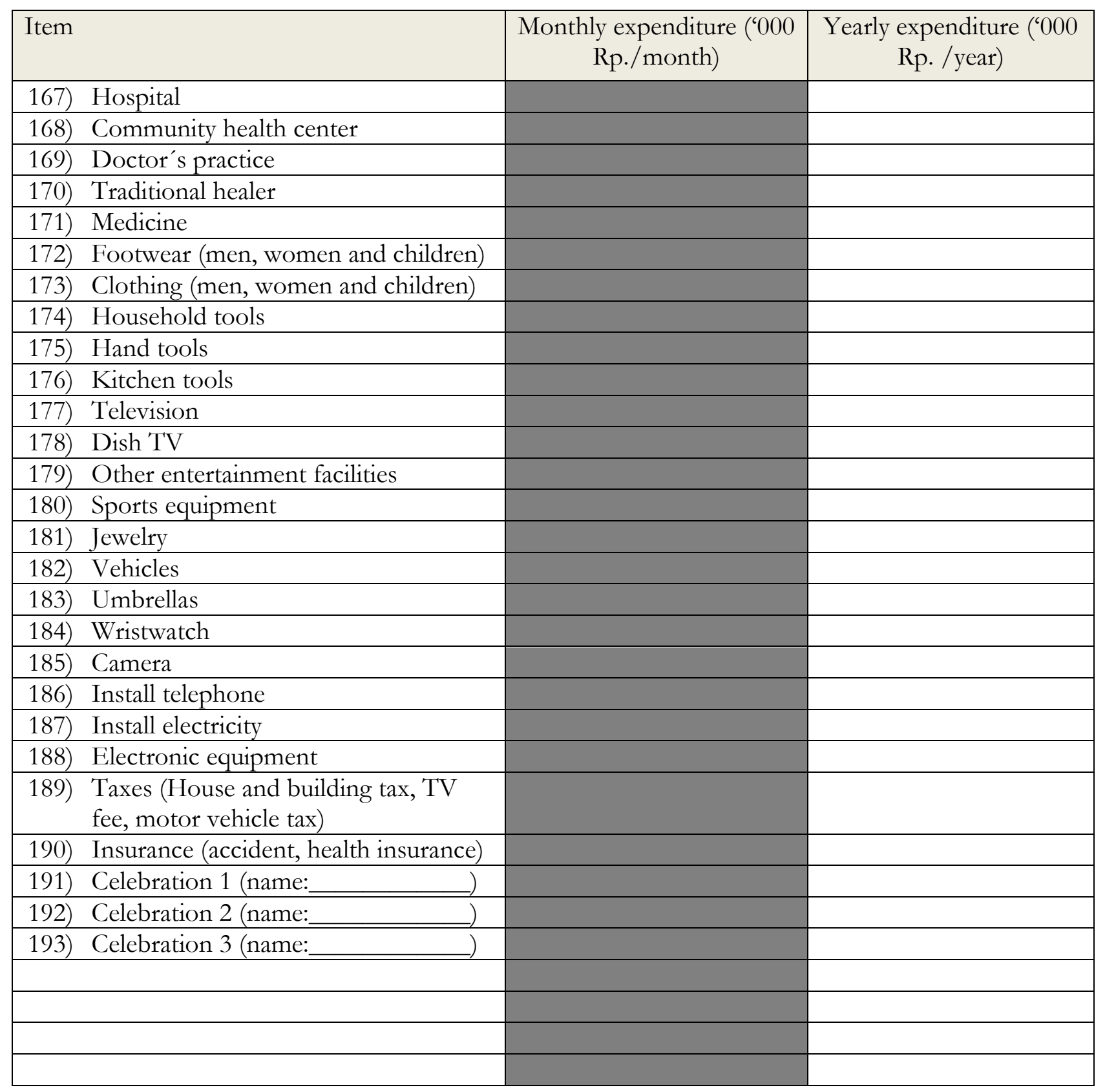


2.3 Consumption of energy (fuel, light \& household appliances) during the last 30 days

\begin{tabular}{|c|c|c|c|c|}
\hline Item & & $\begin{array}{c}\text { Unit } \\
\text { (name) }\end{array}$ & $\begin{array}{l}\text { Quantity consumed in } \\
\text { last one month (units) }\end{array}$ & $\begin{array}{l}\text { Market price ('000 Rp. } \\
\text { / unit) if purchased }\end{array}$ \\
\hline \multicolumn{5}{|c|}{ 194) Dung cake } \\
\hline \multicolumn{5}{|c|}{ 195) Coal, charcoal, briquettes, coke } \\
\hline \multicolumn{5}{|c|}{ 196) LPG [excl. conveyance] } \\
\hline \multicolumn{5}{|c|}{ a. $3 \mathrm{~kg}$ (subsidized) } \\
\hline \multicolumn{5}{|c|}{ b. $15 \mathrm{~kg}$ (non-subsidized) } \\
\hline \multicolumn{5}{|c|}{ 197) Battery } \\
\hline \multicolumn{5}{|c|}{ 198) Accu/ aki (car battery) } \\
\hline \multicolumn{5}{|c|}{ 199) Generator } \\
\hline \multicolumn{5}{|c|}{ a. Petrol (only for generator) } \\
\hline \multicolumn{5}{|c|}{ b. Diesel (only for generator) } \\
\hline \multicolumn{5}{|c|}{$\begin{array}{l}\text { c. Lubricants oil (only for } \\
\text { generator) }\end{array}$} \\
\hline \multicolumn{5}{|c|}{$\begin{array}{l}\text { d. Oil for generator } \\
\text { maintenance (minyak } \\
\text { rem, kanvas, etc.) }\end{array}$} \\
\hline \multicolumn{5}{|c|}{ 200) Other fuel } \\
\hline 201) & $\begin{array}{l}\text { Other consumption (Matches, } \\
\text { candle, air freshener, Mosquito } \\
\text { repellent etc.) }\end{array}$ & & & \\
\hline
\end{tabular}

2.4 Public transport expenditures during the last 30 days

\begin{tabular}{|c|c|c|}
\hline Item & & Total expenditure in last month ('000 Rp) \\
\hline 202) & Public bus/tram fare & \\
\hline 203) & Public minibus (angkot) fare & \\
\hline 204$)$ & Air fare & \\
\hline 205$)$ & Public motorcycle (ojek) & \\
\hline 206$)$ & Taxi, auto-rickshaw fare & \\
\hline 207$)$ & Rental car & \\
\hline & $\begin{array}{l}\text { Other public conveyance expense (such as } \\
\text { porter charges, horse cart fare, etc.) }\end{array}$ & \\
\hline
\end{tabular}

2.5 Private transport expenditures during the last 30 days

\begin{tabular}{|l|l|l|}
\hline Item & $\begin{array}{c}\text { Fuel cost in last } \\
\text { month ('000 Rp) }\end{array}$ & $\begin{array}{c}\text { Other expenditures in last month } \\
\text { (lubricants, other fuel for vehicle, } \\
\text { oil for maintenance, etc.) ('000 } \\
\text { Rp) }\end{array}$ \\
\hline 209$)$ Private car & & \\
\hline 210$)$ Private minibus & & \\
\hline 211$)$ Private bus & & \\
\hline 212$)$ Private motorcycle & & \\
\hline 213$)$ Other private transport & & \\
\hline
\end{tabular}




\subsection{Hadj}

Religion of the household members: $\quad$ Islam/Others (If others, go to 3)

If Islam, have any of the household members gone to Hadj? Yes/No

If yes, details of past pilgrimages:

\begin{tabular}{|c|c|c|}
\hline Year of Hadj & $\begin{array}{c}\text { Number of household } \\
\text { members went }\end{array}$ & $\begin{array}{c}\text { If gone for Hadj after 2012, } \\
\text { expenditure incurred (million Rp) }\end{array}$ \\
\hline & & \\
\hline & & \\
\hline & & \\
\hline
\end{tabular}

Are you planning to go for Hadj in the near future? Yes/No

If yes, are you saving for Hadj, currently? Yes/No

If yes, average savings, kept for this purpose: ............ thousand Rp/year.

\section{Decision-making}

3.1. Who is primarily responsible for the following consumption expenditure items and tasks? (Ask only if the respondent in section 1-3 is different from section 4)

\begin{tabular}{|c|c|c|c|c|}
\hline \multirow[t]{2}{*}{$\begin{array}{c}\text { Consumption and task } \\
\text { items }\end{array}$} & \multirow[t]{2}{*}{ Examples } & \multicolumn{3}{|c|}{$\begin{array}{l}\text { Persons involved (Put } \sqrt{\text { mark or NA if not }} \\
\text { applicable) }\end{array}$} \\
\hline & & $\begin{array}{c}\text { Male } \\
\text { members }\end{array}$ & $\begin{array}{l}\text { Female } \\
\text { members }\end{array}$ & $\begin{array}{l}\text { Both male and } \\
\text { female } \\
\text { members } \\
\text { equally }\end{array}$ \\
\hline Purchase of food items & Rice, vegetables, meat etc. & & & \\
\hline Paying the bills & $\begin{array}{l}\text { Telephone, electricity, gas } \\
\text { etc. }\end{array}$ & & & \\
\hline $\begin{array}{l}\text { Selecting clothing and } \\
\text { footwear }\end{array}$ & $\begin{array}{l}\text { Clothes, tailoring, footwear } \\
\text { etc. }\end{array}$ & & & \\
\hline $\begin{array}{l}\text { Paying for recreation } \\
\text { and membership }\end{array}$ & Movies etc. & & & \\
\hline $\begin{array}{l}\text { Spending on education } \\
\text { of children (if applicable) }\end{array}$ & School fees, books etc. & & & \\
\hline Travel and transport & Taxi, public bus etc. & & & \\
\hline $\begin{array}{l}\text { Purchase and sale of } \\
\text { durable goods }\end{array}$ & Purchasing television etc. & & & \\
\hline $\begin{array}{l}\text { Purchasing and sale of } \\
\text { land and houses }\end{array}$ & $\begin{array}{l}\text { Including involving in } \\
\text { sharecropping }\end{array}$ & & & \\
\hline $\begin{array}{l}\text { Representing the } \\
\text { household in the public }\end{array}$ & $\begin{array}{l}\text { Talking to govt. officials, } \\
\text { participating in the } \\
\text { discussions and group } \\
\text { meetings etc. }\end{array}$ & & & \\
\hline
\end{tabular}




\section{$\underline{\text { Curriculum Vitae }}$}

Name: Christoph KUBITZA

Date/Place of Birth: 22 July 1989; Sandton (South Africa)

Nationality: German

Email: christophkubitza@web.de

Official Address: Department of Agricultural Economics and Rural Development Georg-August-University of Goettingen; Platz der Goettinger Sieben 5; 37073 Goettingen; Germany; Tel. +49 (0) 551 39-22 114; Email: ckubitz@gwdg.de

\section{Current Position}

$04 / 2015-$

Junior Researcher/PhD-student in the Collaborative Research Center 990: Ecological and socioeconomic functions of tropical lowland rainforest transformation systems (Sumatra, Indonesia) at Georg-August University Göttingen

\section{Education}

$04 / 2013-04 / 2015$

$04 / 2011-04 / 2013$

$08 / 2012-01 / 2013$

$10 / 2009-04 / 2011$

\section{Professional Experience}

$08 / 2015-02 / 2016$

$08 / 2014-10 / 2014$

$01 / 2014-12 / 2014$

$10 / 2013-03 / 2014$

$08 / 2013-10 / 2013$

$08 / 2011-10 / 2011$
M. A. in Development Economics at Georg-August University Göttingen

B. A. in International Economics and Development at Bayreuth University

Exchange semester, Economics at Universidade Federal do Rio Grande do Sul in Brazil

Study of Economics and Portuguese Studies at Georg-August University Göttingen

Field Research Supervisor in Indonesia on behalf of the University of Göttingen

Field Research Supervisor in Uganda on behalf of the German Institute for Global and Area Studies (GIGA)

Research assistant at the German Institute for Global and Area Studies (GIGA)

Tutor for Macroeconomics at Georg-August University Göttingen

Internship/Research assistant at the German Institute for Global and Area Studies (GIGA)

Internship in microfinance at NaCSA in Sierra Leone 
Publication Record and Work in Progress (February 2018)

Krishna, V. V.; Kubitza, C.; Pascual, U.; \& Qaim, M. (2017). Land markets, property rights, and deforestation: Insights from Indonesia. World Development 99, 335-349.

Kubitza, C.; Krishna, V. V.; Urban, K.; Zulkifli, A.; \& Qaim, M. (2018). Land property rights, agricultural intensification, and deforestation in Indonesia. Ecological Economics, 147, 312-321.

Kubitza, C.; Krishna, V. V.; Zulkifli, A.; \& Qaim, M. (2018). The Economics behind the Ecological Crisis: Livelihood Effects of Oil Palm Expansion in Sumatra, Indonesia. Human Ecology. https://doi.org/10.1007/s10745-017-9965-7

\section{Conference Presentations}

"Land property rights and agricultural intensification at forest margins in Indonesia" 2017: Annual World Bank Conference on Land and Poverty (Washington, D. C.) 2017: European Association of Agricultural Economists Congress (Parma)

\section{Languages}

German, English, Portuguese, Indonesian 